Louisiana State University

LSU Digital Commons

1992

\title{
The Stability of Thick, Self-Gravitating Disks in Protostellar Systems.
}

John William Woodward

Louisiana State University and Agricultural \& Mechanical College

Follow this and additional works at: https://digitalcommons.Isu.edu/gradschool_disstheses

\section{Recommended Citation}

Woodward, John William, "The Stability of Thick, Self-Gravitating Disks in Protostellar Systems." (1992). LSU Historical Dissertations and Theses. 5365.

https://digitalcommons.Isu.edu/gradschool_disstheses/5365

This Dissertation is brought to you for free and open access by the Graduate School at LSU Digital Commons. It has been accepted for inclusion in LSU Historical Dissertations and Theses by an authorized administrator of LSU Digital Commons. For more information, please contact gradetd@lsu.edu. 


\section{INFORMATION TO USERS}

This manuscript has been reproduced from the microfilm master. UMI films the text directly from the original or copy submitted. Thus, some thesis and dissertation copies are in typewriter face, while others may be from any type of computer printer.

The quality of this reproduction is dependeat upon the quality of the copy submitted. Broken or indistinct print, colored or poor quality illustrations and photographs, print bleedthrough, substandard margins, and improper alignment can adversely affect reproduction.

In the unlikely event that the author did not send UMI a complete manuscript and there are missing pages, these will be noted. Also, if unauthorized copyright material had to be removed, a note will indicate the deletion.

Oversize materials (e.g., maps, drawings, charts) are reproduced by sectioning the original, beginning at the upper left-hand corner and continuing from left to right in equal sections with small overlaps. Each original is also photographed in one exposure and is included in reduced form at the back of the book.

Photographs included in the original manuscript have been reproduced xerographically in this copy. Higher quality $6^{\prime \prime} \times 9^{n}$ black and white photographic prints are available for any photographs or illustrations appearing in this copy for an additional charge. Contact UMI directly to order.

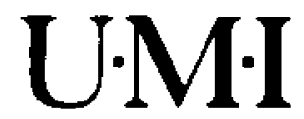

University Microlıims International

A Bell 8 Howell Intormation Company

300 North Zeeb Road. Ann Arbor. MI 48106-1346 USA

$313: 761.4700 \quad 800 \cdot 521.0600$ 
Order Number 9801118

The stability of thick, self-gravitating diske in protostellar aystems

Woodward, John William, Ph.D.

The Louinina State Univernity and Agricultural and Mechanical Col, 1992 


\title{
THE STABILITY OF THICK, SELF-GRAVITATING DISKS IN PROTOSTELLAR SYSTEMS
}

\author{
A Dissertation \\ Submitted to the Graduate Faculty of the \\ Louisiana State University and \\ Agricultural and Mechanical College \\ in partial fulfillment of the \\ requirements for the degree of \\ Doctor of Philosophy \\ in
}

The Department of Physics and Astronomy

by

John William Woodward

B.S., The University of Texas at El Paso, 1986

M.S., Louisiana State University, 1988

May 1992 


\section{ACKNOWLEDGEMENTS}

LSU has certainly become a treasured place in my heart. A great many people have made this stay an enjoyable and productive one.

First and foremost, I must express my gratitude towards my wife, who tolerated the lifestyle of a graduate student for all these years. Without her love and support, none of this would have been possible. In addition, the smiles on my children's faces every time I walk through the door at home has provided a keen sense of perspective on life.

My advisor, Dr. Joel E. Tohline, and his family deserve a great deal of thanks for their support, help, and advice. Most appreciated has been the freedom Joel has given me to let my mind wander through the computing field as much as, if not more than, through the physics field.

A host of others deserve recognition and thanks: Drs. Jerry Draayer and Richard Haymaker, for providing the extra support that meant completion of the doctoral program; Drs. Harold Slusher and Juan Lawson, for teaching me how not to quit; Francesca Verdier and the rest of the staff at Cornell for teaching me parallel computing and tolerating my feeble cries for help; the students I have befriended; my in-laws, for a safe haven in El Paso when things got rough; and of course, my parents, for providing a great beginning to my life, as well as the love and support throughout graduate school.

Finally, I acknowledge financial sponsorship by the NSF through grants AST8701503 and AST-9008166. The extensive computational work required for this study has been conducted primarily at the Cornell National Supercomputer Facility, a resource of the Center for Theory and Simulation in Science and Engineering at 
Cornell University, which is funded in part by the National Science Foundation, New York State, and the IBM Corporation. 


\section{TABLE OF CONTENTS}

Acknowledgements . . . . . . . . . . . . . . ii

List of Tables . . . . . . . . . . . . . . . . . . . . . vi

List of Figures . . . . . . . . . . . . . . . . . . . . . . . . . . . . vii

Abstract . . . . . . . . . . . . . . . . xiii

1 Introduction ...................... 1

1.1 Observational Constraints . . . . . . . . . . . . . . . . . . . 2

1.2 Basic Theoretical Scenario . . . . . . . . . . . . . . . . . . 6

1.3 Relationship to Previous Studies of Disk Stability . . . . . . . . . 8

2 Isolated, Self-Gravitating Disks . . . . . . . . . . . . . . . . . . . 12

2.1 Introduction . . . . . . . . . . . . . . . . . . . . . . . . . . 12

2.2 Hydrodynamic Code Comparison . . . . . . . . . . . . . . . . . . . 17

2.3 Analysis of the Data . . . . . . . . . . . . . . . . . . . . . . 19

2.4 An Idealized Model . . . . . . . . . . . . . . . . . . . . . . . 22

2.5 Selected Massive Models . . . . . . . . . . . . . . . . . . . . 27

2.6 Results and Comparison . . . . . . . . . . . . . . . . . . . . 28

3 Self-Gravitating Disks in the Presence of an Inert,

Central Point Mass . . . . . . . . . . . . . . . . . . . . . . 49

3.1 Introduction . . . . . . . . . . . . . . . . . . . . . . . . . . 49

3.2 Heavy Disks: Straightforward Evolutions . . . . . . . . . . . . . 49

3.2.1 Systems with $M_{d} / M_{c}=5.0$. . . . . . . . . . . . . . . . 51

3.2.2 Systems with $M_{d} / M_{c}=1.0$. . . . . . . . . . . . . . . . .68

3.2.3 Systems with $M_{d} / M_{c}=0.2$. . . . . . . . . . . . . . . . . 85

3.3 Summary of Even-mode Evolutions . . . . . . . . . . . . . . . 107

4 Response of the Central Object to a Massive, Axisymmetric Disk

(The Axisymmetric Roche Problem) . . . . . . . . . . . . . . 111

4.1 Introduction . . . . . . . . . . . . . . . . . . . . . . . . . 111

4.2 The Combined Disk, Central-Object Potential . . . . . . . . . . 111

4.2.1 Analytic Model . . . . . . . . . . . . . . . . . 111

4.2.2 Self-Consistent-Field Models of Thick Disks . . . . . . . . . 115

4.3 The Condition for Runaway Excretion . . . . . . . . . . . . . 118

4.4 Discussion . . . . . . . . . . . . . . . . . . . . . 122

5 Disk Instabilities Resulting from Interactions with the Centra]

Point Mass . . . . . . . . . . . . . . . . . . . . . . . . . 128 
5.1 Introduction . . . . . . . . . . . . . . . . . . . . . 128

5.2 Resonant Mass Ratio - Crude Calculation . . . . . . . . . . . 129

5.3 Linear Theory Predictions . . . . . . . . . . . . . . . . . . . 132

5.4 Computational Considerations . . . . . . . . . . . . . . . . . 133

5.4.1 Systems with $M_{d} / M_{c}=5.0$. . . . . . . . . . . . . . . . 135

5.4.2 Systems with $M_{d} / M_{c}=1.0$. . . . . . . . . . . . . . . . 153

5.4.3 Systems with $M_{d} / M_{c}=0.2$. . . . . . . . . . . . . . . 170

5.5 Summary of Odd-mode Evolutions . . . . . . . . . . . . . . . 182

6 Conclusions . . . . . . . . . . . . . . . . . . . . 186

6.1 Summary of Simulations _. . . . . . . . . . . . . . . . . . 186

6.2 The Relationship to Star Formation . . . . . . . . . . . . . . 189

References . . . . . . . . . . . . . . . . . . . . . 194

Appendix A: Details Concerning the Hydrodynamic Code . . . . . . . 197

A.1 Introduction . . . . . . . . . . . . . . . . . . . . . . . . 197

A.2 Sourcing . . . . . . . . . . . . . . . . . . . . . . . . . . 198

A.3 van Leer Monotonic Interpolation . . . . . . . . . . . . . . . 199

Appendix B: Determination of $y_{1}$ and $y_{2} \ldots \ldots$. . . . . . . . . . . 203

Appendix C: Conversion to Physical Units . . . . . . . . . . . . . . 209

Appendix D: A Direct Comparison with Linear Theory . . . . . . . . 212

Vita . . . . . . . . . . . . . . . . . . . . . . . . . 219 


\section{LIST OF TABLES}

Table 2.1: Initial Model Data for $M_{d} / M_{c}=\infty$. . . . . . . . . . . 32

Table 2.2: $\mathrm{m}=2$ Eigenmode Character, $M_{d} / M_{\mathrm{c}}=\infty$. . . . . . . . . . . 33

Table 3.1: Initial Model Data for $M_{d} / M_{\mathrm{c}}=5.0$. . . . . . . . . . 53

Table 3.2: $\mathrm{m}=2$ Eigenmode Character, $M_{d} / M_{c}=5.0 \quad \ldots . . . . .54$

Table 3.3: Initial Model Data for $M_{d} / M_{c}=1.0 \ldots \ldots$

Table 3.4: $\mathrm{m}=2$ Eigenmode Character, $M_{d} / M_{c}=1.0 \quad \ldots \ldots . . . . .71$

Table 3.5: Initial Model Data for $M_{d} / M_{c}=0.2 \ldots . . . . . . . .90$

Table 3.6: $\mathrm{m}=2$ Eigenmode Character, $M_{d} / M_{c}=0.2 \quad \ldots . . . . . . .91$

Table 4.1: Location of the Potential Maximum . . . . . . . . . . . . 114

Table 4.2: Special Points in Figure 4.3 . . . . . . . . . . . . . . . . 121

Table 5.1: Initial Model Data for $M_{d} / M_{c}=5.0 \ldots \ldots$

Table 5.2: $m=1$ Eigenmode Character, $M_{d} / M_{c}=5.0 \quad \ldots \ldots . . . . .140$

Table 5.3: Initial Model Data for $M_{d} / M_{c}=1.0 \ldots \ldots 5$

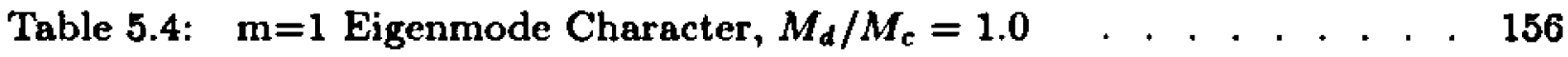

Table 5.5: Initial Model Data for $M_{d} / M_{c}=0.2$. . . . . . . . . . . . 171

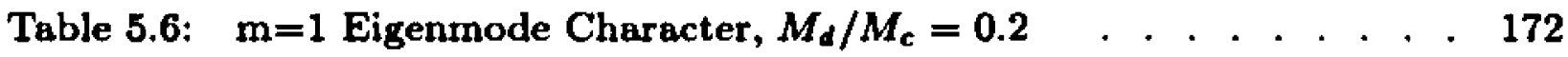

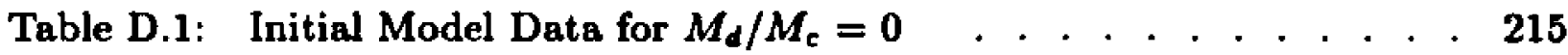

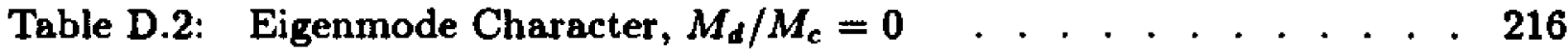




\section{LIST OF FIGURES}

Figure 1.1: Specific angular momentum of binary star systems . . . . . . 5

Figure 2.1: Meridional cross-section through an exanple model . . . . . 14

Figure 2.2: $T /|W|$ vs mass ratio template . . . . . . . . . . . . . . 16

Figure 2.3a: $m=2$ amplitude and phase for an idealized model . . . . . . 23

Figure 2.3b: $\quad m=2$ phase vs. radius and phase vs. amplitude for an idealized model . . . . . . . . . . . . . . . . . . . . 25

Figure 2.4a: $m=2$ amplitude and phase for $M_{d} / M_{c}=\infty$, model \#1 . . 34

Figure 2.4b: $m=2$ phase vs. radius and phase vs. amplitude for $M_{d} / M_{\mathrm{c}}=\infty$, model \#1 . . . . . . . . . . . . . . 35

Figure 2.5a: $m=2$ amplitude and phase for $M_{d} / M_{c}=\infty$, model \#2 . . 36

Figure 2.5b: $m=2$ phase vs. radius and phase vs. amplitude for $M_{d} / M_{c}=\infty$, model \#2 . . . . . . . . . . 37

Figure 2.6a: $\quad m=2$ amplitude and phase for $M_{d} / M_{c}=\infty$, model \#4 . . 38

Figure 2.6b: $m=2$ phase vs. radius and phase vs. amplitude for $M_{d} / M_{c}=\infty$, model \#4 . . . . . . . . . . . . . . . . . 39

Figure 2.7a: $m=2$ amplitude and phase for $M_{d} / M_{c}=\infty$, model \#5 . . 40

Figure 2.7b: $m=2$ phase vs. radius and phase vs. amplitude for $M_{d} / M_{c}=\infty$, model \#5 ............ . 41

Figure 2.8a: $\quad m=2$ amplitude and phase for $M_{d} / M_{c}=\infty$, model \#6 . . . 42

Figure 2.8b: $m=2$ phase vs. radius and phase vs. amplitude for $M_{d} / M_{c}=\infty$, model \#6 . . . . . . . . . . . . . . 43

Figure 2.9a: $\quad m=2$ amplitude and phase for $M_{d} / M_{c}=\infty$, model \#7 . . . 44

Figure 2.9b: $m=2$ phase vs. radius and phase vs. amplitude for $M_{d} / M_{c}=\infty$, model \#7

Figure 2.10a: $m=2$ amplitude and phase for $M_{d} / M_{c}=\infty$, model \#8 $\ldots 46$ 
Figure 2.10b: $m=2$ phase vs. radius and phase vs. amplitude for $M_{d} / M_{c}=\infty$, model $\# 8$

Figure 2.11: $y_{2}(2)$ and $y_{1}(2)$ parameters for mass ratio $\infty$ models . . . . . 48

Figure 3.1: $\quad y_{2}(2)$ and $y_{1}(2)$ parameters for mass ratio 5.0 models... .55

Figure 3.2a: $\quad m=2$ amplitude and phase for $M_{d} / M_{c}=5.0$, model \#1 . . . 56

Figure 3.2b: $m=2$ phase vs. radius and phase vs. amplitude for $M_{d} / M_{c}=5.0$, model \#1

Figure 3.3a: $m=2$ amplitude and phase for $M_{d} / M_{c}=5.0$, model \#2 . . . 58

Figure 3.3b: $m=2$ phase vs. radius and phase vs. amplitude for $M_{d} / M_{c}=5.0$, model $\# 2$

Figure 3.4a: $m=2$ amplitude and phase for $M_{d} / M_{c}=5.0$, model \#3 . . . 60

Figure 3.4b: $m=2$ phase vs. radius and phase vs. amplitude for $M_{d} / M_{c}=5.0$, model $\# 3$

Figure 3.5a: $m=2$ amplitude and phase for $M_{d} / M_{c}=5.0$, model \#4 . . . 62

Figure 3.5b: $m=2$ phase vs. radius and phase vs. amplitude for $M_{d} / M_{c}=5.0$, model \#4

Figure 3.6e: $m=2$ amplitude and phase for $M_{d} / M_{\mathrm{c}}=5.0$, model \#5. .64

Figure 3.6b: $m=2$ phase vs. radius and phase vs. amplitude for $M_{\mathrm{d}} / M_{c}=5.0$, model \#5

Figure 3.7a: $\quad m=2$ amplitude and phase for $M_{d} / M_{c}=5.0$, model \#6 . . . 66

Figure 3.7b: $\quad m=2$ phase vs. radius and phase vs. amplitude for $M_{d} / M_{c}=5.0$, model \#6 67

Figure 3.8: $y_{2}(2)$ and $y_{1}(2)$ parameters for mass ratio 1.0 models $\quad . \quad . \quad .72$

Figure 3.9a: $m=2$ amplitude and phase for $M_{d} / M_{c}=1.0$, model \#1 . . 73

Figure 3.9b: $m=2$ phase vs. radius and phase v8. amplitude for $M_{d} / M_{c}=1.0$, model $\# 1$. . . . . . . . . . . . . . . . . 74

Figure 3.10a: $m=2$ amplitude and phase for $M_{d} / M_{c}=1.0$, model \#2 $\quad$ - . 75 
Figure 3.10b: $m=2$ phase vs. radius and phase vs. amplitude for $M_{d} / M_{c}=1.0$, model \#2

Figure 3.11a: $m=2$ amplitude and phase for $M_{d} / M_{c}=1.0$, model \#3 . . . 77

Figure 3.11b: $m=2$ phase vs. radius and phase vs. amplitude for $M_{d} / M_{c}=1.0$, model \#3

Figure 3.12a: $m=2$ amplitude and phase for $M_{d} / M_{c}=1.0$, model $\# 4 \quad . \quad .79$

Figure 3.12b: $m=2$ phase vs. radius and phase vs. amplitude for $M_{d} / M_{c}=1.0$, model $\# 4$

Figure 3.13a: $m=2$ amplitude and phase for $M_{d} / M_{c}=1.0$, model \#5 . . . 81

Figure 3.13b: $m=2$ phase vs. radius and phase vs. amplitude for $M_{d} / M_{c}=1.0$, model \#5

Figure 3.14a: $m=2$ amplitude and phase for $M_{d} / M_{c}=1.0$, model \#6 . . . 83

Figure 3.14b: $m=2$ phase vs. radius and phase vs. amplitude for $M_{d} / M_{c}=1.0$, model \#6

Figure 3.15: $y_{2}(2)$ and $y_{1}(2)$ parameters for mass ratio 0.2 models $\quad . \quad . \quad$. . 92

Figure 3.16a: $m=2$ amplitude and phase for $M_{d} / M_{c}=0.2$, model \#1 . . . 93

Figure 3.16b: $m=2$ phase vs. radius and phase vs. amplitude for $M_{d} / M_{c}=0.2$, model \#1

Figure 3.17a: $m=2$ amplitude and phase for $M_{d} / M_{c}=0.2$, model \#2 . . . 95

Figure 3.17b: $m=2$ phase vs. radius and phase vs. amplitude for $M_{d} / M_{c}=0.2$, model \#2

Figure 3.18a: $m=2$ amplitude and phase for $M_{d} / M_{c}=0.2$, model \#3 . . . 97

Figure 3.18b: $m=2$ phase vs. radius and phase vs. amplitude for $M_{d} / M_{c}=0.2$, model $\# 3$. . . . . . . . . . . . . . . . . 98

Figure 3.19a: $m=2$ amplitude and phase for $M_{d} / M_{c}=0.2$, model \#4 . . . 99

Figure 3.19b: $m=2$ phase v8. radius and phase vs. amplitude for $M_{\alpha} / M_{c}=0.2$, model \#4 . . . . . . . . . . . . . . . . 100

Figure 3.20a: $m=2$ amplitude and phase for $M_{d} / M_{c}=0.2$, model \#5 . . 101 
Figure 3.20b: $m=2$ phase vs. radius and phase vs. amplitude for $M_{d} / M_{c}=0.2$, model \#5 . . . . . . . . . . . 102

Figure 3.21a: $m=2$ amplitude and phase for $M_{d} / M_{c}=0.2$, model \#6 . . 103

Figure 3.21b: $m=2$ phase vs. radius and phase vs. amplitude for $M_{d} / M_{c}=0.2$, model \#6 . . . . . . . . . . . . . . 104

Figure 3.22a: $m=2$ amplitude and phase for $M_{d} / M_{c}=0.2$, model \#7 , 105

Figure 3.22b: $m=2$ phase vs. radius and phase vs. amplitude for $M_{d} / M_{c}=0.2$, model \#7 . . . . . . . . . . . . . 106

Figure 3.23: $y_{2}(2)$ and $y_{1}(2)$ parameters for $\pi$-symmetry models $\ldots . . . \quad 109$

Figure 3.24: Growth rates of $\pi$-symmetry models . . . . . . . . . . . 110

Figure 4.1: Analytical vo. SCF comparison . . . . . . . . . . . . . 115

Figure 4.2: Potential comparison . . . . . . . . . . . . . . . . . . 116

Figure 4.3: Stability indication . . . . . . . . . . . . . . . . . . 120

Figure 4.4: Dynamical stability velocity criterion . . . . . . . . . . . 125

Figure 4.5: Dynamical stability temperature criterion . . . . . . . . . 126

Figure 5.1: $\quad y_{2}(1)$ and $y_{1}(1)$ parameters for mass ratio 5.0 models . . . . 141

Figure 5.2a: $\quad m=1$ amplitude and phase for $M_{d} / M_{c}=5.0$, model \#1 . 142

Figure 5.2b: $m=1$ phase vs. radius and phase vs. amplitude for $M_{d} / M_{c}=5.0$, model \#1 . . . . . . . . . . 143

Figure 5.2c: Radial behavior of the central object for $M_{d} / M_{c}=5.0$, model \#1. . . . . . . . . . . . . . . . . . . . 144

Figure 5.3a: $m=1$ amplitude and phase for $M_{d} / M_{c}=5.0$, model \#2 . . 145

Figure 5.3b: $m=1$ phase vs. radius and phase vs. amplitude for $M_{\mathrm{d}} / M_{\mathrm{c}}=5.0$, model \#2 . . . . . . . . . . . . . 146

Figure 5.4a: $m=1$ amplitude and phase for $M_{d} / M_{c}=5.0$, model \#3 $\quad$. 147

Figure 5.4b: $m=1$ phase vs. radius and phase vs. amplitude for $M_{d} / M_{c}=5.0$, model \#3 
Figure 5.5a: $\quad m=1$ amplitude and phase for $M_{d} / M_{c}=5.0$, model \#4 $\quad . \quad 149$

Figure 5.5b: $m=1$ phase vs. radius and phase vs. amplitude for $M_{d} / M_{c}=5.0$, model $\# 4$. . . . . . . . . . . . . . 150

Figure 5.6a: $m=1$ amplitude and phase for $M_{d} / M_{c}=5.0$, model \#5 . 151

Figure 5.6b: $m=1$ phase vs, radius and phase vs. amplitude for $M_{d} / M_{c}=5.0$, model $\# 5$. . . . . . . . . . . . . 152

Figure 5.7: $\quad y_{2}(1)$ and $y_{1}(1)$ parameters for mass ratio 1.0 models . . . 157

Figure 5.8a: $\quad m=1$ amplitude and phase for $M_{d} / M_{c}=1.0$, model \#1 . 158

Figure 5.8b: $m=1$ phase vs. radius and phase vs. amplitude for $M_{d} / M_{c}=1.0$, model \#1 . . . . . . . . . . . . . . 159

Figure 5.9a: $\quad m=1$ amplitude and phase for $M_{d} / M_{c}=1.0$, model \#2 . . 160

Figure 5.9b: $m=1$ phase vs. radius and phase vs. amplitude for $M_{d} / M_{c}=1.0$, model \#2 . . . . . . . . . . . . 161

Figure 5.10a: $m=1$ amplitude and phase for $M_{d} / M_{c}=1.0$, model \#3 . . 162

Figure 5.10b: $m=1$ phase vs. radius and phase vs. amplitude for $M_{d} / M_{c}=1.0$, model \#3 . . . . . . . . . . . . 163

Figure 5.11a: $m=1$ amplitude and phase for $M_{d} / M_{c}=1.0$, model \#4 . . 164

Figure 5.11b: $m=1$ phase vs. radius and phase vs. amplitude for $M_{d} / M_{c}=1.0$, model \#4 ............. 165

Figure 5.12a: $m=1$ amplitude and phase for $M_{d} / M_{c}=1.0$, model \#5 . . 166

Figure 5.12b: $m=1$ phase vs. radius and phase vs. amplitude for $M_{d} / M_{c}=1.0$, model $\# 5$. . . . . . . . . . . . . . . 167

Figure 5.13a: $m=1$ amplitude and phase for $M_{d} / M_{c}=1.0$, model \#6 $\quad . \quad 168$

Figure 5.13b: $m=1$ phase vs. radius and phase vs. amplitude for $M_{d} / M_{c}=1.0$, model \#6 . . . . . . . . . . . . . . . . 169

Figure 5.14: $y_{2}(1)$ and $y_{1}(1)$ parameters for mass ratio 0.2 models $\ldots .$.

Figure 5.15a: $m=1$ amplitude and phase for $M_{d} / M_{c}=0.2$, model \#1 . . 174 
Figure 5.15b: $m=1$ phase vs. radius and phase vs. amplitude for $M_{d} / M_{c}=0.2$, model \#1 . . . . . . . . . . 175

Figure 5.16a: $m=1$ amplitude and phase for $M_{d} / M_{c}=0.2$, model \#2 . 176

Figure 5.16b: $m=1$ phase vs. radius and phase vs. amplitude for $M_{d} / M_{c}=0.2$, model \#2 . . . . . . . . . . . . . . . 177

Figure 5.17a: $m=1$ amplitude and phase for $M_{d} / M_{c}=0.2$, model \#3 $\quad$. 178

Figure 5.17b: $m=1$ phase vs, radius and phase vs. amplitude for $M_{d} / M_{c}=0.2$, model \#3 . . . . . . . . . . . . . . 179

Figure 5.18a: $m=1$ amplitude and phase for $M_{d} / M_{c}=0.2$, model \#4 , , 180

Figure 5.18b: $m=1$ phase vs. radius and phase vs. amplitude for $M_{d} / M_{c}=0.2$, model \#4 . . . . . . . . . . . . . 181

Figure 5.19: $y_{2}(2)$ and $y_{1}(2)$ parameters for non- $\pi$-8ymmetry models $\quad$. 184

Figure 5.20: Growth rates of non- $\pi$-symmetry models . . . . . . . . . 185

Figure 6.1: Growth rates of all models evolved . . . . . . . . . . . . 187

Figure 6.2: Hypothetical path to star formation _ . . . . . . . . . . 191

Figure D.1: $\quad y_{2}(m)$ and $y_{1}(m)$ for $M_{d} / M_{c}=0$ models . . . . . . . . 217

Figure D.2: $\quad m=1$ phase vs. radius and phase vs. amplitude for a typical $M_{d} / M_{c}=0$ model . . . . . . . . . . . . . . . 218 


\section{ABSTRACT}

We perform a computational survey of the stability of protostellar systems which contain a self-gravitating disk. The systems are initially represented by a point mass $M_{c}$ at the center and a geometrically thick, axisymmetric disk of mass $M_{d}$ that supports uniform specific angular momentum and obeys an $n=3 / 2$, polytropic equation of state. The equilibrium disk structure is uniquely defined upon the specification of two key dimensionless system parameters: $M_{d} / M_{c}$ and $T /|W|$ (the ratio of rotational kinetic energy of the disk to the gravitational potential energy of the system). The focus of this work is the identification of systems within this two-dimensional parameter space that are marginally unstable toward the development of nonaxisymmetric distortions. The geometric form of the disk's distortion and the likelihood of disk fragmentation as a result of such instabilities is examined with particular attention given to the formation of binary systems.

The principal conclusions of this work are: (a) A computer code which results in data that does not require the application of numerical corrections is essential for the identification of marginally unstable models. (b) Models in which the central object is constrained to remain at the center of mass of the system show two principal instabilities, one supplanting the other as the stability of cooler systems is explored. (c) Models in which the central object is allowed to move and interact dynamically with the disk indicate that two new instabilities emerge. These new instabilities arise in disks that appear to be stable when the central object is constrained not to move. The instability occurring in the marginally unstable systems promotes the development of a tightly wound, one-armed spiral perturbation in the disk. (d) Disk fragmentation via the one-armed spiral mode is consistent with observations indicating that binary formation is the principal branch of the stellar formation process. 


\section{CHAPTER 1 \\ INTRODUCTION}

The formation of multiple star systems is a process that is not understood in any concrete detail. Interstellar molecular clouds, with radii on the order of 0.5 to 1.0 parsecs and masses on the order of $10^{3}$ solar masses $\left(M_{\odot}\right)$, develop regions of enhanced density (so-called, "cloud cores") which can be detected/identified at infrared and millimeter wavelengths through the intervening, relatively low density regions of the clouds. However, the cloud cores - with radii of 0.1 to 0.5 parsecs and masses of a few $M_{\odot}-$ are optically thick, even at millimeter wavelengths, which precludes observations of the dynamics of their interior. It is generally believed, based upon a variety of theoretical arguments and numerical simulations of cloud collapse, that a disk structure forms within the cloud core, with, perhaps, a central protostellar mass forming as well. The dynamics of the disk involved must redistribute angular momentum throughout a collapsing cloud core (see further discussion below), with the end result being the condensation of a single young stellar object, or a multiple stellar system. As discussed in subsequent paragraphs, the frequency of the occurrence of each type of system - single star, binary, triple, etc. - as well as some general characteristics of these systems are subject to certain known constraints, but the actual physical processes involved in establishing these observed frequencies and characteristics are, at this point in time, mostly conjecture. It is the aim of this dissertation to examine the stability of self-gravitating disk structures in the context of star formation in order to better understand the process by which angular momentum redistribution takes place and/or multiple stars form in the dense cores of interstellar molecular clouds. 


\subsection{Observational Constrainte}

One boundary condition on the properties that the ultimate results of the collapse of a molecular cloud core must satisfy is provided by observations of star systems containing multiple components in the main sequence (MS) stage of their evolution. Although MS stars are far removed in time from the initial stages of stellar formation and, as such, do not reveal the actual physical processes that took place at the time of their formation, the ultimate outcome can be observed. These properties are ascertained through extensive surveys of MS stars with masses comparable to our own sun. The principal surveys that we will draw on for the present discussion are the classic study of Abt and Levy (1976, hereafter, AL; see also, Abt 1987), and Duquennoy and Mayor (1991; DM). Bodenheimer, Ruzmaikina, and Mathieu (1991; BRM) have reviewed the properties of MS binary systems in some detail; for this discussion, it is enough to present some general properties without such attention to detail.

1. Half of all stars included in these surveys are directly detected to be members of multiple systems. When this figure is corrected to account for selection biases, the actual frequency of multiple systems is thought to be as high as $78 \%$ (Abt 1983). Of these multiple systems, $85 \%$ are detected specifically as binary systems.

2. The orbital period distribution of binary systems is continuous, with a single maximum, covering 8 orders of magnitude in the range $-3<\log$ Period(years) $<7$. The median period is 180 years; the percentage of systems having $P<100$ days is $12 \%$.

3. The mass-ratio distribution function is not as clearly determined as the prior two properties. For longer-period binaries, the secondary mass distribution 
rises continuously toward smaller mass. The case for shorter-period binaries has received particular attention due to its uncertainty; if the distribution differs from the longer-period binaries it may indicate differing formation processes. $A L$ and Abt (1987) argue that the primary to secondary mass ratio tends to be closer to unity for shorter-period binaries, with a transition period of $\approx 100 \mathrm{yrs}$. DM, however, observe no such transition from longer-period to shorter-period binaries in the secondary mass function. This is an observational point that needs to be clarified in the future.

4. The eccentricity of binary orbits is very clearly period dependent. For MS binary systems, orbits are circular for periods less than $10-13$ days; systems with periods greater than this transition period have eccentric orbits, with essentially all values of the eccentricity present in the sample data. This transition period appears to be age dependent in the sense that the older the system, the longer the transition period. This is entirely consistent with the idea that efficient circularization of binary orbits can be achieved through tidal interactions between the components of the system.

Observations of low-mass (primary $\approx 1 M_{\odot}$ ) pre-main sequence (PMS) binary systems provide a point in the evolution of such systems much closer to the time of the formation of the individual components. Unfortunately, extensive surveys of PMS systems are only now being carried out; hence, the data available is sparse. BRM list data for the 11 spectroscopic binaries whose characteristics had been published at the time of the submission of their article, stating that the current number of known spectroscopic binaries is greater than 20. While other observational techniques are also used in the observation of PMS binaries, the spectroscopic surveys provide the richest data corresponding to the properties enumerated above. Thus, for the most part, conclusions drawn from other observational techniques will not be 
discussed here. A listing of properties corresponding to those given for MS binary surveys is detailed below.

1. The frequency of PMS binaries with periods less than 100 days is comparable to the corresponding binary frequency among MS stars ( $9 \% \pm 4 \%$ v8. $12 \%)$.

2. Binaries of virtually all periods have been observed, regardless of the age of the system (note, however, long period binaries are sparse in the data due to the recent date at which PMS binary surveys began). Of particular note are DF Tau and SVS 20, two PMS binary systems whose ages have been estimated to be $\sim 10^{5}$ years. These two systems alone indicate that binary formation can occur very early in the star formation process.

3. A determination of the mass-ratio distribution function for so few objects cannot be made, let alone a comparison between MS and PMS distribution functions. In any case, the longest period spectroscopic binary listed by BRM is less than 1 year, much less than the transition period of $\approx 100$ years for MS binaries observed by AL. What can be determined from mass determinations is that, in general, binary companions form coevally to within an accuracy of $10^{6}$ years.

4. Low-mass spectroscopic PMS binaries show the same period dependence in orbital eccentricity as MS binaries, with a transition period slightly greater than 4 days. Given the young age of these systems, this shorter transition period would be expected if the circularization of binary orbits is due to tidal interactions.

The specific angular momentum, $j$, of binary star systems (assuming a perfectly circular orbit) is given by the following expression:

$$
j=4.45 \times 10^{19} \frac{M_{1} M_{2}}{\left(M_{1}+M_{2}\right)^{4 / 3}} P^{1 / 3} \mathrm{~cm}^{2} \mathrm{sec}^{-1},
$$




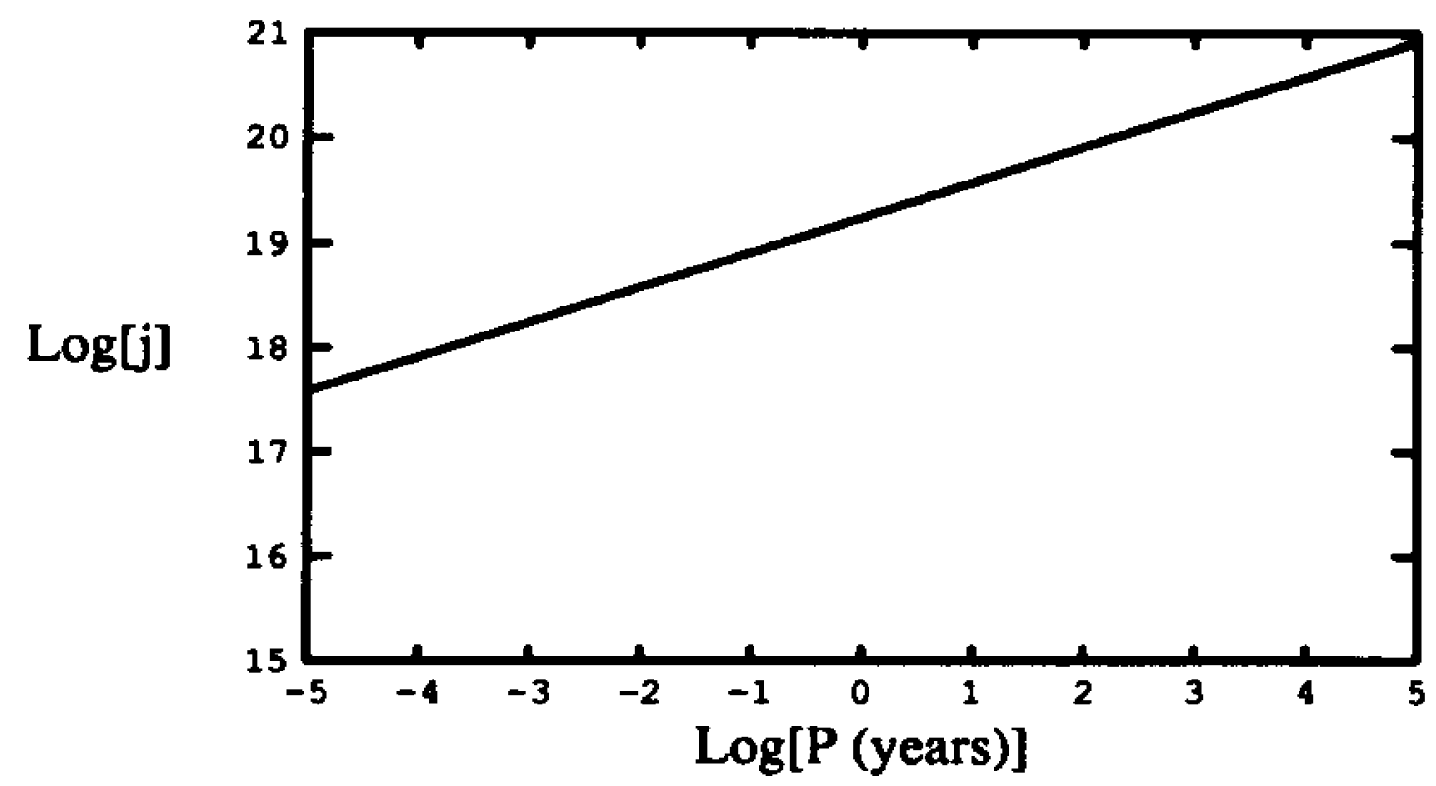

Figure 1.1: The logarithm of the specific angular momentum $j$ of a binary system consisting of two solar mass components is plotted versus the logarithm of the period to illustrate relation (1.1).

where the masses $M_{1}$ and $M_{2}$ are given in solar masses, and the period is given in years (this relation is illustrated in Figure 1.1). Hence, even the longest period binary systems $\left(P \sim 10^{3}\right.$ years $)$ possess values of $j$ below the most accurate measurements to date of the specific angular momentum of molecular clouds $\left(j \approx 10^{23}\right)$. Cloud cores themselves are observed to have a $j$ value at least two orders of magnitude below that of the molecular clouds as a whole $\left(j \lesssim 10^{21}\right)$, comparable to that of long-period binaries. Short period binaries $\left(j \approx 10^{18}\right)$ have $j$ 's 2-3 orders of magnitude below the long period binaries, whereas single solar type stars have spin $j$ values some 3 orders of magnitude below the short period binaries $\left(j \approx 10^{15}\right)$. Clearly, at some point during the star formation process, the angular momentum present in the initial cloud is redistributed away from the collapsing cloud cores, 
and the angular momentum remaining in the cloud core is, in turn, redistributed as the collapse proceeds towards stellar formation.

Any acceptable theory of the star formation process must explain in general terms why stars preferentially form in multiple systems and, more specifically, must explain why multiple and single stellar systems form with the properties described above. At this point in time, there is no single theory which does so; the best that can be done is to describe events that may occur as a molecular cloud gives birth to stars. These events derive principally through basic theoretical arguments, coupled with hydrodynamic calculations of the collapse.

\subsection{Basic Theoretical Scenario}

Magnetic fields can have an appreciable effect on the dynamical behavior of the relatively low density regions of molecular clouds due to ionization of the material in these regions. Whether condensing from the molecular cloud due to a gravitational Jeans' instability (eg., Lizano and Shu 1989) or from a turbulent inhomogeneity within the cloud (eg., Tohline, Bodenheimer, \& Christodoulou 1987), cloud cores are not thought to be aignificantly ionized and, hence, are not subject to these magnetic effects. The result is that the magnetic field may act to effectively slow the rotation of the cloud core as it begins to condense from the low density molecular cloud, but magnetic braking is believed not to be important after the core reaches a density at which the field decouples from the core (now at a radius $r \sim 0.1 p c$ ). This is the start of the protostellar collapse phase.

Hydrodynamic calculations by Bodenheimer, Yorke, Różyczka, and Tohline (1990), and by Laughlin and Bodenheimer (1991), indicate that when a collapse starts from a uniformly rotating, spherical volume of gas with a density distribution $\rho \sim r^{-2}$, a central object surrounded by a thick disk forms. Hydrodynamic 
calculations by Boss $(1986 ; 1991)$ indicate that the protostellar collapse, modelled initially by a uniform density sphere, begins by becoming centrally condensed while flattening due to the rotation of the model. At some point during the formation of a disk-like structure, perturbations introduced at the start of the simulation may grow significantly, resulting in fragmentation of the system. In Boss's simulations, the resulting angular momentum of the fragments is usually an order of magnitude smaller than the initial angular momentum of the model, which is consistent with the observed properties of long period binaries. A number of additional calculations also indicate that fragmentation can occur during the earliest phases of protostellar collapse (Bodenheimer, Tohline, and Black 1980; Boss 1980; Bodenheimer and Boss 1981; Miyama, Hayashi, and Narita 1984; Monaghan and Lattanzio 1991). One cannot conclude, however, that fragmentation always occurs during the protostellar collapse phase. Indeed, for a variety of conditions, fragmentation can be suppressed (Boss 1986; Safronov and Ruzmaikina 1978; Ruzmaikina 1988). The result, without fragmentation, appears to be a relatively massive equilibrium disk surrounding a central object. We suspect, as do other research groups (cf., Shu, Adams, and Lizano 1987), that certain dynamical instabilities arising in these disks can lead to disk fragmentation, and that equilibrium disk fragmentation may be the principal mechanism by which short period binary stars form. This has not yet been demonstrated, however, owing in large part to the multidimensional, nonlinear, dynamical nature of the problem. It is on this general problem that the efforts of this dissertation are focused.

While the focus of this work is primarily an analysis of the stability of protostellar disks, our results are potentially relevant to a broader class of physical systems. Linear stability analyses and computational simulations are often employed both in studies of the stability of accretion disks around evolved, compact objects (eg., 
white dwarfs and neutron stars) and in the study of protostellar disks. The principal difference is one of scaling - the simulations performed in this work, when parameters are appropriately scaled, apply equally well to any gaseous, astrophysical disk system. Hence, we will often refer to the disks under study by the more generic phrase "accretion disks."

\subsection{Relationship to Previous Studies of Disk Stability}

In the past, studies of the properties and relative stability of equilibrium disks have focused primarily on geometrically thin disks, not thick disks. In part, this is because particles with little thermal support orbiting about a dominant, central point-mass - that is, in a "Keplerian potential," $\Phi \propto 1 / r$ - assume a planar disk structure (eg., Saturn's rings), and that most researchers have become accustomed to using Keplerian potentials in theoretical work. In such disks, the material orbits with an angular velocity profile - or "rotation law" - of the form

$$
\Omega \propto r^{-3 / 2} ; j \propto r^{1 / 2}
$$

where $\Omega$ is the orbital frequency, and $r$ is the orbital separation between the particle and the central point-mass. Hence, most analyses to date have been twodimensional, linear analyses using Keplerian or near-Keplerian potentials to define the rotation law of the system. The protostellar disks that have formed from the collapse calculations of Laughlin and Bodenheimer (1991) geometrically resemble models that obey a rotation law derived from the constraint of uniform specific angular momentum - that is, the disks have vertical thicknesses comparable to their radial extent. (These geometrically thick structures resemble tori more than the flattened structures that generally come to mind when the word "disk" is used; because we are interested in geometrically thick systems, we shall use the terms "torus" 
and "disk" somewhat interchangeably). From a purely theoretical standpoint, the addition of the third dimension complicates a linear treatment considerably. Only recently have the equilibrium properties and stability of geometrically thick disks been studied (Papaloizou \& Pringe 1984; Narayan 1990; Frank \& Robertson 1988; Goodman \& Narayan 1988; Hachisu, Tohline, \& Eriguchi 1987, 1988; and Tohline \& Hachisu 1990; see also Tohline 1991). The stability of three-dimensional disk structures must be undertaken primarily from a computational approach, the work presented in this dissertation being a prime example of a somewhat experimental approach to the problem.

An additional complication that arises in linear treatments of disk stability is the mass of the disk structure. It is convenient in linear studies to ignore the gravitational effects of the mass that exists within the disk; this results in linear analyses that effectively examine only zero-mass disks (the reader is referred to Kojima 1986 for one detailed linear treatment of such disks, as well as to a review article by Narayan 1990). However, observations indicate that newly unveiled, lowmass protostars $\left(\lesssim 1 M_{\odot}\right.$ ) are frequently accompanied by disks and that these disks can have masses comparable to the mass of the central star, itself (Adams, Lada, \& Shu 1987; Strom et al. 1989; Beckwith et al. 1990). Hence, we know that dynamically stable systems with disk-to-central-object mass ratios approaching unity do exist. From a purely theoretical point of view, it is not unreasonable to expect that most of the cloud material that participates in free-fall collapse during star formation will either form or fall onto a rotationally supported disk, rather than land directly onto a thermally supported, central object. In the earliest numerical simulations of rotating cloud collapse in which fairly smooth initial cloud structures were considered (Larson 1972; Black \& Bodenheimer 1976; Tohline 1980; Boss \& Haber 1982), no central object formed from the initial collapse at all. Instead, a fully 
self-gravitating, toroidal disk structure formed. When Norman, Wilson \& Barton (1980) carefully followed the innermost regions of such collapses, they found that, depending on the cloud's initial angular momentum distribution, a small fraction of the cloud's mass can fall directly to the cloud center (presumably forming a small, pressure-supported central object). They did not show, however, what happens to the small mass at the cloud center when, subsequently on a reasonably short time scale, the remaining high specific angular momentum cloud material falls into a surrounding disk or ring structure, thereby creating a system with a very large disk-to-central-object mass ratio. Thus, a distinction is drawn between the stability analyseg of the zero-mass disk and the more complicated analyses of the finite-mass disk.

Three-dimensional analyses of the structure and stability of self-gravitating disk structures are, to this point, few in number. The principal simulations that have been performed are those by Tohline and Hachisu (1990), using disk systems with essentially no central object present. While disks were observed to be unstable in these simulations, fragmentation was not observed. This dissertation expands upon the work of Tohline and Hachisu in an extensive survey of disk stability, taking the experimental point of view. An explicit, Eulerian, finite-difference hydrodynamic code that is accurate to second-order in both space and time has been used to evolve a large number of models forward in time in order to identify under what conditions disks become dynamically unstable to nonaxisymmetric perturbations. Chapter 2 describes the hydrodynamic code used, and how it is related to the code originally used by Tohline and Hachisu. In addition, the results of the code are compared to the results originally obtained by Tohline and Hachisu for models with a disk-to-central-object mass ratio $\sim \infty$. Chapter 3 examines the stability of models with mass ratios of 5.0, 1.0, and 0.2 , that have been evolved while enforcing 
a particular geometric symmetry in the computations. Chapter 4 examines one possible effect that the formation of a massive disk may have on the central object that it surrounds. Chapter 5 examines the stability of models with the same mass ratios as Chapter 3, but the original symmetry constraint is removed in order to allow dynamical interactions between the disk and the central object. Chapter 6 summarizes the results of the simulations in the context of star formation. The appendices give additional details concerning the computational techniques used in the hydrodynamic code as well as the techniques employed in the analysis of the resulting data. 


\section{CHAPTER 2}

\section{ISOLATED, SELF-GRAVITATING DISKS}

\subsection{Introduction}

Examining the broad question of the stability of self-gravitating accretion disks/tori requires the formulation of a concise approach to the problem. We follow, basically, the same procedure as was outlined by Tohline and Hachisu (1990) when they examined the stability of massive tori. The procedure is, first, to generate by some means a set of axisymmetric hydrostatic tori whose basic physical properties are defined by a specific set of parameters. Through the use of a hydrodynamic computer code, these models are then evolved in time. If an initial model is stable, then it should remain in its initial equilibrium state. If a model is dynamically unstable, then it should evolve away from the equilibrium state. We then measure the rate at which different initial models evolve away from equilibrium in an attempt to determine over what regions of our physical parameter space models are stable (or unstable).

In this present study, the initial axisymmetric disk models are generated using a computer code written by fellow graduate student Sajed Andalib (1992), and based on Hachisu's Self-Consistent-Field (HSCF) method (Hachisu, 1986). The parameter space to be examined must be limited, as we can specify a variety of accretion disk systems with widely varying physical properties. For our study, we find it useful to categorize systems by a limited number of variables which define the physical properties of an equilibrium torus. Thus, an initial model is generated by specifying 4 dimensionless parameters: 


$$
\begin{aligned}
& -M_{d} / M_{c} \\
& -q, \\
& -n, \\
& -r_{-} / r_{+}
\end{aligned}
$$

These four parameters are, respectively, the disk-to-central-object mass ratio $\left(M_{d} / M_{c}\right)$, the exponent governing a prescribed rotation law $\left(\Omega \propto r^{-q}\right)$, the polytropic index identifying the chosen barotropic equation of state $(n)$, and the ratio of the inner $\left(r_{-}\right)$to outer $\left(r_{+}\right)$radii of the torus as specified in the equatorial plane of the system. Sometimes it is more useful for us to categorize systems through the ratio of rotational kinetic energy to gravitational potential energy $(T /|W|)$ as opposed to the ratio of inner-to-outer radii. (In previous work by Kojima (1986), $T /|W|$ was not used as a parameter for the tori; the ratio of the inner-to-outer disk edges was used instead.) We shall see that these two parameters are related. Given the four above parameters, Andalib's program generates an axisymmetric torus in hydrostatic equilibrium. It then outputs an appropriate data set defining the model in a form acceptable by our hydrodynamic code, and identifies other parameters of the equilibrium model such as the radial position at which the pressure maximum occurs $\left(r_{0}\right)$, and the corresponding rotational frequency $\left(\Omega_{0}\right)$. Figure 2.1 displays a meridional cross-section through a typical model, identifying some of these relevant quantities. Andalib's hydrostatic model is generated on a discrete cylindrical grid with the axis of the coordinate system coinciding with the axis of symmetry of the model and the central object is treated strictly as a point mass that contributes a static gravitational potential of the form $\Phi_{c}=-G M_{c} / r$. For a given set of parameters, the resulting model is unique in dimensionless units. (Further specification of a particular central object mass, $M_{c}$, and an outer radius, $r_{+}$, in dimensional units results in a unique physical model. See Appendix C.) We generate a series 


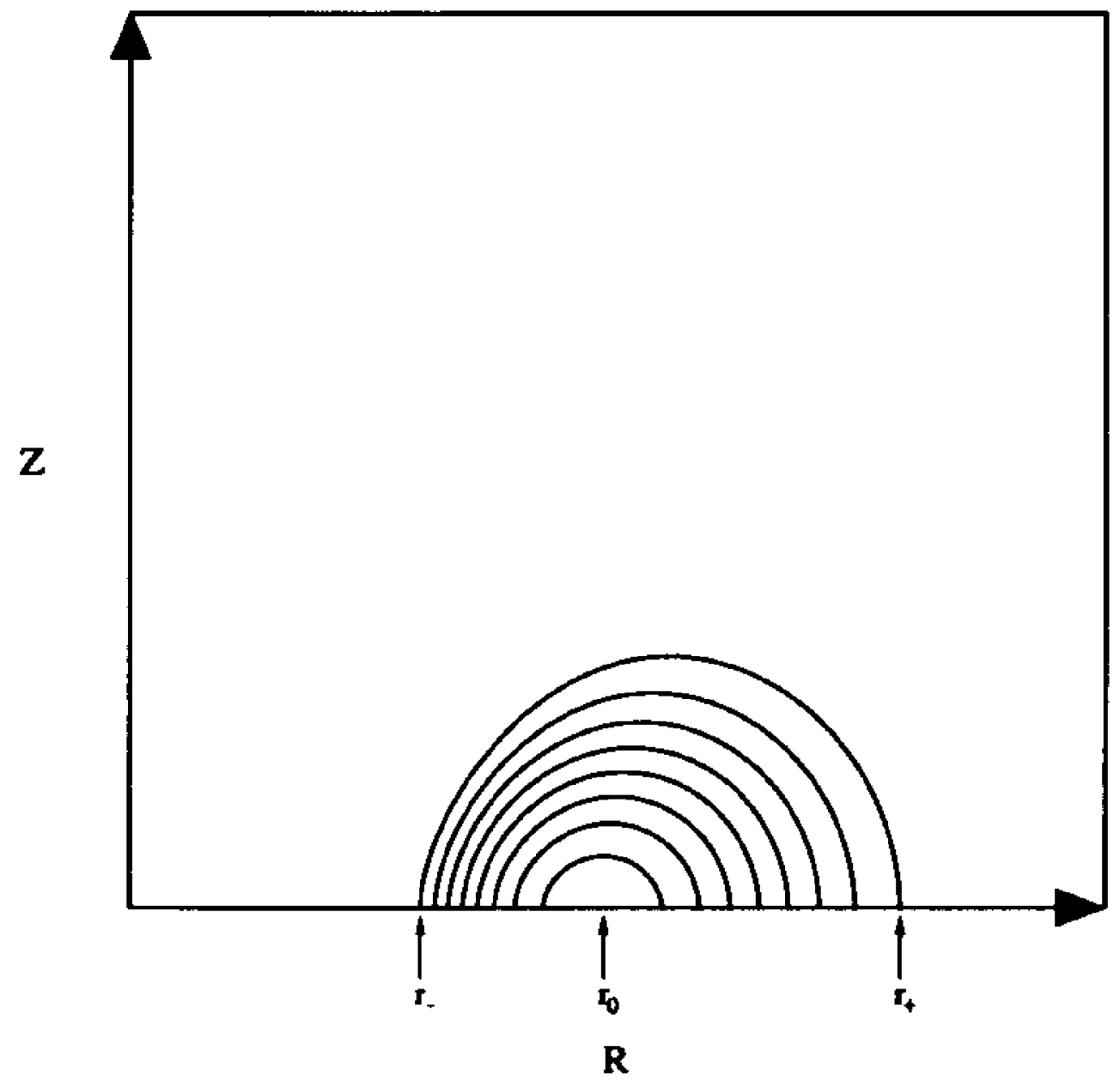

Figure 2.1: Isodensity contours of a meridional cross-section through a typical thick disk model, identifying the location of the inner and outer radii $\left(r_{-}\right.$and $\left.r_{+}\right)$, and the location of the pressure maximum $\left(r_{0}\right)$.

of models holding all parameters constant with the exception of the ratio $r_{-} / r_{+}$in order to span a range of $T /|W|$ values.

The particular hydrodynamic computer code (or hydrocode, as it is known) that is used to evolve each initial model forward in time has a fairly long history (see Tohline 1978, Williams 1988, and further discussion in $\$ 2.2$, below). It suffices at this point to say that each two-dimensional, axisymmetric torus is placed into the three-dimensional grid of the hydrocode and each three-dimensional grid 
cell is subjected to a random density perturbation $\left(\delta \rho / \rho \sim 10^{-8}\right)$. The resulting perturbed model is then evolved in discrete time intervals (or time-steps) through the use of this hydrocode. In the course of evolving the model, at each time-step a discrete spatial Fourier transform is performed on the density distribution in annular rings on the equatorial plane, yielding a modal decomposition of any resulting deviations from the equilibrium model's initial axial symmetry. The mode number, $m$, identifies in a very general sense, the shape of a distortion. For example, the Fourier amplitude of the $m=0$ mode indicates simply the azimuthally averaged value of the density in the annular ring; any amplitude in the $m=1$ mode indicates a shift of the center of mass of the model; and the $m=2$ mode indicates a bar-like distortion. The amplitude and phase angle orientation of a particular Fourier mode, examined over time, allow us to measure the growth rate and pattern speed of a developing unstable mode. Given that the initially introduced perturbation is of a random nature, no specific mode is prejudiced. We evolve each unstable model until the growth rate and pattern speed of developing modes are clearly defined. One computing session is typically 2000 time-steps in length and, when performed with a radial/vertical/azimuthal resolution of $64 / 32 / 64$ grid zones, takes approximately 9 hours of CPU time on an IBM3090/600J.

If a model has a significant growth rate, another initial model is selected and its evolutionary behavior is examined in an attempt to determine what value of $T /|W|$ determines the marginally unstable model. If a model has a low growth rate after one computing session, the evolution is continued through a number of rotation periods ( $t_{\text {rot }} \equiv 2 \pi / \Omega_{0}$, where $\Omega_{0}$ is the rotational frequency measured at the pressure maximum) until such time as the growth rate can be accurately determined. 


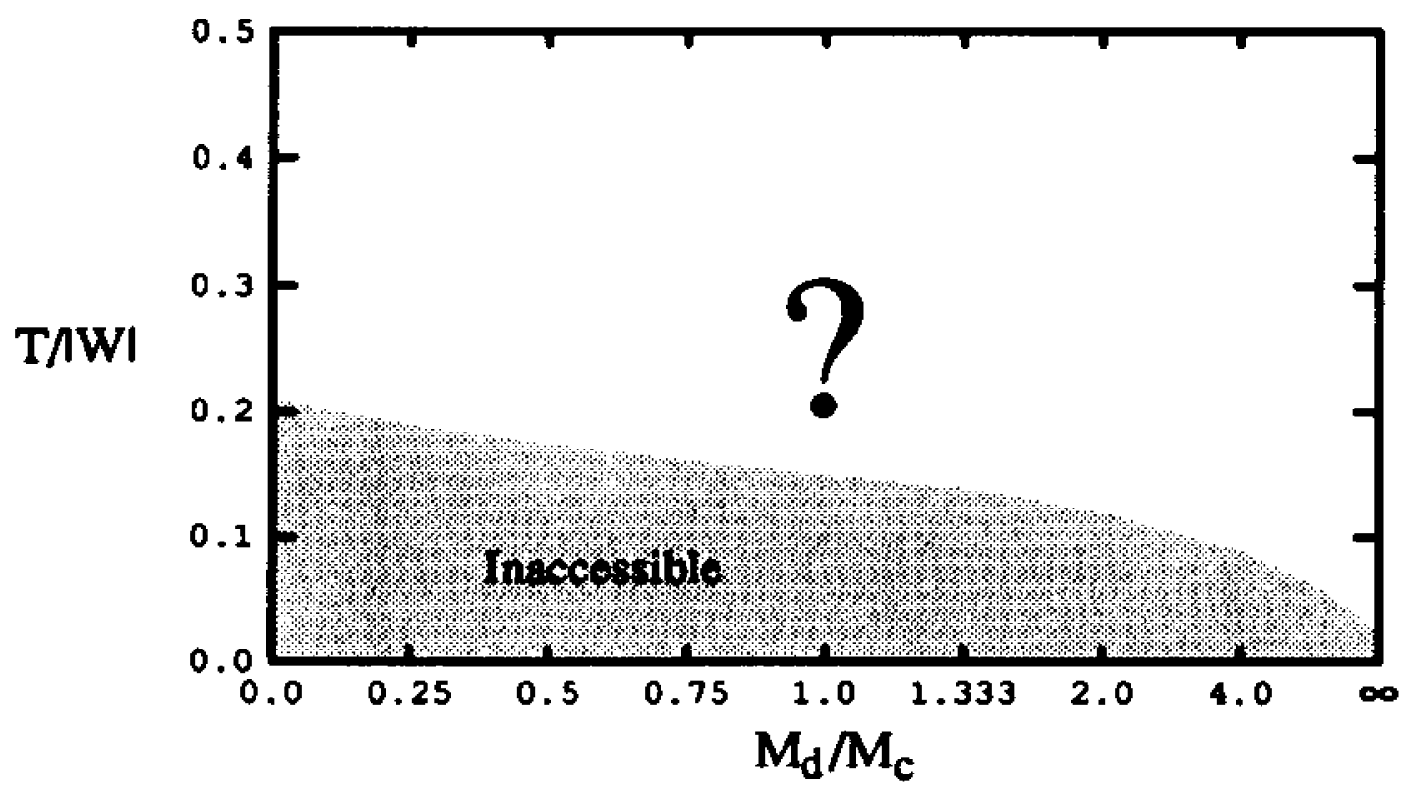

Figure 2.2: A template upon which summarized results will be illustrated as the chosen parameter space is examined. Computational constraints prohibit the evolution of extremely thick models - those having $r_{-} / r_{+}<$ 0.034; this inaccessible region is shaded.

The general goal is to determine the values of $T /|W|$ that identify marginally unstable models for a variety of mass ratios, rotation laws, and equations of state. In practice, due to the amount of computing time needed to follow the evolution of a single model, the present study is limited to models having a single polytropic equation of state ( $n=3 / 2)$, and, with few exceptions, a single rotation law ( $q=2.0$ ). By limiting the study to two variable parameters $-T /|W|$ and $M_{d} / M_{c}$ - we can indicate all the critically unstable models by plotting the value of $T /|W|$ versus mass ratio, essentially filling in the diagram indicated by Figure 2.2. [ Note: Because current computational constraints prevent us from analyzing extremely thick tori - models having $r_{-} / r_{+}<0.034-$ there is a region in the $T /|W|-M_{d} / M_{c}$ plane that is inaccessible to the present study. This inaccessible region has been shaded in Figure 2.2.] By analyzing the stability of a number of massive tori - specifically, 
systems with $M_{d} / M_{c} \approx \infty$, and, hence, essentially no central object - Tohline and Hachisu (1990, henceforth referred to as TH90), have provided the first step toward a realization of this goal. Using a hydrocode that is substantially improved over the TH90 code - both in computational performance and physical accuracy - we first repeat the TH90 analysis of the stability of massive disks.

\subsection{Hydrodynamic Code Comparison}

The code used in this work is an extensive modification of the code originally used in TH90. Both hydrodynamic codes solve a set of fuid equations which relate the fluid density, $\rho$, velocity, $v$, pressure, $P$, and gravitational potential, $\Phi=\Phi_{c}+\Phi_{d}$, over discrete intervals in time. These equations include the continuity equation,

$$
\frac{d \rho}{d t}+\rho \nabla \cdot \mathrm{v}=0
$$

the equation of motion,

$$
\rho \frac{d \mathbf{v}}{d t}=-\nabla P-\rho \nabla \Phi
$$

Poisson's equation,

$$
\nabla^{2} \Phi_{d}=4 \pi G \rho
$$

and an equation of state, given simply as

$$
P=K \rho^{\gamma}, \gamma \equiv 1+\frac{1}{n}
$$

The original code used in TH90 is a first-order-accurate, 3-D hydrodynamic code first written by Tohline $(1978 ; 1980)$, the order of the code being defined by the leading error term in a finite difference approximation to the fluxing component of the relevant fluid equations. As explained by Tohline and Hachisu, the first-order code exhibits a certain amount of numerical diffusion which, among other things, 
acts as a damping agent and tends to stifle the development of unstable, nonaxisymmetric modes. Unstable modes with reasonably fast growth rates were observable in TH90 experiments, but a "correction factor" had to be added to the measured growth rates in order to compensate for the effects of numerical diffusion and to deduce the real growth rates of unstable modes. Because of the hydrocode's inherent damping, marginally unstable models could not be precisely identified in TH90. For the present study, the major enhancement to the TH90 code is an upgrade from the first-order-accurate, donor cell fluxing scheme to a Lax Wendroff-van Leer fluxing scheme that is fully second-order in both space and time (van Leer 1976; van Albada, van Leer, and Roberts 1982; van Albada 1985). The second-order code exhibits a marked decrease in the effects of numerical diffusion. As such, real growth rates should be measurable directly (without the need to employ a "correction factor") and the new hydrocode should allow modes with very low growth rates to be studied. In addition, the new hydrocode has been substantially restructured and entire sections rewritten in order to use IBM's Parallel Fortran Program to take advantage of both the vectorization and multiple processing capabilities of the IBM3090 series of mainframe computers (see Appendix A for a more extensive discussion of the new hydrocode).

Another basic difference in the computer codes used lies in the program that generates the initial equilibrium models. While both codes use the Hachisu selfconsistent-field method (Hachisu 1986), the version of this method used in TH90 generates initial models on a spherical coordinate mesh, requiring an interpolation to the cylindrical coordinate mesh which is used by the hydrodynamic code. This differs from the code written by Andalib (1992) and used here, which generates initial models using the same cylindrical coordinate mesh as the hydrodynamic code, thus eliminating the interpolation step. In evolutions not directly related to 
this work, we have seen that models generated with the original HSCF code tend to "bounce" or "breathe" slightly when run in the new, second-order hydrocode, whereas equivalent models generated by Andalib's HSCF code do not exhibit this behavior. This behavior must be due to the interpolation between the two coordinate systems. These axisymmetric oscillations about the equilibrium state are not physically interesting in this study, so we use Andalib's HSCF code in preference to the one used in TH90.

Given that the TH90 hydrocode has been exhaustively tested and compared against physical results from linear theory, this chapter duplicates the models used in TH90 as closely as possible in order to provide a definitive test of the accuracy and reliability of the newer, second-order code. The growth rates resulting from the second-order hydrocode should correspond closely with the "corrected" results from the first-order hydrocode if the second-order hydrocode is to be perceived as being accurate.

\subsection{Analysis of the Data}

In linear perturbation studies, it is common to express the fractional variation in the density $\delta \rho$ from axisymmetry by

$$
\frac{\delta \rho}{\rho} \propto e^{-i[w t-m \theta]}
$$

where $\omega$ is a complex frequency and, as before, $m$ is the azimuthal mode number. In such a form, then, for each mode, the growth rate and pattern speed of the density perturbation are identified from $\operatorname{Im}(\omega)$ and $\operatorname{Re}(\omega)$, respectively. Within the hydrocode, a Fourier transform is performed on the density distribution in the course of solving the discretized Poisson equation. As such, the density at any 
instant in time is expressed as a Fourier series,

$$
\rho(r, \theta, z, t)=\frac{1}{2} c_{0}(r, z, t)+\sum_{m=2}^{L_{m}=} c_{m}(r, z, t) \cos \left[m \theta+\phi_{m}(r, z, t)\right]
$$

The correspondence between $\omega, \phi_{m}$, and $c_{m}$ is given by

$$
\begin{gathered}
R e(\omega)=\frac{d \phi_{m}}{d t} \\
I m(\omega)=\frac{d l n c_{m}}{d t}
\end{gathered}
$$

(Williams and Tohline 1987).

Instead of directly tabulating the growth rate for a particular azimuthal mode, and that mode's corresponding pattern speed, we follow the convention of Kojima (1986) and calculate two equivalent parameters. The first parameter, $y_{1}(m)$, js related directly to the pattern speed, and is given by:

$$
y_{1}(m) \equiv\left[\frac{\operatorname{Re}(\omega)}{\Omega_{0}}-m\right]
$$

The second parameter, $y_{2}(m)$, is related to the growth rate:

$$
y_{2}(m) \equiv \frac{I m(\omega)}{\Omega_{0}}
$$

These can be expressed in a more useful form by substituting expressions (2.7) and (2.8) for $\operatorname{Re}(\omega)$ and $I m(\omega)$, using the normalized mode amplitude $D_{m} \equiv c_{m} / c_{0}$ (which we output as a function of time from the hydrocode), and making use of the definition of a rotation period, $t_{\text {rot }} \equiv 2 \pi / \Omega_{0}$ :

$$
\begin{gathered}
y_{1}(m)=\left[\frac{d \phi_{m} / 2 \pi}{d\left(t / t_{\text {rot }}\right)}-m\right], \\
y_{2}(m)=\frac{1}{2 \pi} \frac{d \ln D_{m}}{d\left(t / t_{\text {rot }}\right)}
\end{gathered}
$$


The calculation of these parameters from the Fourier data resulting from a model's evolution allows us to determine other useful physical information. For example, for a given mode $m$, the relationship between the pattern speed $\Omega_{p}$ and $d \phi / d t$,

$$
\Omega_{p}=\frac{1}{m} \frac{d \phi_{m}}{d t}
$$

allows us to easily calculate the pattern speed from $y_{1}$ :

$$
\frac{\Omega_{P}}{\Omega_{0}}=\frac{y_{1}(m)}{m}+1
$$

Also, the corotation radius $r_{c r}$ is the position where the fluid rotational frequency is equal to the pattern speed. So, using the imposed rotation law $\Omega=\Omega_{0}\left(r / r_{0}\right)^{-q}$, the corotation radius $r_{c r}$ relative to the radius of the pressure maximum $r_{0}$ can be determined as

$$
\frac{r_{c r}}{r_{0}}=\left[\frac{y_{1}(m)}{m}+1\right]^{-1 / 9} .
$$

A third potentially useful piece of information is the location of the inner and outer Lindblad resonances in an unstable disk. In a particle syatem, if a disk particle deviates slightly from a purely circular orbit, it will experience epicyclic motion about a guiding center which follows the circular orbit (see, eg., Binney and Tremaine 1987). The square of the epicyclic frequency is given by

$$
\kappa^{2}=\frac{1}{r^{3}}\left[\frac{d}{d r}\left(r^{2} \Omega\right)^{2}\right] .
$$

The Lindblad resonance, then, is a resonance between $\Omega_{p}-\Omega$ and the epicyclic frequency, given by

$$
m\left(\Omega_{p}-\Omega\right)= \pm \kappa
$$

Through the use of the rotation law in our models, positions of the inner and outer Lindblad resonances are given by

$$
\frac{r_{L B}}{r_{c r}}=\left[\frac{m}{m \mp \sqrt{4-2 q}}\right]^{-1 / q},
$$

where the outer (inner) Lindblad resonance is found using the $+(-)$ sign. 


\subsection{An Idealized Model}

This dissertation involves the evolution of approximately 40 different accretion disk models. The hydrodynamic code used to evolve these models outputs an enormous quantity of data, from which values for $y_{1}(m)$ and $y_{2}(m)$ must be determined. This section is devoted to presenting the precise methods through which the growth rate and pattern speed parameters are determined from the normalized Fourier mode amplitudes, $D_{m}$, and the Fourier phase parameter, $\phi_{m}$, that are regularly output by the hydrocode at the end of a computing session.

For an exponentially growing eigenmode, the two parameters $y_{1}(m)$ and $y_{2}(m)$, expressed as in eqs. 2.11 and 2.12 , can be measured from the slopes of lines if $\phi_{m} / 2 \pi$ and $\ln D_{m}$ are plotted versus $t / t_{\text {rot }}$. (Hereafter, plots of this nature will frequently be referred to as, respectively, $\phi_{m}-t$ and $D_{m}-t$ graphs.) In our analysis of the data resulting from the hydrocode, the relevant parameters are determined in exactly this manner. In the early phase of a model's evolution, the $D_{m}-t$ graph is essentially linear. In regions where the density fluctuations are $\operatorname{small}(\delta \rho / \rho<1)$, this linear behavior is the result obtained through linear theory, and as such, is referred to as "the linear regime." At high amplitudes $(\delta \rho / \rho \sim 1)$, the behavior is more complex, the $D_{m}-t$ graph becoming decidedly nonlinear. This region is referred to as "the nonlinear regime." From a computing standpoint, the hydrocode uses single precision calculations on the IBM3090, so fluctuation amplitudes $\delta \rho / \rho \leqq 10^{-8.5}$ cannot be physically meaningful. For this reson, the normalized amplitude $D_{m}$ is not included in our determination of $y_{1}(m)$ if $\ln D_{m} \lesssim-12.7$.

An idealized example of how the growth rate and pattern speed parameters are determined using $D_{2}-t$ and $\phi_{2}-t$ graphs can be illustrated using Figure 2.3a. In this idealized case, we have created an ad hoc $m=2$ mode that displays purely 

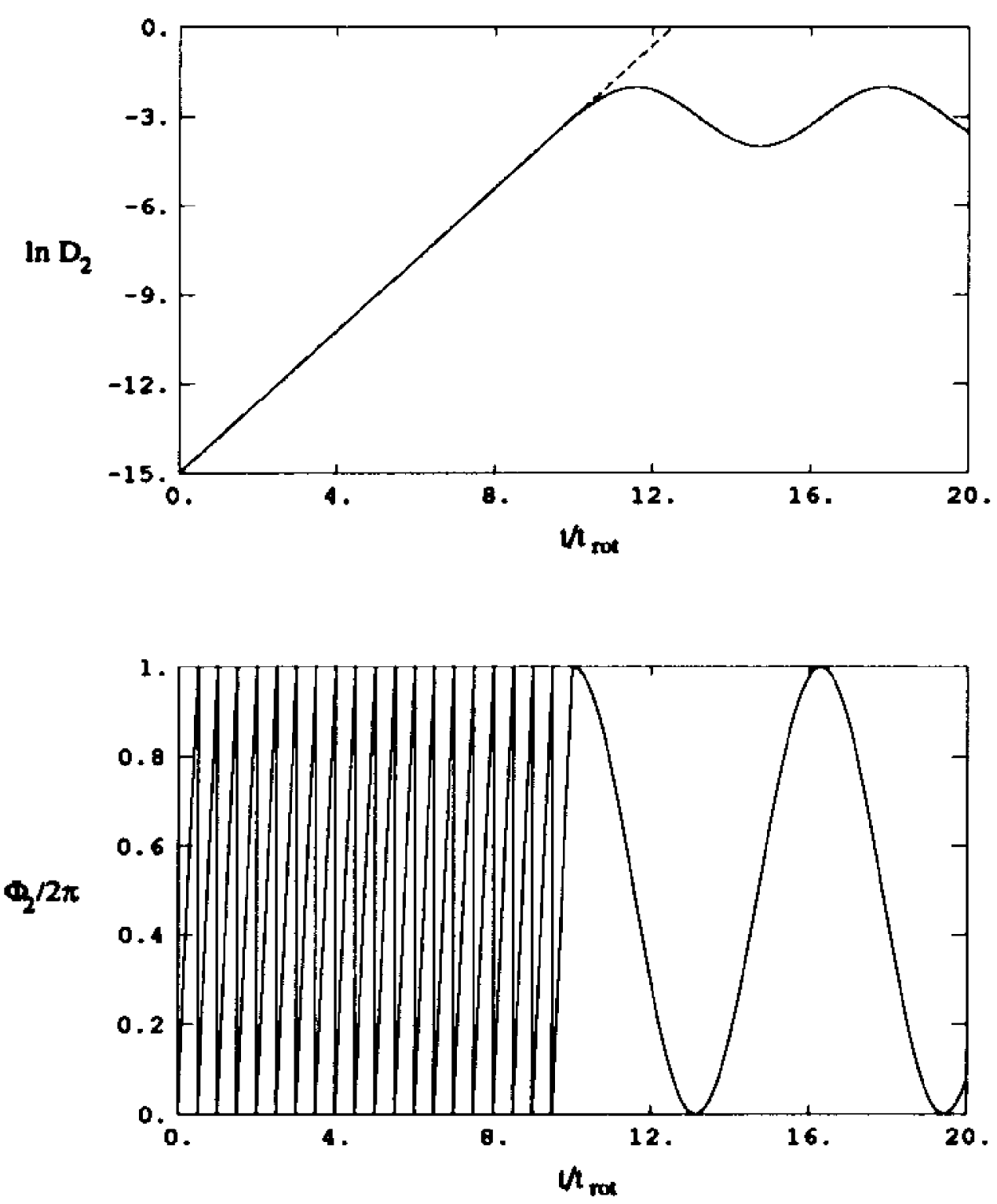

Figure 2.3a: The upper panel illustrates, for an idealized model, the logarithm of the $m=2$ Fourier amplitude $D_{2}$ as it evolves as a function of time. The dashed line indicates a linear least squares fit to the data during the linear-amplitude portion of the evolution; the $y_{2}(2)$ parameter is obtained from the slope of this dashed line. If higher order modes were present, they would be indicated by a succession of dashed lines, the spacing of the dashes increasing as mode number increases; in this idealized model, higher order modes are assumed to have zero amplitude. The lower panel illustrates the phase behavior of the $m=2$ Fourier mode as a function of time, from which the $y_{1}(2)$ parameter is obtained. 
exponential growth for 10 rotation periods. After this time, having reached an amplitude $\ln D_{2}=-3$, the mode exhibits some form of more complicated, nonlinear behavior. (The exact form of the nonlinear growth behavior is not of principal importance in this work so it is illustrated in only a very qualitative manner in Figure 2.3a.) The slope $d l n D_{2} / d\left(t / t_{\text {rot }}\right)$ can be obtained by measuring points with a ruler (as has been done in the past), or by performing a linear least squares fit to the region of interest. Throughout this dissertation, we have used Mathematica to read in the data to create the $D_{2}-t$ and $\phi_{2}-t$ plots, and to make a linear fit to a specified portion of the $D_{2}-t$ plot. (Appendix B displays the Mathematica notebook which handles this analysis.) The $y_{2}(2)$ parameter is, then, simply the resulting slope divided by $2 \pi$. For our idealized example, $y_{2}(2)=0.191$. The $y_{1}(2)$ parameter is not obtained "automatically" by Mathematica as the slope of a portion of the $\phi_{2}-t$ plot. Instead, over a given interval of time $\delta t / t_{\text {rod }}$, a number of cycles is counted, corresponding to $\delta \phi / 2 \pi$. The measured ratio $\left(\delta \phi_{2} / 2 \pi\right) /\left(\delta t / t_{\text {rot }}\right)$ is used as the value of $\left(d \phi_{2} / 2 \pi\right) /\left(d t / t_{\text {rot }}\right)$ in Eq. 2.11. In our idealized example, 16 cycles occurred over 8 rotation periods for the $m=2$ mode, yielding $y_{1}(2)=0.0$ and, by Equation (2.15), $r_{\mathrm{cr}} / r_{0}=1$.

If the $y_{2}(2)$ parameter determined from a given evolution is nearly zero, the model may be deemed the "marginally unstable" model, defining the juncture in $T /|W|$ below which models are stable, and above which models are unstable to nonaxisymmetric instabilities. Correspondingly, a $y_{1}(2)$ parameter of zero indicates that the unstable eigenfunction exhibits a pattern speed that equals the rotational frequency of the disk exactly at its pressure maximum.

The upper panels in Figure 2.3b display, at two different times in the evolution, the radial eigenfunction of our "jdealized" $m=2$ mode in a phase vs. radius plot $\left(\phi_{2}-r\right)$. In general, the time periods we choose to display will correspond 

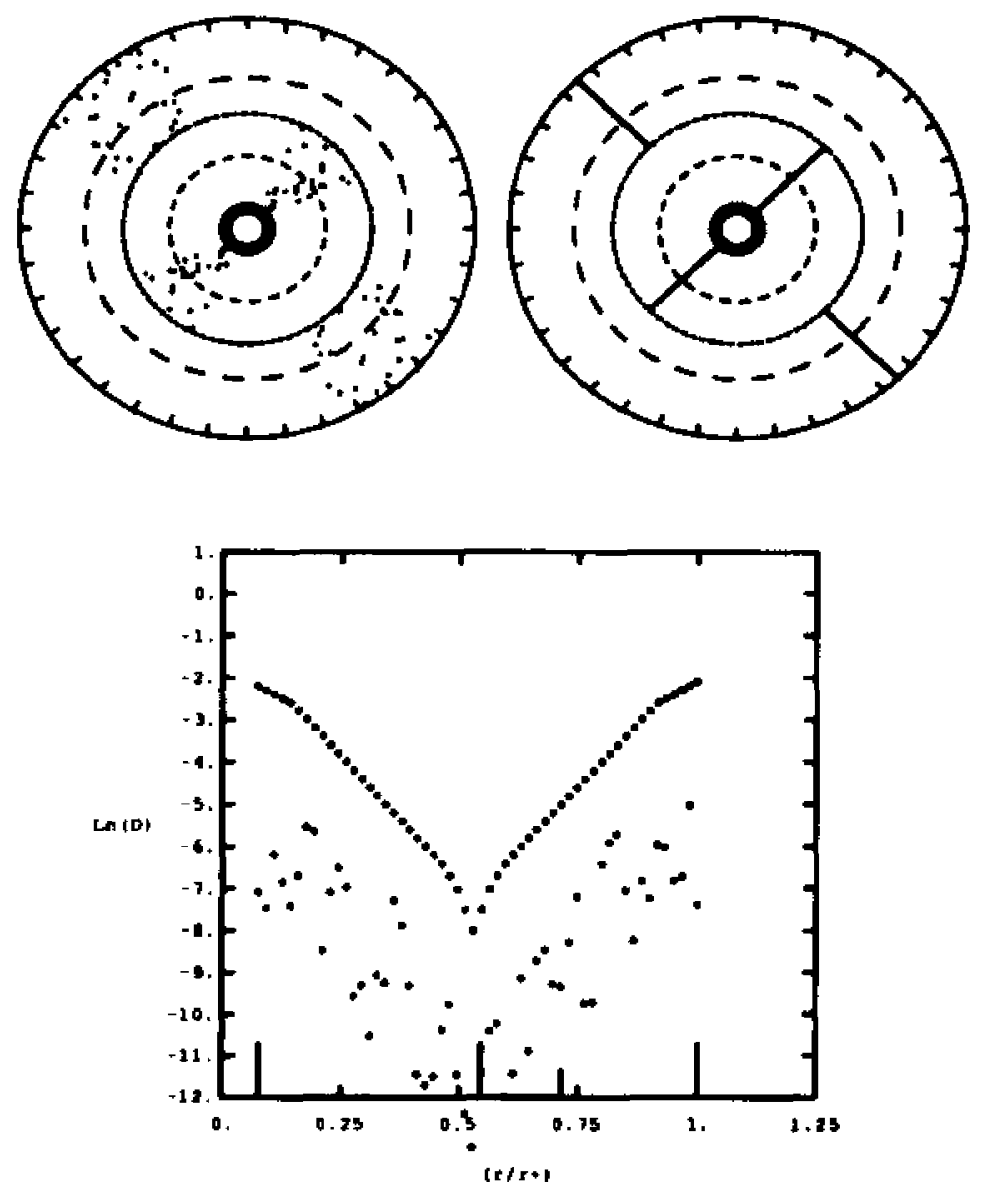

Figure 2.3b: The upper panels illustrate the radial eigenfunction $\left(\phi_{2}-r\right.$ behavior) of the $m=2$ mode instability of an idealized model at two points in time. The panel on the left corresponds to the midpoint of the evolution; the panel on the right occurs near the endpoint of the evolution. Tick marks are placed at $r_{-}$and $r_{+}$at 10 degree intervals. Dashed circles placed between the inner and outer radii indicate the positions of the pressure maximum $r_{0}$ (the middle of the three indicated here), the corotation radius $r_{c r}$ (coinciding with the pressure maximum in this particular idealized example), the inner Lindblad resonance (between $r_{c r}$ and $r_{-}$), and the outer Lindblad resonance (between $r_{c r}$ and $r_{+}$). The data points making up the phase vs. radius behavior occur as a series of solid points. Note in particular the $\pi / 2$ shift in phase that occurs at the pressure maximum. The lower panel illustrates the amplitude of the $m=2$ mode as a function of radius $\left(D_{2}-r\right)$ at two points in time. The solid points correspond to the point in time at which the upper, left $\phi_{2}-r$ plot is taken; the hollow points correspond to the time at which the upper, right $\phi_{2}-r$ plot is taken. Long vertical lines on the radial axis indicate the position of $r_{-}$, $r_{0}, r_{c r}$ (coinciding with $r_{0}$ in this case), and $r_{+}$. The shorter vertical line indicates the position of the outer Lindblad resonance. Note in particular the occurrence of a minimum in amplitude at $r_{0}$. 
to the midpoint and endpoint of an evolution. The radial positions of the pressure maximum, $r_{0}$, the corotation radius, $r_{c r}$, and the inner and outer Lindblad resonances, $r_{j \mid r}$ and $r_{0 / r}$, are illustrated as a series of dashed circles in the $\phi_{2}-r$ figure. Also, tick marks are placed every 10 degrees around the two solid circles that identify the inner and outer edges of the toroidal disk model. Note that with a Keplerian rotation law $(q=1.5)$, the Lindblad resonances will normally straddle the corotation point, whereas with a $q=2.0$ rotation law, the Lindblad resonances and the corotation radius will coincide. The bottom panel of Figure $2.3 \mathrm{~b}$ depicts the amplitude of our idealized $m=2$ mode as a function of radius $\left(D_{2}-r\right)$. A minimum occurring near $r_{0}$ is typical of most unstable eigenfunctions that we have analyzed in this study.

The structure illustrated in the idealized $\phi_{2}-r$ plot (Figure 2.3b) is typical of most evolutionary sequences in which imposed symmetries allow only even azimuthal modes to develop. The unstable eigenfunction exhibits an $m=2$ behavior, with a phase shift of $\pi / 2$ occurring near the pressure maximum of the model. This phase shift of $\pi / 2$ can be understood easily as follows (for a related discussion, see Tohline 1980, particularly Figure 5 of that paper). Consider a perfectly axisymmetric torus that is distorted into a slightly elliptical shape. A Fourier analysis of its density distribution that is performed on the original cylindrical coordinate mesh will produce the phase orientation of the density maximum for each radial position in the model that exhibits the following character: At radii $r<r_{0}$, the azimuthal maximum in the density will occur along the aemi-minor axis of the elliptical torus. At radii $r>r_{0}$, however, the azimuthal maximum in the density occurs at the semi-major axis of the model. For the initial, axisymmetric models which we have evolved with our hydrodynamic code, it is important to note that the radial density maximum occurs precisely at the pressure maximum. Hence, in the eigenfunction 
plots under discussion, the position of the azimuthal density maximum should undergo a shift of $\pi / 2$ in the vicinity of the indicated pressure maximum of the model if the amplifying $m=2$ distortion is basically just a global ellipsoidal distortion of the disk.

\subsection{Selected Massive Models}

In their study of massive, self-gravitating disks/tori $\left(M_{d} / M_{c} \approx \infty\right)$, Tohline and Hachisu (1990) found that systems having $T /|W| \lesssim 0.15$ were dynamically stable against axisymmetric and nonaxisymmetric perturbations. Tori with $T /|W| \gtrsim 0.16$ were found to be dynamically unstable. The models generated for comparison here correspond to 7 of the 8 different $T /|W|$ models examined in TH90. The data for the initial models used in this comparison is given in Table 2.1, where the model numbers have been assigned to match the corresponding model numbers used in TH90. Also indicated is the corresponding value of $T /|W|$ for the models evolved in TH90. While the correspondence between our initial models and the ones used in TH90 is not one-to-one, the differences are slight and the generated models serve well for a comparison between the two codes. Note that in the newer initial model code written by Andalib (1992), the rotation law parameter $q$ is identical to one-half of TH90's $l$ parameter. As stated earlier, the rotation law used here is $\Omega \propto r^{-q}$, so we can see that $q=2$ corresponds to a model with constant specific angular momentum, while $q=3 / 2$ corresponds to a Keplerian rotation law.

One can see from the Keplerian models in Table 2.1 the monotonic relationship between a chosen value of $r_{-} / r_{+}$and the resulting equilibrium value of $T /|W|$. As the value of $r_{-} / r_{+}$increases (the torus becomes geometrically slimmer), the value of $T /|W|$ also increases. 
During each hydrodynamic evolution, we enforce the same symmetry conditions as used in TH90. The angular region of the computational grid from 0 to $\pi$ is mapped onto the region from $\pi$ to $2 \pi$. This " $\pi$-symmetry" is enforced because one expects the center of mass of the disk/torus to remain fixed at the center of the coordinate grid. Thus, it is impossible for the $m=1$ mode to occur, and $m=2$ will be the lowest order mode of any consequence. Given the computational expense of performing these evolutions, the enforcement of $\pi$-symmetry either saves a considerable amount of computing time, or allows us to use a higher azimuthal grid resolution.

\subsection{Resulta and Comparison}

Each model was evolved through 5 to 10 rotation periods - long enough to have progressed through most of the linear-amplitude stage of each evolution. The results compare quite favorably with the first-order results, the evolutions proceeding as described in TH90. The resulting data is very clean, with essentially no unexpected behavior. Figures 2.4a through 2.10b show graphically the Fourier amplitude and phase behavior as a function of time (the $D_{m}-t$ and $\phi_{m}-t$ plots), the character of the $m=2$ distortion at different times during each model's evolution ( $\phi_{m}-r$ plot), and the amplitude of the distortions as a function of radius at these times $\left(D_{m}-r\right.$ plot). From the $D_{m}-t$ and $\phi_{m}-t$ plots, we directly measure $d l_{n} D_{m} / d\left(t / t_{\text {rot }}\right)$ and $d\left(\phi_{m} / 2 \pi\right) / d\left(t / t_{\text {rot }}\right)$, respectively, and, as described above, determine the parameters $y_{1}$ and $y_{2}$ for each model.

By way of summary and comparison, the values of these and other physical parameters of the unstable $m=2$ eigenmode are tabulated in Table 2.2 for our seven "massive" disk models. Figure 2.11 shows the two parameters $\nu_{1}(2)$ and $y_{2}(2)$ versus $T /|W|$ as measured both by our current second-order code (solid circles) 
and by the TH90 first-order code (open squares). Models \#2 - \#6 show excellent agreement between the uncorrected $y_{2}(2)$ parameter resulting from the second-order hydrocode and the $y_{2}(2)$ parameter from TH90's first-order hydrocode to which a corrective factor was added. Using these four models as a basis, we can accept the second-order code as an accurate one, if the three models which show disagreement can be adequately explained. Model \#1 differs in the $y_{2}(2)$ parameter significantly, but we note that the majority of the $y_{2}(2)$ parameter reported in TH90 is the correction factor (the correction factor for the TH90 code is given as $\sim 0.098(m / 2)^{2}$ ). Evidently, if any model near the critically unstable value of $T /|W|$ is evolved using the TH90 hydrocode, the correction factor will over-compensate for the numerical damping inherent in that code, yielding $y_{2}(2)$ parameters $\sim 0.1$ for the $m=2$ mode. The second-order code used in this study, requiring no correction factor for the $y_{2}(2)$ parameter, yields growth rate parameters much closer to zero for the critically unstable models.

One can see in Figure 2.11 that at the high end of the $T /|W|$ range, the growth rates determined from our second-order code show a marked deviation from the first-order results reported in TH90. At a value of $T /|W|=0.273$ (model \#8), it is clear that the discrepancy is due to a change in which unstable mode is the dominant one. In TH90, all the models exhibited an $m=2$ instability as the dominant mode. In the present calculation, however, model \#8 clearly exhibits an $m=4$ instability as the dominant mode (see the top panel of Figure 2.10a). This is not completely unexpected, given the results of Papaloizou and Pringle (1984) and Kojima (1986). In a linear analysis of the atability of massless tori, Kojima found that at small values of $r_{-} / r_{+}, m=1$ was the dominant mode. As $r_{-} / r_{+}$increased, the growth rate of the $m=1$ mode increased to some maximum value, and then diminished as the growth rate of the next higher mode, $m=2$, increased. The same 
characteristic behavior occurred for all modes. Analogously, our low $T /|W|$ models are unstable to a low order mode ( $m=2$ ), but as $T /|W|$ is increased, the dominant mode shifts toward the next higher mode (which is $m=4$ here, due to the imposed “-symmetry").

It would appear that the numerical diffusion inherent in TH90's first-order computational scheme was sufficient to prevent the higher order modes from growing appreciably. Hence, model \#8, evolved here with the second-order code, cannot really be compared to ita counterpart in TH90. For model \#7, with a slightly lower value of $T /|W|$, the growth of the $m=2, m=4$, and $m=6$ modes are comparable to one another for a sizeable portion of the early evolution. This behavior is unlike the results reported for model \#7 in TH90 and unlike the results of the other second-order models in our present study. Only after two full rotation periods does the $m=2$ mode emerge as the dominant mode, but with the $m=4$ and $m=6$ modes still having sizeable amplitudes. This model seems to lie near the crossover point where the $m=4$ growth rate begins to increase appreciably and the $m=2$ growth rate diminishes.

Agreement between the two hydrodynamic codes on the $y_{1}(2)$ parameter was not as good as the agreement on growth rates. No correction factors have been added to the TH90 results, as a strict first-order analysis of the errors indicates that no correction factor is required. While a correction factor is indicated for the second-order code used here (Christodoulou 1990), the magnitude of the correction is negligible (the correction factor is $\left.\sim 0.004(m / 2)^{2}\right)$. We believe this difference is not significant, however, and attribute it to the numerical diffusion inherent in the TH90 code.

Given that the majority of the evolved models yield results in good agreement with the first-order results, and that the few differences can be adequately explained, 
we can accept the second-order code as an accurate, and now proven code (at least in the linear-amplitude growth regime; we refer the reader to Appendix D for a more direct comparison of the hydrocode against the results of linear theory ). Also, there is an indication that the numerical diffusion in the first-order code cannot be completely compensated for through the simple addition of a "correction factor" to measured growth rates. Numerical diffusion in the first-order code can completely stifle the growth of physically dominant modes at high values of $T /|W|$. 
Table 2.1

Initial Model Data for $M_{d} / M_{c}=\infty$

\begin{tabular}{ccccccc}
\hline \hline TH90 Model \# & $q$ & $M_{d} / M_{c}$ & $r_{-} / r_{+}$ & $r_{0} / r_{+}$ & $T /|W|$ & TH90 T/|W| \\
1 & 2.0 & $7.55 \times 10^{5}$ & 0.203 & 0.472 & 0.171 & 0.167 \\
2 & 1.75 & $7.03 \times 10^{5}$ & 0.210 & 0.500 & 0.193 & 0.189 \\
4 & 1.5 & $6.86 \times 10^{5}$ & 0.180 & 0.490 & 0.199 & 0.195 \\
5 & 1.5 & $6.42 \times 10^{9}$ & 0.215 & 0.519 & 0.215 & 0.212 \\
6 & 1.5 & $5.84 \times 10^{9}$ & 0.260 & 0.570 & 0.232 & 0.230 \\
7 & 1.5 & $5.00 \times 10^{9}$ & 0.325 & 0.612 & 0.253 & 0.251 \\
8 & 1.5 & $4.09 \times 10^{9}$ & 0.404 & 0.670 & 0.273 & 0.271 \\
\hline
\end{tabular}


Table 2.2

m=2 Eigenmode Character, $M_{d} / M_{c}=\infty$

\begin{tabular}{|c|c|c|c|c|c|c|c|c|c|}
\hline Model & $y_{1}$ & $\overline{y_{2}}$ & $\Omega_{p} / \Omega_{0}$ & $r_{c r} / r_{0}$ & $r_{\mathrm{cr}} / r_{+}$ & $r_{i l r} / r_{0}$ & $r_{i i r} / r_{+}$ & $r_{\text {olr }} / r_{0}$ & $r_{\text {olr }} / r_{+}$ \\
\hline 1 & -0.85 & 0.079 & 0.57 & 1.31 & 0.62 & 1.31 & 0.62 & 1.31 & 0.62 \\
\hline 2 & -1.01 & 0.301 & 0.49 & 1.49 & 0.74 & 1.16 & 0.58 & 1.77 & 0.88 \\
\hline 4 & -1.00 & 0.338 & 0.50 & 1.58 & 0.77 & 1.00 & 0.49 & 2.08 & 1.01 \\
\hline 5 & -1.00 & 0.381 & 0.49 & 1.58 & 0.82 & 1.00 & 0.52 & 2.08 & 1.08 \\
\hline 6 & -0.94 & 0.394 & 0.52 & 1.52 & 0.87 & 0.96 & 0.54 & 2.00 & 1.14 \\
\hline 7 & -0.87 & 0.315 & 0.56 & 1.46 & 0.89 & 0.92 & 0.56 & 1.92 & 1.17 \\
\hline 8 & -0.16 & 0.357 & 0.91 & 1.06 & 0.71 & 0.66 & 0.44 & 1.38 & 0.93 \\
\hline
\end{tabular}



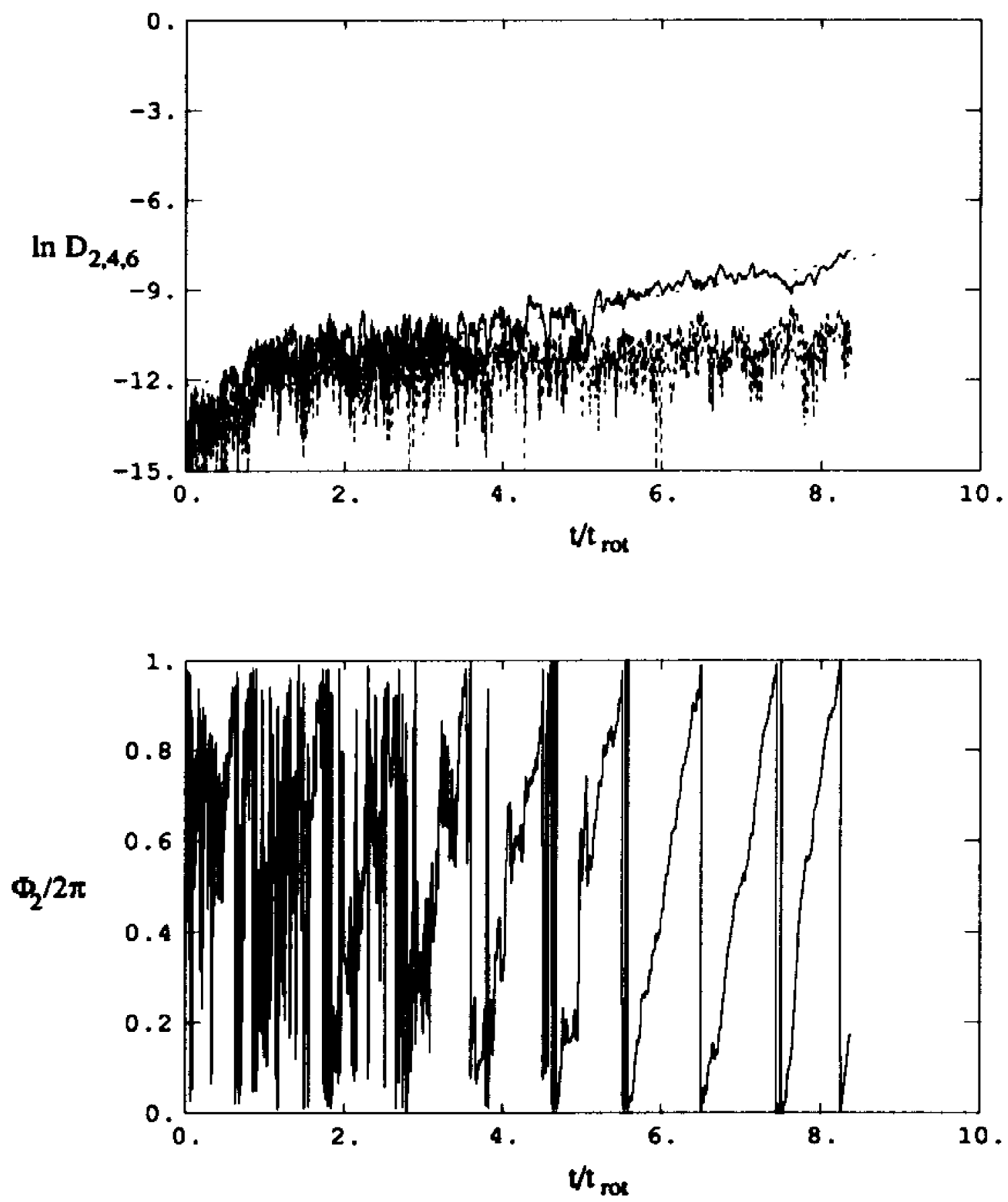

Figure 2.4a: The $m=2, m=4$, and $m=6$ Fourier amplitude behavior, and the $m=2$ phase behavior as a function of time, illustrated as in Figure 2.3a for $M_{d} / M_{c}=\infty$, model \#1. 

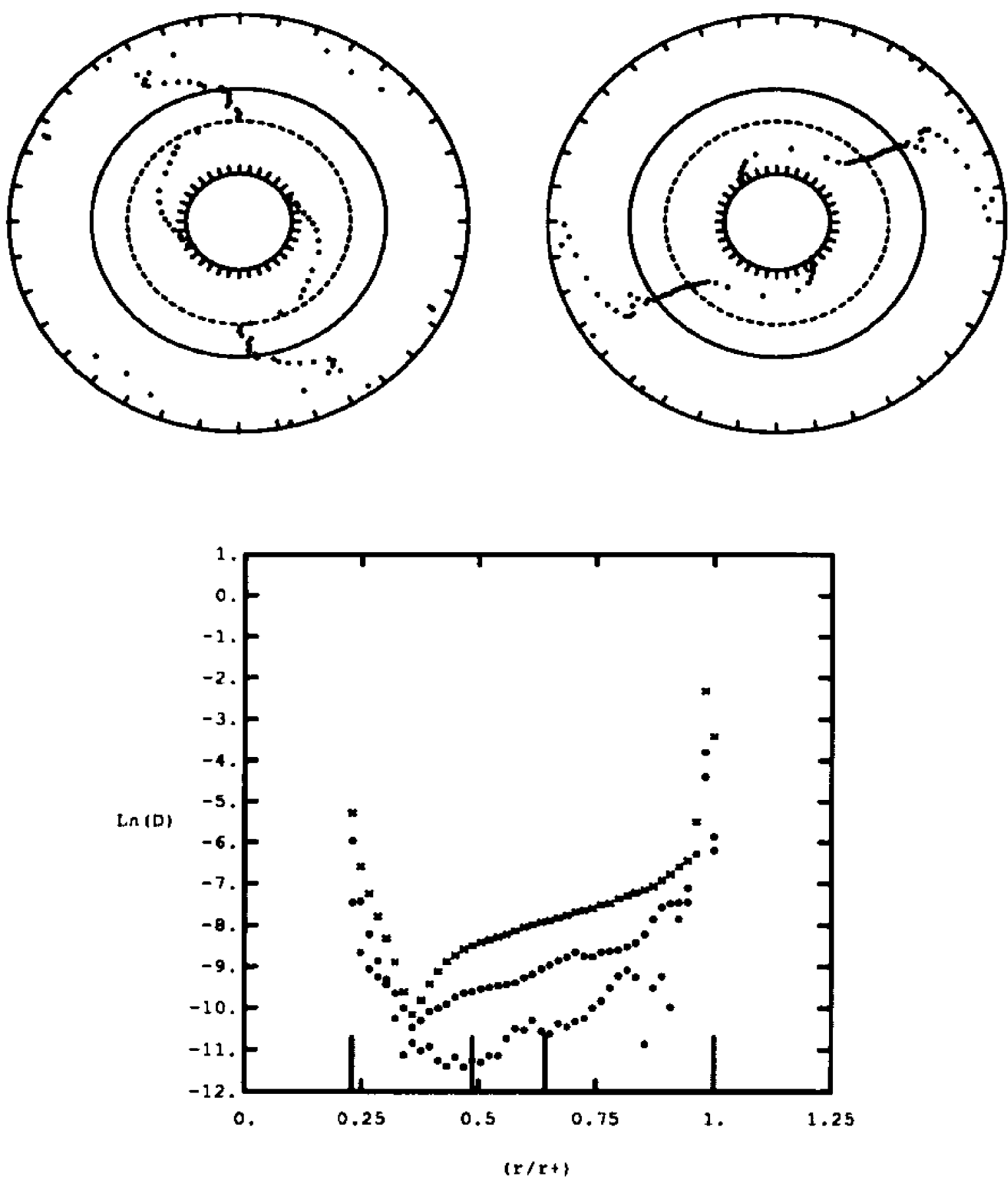

Figure 2.4b: The radial eigenfunction $\left(\phi_{2}-r\right)$ and amplitude as a function of radius $\left(D_{2}-r\right)$ of the $m=2$ Fourier mode illustrated as in Figure $2.3 \mathrm{~b}$ for $M_{d} / M_{c}=\infty$, model \#1. 

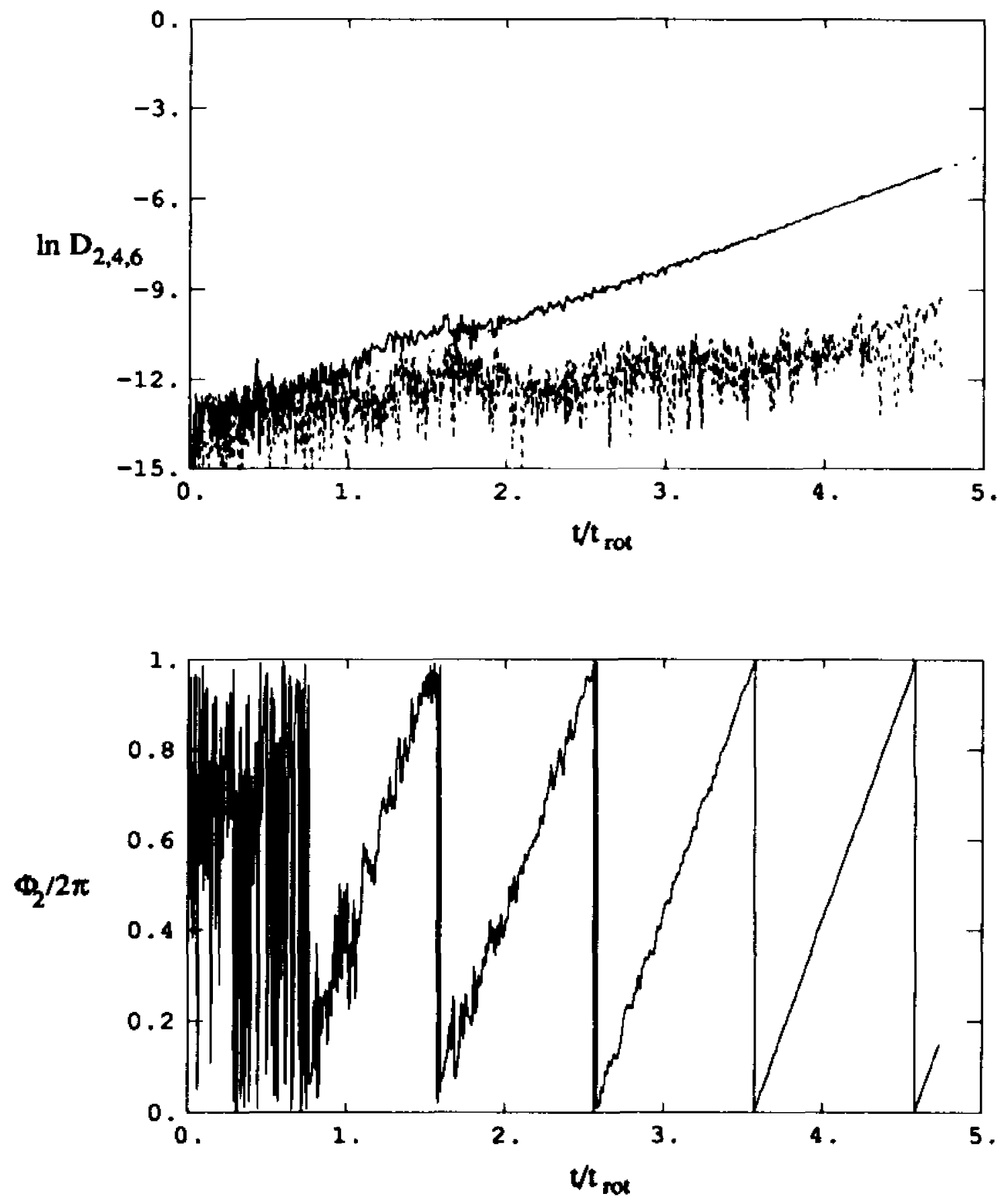

Figure 2.5a: The $m=2, m=4$, and $m=6$ Fourier amplitude behavior, and the $m=2$ phase behavior as a function of time, illustrated as in Figure 2.3a for $M_{d} / M_{c}=\infty$, model \#2. 

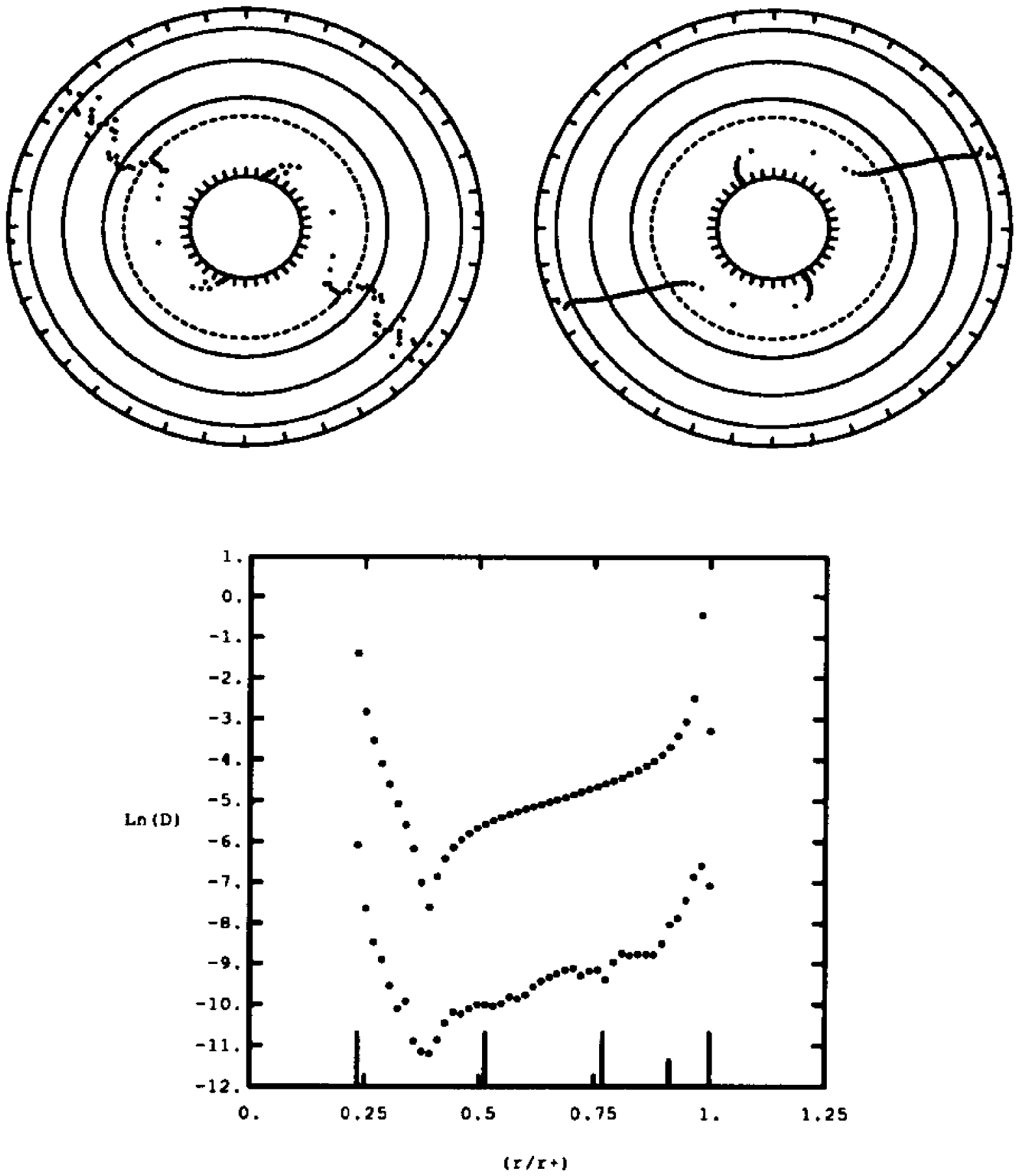

Figure 2.5b: The radial eigenfunction $\left(\phi_{2}-r\right)$ and amplitude as a function of radius $\left(D_{2}-r\right)$ of the $m=2$ Fourier mode illustrated as in Figure 2.3b for $M_{d} / M_{c}=\infty$, model \#2. 

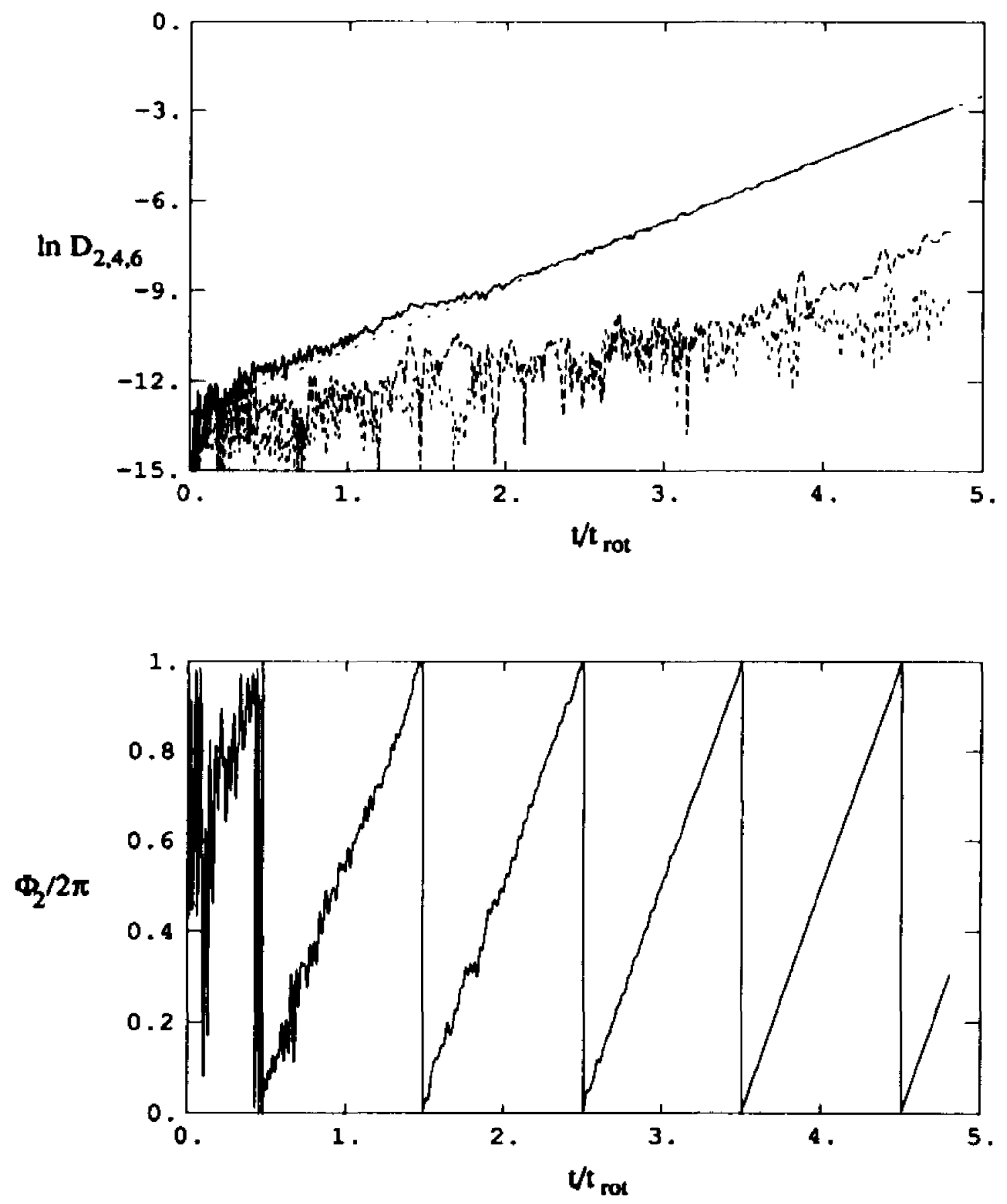

Figure 2.6a: The $m=2, m=4$, and $m=6$ Fourier amplitude behavior, and the $m=2$ phase behavior as a function of time, illustrated as in Figure 2.3a for $M_{d} / M_{c}=\infty$, model \#4. 

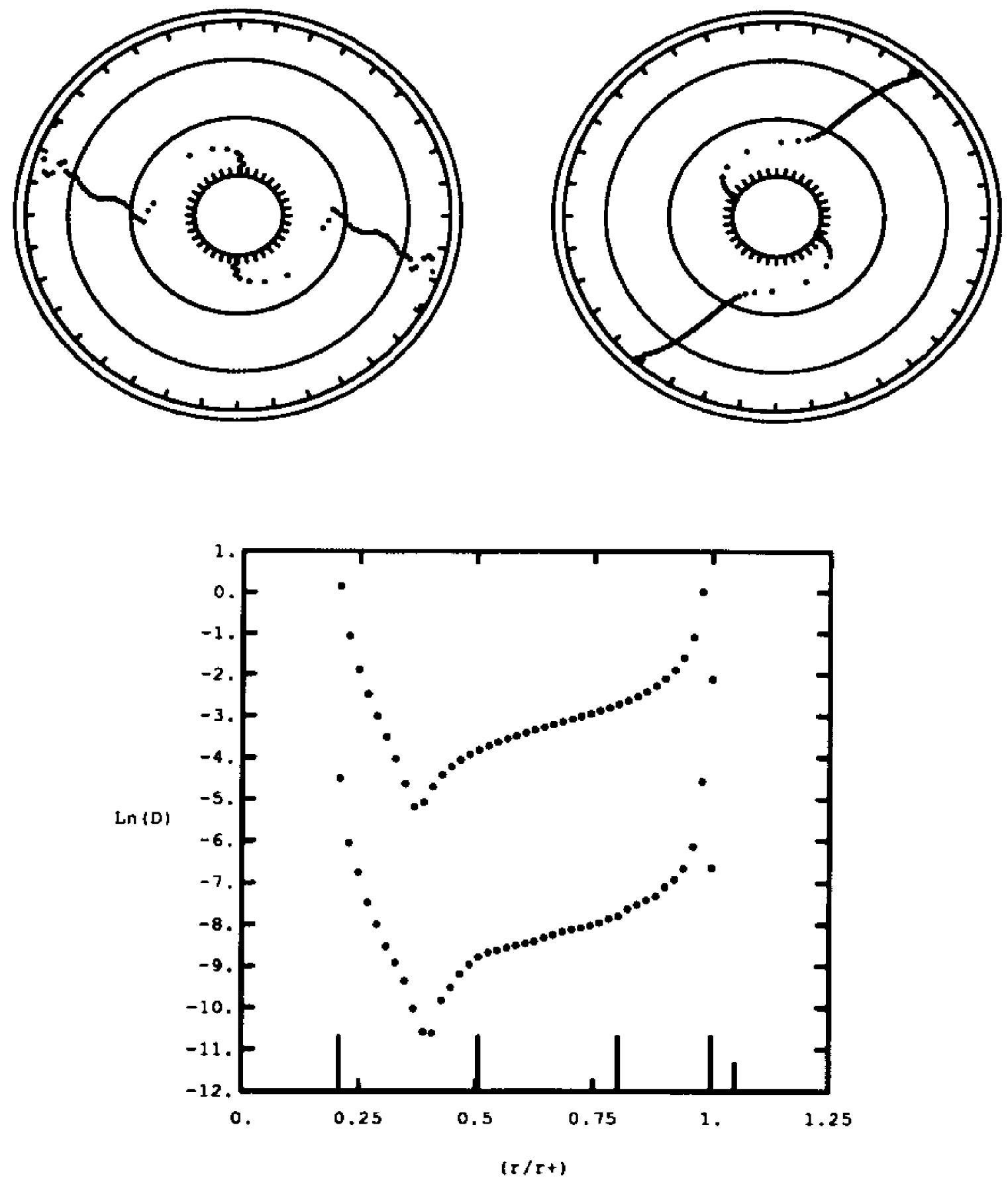

Figure 2.6b: The radial eigenfunction $\left(\phi_{2}-r\right)$ and amplitude as a function of radius $\left(D_{2}-r\right)$ of the $m=2$ Fourier mode illustrated as in Figure 2.3b for $M_{d} / M_{c}=\infty$, model \#4. 

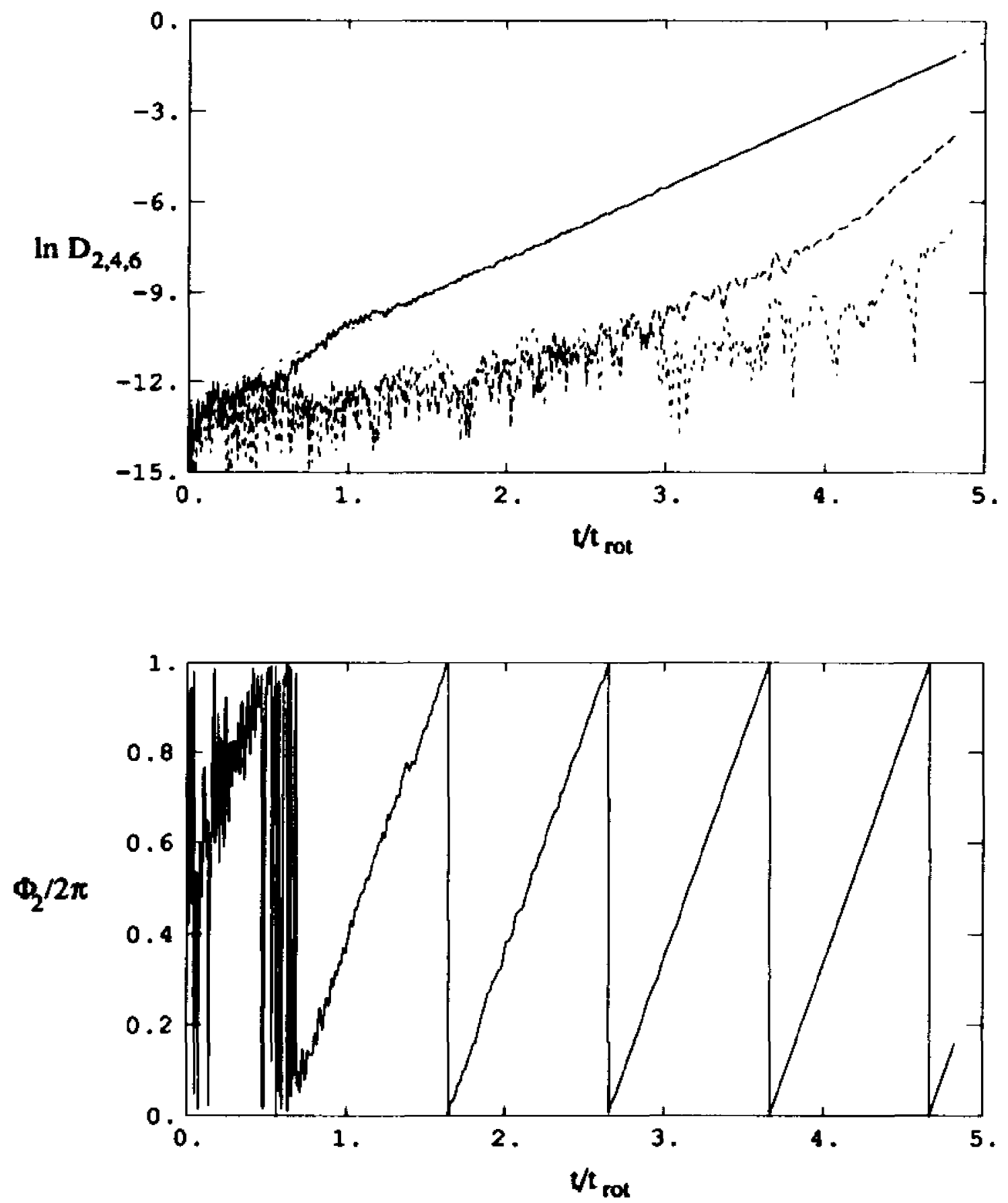

Figure 2.7a: The $m=2, m=4$, and $m=6$ Fourier amplitude behavior, and the $m=2$ phase behavior as a function of time, illustrated as in Figure 2.3a for $M_{d} / M_{c}=\infty$, model \#5. 

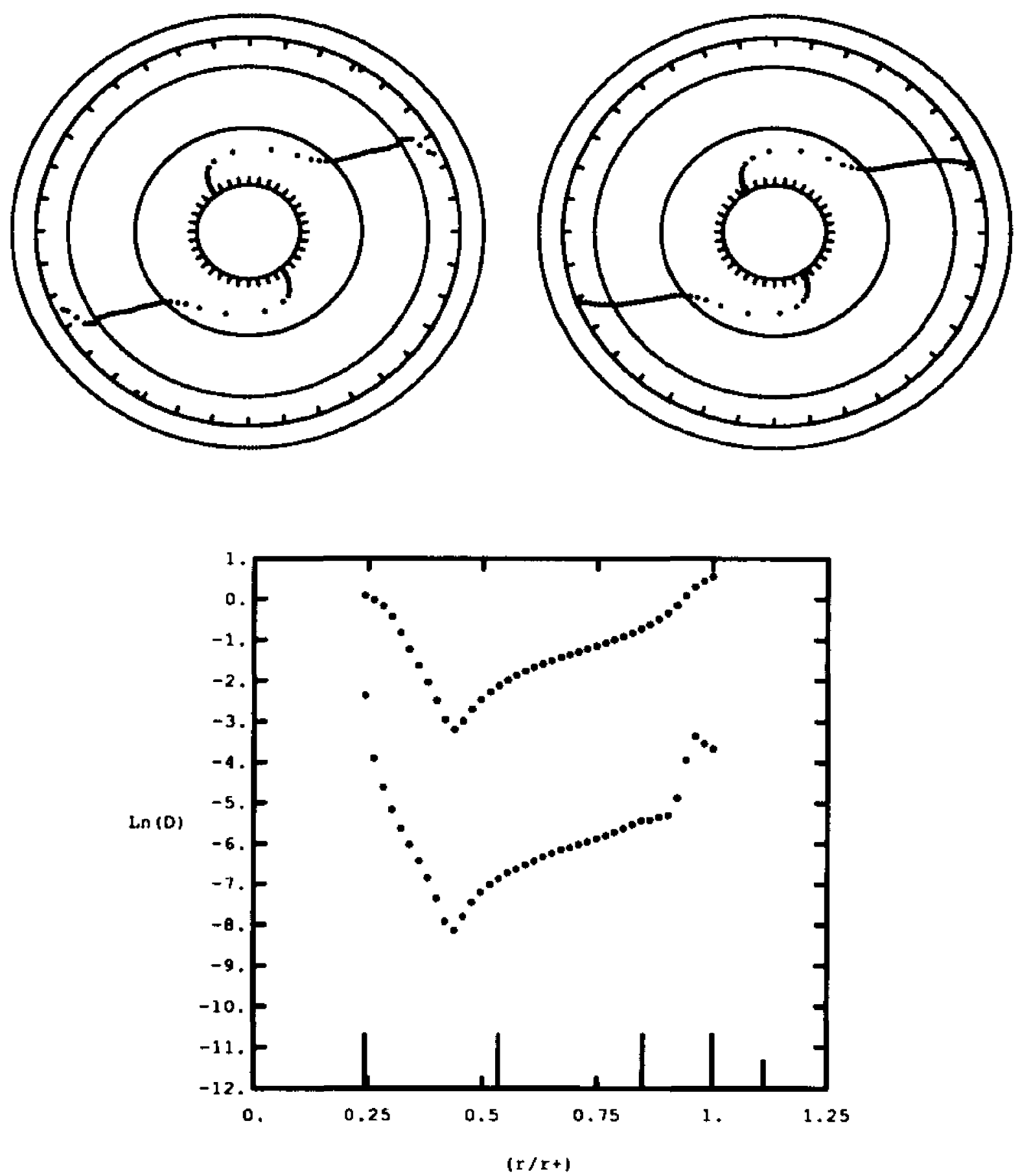

Figure 2.7b: The radial eigenfunction $\left(\phi_{2}-r\right)$ and amplitude as a function of radius $\left(D_{2}-r\right)$ of the $m=2$ Fourier mode illustrated as in Figure 2.3b for $M_{d} / M_{c}=\infty$, model \#5. 

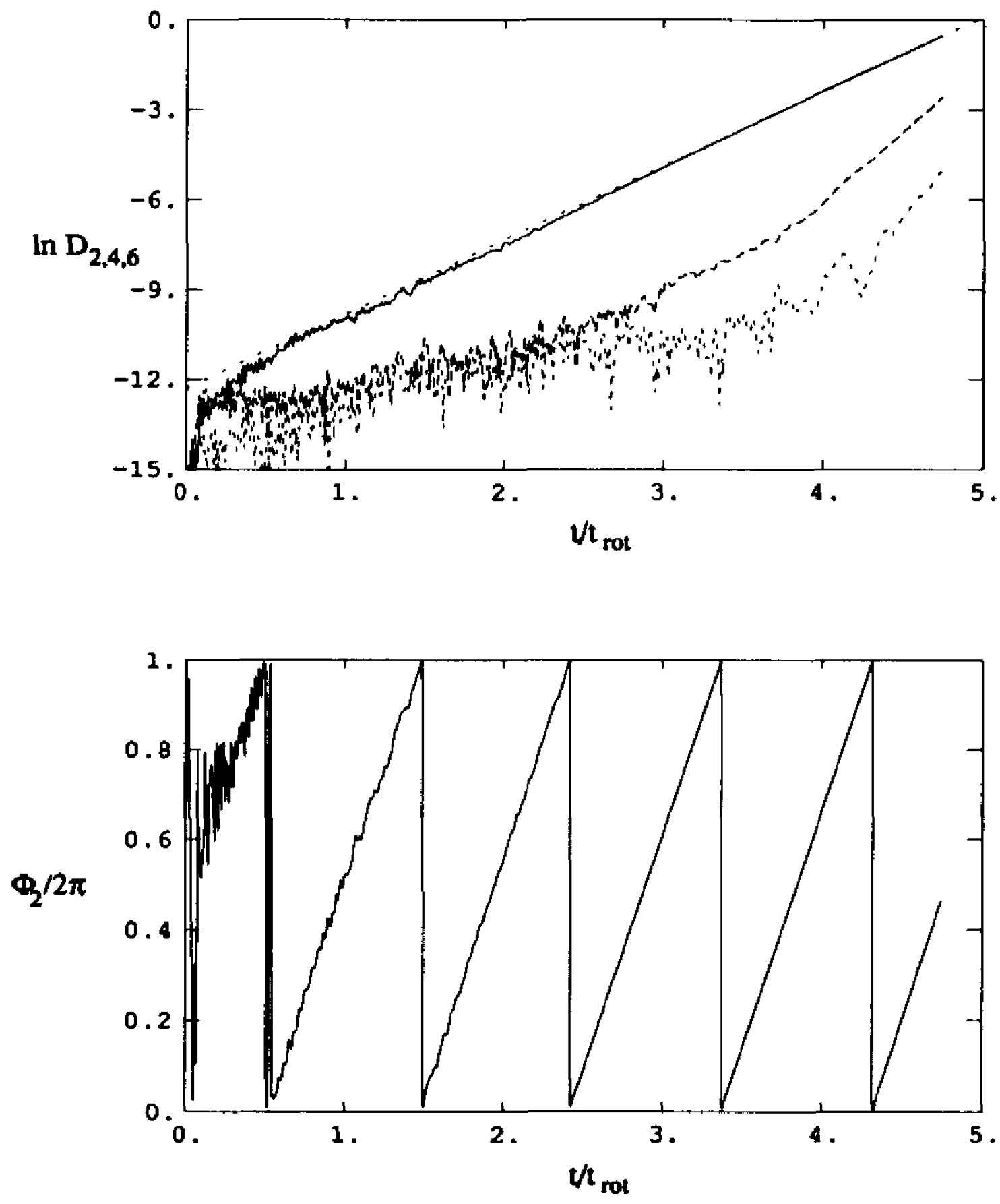

Figure 2.8a: The $m=2, m=4$, and $m=6$ Fourier amplitude behavior, and the $m=2$ phase behavior as a function of time, illustrated as in Figure 2.3a for $M_{d} / M_{c}=\infty$, model $\# 6$. 

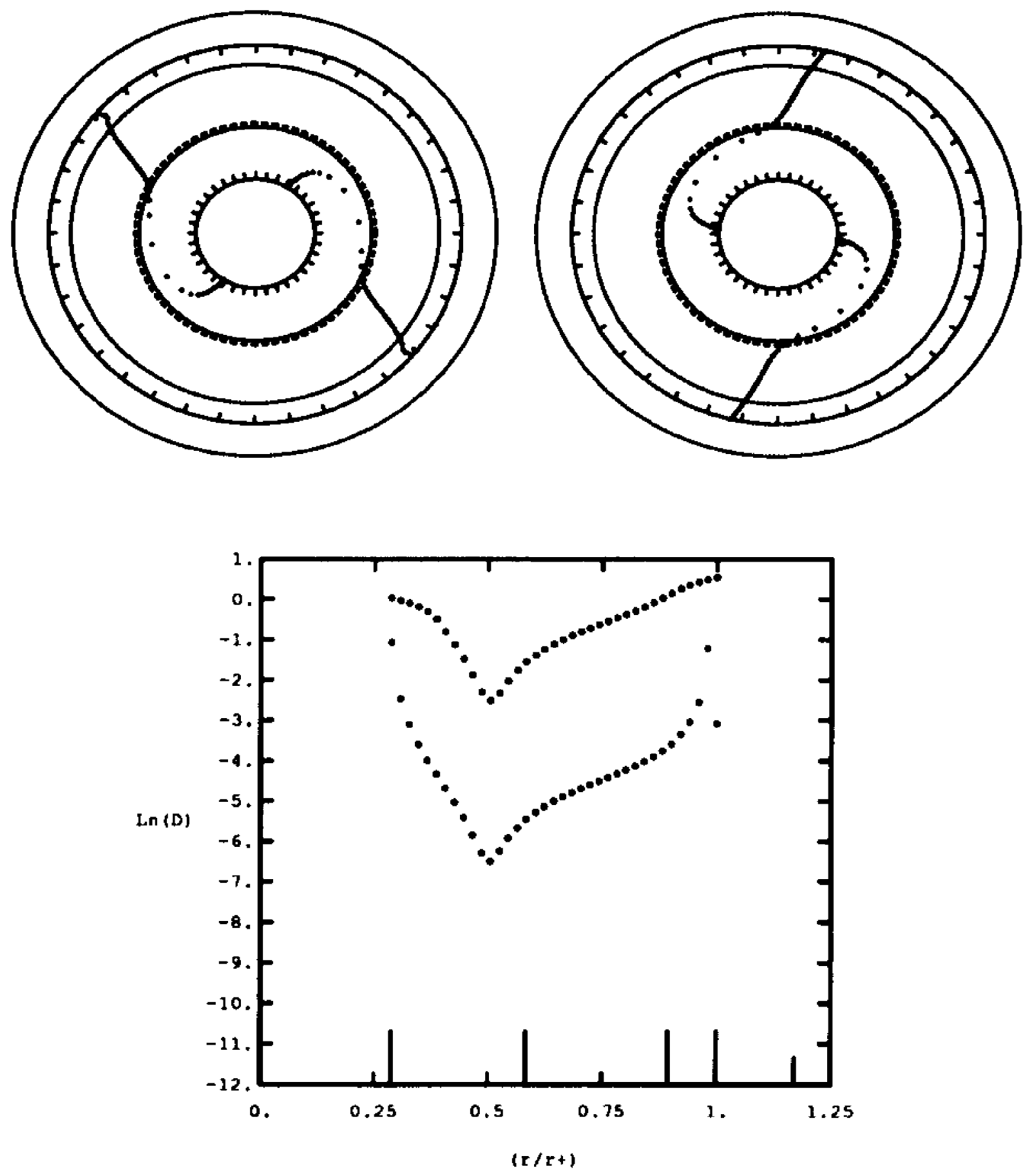

Figure 2.8b: The radial eigenfunction $\left(\phi_{2}-r\right)$ and amplitude as a function of radius $\left(D_{2}-r\right)$ of the $m=2$ Fourier mode illustrated as in Figure 2.3b for $M_{d} / M_{c}=\infty$, model \#6. 

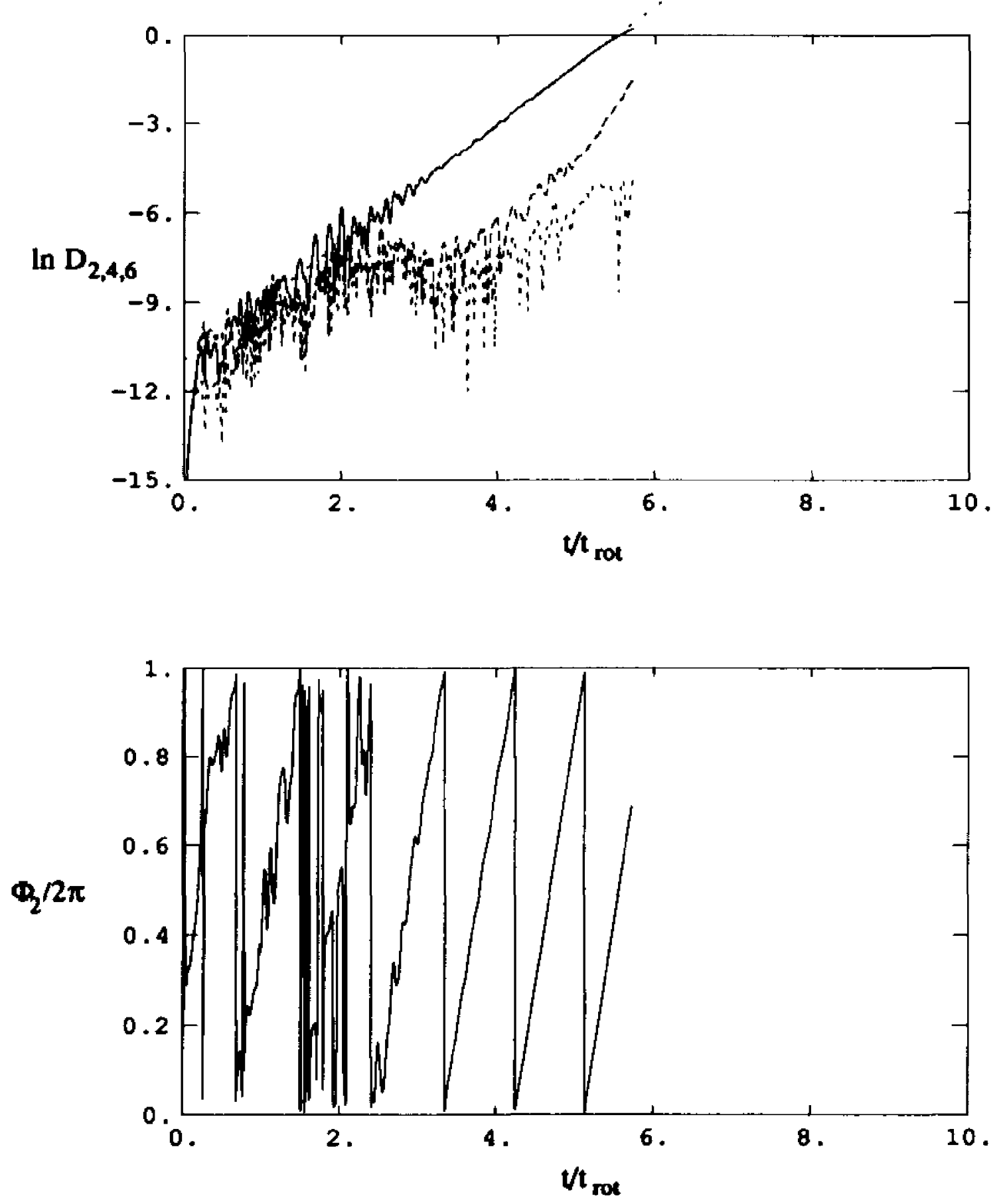

Figure 2.9a: The $m=2, m=4$, and $m=6$ Fourier amplitude behavior, and the $m=2$ phase behavior as a function of time, illustrated as in Figure 2.3a for $M_{d} / M_{c}=\infty$, model \#7. 

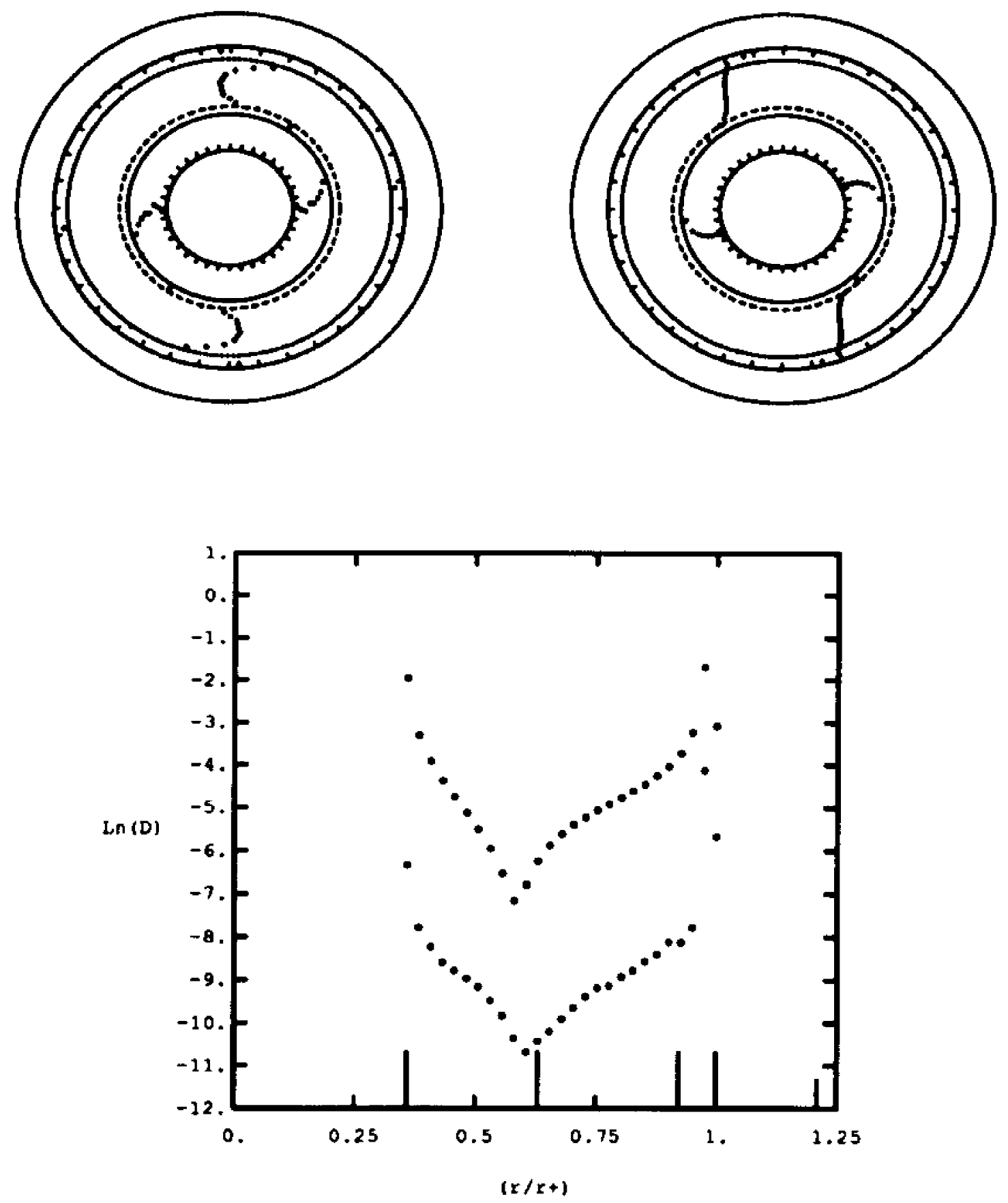

Figure 2.9b: The radial eigenfunction $\left(\phi_{2}-r\right)$ and amplitude as a function of radius $\left(D_{2}-r\right)$ of the $m=2$ Fourier mode illustrated as in Figure 2.3b for $M_{d} / M_{c}=\infty$, model \#7. 

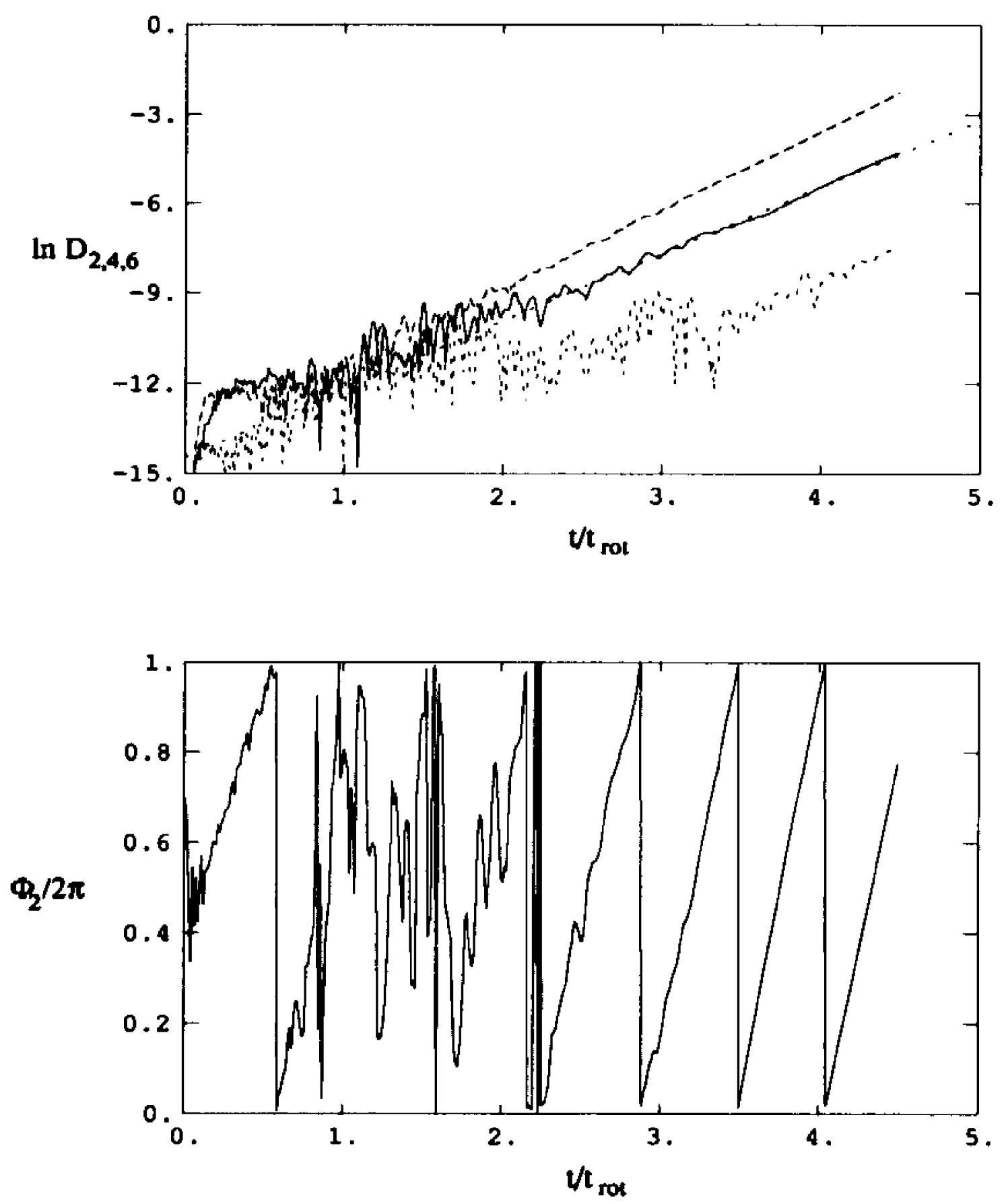

Figure 2.10a: The $m=2, m=4$, and $m=6$ Fourier amplitude behavior, and the $\boldsymbol{m}=\mathbf{2}$ phase behavior as a function of time, illuatrated as in Figure 2.3a for $M_{d} / M_{c}=\infty$, model \#8. 

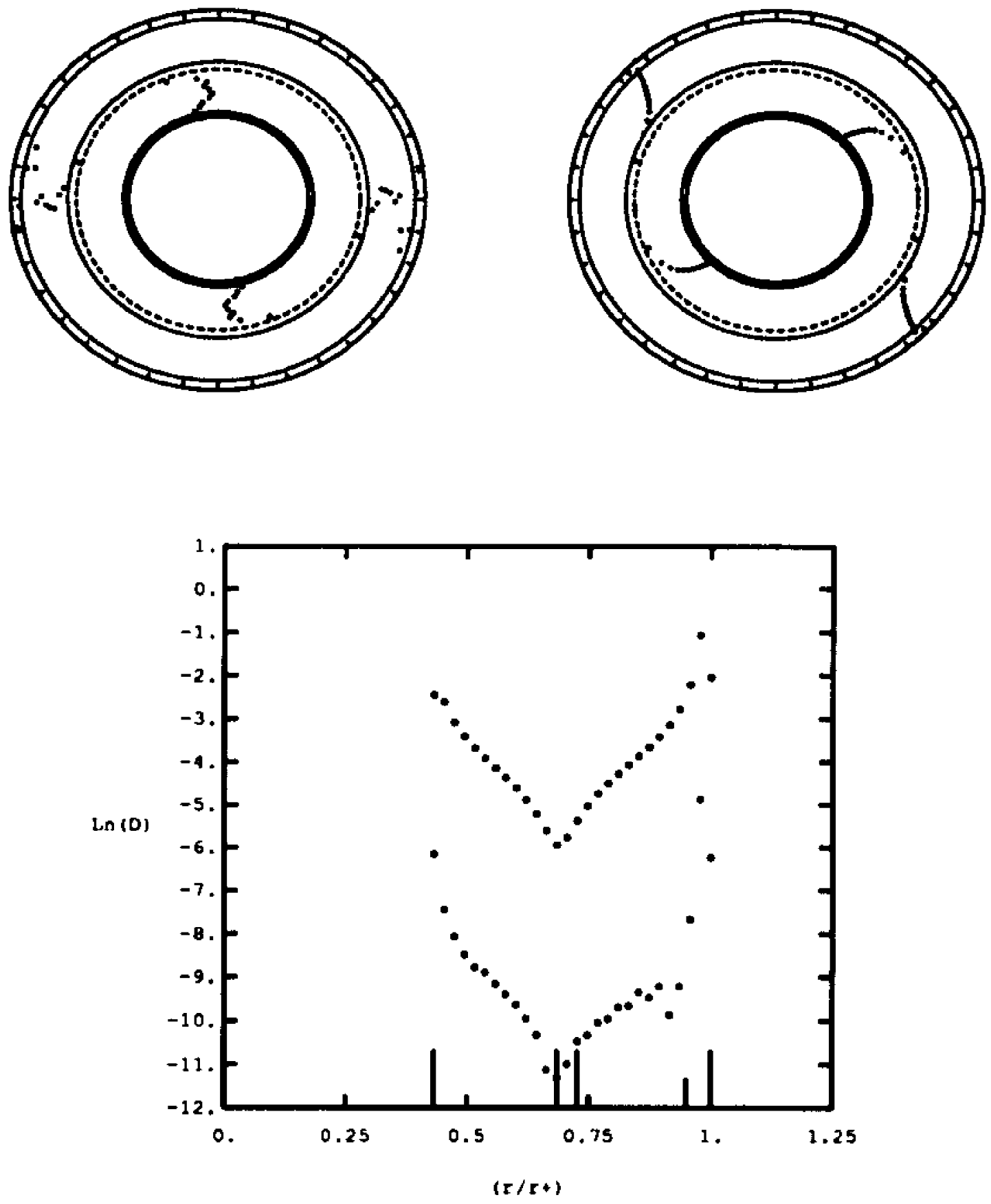

Figure 2.10b: The radial ejgenfunction $\left(\phi_{2}-r\right)$ and amplitude as a function of radius $\left(D_{2}-r\right)$ of the $m=2$ Fourier mode illustrated as in Figure 2.3b for $M_{d} / M_{c}=\infty$, model \#8. 

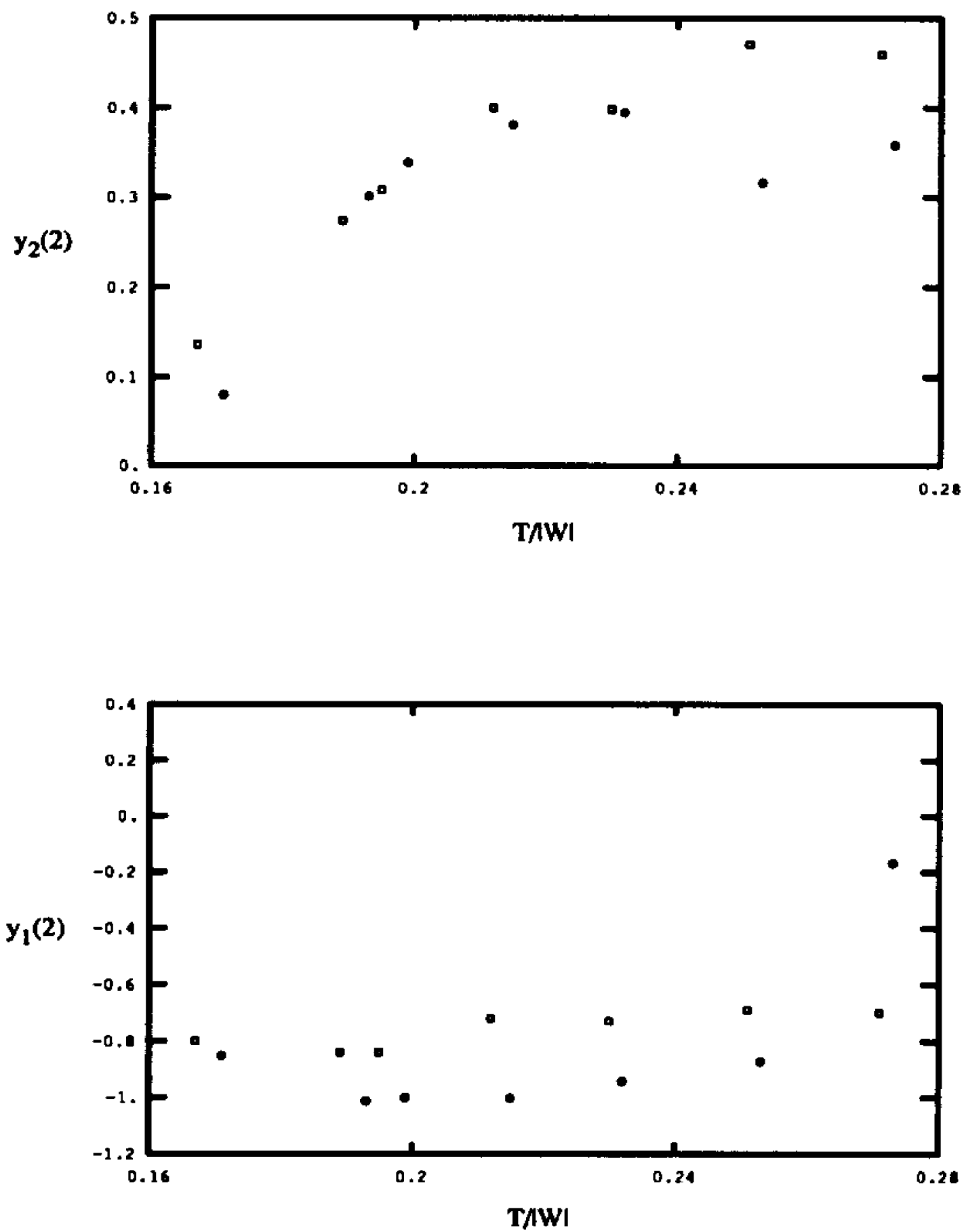

Figure 2.11: $y_{2}(2)$ and $y_{1}(2)$ as functions of $T /|W|$ for $M_{d} / M_{c}=\infty$ models evolved with the TH90 hydrodynamic code (open squares) and with the present, fully second-order code (filled circles). 


\section{CHAPTER 3 \\ SELF-GRAVITATING DISKS IN THE \\ PRESENCE OF AN INERT, CENTRAL POINT MASS}

\subsection{Introduction}

In order to extend our stability analysis to systems other than those that are dominated by a very massive disk, we must consider the effects that the addition of a central object will have on the resulting system. We begin this endeavor by extending the work of the previous chapter in a very straightforward manner, maintaining all the various constraints that were imposed on the original models. Later chapters will be devoted to situations in which these constraints are relaxed.

\subsection{Heavy Disks: Straight forward Evolutions}

In extending the analysis to systems with disk-to-central-object mass ratios closer to unity, we follow precisely the same procedure as outlined in Chapter 2: we generate a series of models, evolve them one at a time while enforcing $\pi+$ symmetry, and plot the $y_{2}(2)$ parameter versus $T /|W|$ in order to determine the critically unstable value of $T /|W|$ for a particular mass ratio. In many ways, we have become experimentalists. We have a tested apparatus - our hydrodynamic code, along with the accompanying initial model generating code. We then explore parameter space, examining certain potentially interesting behaviors and reporting what observations we have made. More often than not, we do not attempt to explain the 'why' of what has been observed. Usually, that question of 'why' takes us beyond the scope of the present study. For a particular mass ratio, we want to know at what value of $T /|W|$ an accretion disk becomes unstable - not why it is unstable, or what drives 
the instability. We leave those questions to be answered later - perhaps, by others in the field - and content ourselves with reporting an extensive set of methodical 'observations'.

In the preceding chapter, several different rotation laws were used in the selected group of initial models. In this chapter, we restrict ourselves to one rotation law, that deriving from uniform specific angular momentum $(q=2.0)$. Again, note that, according to Eq. (2.18), this particular choice of rotation law forces the inner and outer Lindblad resonances to lie at the corotation radius for all models evolved. An additional constraint is placed on the equation of state, restricting the models to one polytropic constant, $n=1.5$. In this way, the only parameters that remain variable in generating the initial models become the size (radial extent) of the disk, and the mass ratio currently being examined. In later work, other equations of state and rotation laws may be considered but, due to the computationally intensive nature of a single evolution, these additional variations are not pursued at this time.

Systems having three different mass ratios are examined here: $M_{d} / M_{c}=5.0$, 1.0, and 0.2. For each mass ratio, 6 models ( 7 for mass ratio 0.2 ) are evolved using the second-order-accurate hydrodynamic code. Note that the enforced " $\pi$ symmetry" constrains the central object to remain at the center of the grid at all times. Data describing all 19 initial models are given in Tables 3.1, 3.3, and 3.5. In an attempt to summarize the principal results of the 19 dynamical evolutions, the measured values of $y_{1}(2)$ and $y_{2}(2)$ have been tabulated in Tables 3.2, 3.4, and 3.6, and displayed in Figures 3.1, 3.8, 3.15, and (the overall summary) 3.23. 


\subsubsection{Systems with $M_{d} / M_{c}=5.0$}

It is evident from the results displayed in Table 3.2 and Figure 3.1 that the critically unstable model is that at $T /|W|=0.238$ (model \#2). This model is very slowly growing, with a growth rate parameter $y_{2}(2)=0.027$. Model $\# 1$, with $T /|W|=0.217$, is also slowly growing, with a growth rate not significantly lower than that of model \#2. We are faced with the question as to whether or not models with $T /|W|$ less than that of model \#2 are stable because of this slight change in growth rates (i.e., does the growth rate asymptotically approach zero as $T /|W|$ declines?). This is a difficult question to answer, as models with such a low growth rate have not been evolved before (eg., the lowest growth rate reported in Chapter 2 was $\left.y_{2}(2) \sim 0.08\right)$. One might argue that model \# 1 actually lies somewhat below the critically unstable $T /|W|$ value by examining the $D_{2}-t$ plot in figure $3.2 \mathrm{a}$. It seems that the mode grew with a modest growth rate for 5 rotation periods, then the growth rate declined. This may be indicative of the establishment of a new equilibrium state. Additionally, the $\phi_{2}-t$ plot does not indicate that the mode possesses a coherent pattern speed. This behavior is not present in model \#2, in which the instability grows steadily over the entire evolution, establishing a coherent pattern speed. Both models \#1 and \#2 show a definite phase versus radius eigenfunction behavior emerging (see Figures $3.2 \mathrm{~b}$ and $3.3 \mathrm{~b}$ ). Thus, we conclude that model \#1 lies just below the threshold of instability, and as such, model \#2 is identified as the critically unstable model for this mass ratio.

Model \#6, the first model actually evolved in the hydrodynamic code for this mass ratio, proved to be violently unstable. The growth rate, despite the rapidity of the evolution, is well defined. (In this case, the model evolved quite rapidly, reaching the nonlinear growth regime and fragmenting after only 2000 time-steps.) 
The $y_{1}(2)$ parameter could be determined only through one phase cycle, so it may not be an accurate measure of the pattern speed. Note also that the $m=4$ mode grows more rapidly than the $m=2$ mode, as we saw in the highest $T /|W|$ models of Chapter 2.

The other models, \#3 through \#5, show the same basic behavior as the mass ratio $\infty$ models - well established growth rate and pattern speed for an $\boldsymbol{m}=2$ distortion, with a slight increase in the amplitude of the higher order modes as we go to higher $T /|W|$. In the $\phi_{2}-r$ plots, the phase orientation of the distortion undergoes a $\pi / 2$ phase shift just inside the pressure maximum $r_{0}$, over relatively few radial grid zones ( 1 to 3 zones). As evidenced in the $D_{2}-r$ plots, this phase shift in $\phi_{2}$ occurs at the same radius in the torus where there is a minimum in the amplitude of the distortion.

In models \#3 through \#5, the corotation point (and, hence, the inner and outer Lindblad resonances) appears to have no obvious effect on the latter part of the evolutions. There is an indication that the corotation point has some effect in the very early stages of the evolution, characterized by the $\phi_{2}-r$ plot bending at these points. From these 7 model evolutions we have learned little new except the critical value of $T /|W|$ at which the $m=2$ mode first becomes unstable in accretion disk systems with $M_{d} / M_{c}=5$. 
Table 3.1

Initial Model Data for $M_{d} / M_{c}=\mathbf{5 . 0}$

\begin{tabular}{cccc}
\hline \hline Model \# & $r_{-} / r_{+}$ & $r_{0} / r_{+}$ & $T /|W|$ \\
1 & 0.166 & 0.398 & 0.217 \\
2 & 0.203 & 0.453 & 0.238 \\
3 & 0.240 & 0.509 & 0.256 \\
4 & 0.333 & 0.601 & 0.292 \\
5 & 0.425 & 0.675 & 0.317 \\
6 & 0.703 & 0.842 & 0.363 \\
\hline
\end{tabular}


Table 3.2

$\mathrm{m}=2$ Eigenmode Character, $M_{d} / M_{c}=5.0$

\begin{tabular}{cccccc}
\hline \hline Model & $y_{1}$ & $y_{2}$ & $\Omega_{p} / \Omega_{0}$ & $r_{c r} / r_{0}$ & $r_{c r} / r_{+}$ \\
1 & - & $(0.023)$ & - & - & - \\
2 & -0.93 & 0.027 & 0.53 & 1.36 & 0.62 \\
3 & -0.89 & 0.211 & 0.55 & 1.34 & 0.68 \\
4 & -0.96 & 0.486 & 0.51 & 1.38 & 0.83 \\
5 & -0.97 & 0.554 & 0.51 & 1.39 & 0.94 \\
$6^{\dagger}$ & +0.38 & 1.721 & 1.19 & 0.91 & 0.77 \\
\hline
\end{tabular}

Dominated by $m=4$. 

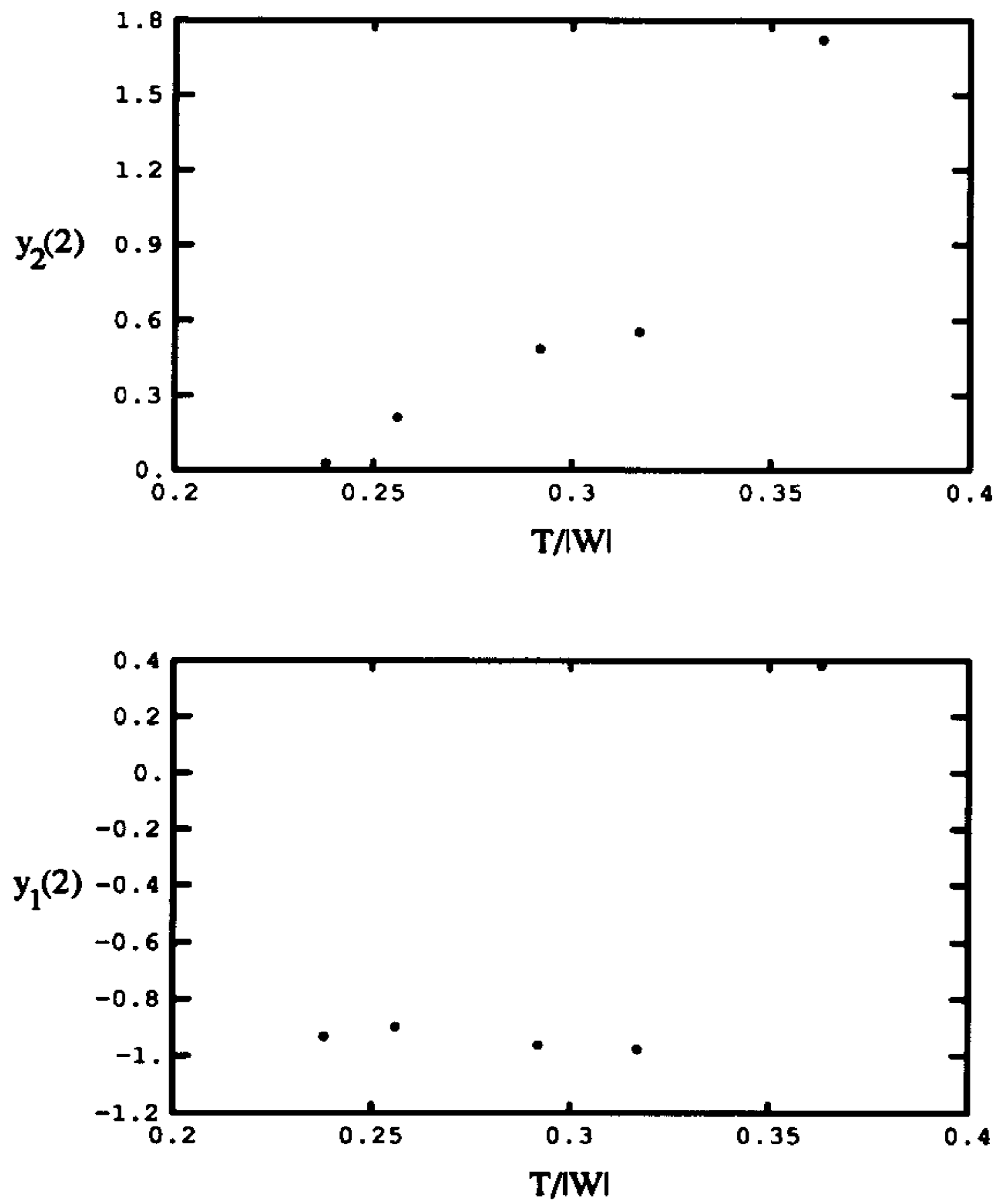

Figure 3.1: $y_{2}(2)$ and $y_{1}(2)$ as functions of $T /|W|$ for $M_{d} / M_{c}=5.0$ models. 

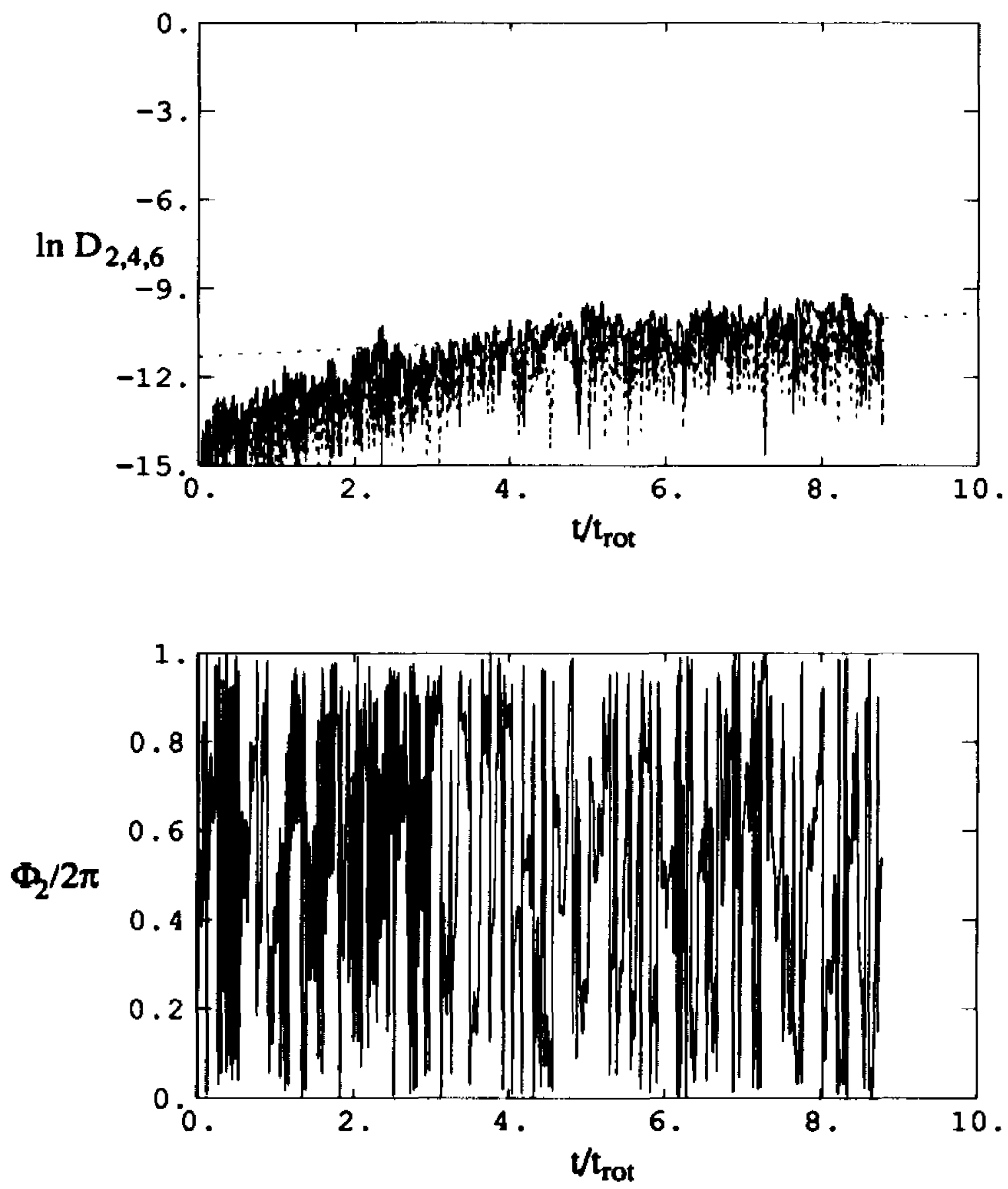

Figure 3.2a: The $m=2, m=4$, and $m=6$ Fourier amplitude behavior, and the $m=2$ phase behavior as a function of time, illustrated as in Figure 2.3a for $M_{d} / M_{c}=5.0$, model \#1. 

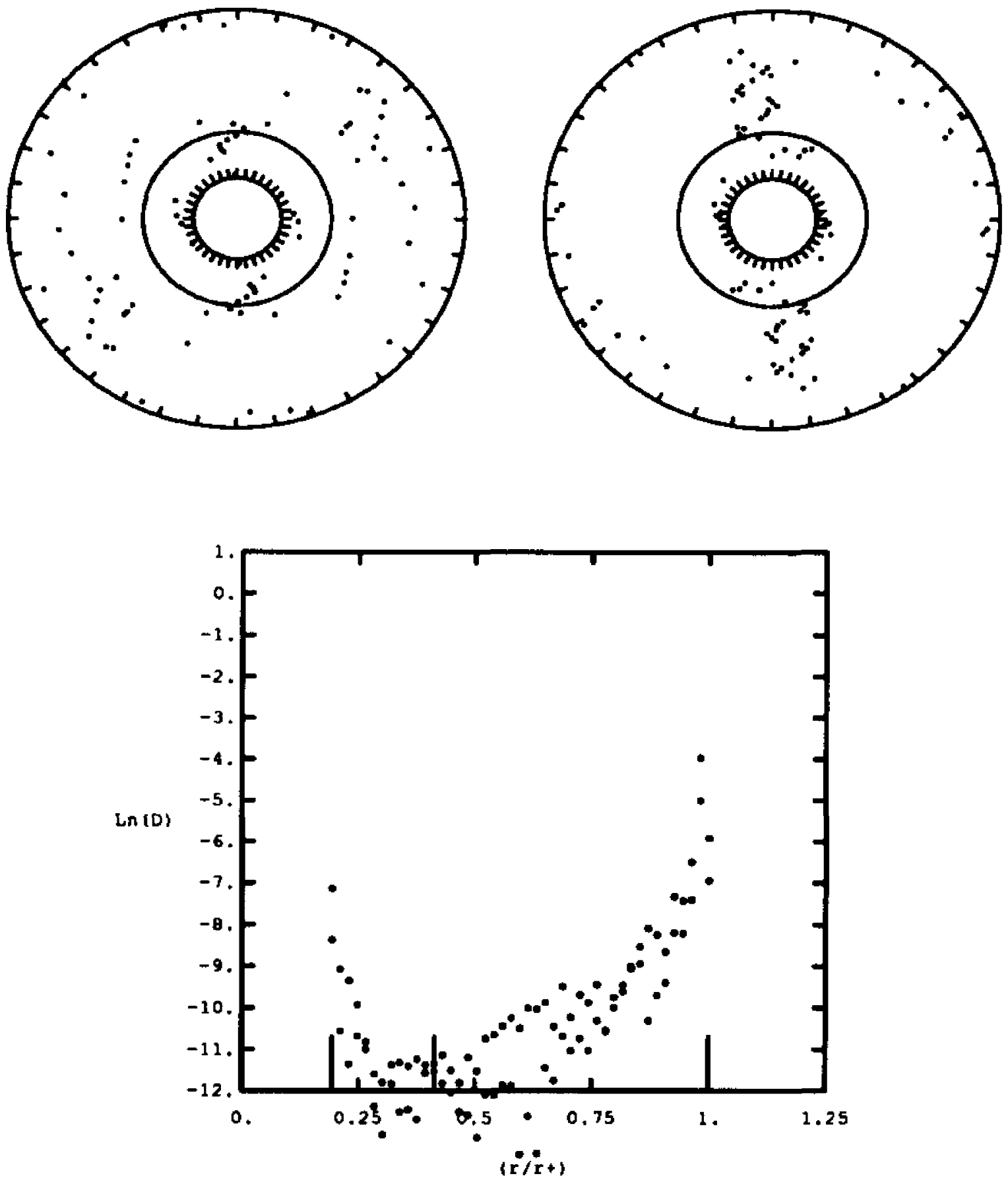

Figure 3.2b: The radial eigenfunction $\left(\phi_{2}-r\right)$ and amplitude as a function of radius $\left(D_{2}-r\right)$ of the $m=2$ Fourier mode illustrated as in Figure $2.3 \mathrm{~b}$ for $M_{d} / M_{c}=5.0$, model $\# 1$. 

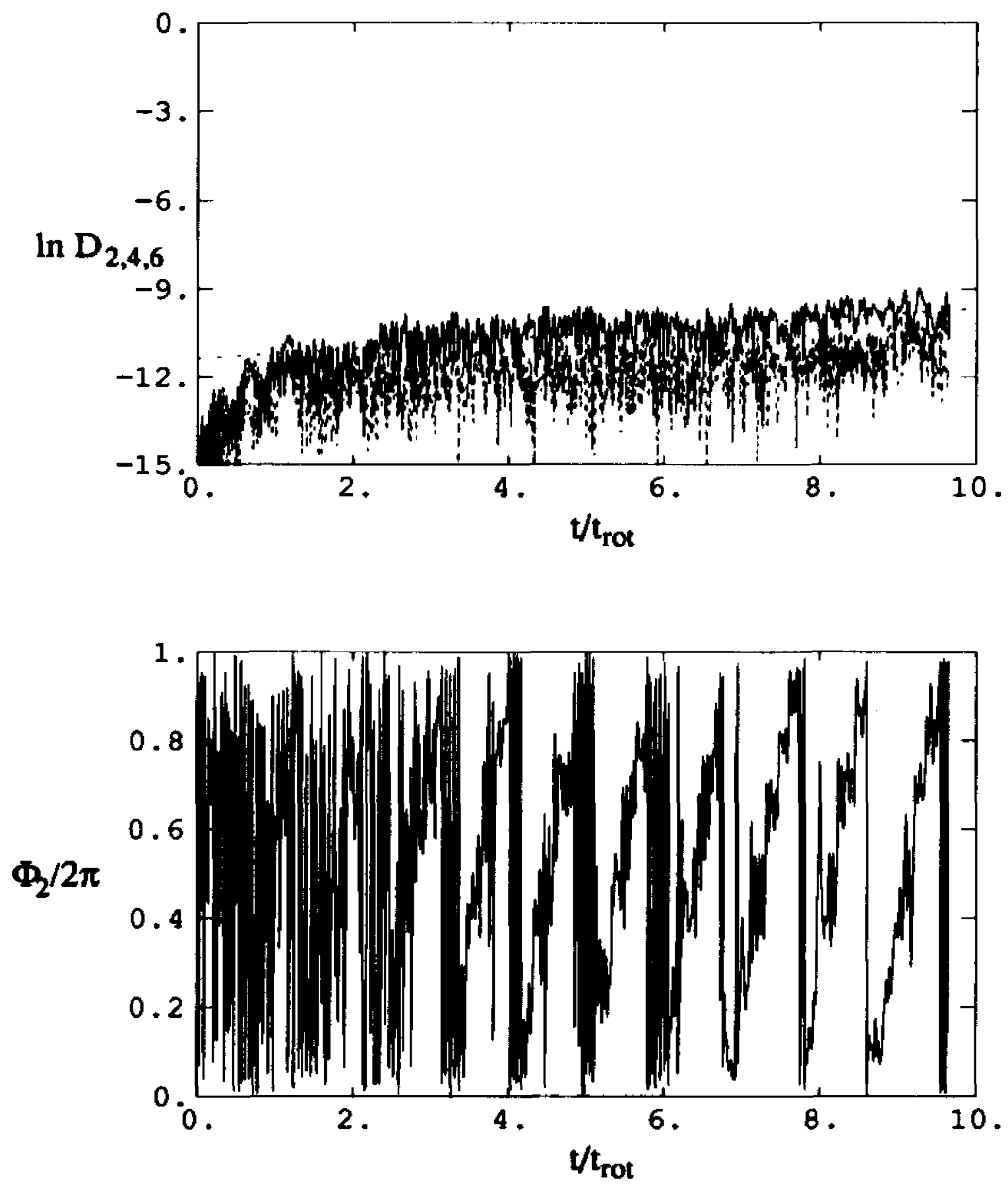

Figure 3.3a: The $m=2, m=4$, and $m=6$ Fourier amplitude behavior, and the $m=2$ phase behavior as a function of time, illustrated as in Figure $2.3 \mathrm{a}$ for $M_{d} / M_{c}=5.0$, model \#2. 

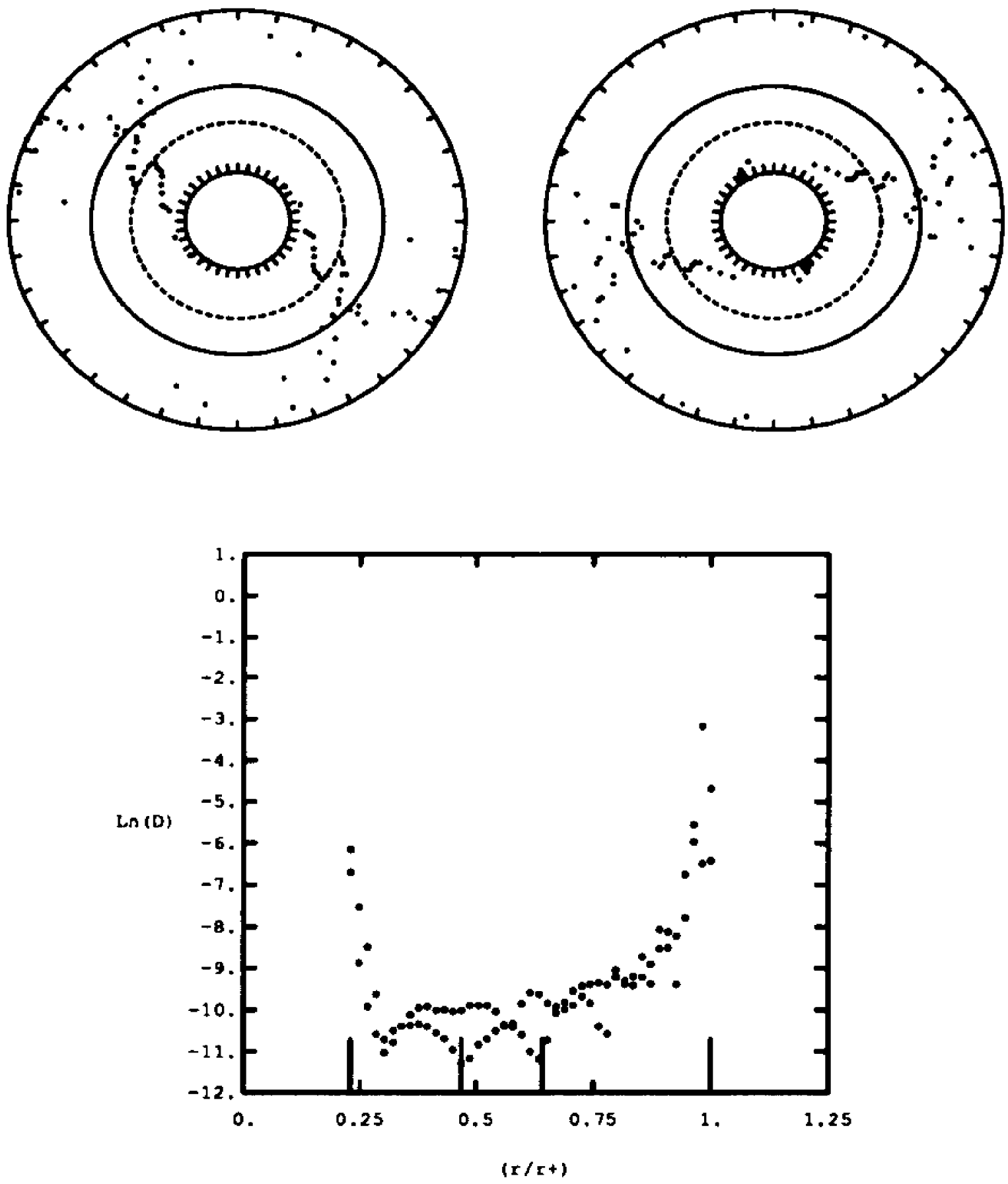

Figure 3.3b: The radial eigenfunction $\left(\phi_{2}-r\right)$ and amplitude as a function of radius $\left(D_{2}-r\right)$ of the $m=2$ Fourier mode illustrated as in Figure $2.3 \mathrm{~b}$ for $M_{d} / M_{c}=5.0$, model \#2. 

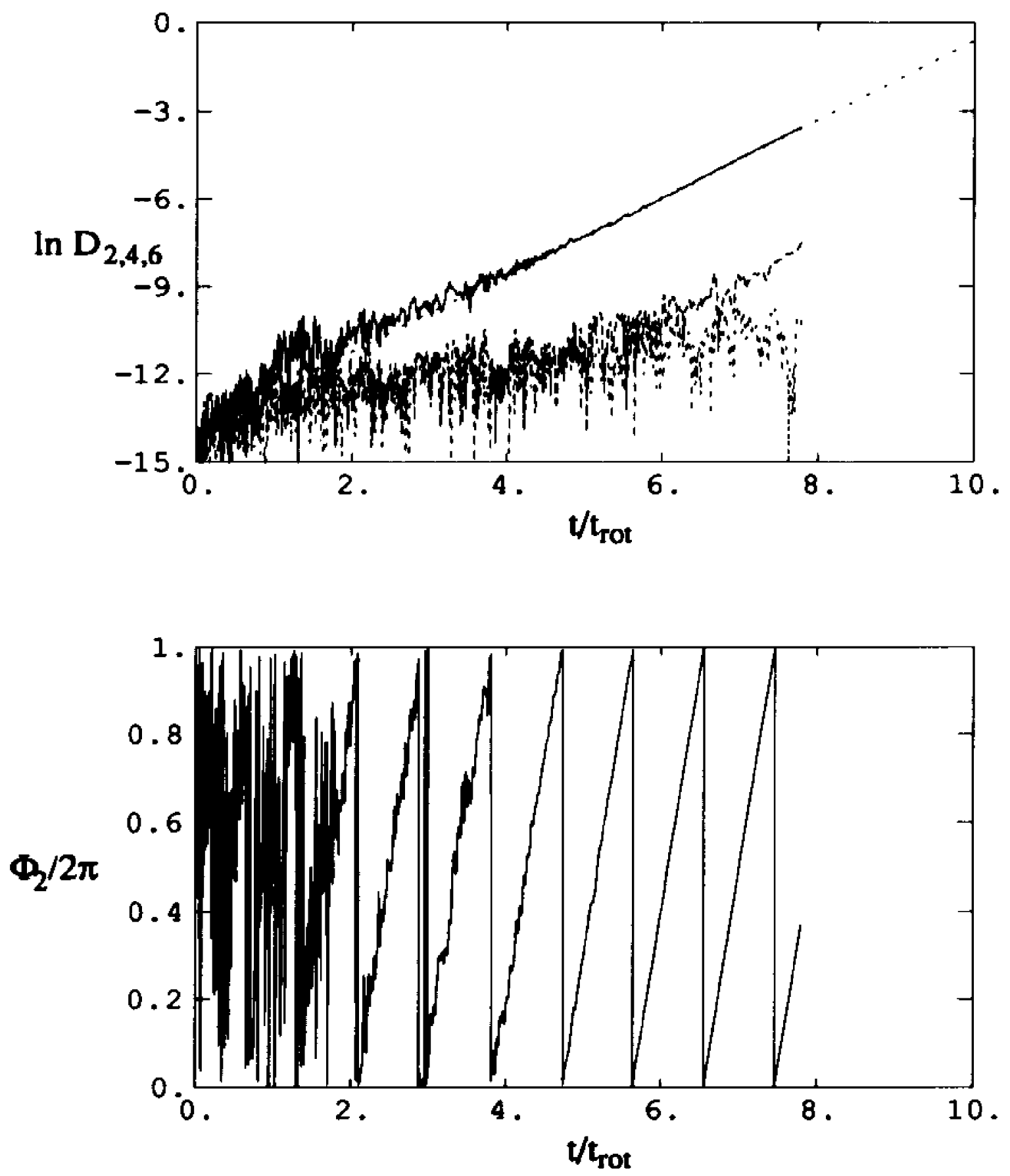

Figure 3.4a: The $m=2, m=4$, and $m=6$ Fourier amplitude behavior, and the $m=2$ phase behavior as a function of time, illustrated as in Figure 2.3a for $M_{d} / M_{c}=5.0$, model \#3. 

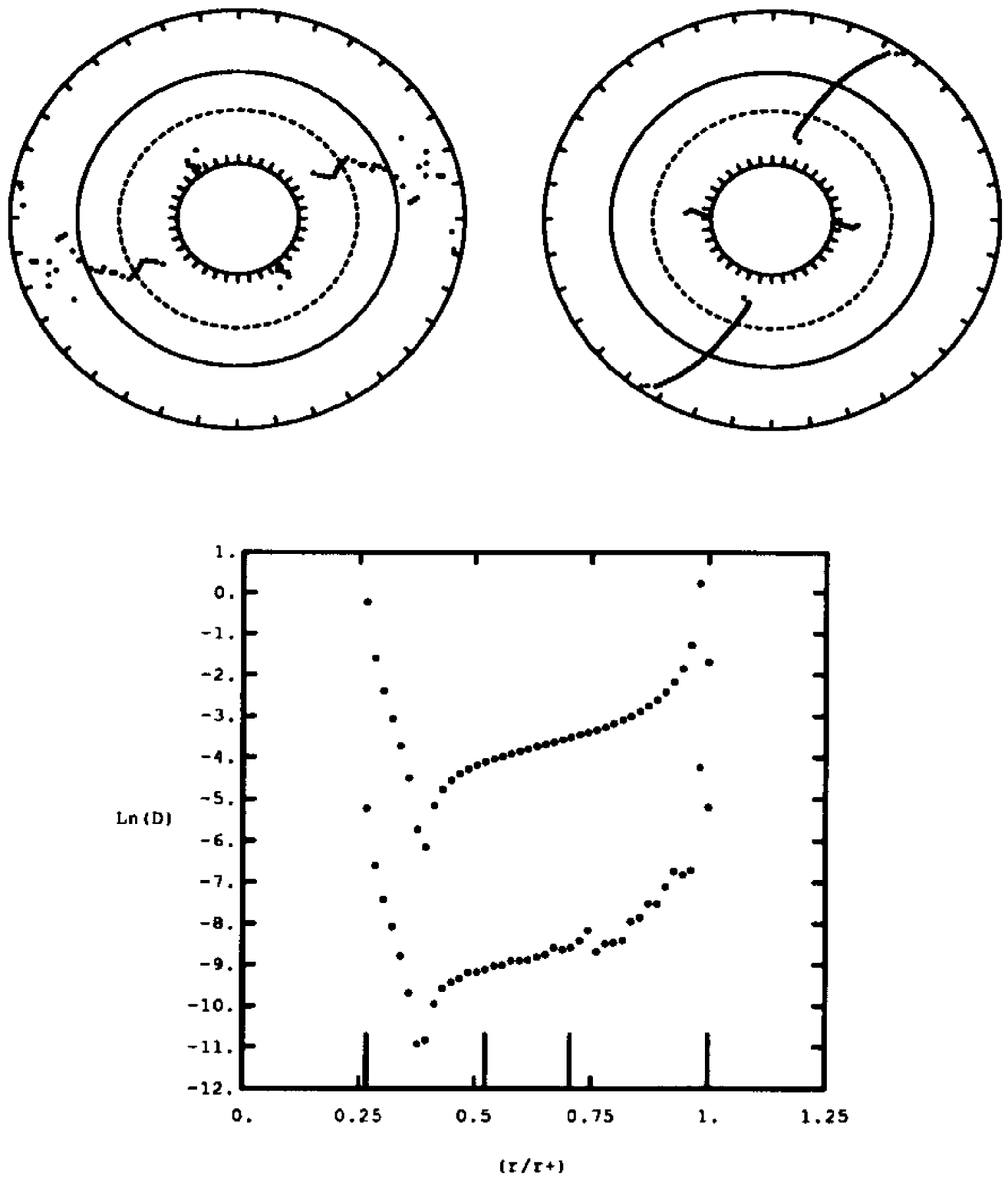

Figure 3.4b: The radial eigenfunction $\left(\phi_{2}-r\right)$ and amplitude as a function of radius $\left(D_{2}-r\right)$ of the $m=2$ Fourier mode illustrated as in Figure 2.3b for $M_{d} / M_{c}=5.0$, model \#3. 

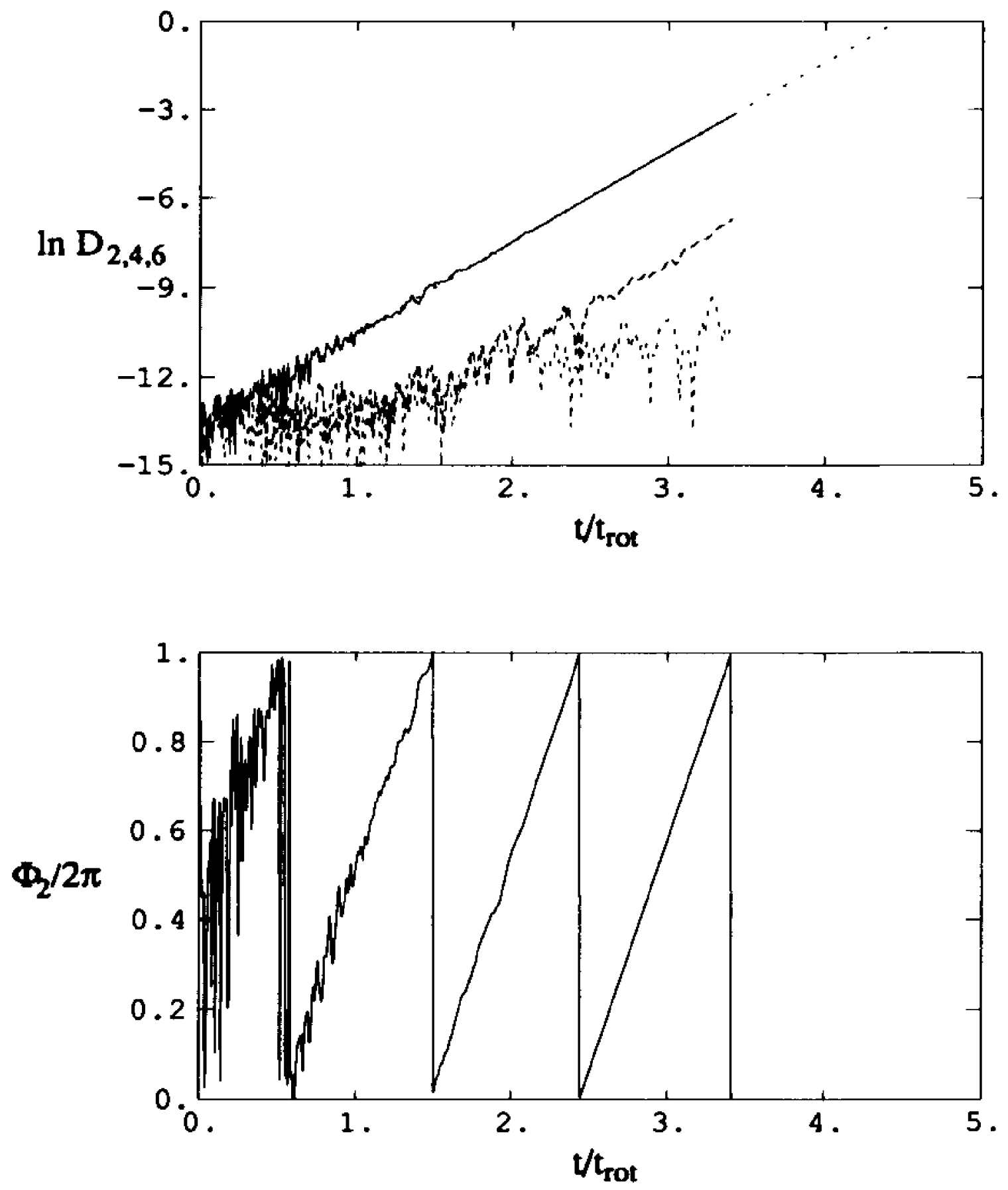

Figure 3.5a: The $m=2, m=4$, and $m=6$ Fourier amplitude behavior, and the $m=2$ phase behavior as a function of time, illustrated as in Figure $2.3 \mathrm{a}$ for $M_{t} / M_{c}=5.0$, model \#4. 

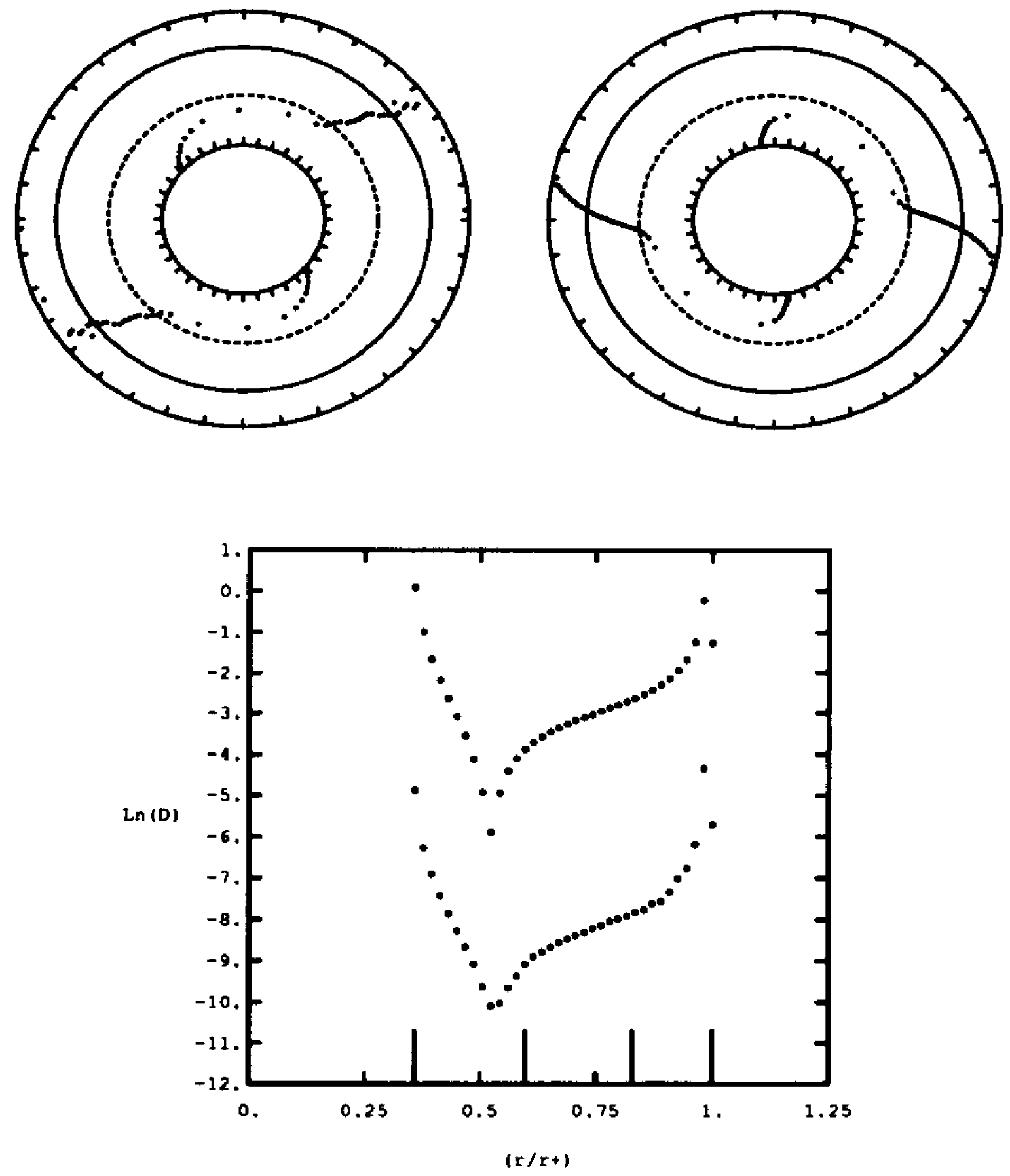

Figure 3.6b: The radial eigenfunction $\left(\phi_{2}-r\right)$ and amplitude as a function of radius $\left(D_{2}-r\right)$ of the $m=2$ Fourier mode illustrated as in Figure 2.3b for $M_{d} / M_{c}=5.0$, model \#4. 

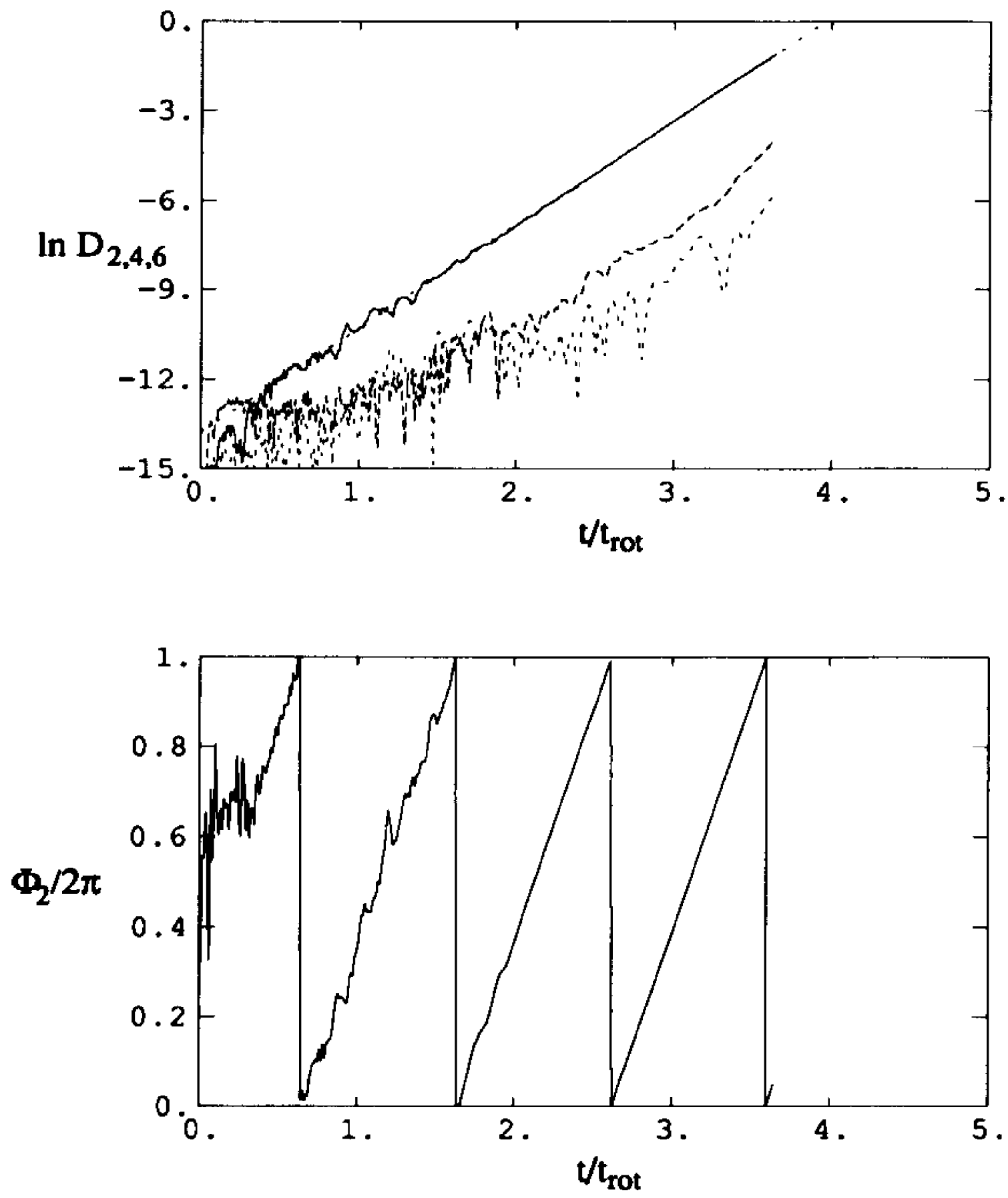

Figure 3.6a: The $m=2, m=4$, and $m=6$ Fourier amplitude behavior, and the $m=2$ phase behavior as a function of time, illustrated as in Figure 2.3a for $M_{d} / M_{c}=5.0$, model \#5. 

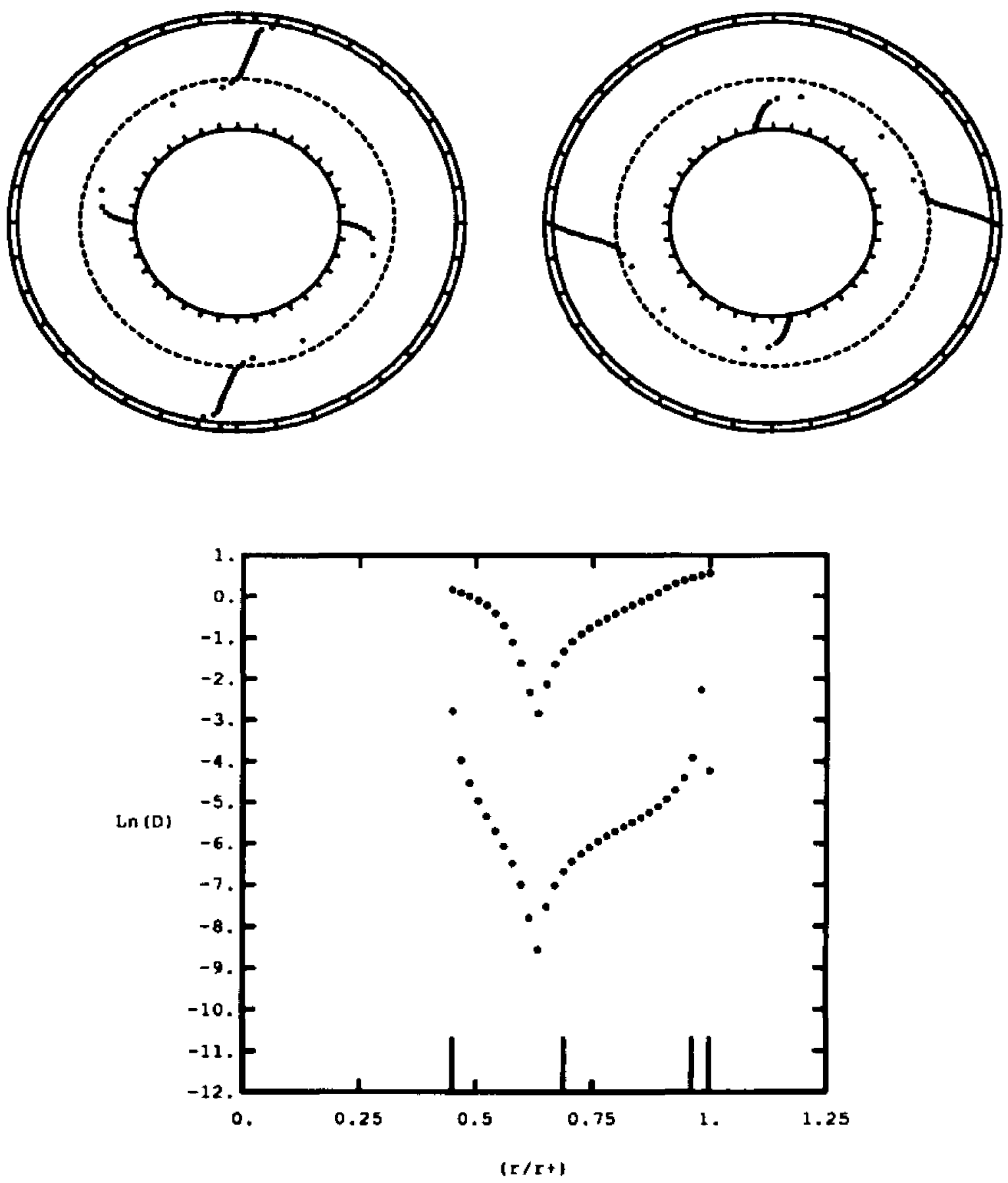

Figure 3.6b: The radial eigenfunction $\left(\phi_{2}-r\right)$ and amplitude as a function of radius $\left(D_{2}-r\right)$ of the $m=2$ Fourier mode illustrated as in Figure $2.3 \mathrm{~b}$ for $M_{d} / M_{\mathrm{c}}=5.0$, model \#5. 

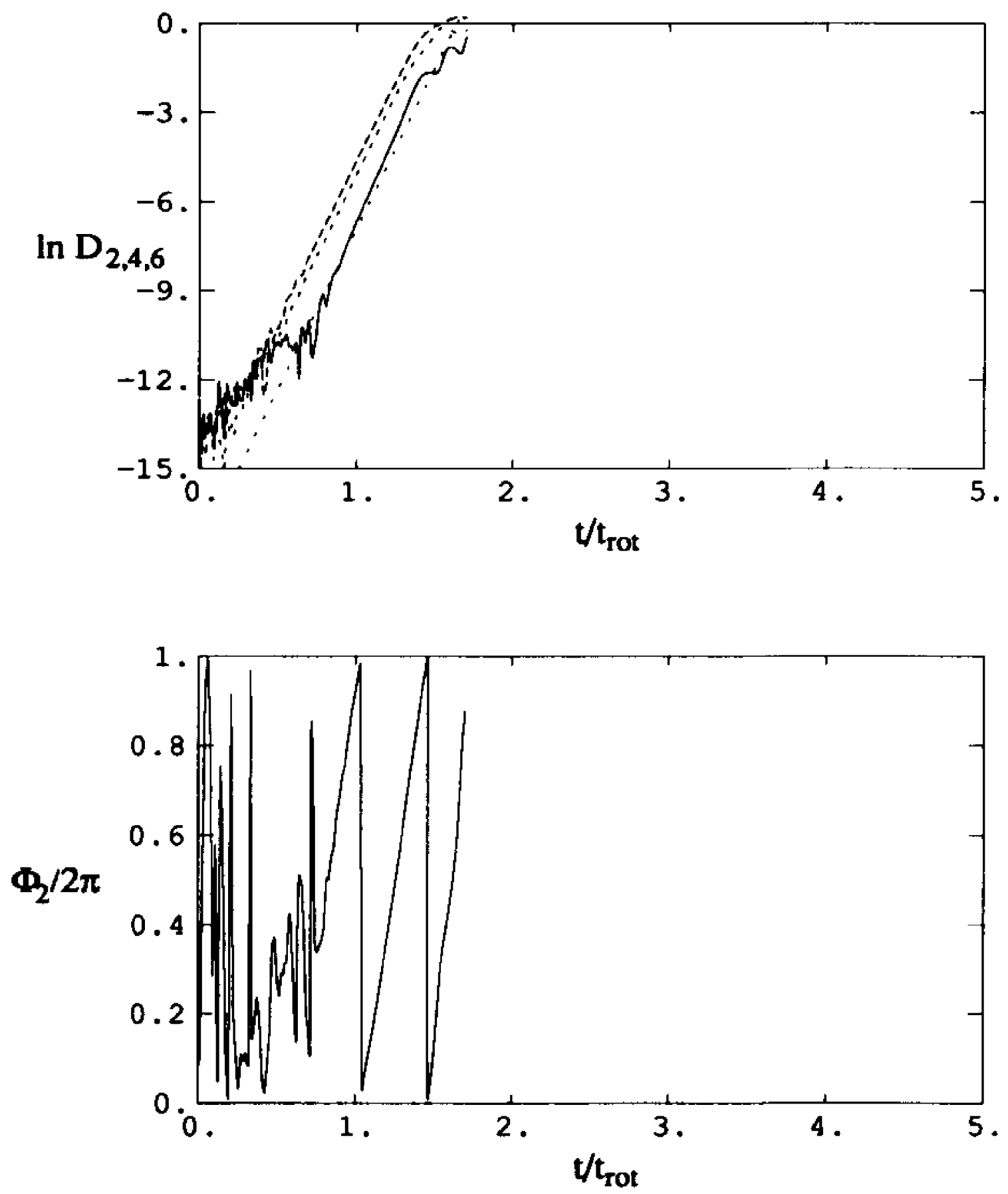

Figure 3.7a: The $m=2, m=4$, and $m=6$ Fourier amplitude behavior, and the $m=2$ phase behavior as a function of time, illustrated as in Figure 2.3a for $M_{d} / M_{c}=5.0$, model \#6. 

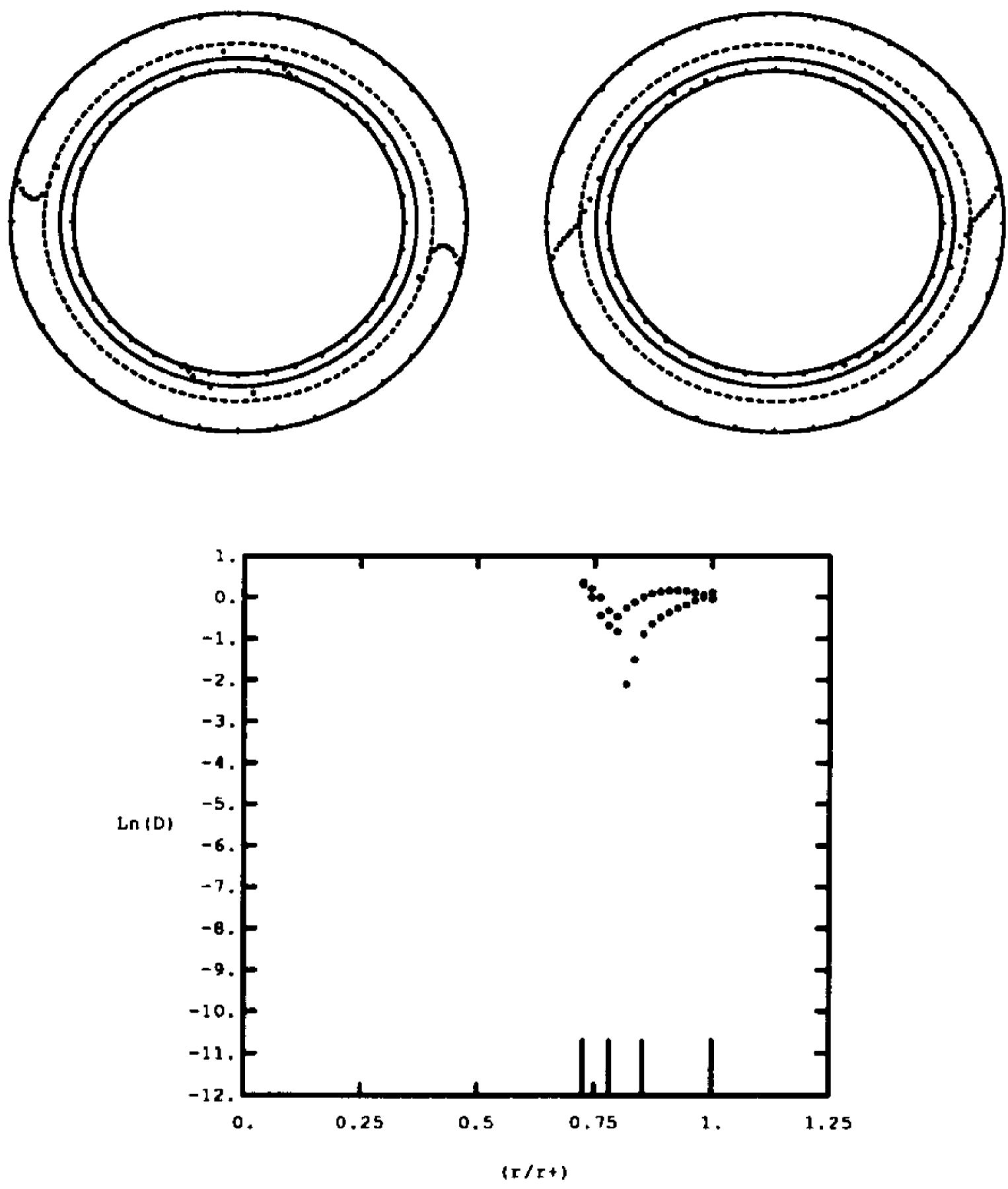

Figure 3.7b: The radial eigenfunction $\left(\phi_{2}-r\right)$ and amplitude as a function of radius $\left(D_{2}-r\right)$ of the $m=2$ Fourier mode illustrated as in Figure 2.3b for $M_{d} / M_{c}=5.0$, model \#6. 


\subsubsection{Systems with $M_{d} / M_{c}=1.0$}

At a mass ratio of 1.0 , we have identified model \#1 as the one that is marginally unstable to an $m=2$ mode distortion; that is, the critical value of $T /|W|=0.342$. This particular run was a very extended one, in part in order to see just how long the model would retain it's equilibrium structure. More importantly, due to the low growth rate of the model, an extended evolution was required to accurately determine the growth rate. After an initial 5 rotation periods of moderate amplitude growth, the model evolved over the remaining 18 rotation periods with a very low growth rate, $y_{2}(2)=0.004$ (almost indistinguishable from zero). The phase also eventually settled into a clearly defined pattern speed, with $y_{1}(2)=-0.85$. The growth of the $m=2$ mode over time is not characteristic of models we have seen up to this point. The instability appears to grow to some finite amplitude, the growth rate becoming essentially zero as this amplitude is reached. Given the low amplitude of the distortion, even after so long an evolution, it is not surprising to see the phase versus radius structure so raggedly defined as in figure $3.9 \mathrm{~b}$. It is apparent that the corotation point (and inner/outer Lindblad resonances) is having some effect. Also, there appears to be no rapid shift in phase similar to the $\pi / 2$ phase shift that was seen in the mass ratio 5.0 models. This behavior will be discussed further in $\$ 3.2 .3$, where models with a mass ratio of 0.2 are presented. (A number of models of mass ratio 0.2 show this behavior very distinctly.)

Model \#2 was also evolved farther than most models in this study. Figure 3.10a shows a somewhat long period oscillatory behavior in the $D_{2}-t$ plot, with a phase which becomes well defined early in the evolution, only to become disorganized during the low amplitude portion of the $D_{2}-t$ oscillation. Emerging from that decline in the amplitude, the pattern speed once again becomes well defined 
[the tabulated value of $y_{1}(2)$ is measured over this latter portion of the evolution]. The $\phi_{2}-r$ plots show a very gradual phase shift inside the pressure maximum - resembling to some extent the previously observed $\pi / 2$ shift - with the region outside of the pressure maximum showing a rather sweeping, spiral character. This is unlike the earlier models where the distortion at large radii exhibited little spiral character.

Models \#3 through \#5 all show the same basic characteristics as models \#3 \#5 of mass ratio 5.0. These models show a very clean $D_{2}-t$ behavior (with a hint of an oscillation in model \#5), and present well established pattern speeds. These models do show a $\pi / 2$ phase shift occurring inside the pressure maximum, but not one as sharply defined as in the higher mass ratio models. (Model \#5 exhibits a shift that is particularly broad, even at the midpoint of the evolution.) Model \#4 does not show the sharp minimum in the $D_{2}-r$ plot that is seen in other models. Model \#3 shows the minimum at high amplitude, but not at the intermediate stage. It is possible that such minima are oscillatory and the points in time selected for the $D_{2}-r$ plots are inadvertently at those points where the minimum is least visible.

Lastly, model \#6 shows an unmistakable oscillatory behavior in the $D_{2}-t$ plot, with the $m=4$ and $m=6$ modes exhibiting relatively large amplitudes. The $\phi_{2}-t$ plot shows a quite complicated structure, so the $y_{1}(2)$ parameter is ill defined (hence, the position of the corotation and inner/outer Lindblad resonances are also ill defined). There is a $\pi / 2$ phase shift occurring at or near the pressure maximum over relatively few radial grid zones ( 2 to 3 zones). 
Table 3.3

Initial Model Data for $M_{d} / M_{c}=1.0$

\begin{tabular}{cccc}
\hline \hline Model \# & $r_{-} / r_{+}$ & $r_{0} / r_{+}$ & $T /|W|$ \\
1 & 0.240 & 0.472 & 0.342 \\
2 & 0.277 & 0.527 & 0.356 \\
3 & 0.314 & 0.564 & 0.368 \\
4 & 0.333 & 0.583 & 0.373 \\
5 & 0.462 & 0.694 & 0.400 \\
6 & 0.611 & 0.787 & 0.417 \\
\hline
\end{tabular}


Table 3.4

$$
\mathrm{m}=2 \text { Eigenmode Character, } M_{d} / M_{c}=1.0
$$

\begin{tabular}{cccccc}
\hline \hline Model & $y_{1}$ & $y_{2}$ & $\Omega_{p} / \Omega_{0}$ & $r_{c r} / r_{0}$ & $r_{c r} / r_{+}$ \\
1 & -0.85 & 0.004 & 0.57 & 1.32 & 0.62 \\
2 & -0.77 & 0.060 & 0.61 & 1.27 & 0.67 \\
3 & -0.81 & 0.129 & 0.59 & 1.30 & 0.73 \\
4 & -0.82 & 0.195 & 0.58 & 1.30 & 0.76 \\
5 & -0.90 & 0.430 & 0.54 & 1.34 & 0.93 \\
6 & $(+1.02)$ & 0.597 & 1.51 & 0.81 & 0.64 \\
\hline
\end{tabular}



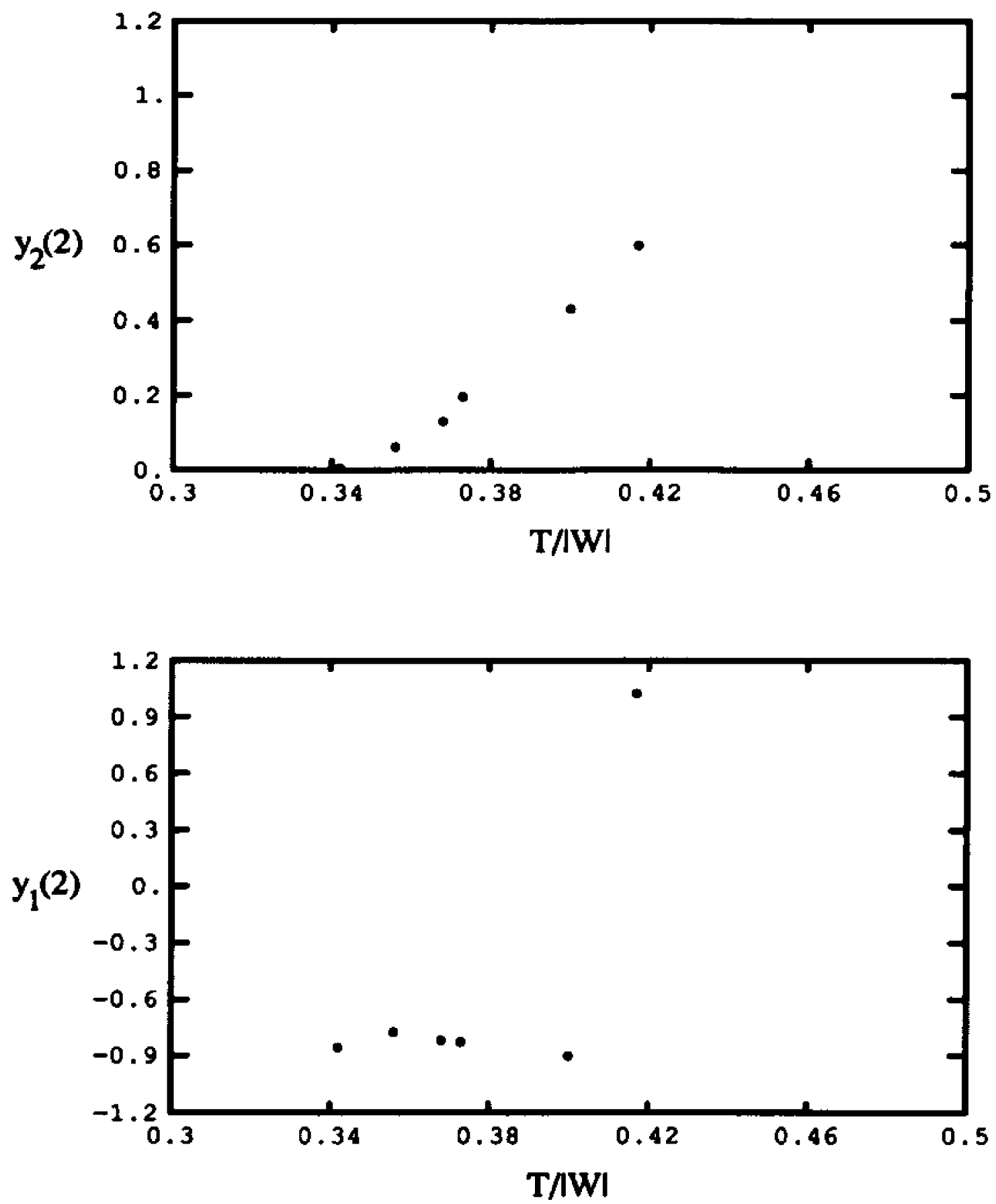

Figure 3.8: $y_{2}(2)$ and $y_{1}(2)$ as functions of $T /|W|$ for $M_{d} / M_{c}=1.0$ models. 

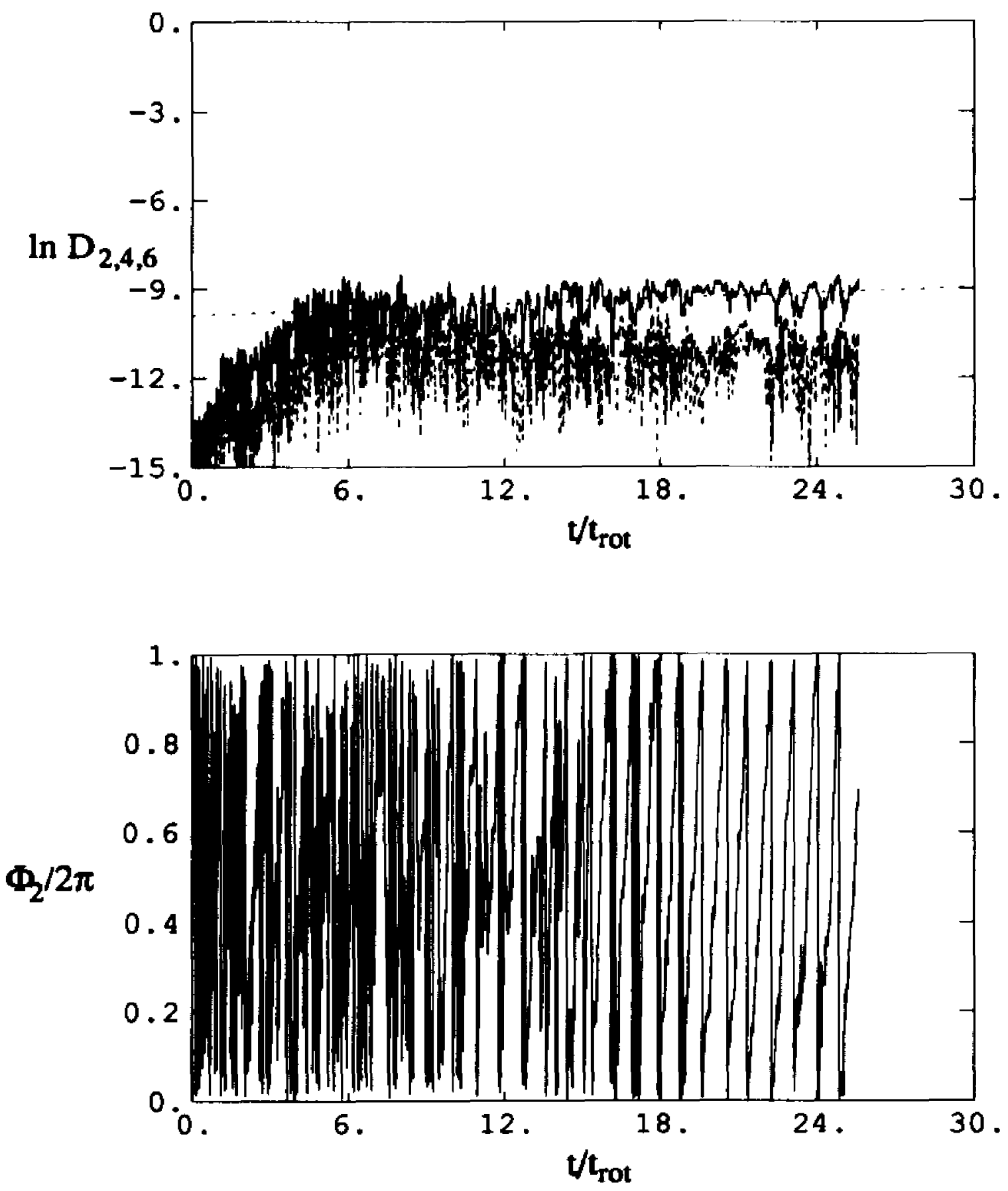

Figure 3.8a: The $m=2, m=4$, and $m=6$ Fourier amplitude behavior, and the $m=2$ phase behavior as a function of time, jllustrated as in Figure 2.3a for $M_{d} / M_{c}=1.0$, model $\# 1$. 

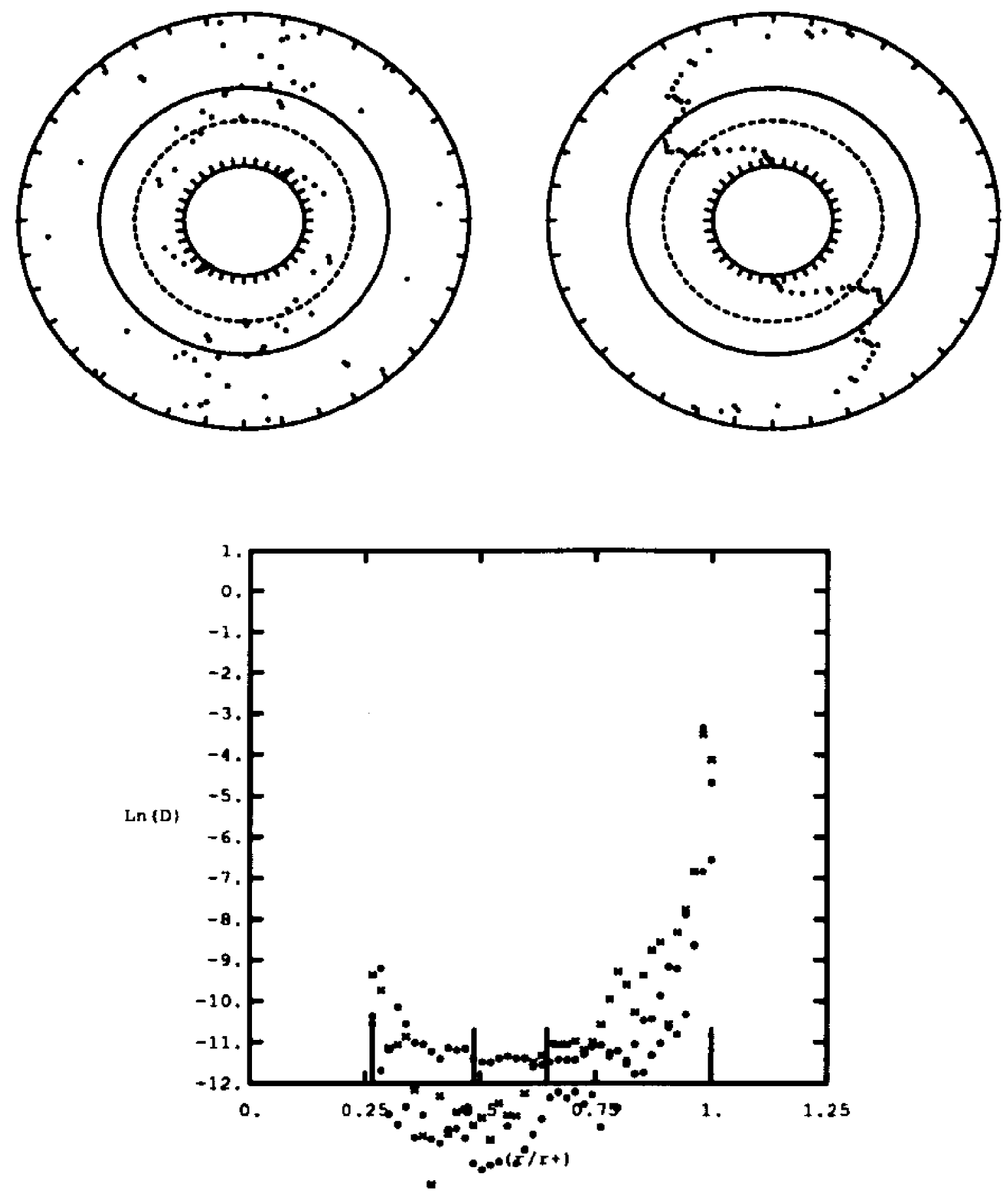

Figure 3.9b: The radial eigenfunction $\left(\phi_{2}-r\right)$ and amplitude as a function of radius $\left(D_{2}-r\right)$ of the $m=2$ Fourier mode illustrated as in Figure 2.3b for $M_{d} / M_{c}=1.0$, model \#1. 

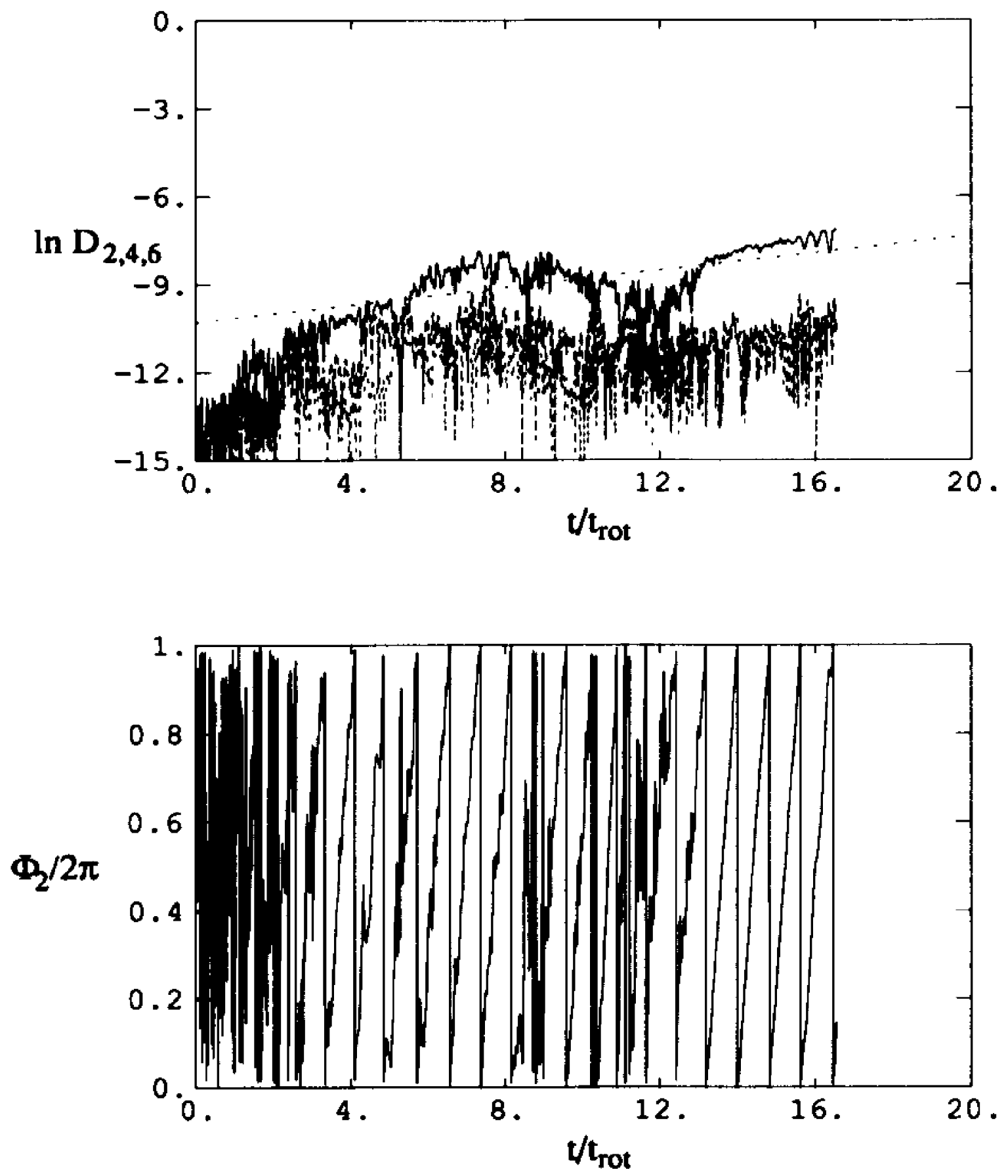

Figure 3.10a: The $m=2, m=4$, and $m=6$ Fourier amplitude behavior, and the $m=2$ phase behavior as a function of time, illustrated as in Figure 2.3a for $M_{d} / M_{c}=1.0$, model \#2. 

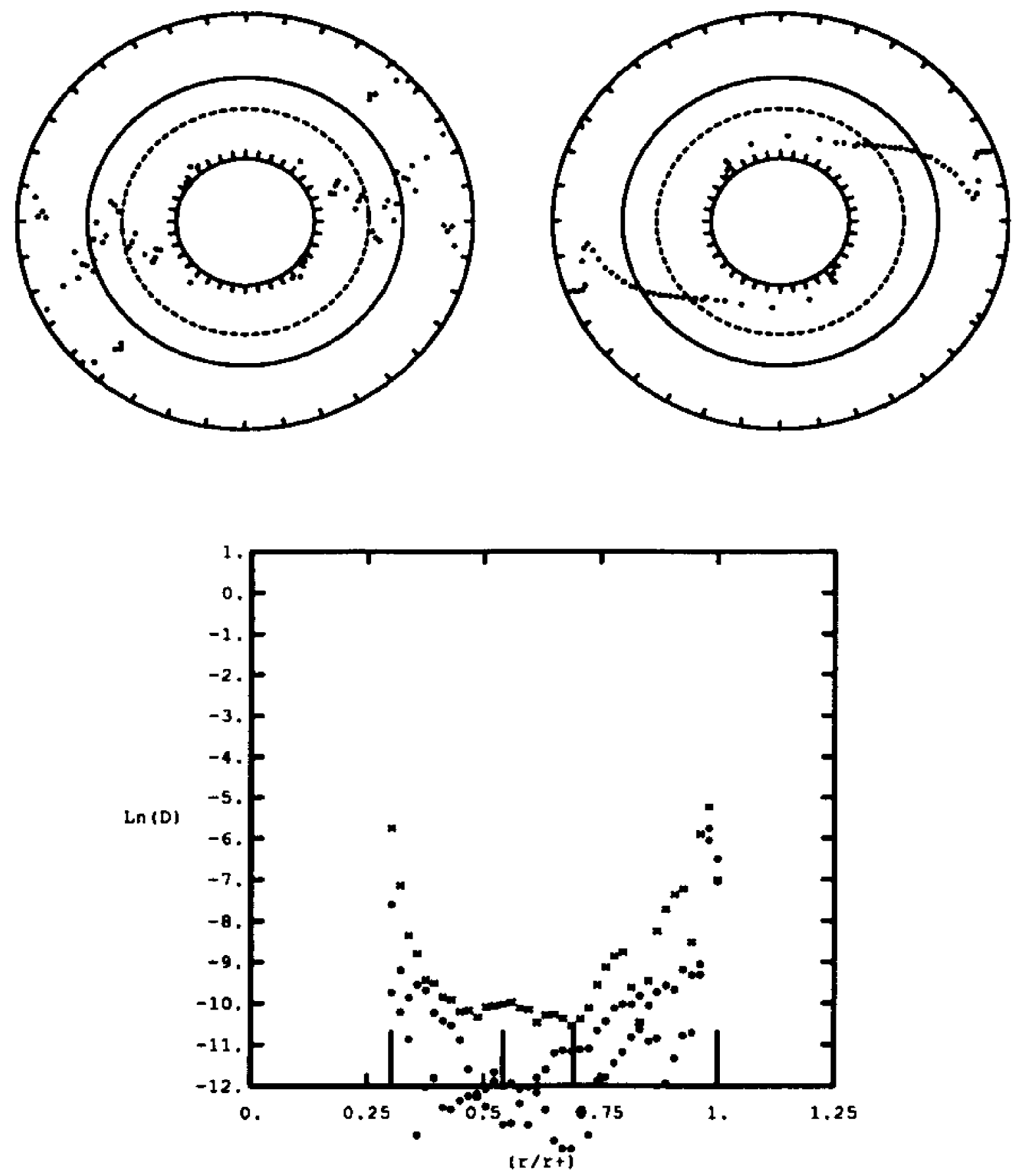

Figure 3.10b: The radial eigenfunction $\left(\phi_{2}-r\right)$ and amplitude as a function of radius $\left(D_{2}-r\right)$ of the $m=2$ Fourier mode illustrated as in Figure 2.3b for $M_{d} / M_{c}=1.0$, model \#2. 

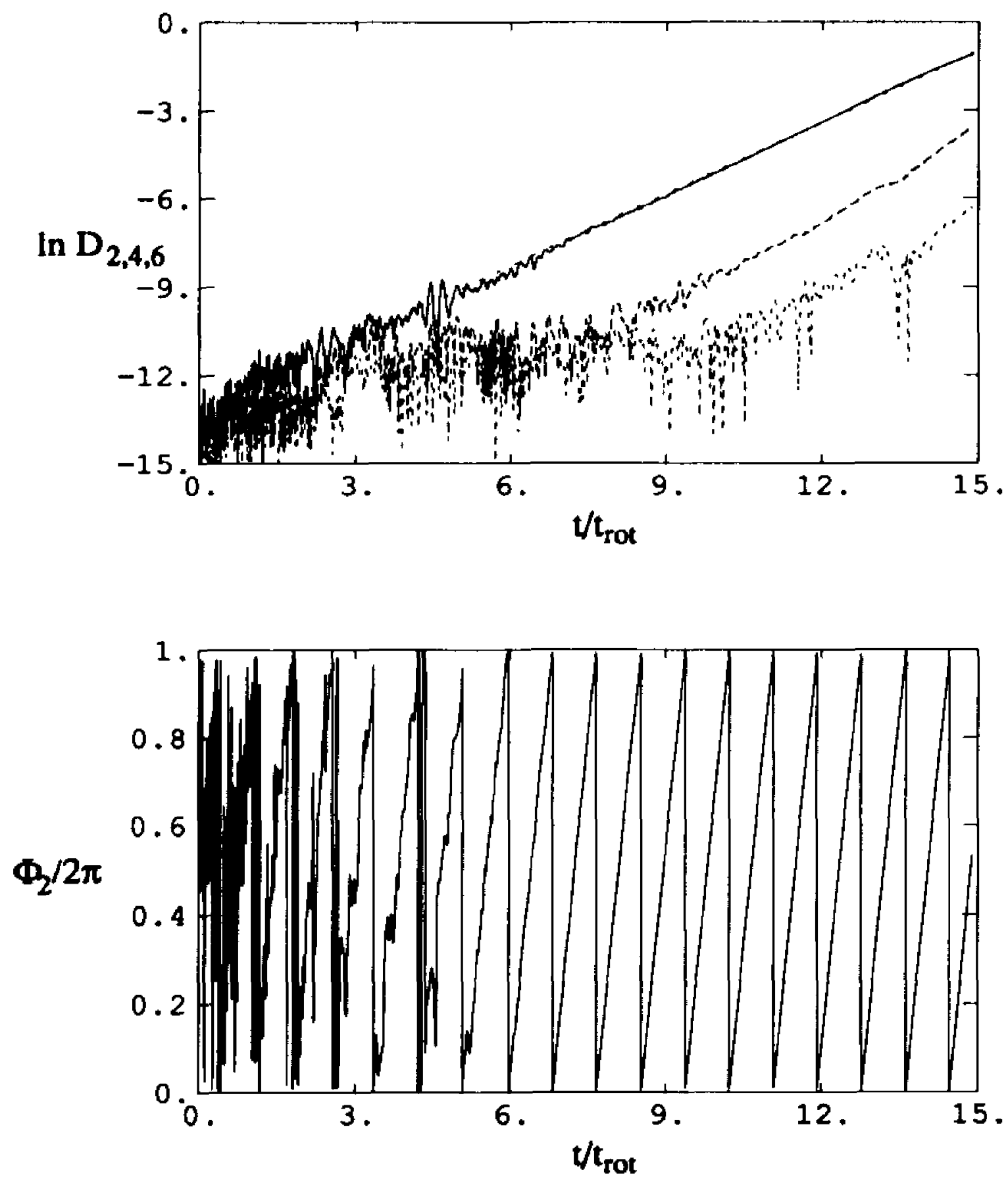

Figure 3.11a: The $m=2, m=4$, and $m=6$ Fourier amplitude behavior, and the $m=2$ phase behavior as a function of time, illustrated as in Figure 2.3a for $M_{d} / M_{\mathrm{c}}=1.0$, model \#3. 

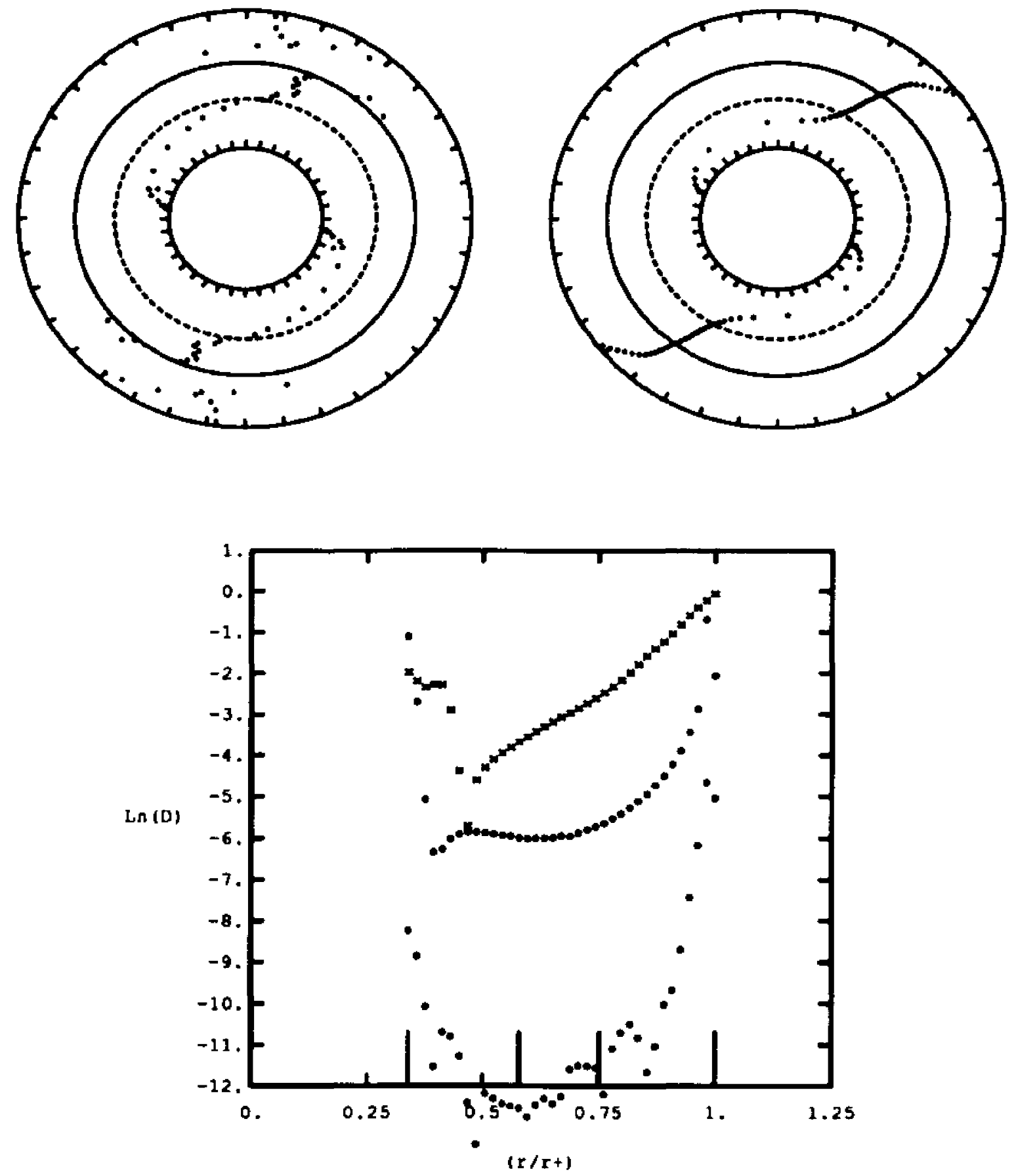

Figure 3.11b: The radial eigenfunction $\left(\phi_{2}-r\right)$ and amplitude as a function of radius $\left(D_{2}-r\right)$ of the $m=2$ Fourier mode illustrated as in Figure 2.3b for $M_{d} / M_{c}=1.0$, model \#3. 

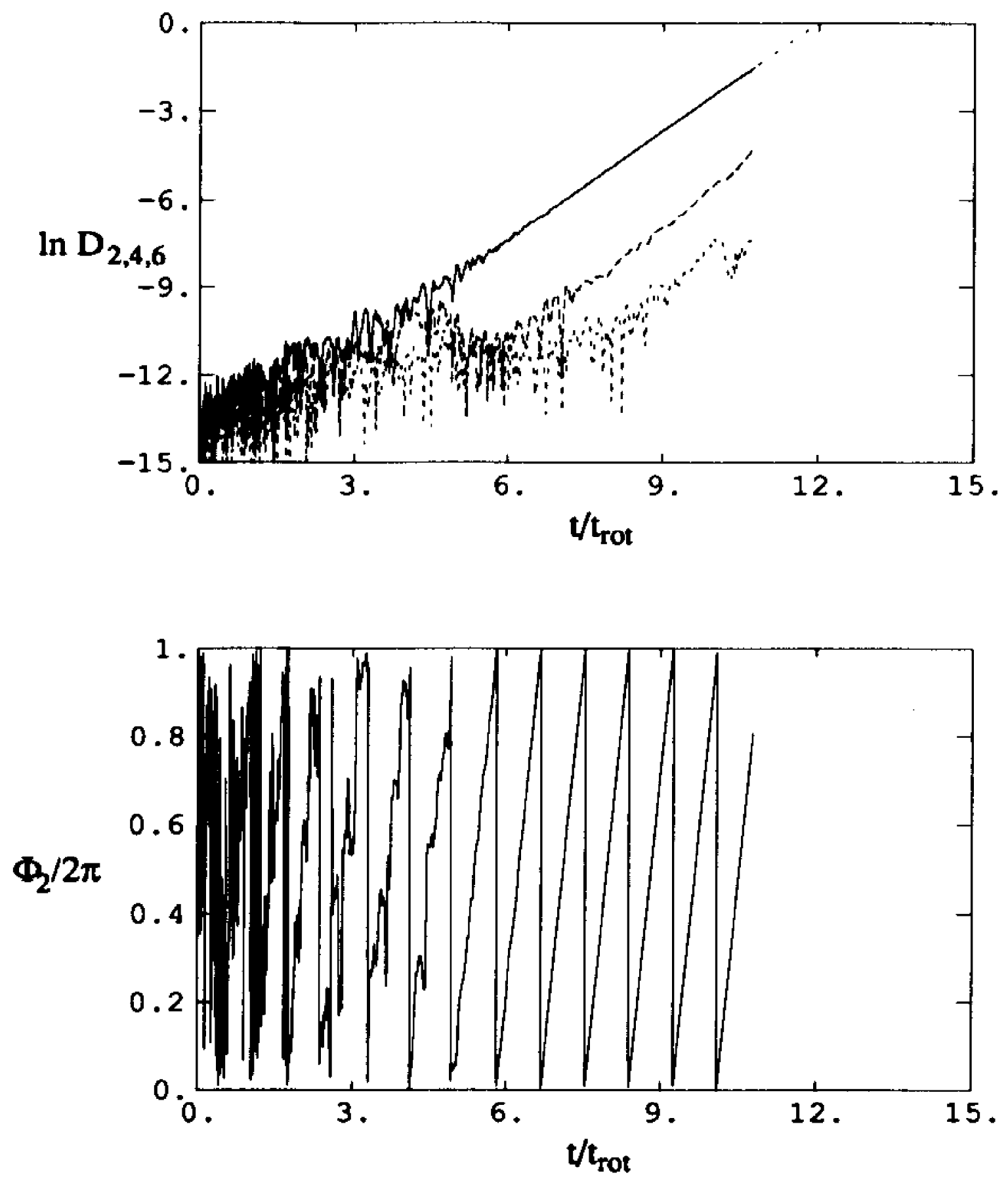

Figure 3.12a: The $m=2, m=4$, and $m=6$ Fourier amplitude behavior, and the $m=2$ phase behavior as a function of time, illustrated as in Figure 2.3a for $M_{d} / M_{c}=1.0$, model \#4. 

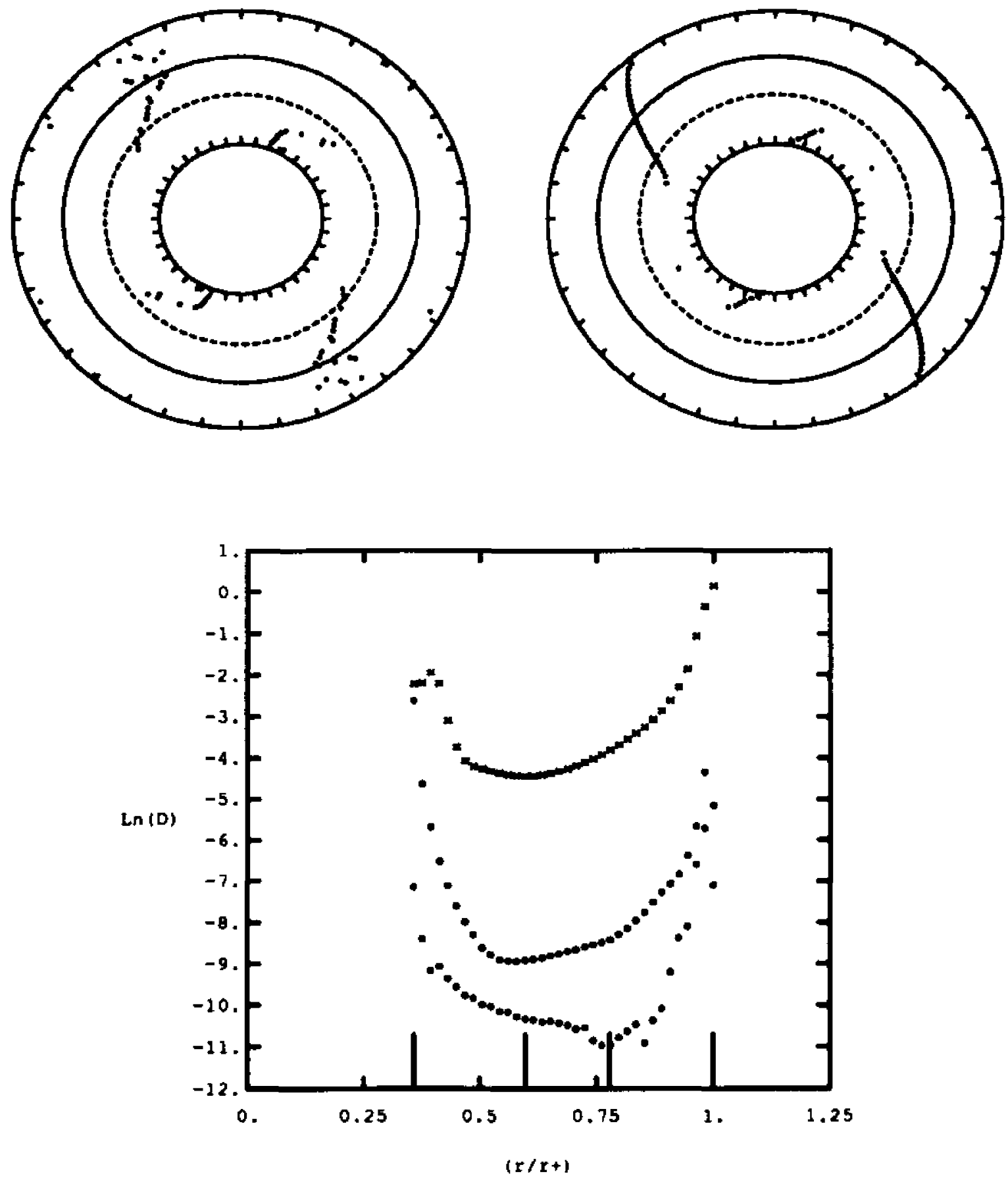

Figure 3.12b: The radial eigenfunction $\left(\phi_{2}-r\right)$ and amplitude as a function of radius $\left(D_{2}-r\right)$ of the $m=2$ Fourier mode illustrated as in Figure 2.3b for $M_{d} / M_{c}=1.0$, model \#4. 

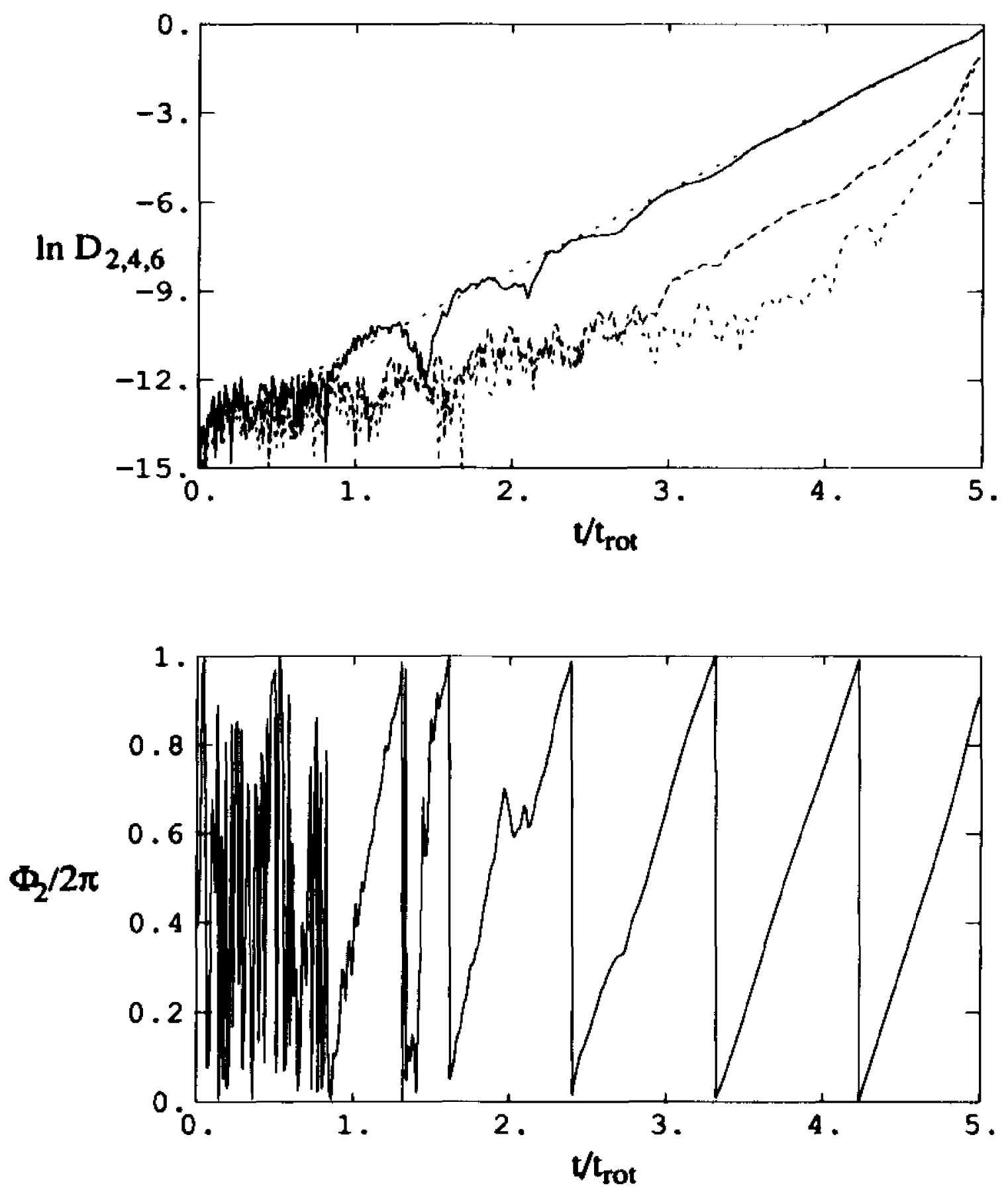

Figure 3.13a: The $m=2, m=4$, and $m=6$ Fourier amplitude behavior, and the $m=2$ phase behavior as a function of time, illustrated as in Figure 2.3a for $M_{d} / M_{c}=1.0$, model \#5. 

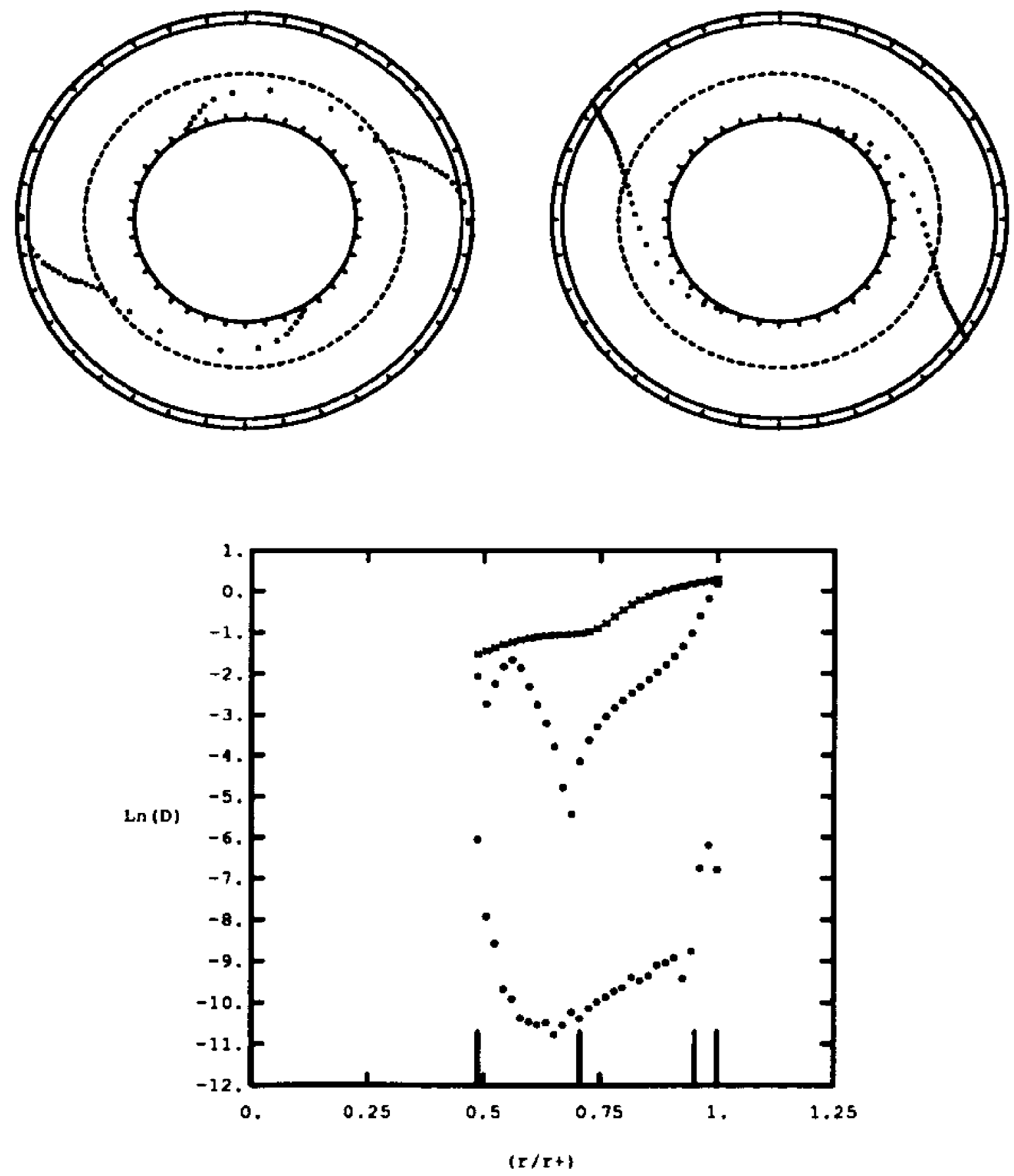

Figure 3.13b: The radial eigenfunction $\left(\phi_{2}-r\right)$ and amplitude as a function of radius $\left(D_{2}-r\right)$ of the $m=2$ Fourier mode illustrated as in Figure 2.3b for $M_{d} / M_{c}=1.0$, model \#5. 

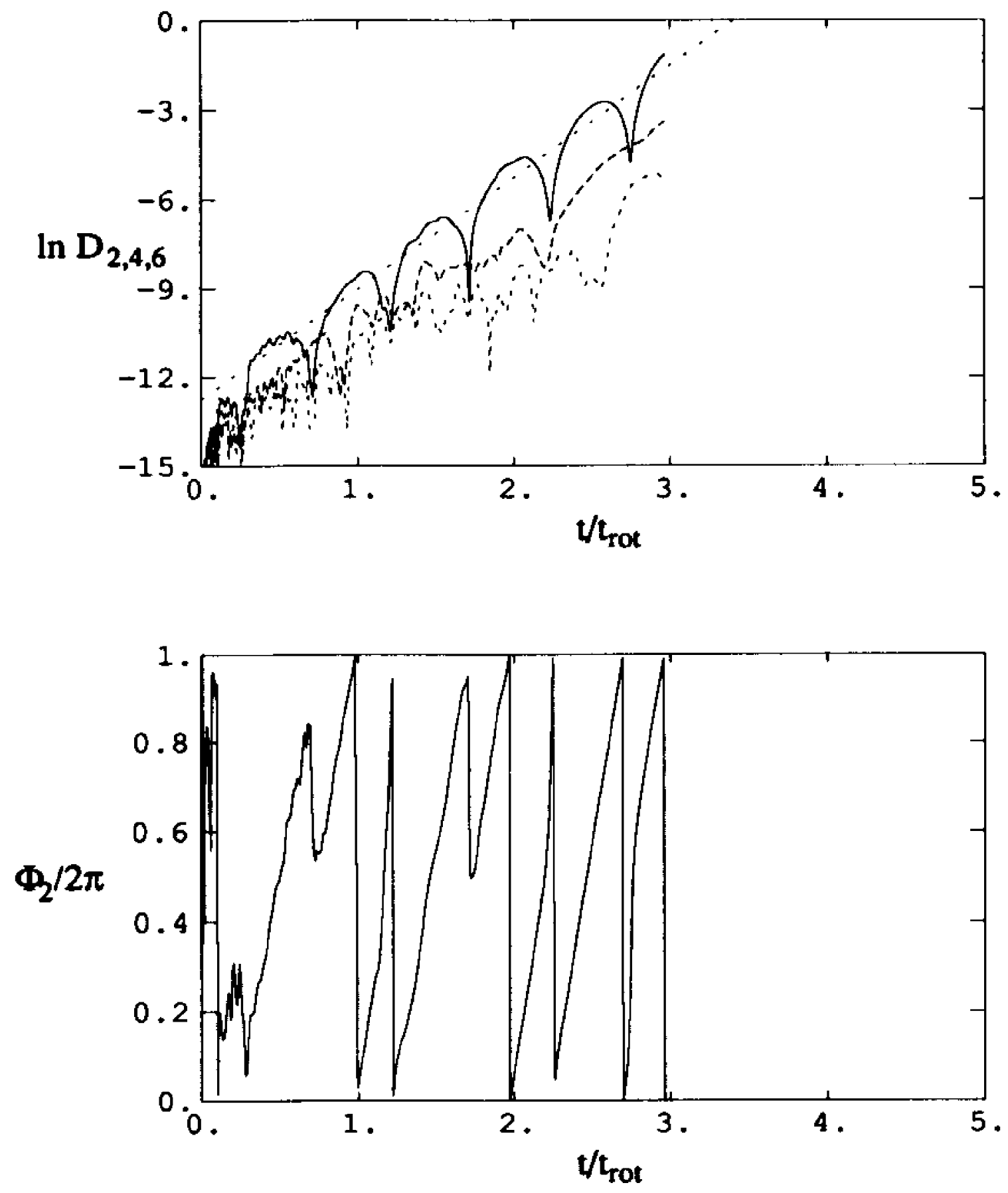

Figure 3.14a: The $m=2, m=4$, and $m=6$ Fourier amplitude behavior, and the $m=2$ phase behavior as a function of time, illustrated as in Figure $2.3 \mathrm{a}$ for $M_{\mathrm{d}} / M_{\mathrm{c}}=1.0$, model \#6. 

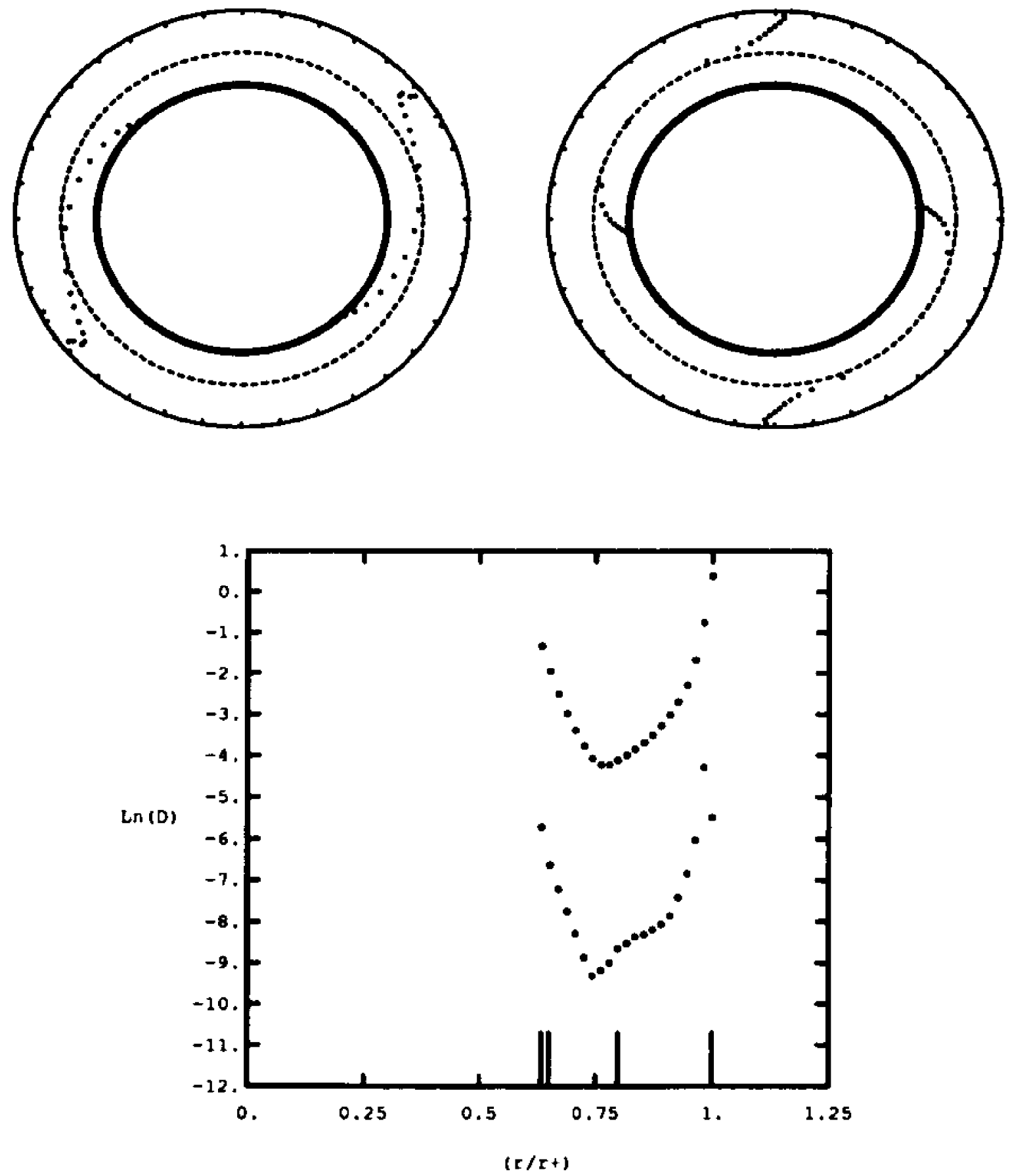

Figure 3.14b: The radial eigenfunction $\left(\phi_{2}-r\right)$ and amplitude as a function of radius $\left(D_{2}-r\right)$ of the $m=2$ Fourier mode illustrated as in Figure 2.3b for $M_{d} / M_{c}=1.0$, model \#6. 


\subsubsection{Systems with $M_{d} / M_{c}=0.2$}

Examining Figure 3.15, it is clear that the series of models with $M_{d} / M_{c}=0.2$ exhibits a different kind of behavior from the systems with larger mass ratios that were examined earlier. When plotted versus $T /|W|$, for example, the $y_{2}(2)$ parameter does not define a simple curve that drops monotonically to zero at a critically unstable value of $T /|W|$. Instead, $y_{2}$ shows a more complicated functional dependence on $T /|W|$, as evidenced by the dip in the growth rates between model $\# 3$ and model \#6. This could be indicative of a particular instability dying out as $T /|W|$ increases, and being replaced by another mode. The pattern speed parameter, $y_{1}(2)$, also varies widely from one model to the next.

It appears as though the critical value of $T /|W|$ lies somewhere between 0.42 and 0.43 ; that is, the marginally unstable model lies somewhere between models \#1 and \#2. After an initial period of growth early in the evolution of model \#1, the amplitude of the $m=2$ mode actually decreases slowly, and appears to approach a constant amplitude. This results in a very small negative growth rate, $y_{2}(2)=$ -0.004 . The constant amplitude, coupled with a very small negative growth rate is indicative of a dynamically stable model. The $y_{1}(2)$ parameter reported in Table 3.6 for model \#1 was determined from the coherent portion of the $\phi_{2}-t$ plot early in the evolution and, hence, should be taken as uncertain. The $\phi_{2}-r$ plots in Figure 3.16b show a very broad, two-armed spiral late in the evolution, not an abrupt shift in phase as reported in other systems. The evolution of this spiral has not been examined in any detail.

Model \#2 shows a very ragged, oscillatory growth, with the phase well defined but varying in frequency at different points in the evolution. Thus, the $v_{1}(2)$ parameter depends critically on where one measures it; the value given in Table 3.6 
should only be considered approximate. The $\phi_{2}-r$ plot for model \#2 again shows the $\pi / 2$ phase shift at the pressure maximum. The low amplitude of the distortion precludes any meaningful discussion of the $D_{2}-r$ plot in Figure 3.17b.

Models \#3 and \#4 both show an initial growth followed by a leveling off of the amplitude, much like model \#1 of mass ratio 1.0 (and, perhaps, model \#1 of mass ratio 0.2). As indicated by the dotted line in Figs. 3.18a and 3.19a, the $y_{2}(2)$ parameter is determined from the early period of growth. (Over the later period of the evolution, $y_{2}(2)$ tends toward zero, as in model \#1.) The $\phi_{2}-t$ behavior is very well defined in these models, so the $y_{1}(2)$ parameter is easily determined. The values obtained, however, differ markedly from one another: model \#3 has $y_{1}(2)=+0.10$, and model \#4 has $y_{1}(2)=+0.29$. The $\phi_{2}-r$ behavior of these two models is very similar. When compared to higher mass ratio systems, the most distinguishing feature is the lack of an abrupt phase shift of any kind. The phase of the $m=2$ distortion is "bar-like" across the entire model, with the exception of the outermost regions of model \#4. Notice, also, that in these two models, $r_{c r}$ appears to coincide very closely with $r_{0}$.

Why these models, as well as model \#1 of mass ratio 1.0, exhibit this type of behavior is subject to debate. Several ideas have been put forward and need to be examined. Perhaps the most intriguing idea is that the disks may be accreting material onto the central object, and this accretion may be enough to stabilize the model against further growth of the $m=2$ instability. (Accretion behavior occurs in almost all models when the distortions grow to nonlinear amplitudes; this has been observed particularly well in model \#3 of the mass ratio 1.0 disks while experimenting with imaging and animation techniques.) These models should be examined in greater detail as the steady accretion scenario also would be of considerable interest in other astrophysical contexts; for example, white dwarf or neutron star systems. 
The more probable explanation for the behavior of these models is that it is due to a nonlinear mode-coupling effect (see chapter 7 of Drazin and Reid, 1981, for a more complete discussion of nonlinear analyses). In linear stability theory, a spectrum of independent modes develops in the fluid system, each with a distinct growth rate and pattern speed dependent upon some parameter of the system. (In traditional fluid systems, this parameter is usually the Reynolds number; in our particular case, this parameter is taken to be the value of $T /|W|$.) We know that when $T /|W|$ is less than some critical value, the system is stable; as $T /|W|$ is increased above the critical value, each independent mode begins to grow in sequence. We have observed this in the models we have evolved. For example, model \#3 of mass ratio 5.0 has a clearly defined $m=2$ mode dominating the evolution, with a low growth rate $m=4$ mode, followed by a negligible $m=6$ mode. Examining model \#5, with a higher value of $T /|W|$, the $m=4$ mode becomes better defined, with a slightly higher growth rate, while $m=6$ is still negligible. In model \#6, the $m=6$ has developed an appreciable growth rate, while in model \#7, the $m=2, m=4$, and $m=6$ modes all have substantial growth rates. This behavior has been referred to in prior sections with references to the behavior observed by Kojima (1986).

A nonlinear analysis indicates that in cases where $T /|W|$ is near the critical value, there is a nonlinear self-interaction of the dominant mode. This results in the generation of harmonics within the system, moderating the exponential growth of the mode, described through the Landau equation

$$
\frac{d|A|^{2}}{d t}=2 \sigma|A|^{2}-l|A|^{4}
$$

(Drazin and Reid 1981, equation 49.3), where $|A|$ is the amplitude of the dominant mode, $\sigma$ is essentially the same as $\operatorname{Im}(\omega)$ defined in Chapter 2 , and $l$ is the Landau 
constant. From this equation emerges a number of possibilities which depend upon the signs of $\sigma$ and $l$. The case which is of interest to us is the case where the growth rate is positive $(\sigma>0)$ and $l>0$; this case is called supercritical stability. The system is linearly unstable, but the amplitude of the disturbance (mode) grows asymptotically toward a constant value, $A_{c}$ given by

$$
|A| \rightarrow A_{e} \equiv\left(\frac{2 a}{l}\right)^{1 / 2}
$$

(Drazin and Reid 1981, equation 49.5). Phenomenologically, at least, this is what is observed for the models in question: the $m=2$ mode grows from a very low initial amplitude, and equilibrates at some constant amplitude. The derivation of the Landau equation, however, is a process much more complicated than the linear analyses normally used in this field. Hence, while it may be possible to determine the growth rate from the initial growth of the model, the Landau constant, $l$, is difficult to determine from the initial set of differential equations governing self-gravitating disk structures. We simply suggest that supercritical stability is consistent with the observed $D_{2}-t$ behavior in models \#3 and \#4.

The first half of the evolution of model \#5 is very incoherent. From the $\phi_{2}-t$ plot illustrated in Figure 3.20a, there is a brief period of apparent coherent growth from time $t / t_{\text {rot }}=1$ to $t / t_{\text {rot }}=3$. Over such a brief time period, not a great deal can be said, but the $y_{1}(2)$ parameter that results from that time period, $y_{1}(2)=0.297$, is very close to the the $y_{1}(2)$ value derived for model $\# 4, y_{1}(2)=0.291$. Hence, this model may have started its evolution in a similar manner to the previous model, growing for a short period of time followed by a stabilization of the instability at a finite amplitude. For five rotation periods following that early time, however, the amplitude is not smoothly changing over time and the phase is ill-defined. At $t / t_{\text {rot }}=8$, a clear exponential growth of the $m=2$ mode emerges with a well defined 
pattern speed, not unlike that of the mass ratio $\infty$ and mass ratio 5.0 models. The $\phi_{2}-r$ plots of Figure 3.20b show the characteristic $\pi / 2$ phase shift, while the $D_{2}-r$ plot shows a minimum near the pressure maximum. Therefore, the latter portion of the evolution of this model appears to be very similar to the mass ratio $\infty$ and 5.0 evolutions.

Models \#6 and \#7 show the oscillatory amplitude behavior that we saw in model \#6 of mass ratio 5.0. This oscillatory behavior is probably due to a nonlinear interaction of a different type than that discussed above. In the $D_{2}-t$ plots of models \#6 and \#7 (Figures $3.21 \mathrm{a}$ and $3.22 \mathrm{a}$ ), the $m=2$ and $m=4$ modes are oscillating with similar periods; hence, there appears to be some coupling of these modes. The $\phi_{2}-t$ behavior is somewhat ill-defined. (A value of $y_{1}(2)$ has been tabulated for model \#6, but this should only be regarded as an estimate.) The $\phi_{2}-r$ function for these two models shows the characteristic $\pi / 2$ phase shift. Finally, note that the higher order modes are developing appreciable amplitudes, with $m=4$ dominating in model \#7. 
Table 3.5

Initial Model Data for $M_{d} / M_{c}=0.2$

\begin{tabular}{cccl}
\hline \hline Model \# & $r_{-} / r_{+}$ & $r_{0} / r_{+}$ & $T /|W|$ \\
1 & 0.314 & 0.527 & 0.422 \\
2 & 0.351 & 0.583 & 0.432 \\
3 & 0.425 & 0.657 & 0.447 \\
4 & 0.462 & 0.694 & 0.453 \\
5 & 0.518 & 0.731 & 0.460 \\
6 & 0.611 & 0.805 & 0.468 \\
7 & 0.740 & 0.879 & 0.475 \\
\hline
\end{tabular}


Table 3.6

$\mathrm{m}=2$ Eigenmode Character, $M_{d} / M_{c}=0.2$

\begin{tabular}{cccccc}
\hline \hline Model & $y_{1}$ & $y_{2}$ & $\Omega_{p} / \Omega_{0}$ & $r_{c r} / r_{0}$ & $r_{c r} / r_{+}$ \\
1 & $(+0.03)$ & -0.004 & 1.01 & 0.99 & 0.52 \\
2 & $(-0.23)$ & 0.041 & 0.88 & 1.06 & 0.62 \\
3 & +0.10 & {$[0.277]$} & 1.05 & 0.97 & 0.64 \\
4 & +0.29 & {$[0.209]$} & 1.14 & 0.93 & 0.64 \\
5 & -0.74 & 0.134 & 0.62 & 1.26 & 0.92 \\
6 & $(+0.67)$ & 0.295 & 1.33 & 0.86 & 0.69 \\
7 & - & 0.402 & - & - & - \\
\hline
\end{tabular}



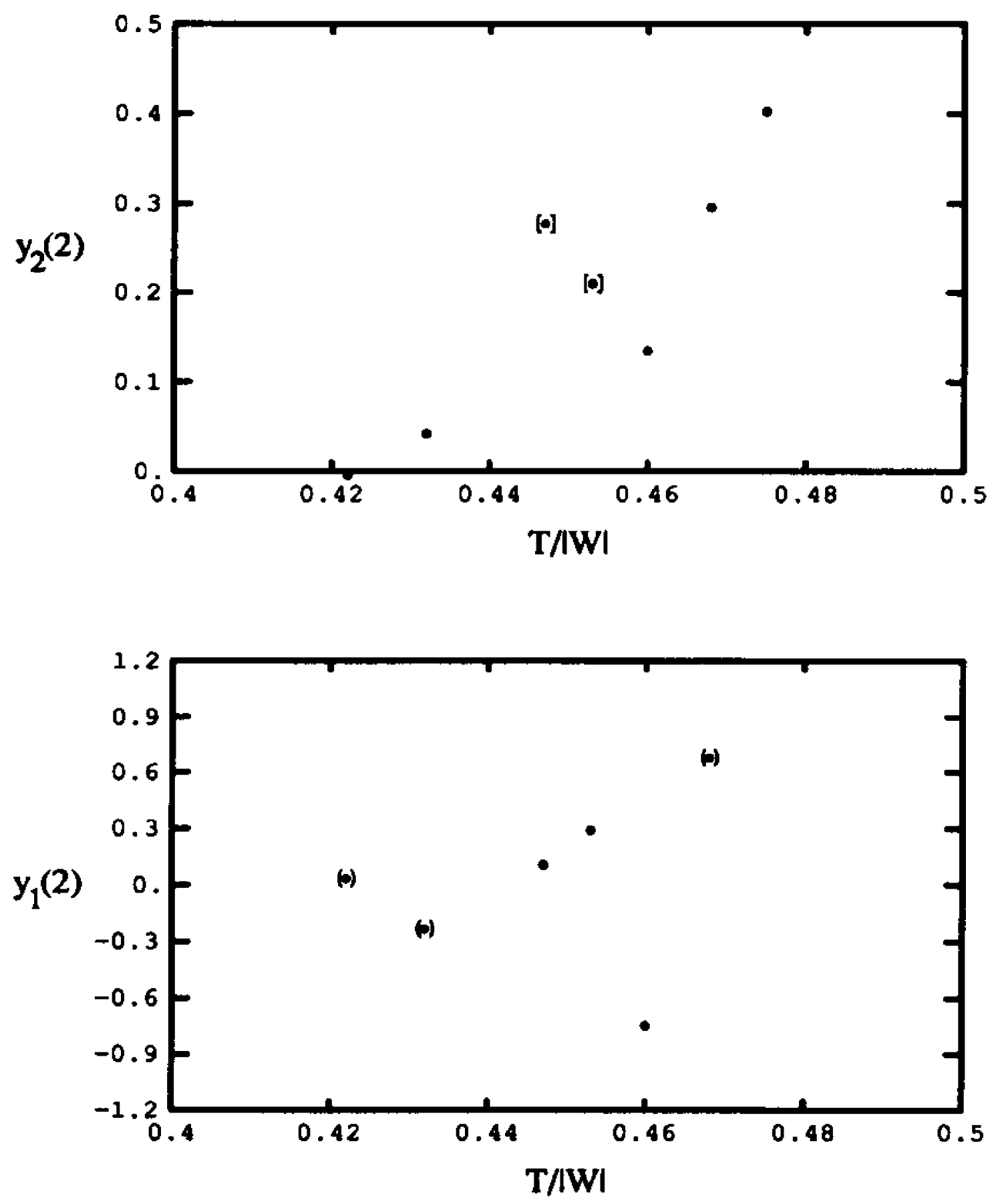

Figure 3.15: $y_{2}(2)$ and $y_{1}(2)$ as functions of $T /|W|$ for $M_{d} / M_{c}=0.2$ models. 

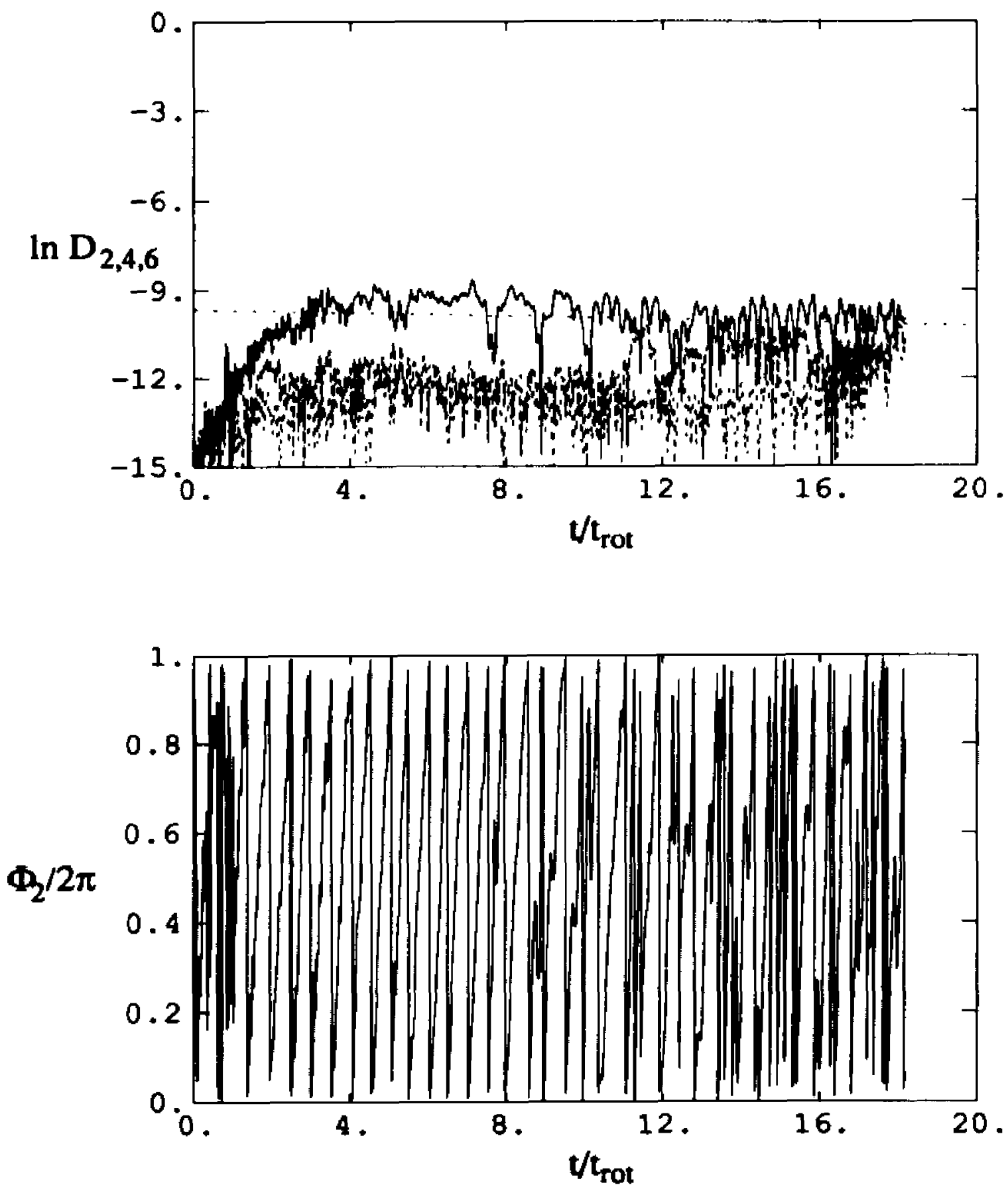

Figure 3.16a: The $m=2, m=4$, and $m=6$ Fourier amplitude behavior, and the $m=2$ phase behavior as a function of time, illustrated as in Figure 2.3a for $M_{d} / M_{c}=0.2$, model \#1. 

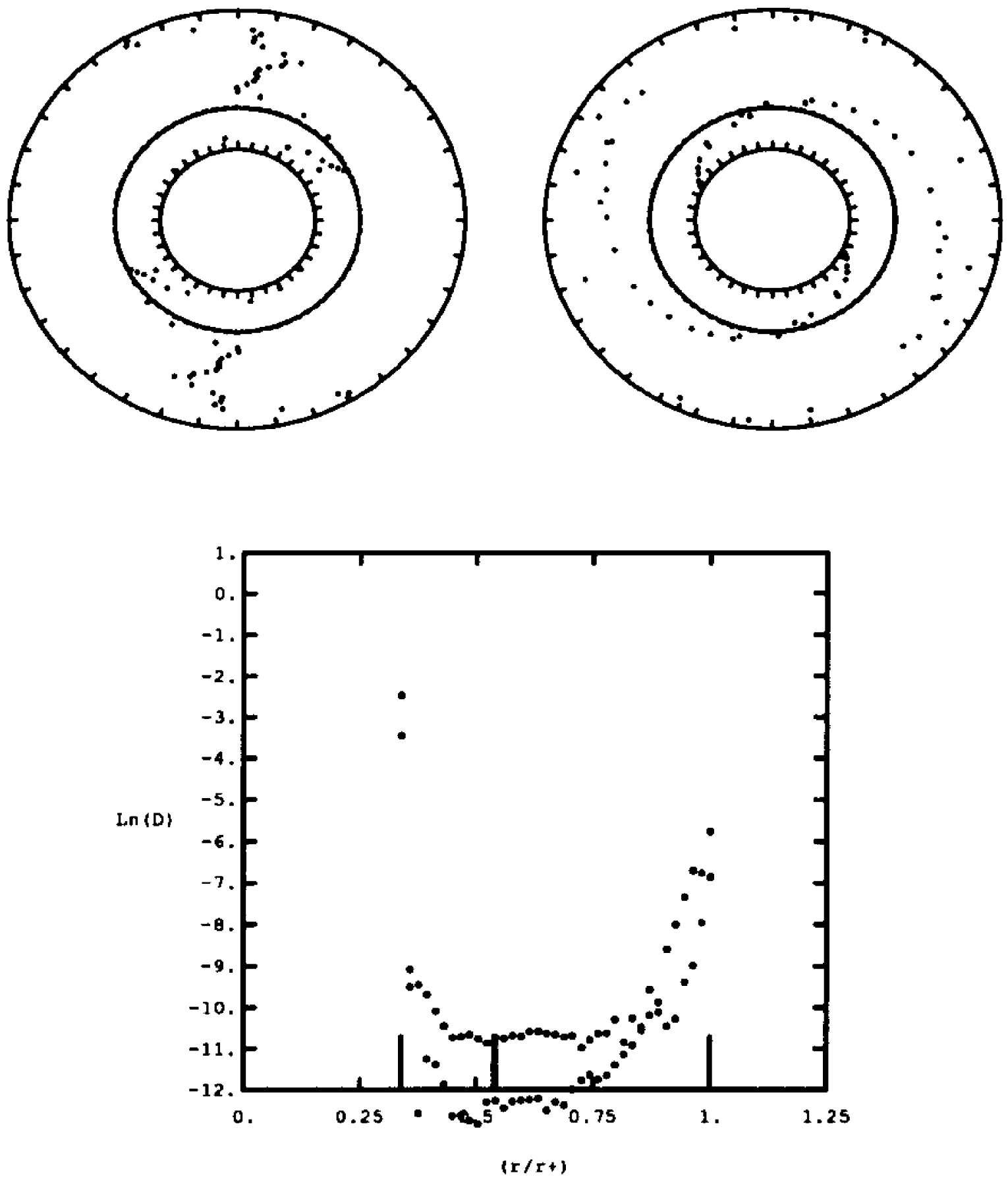

Figure 3.16b: The radial eigenfunction $\left(\phi_{2}-r\right)$ and amplitude as a function of radius $\left(D_{2}-r\right)$ of the $m=2$ Fourier mode illustrated as in Figure 2.3b for $M_{d} / M_{c}=0.2$, model \#1. 

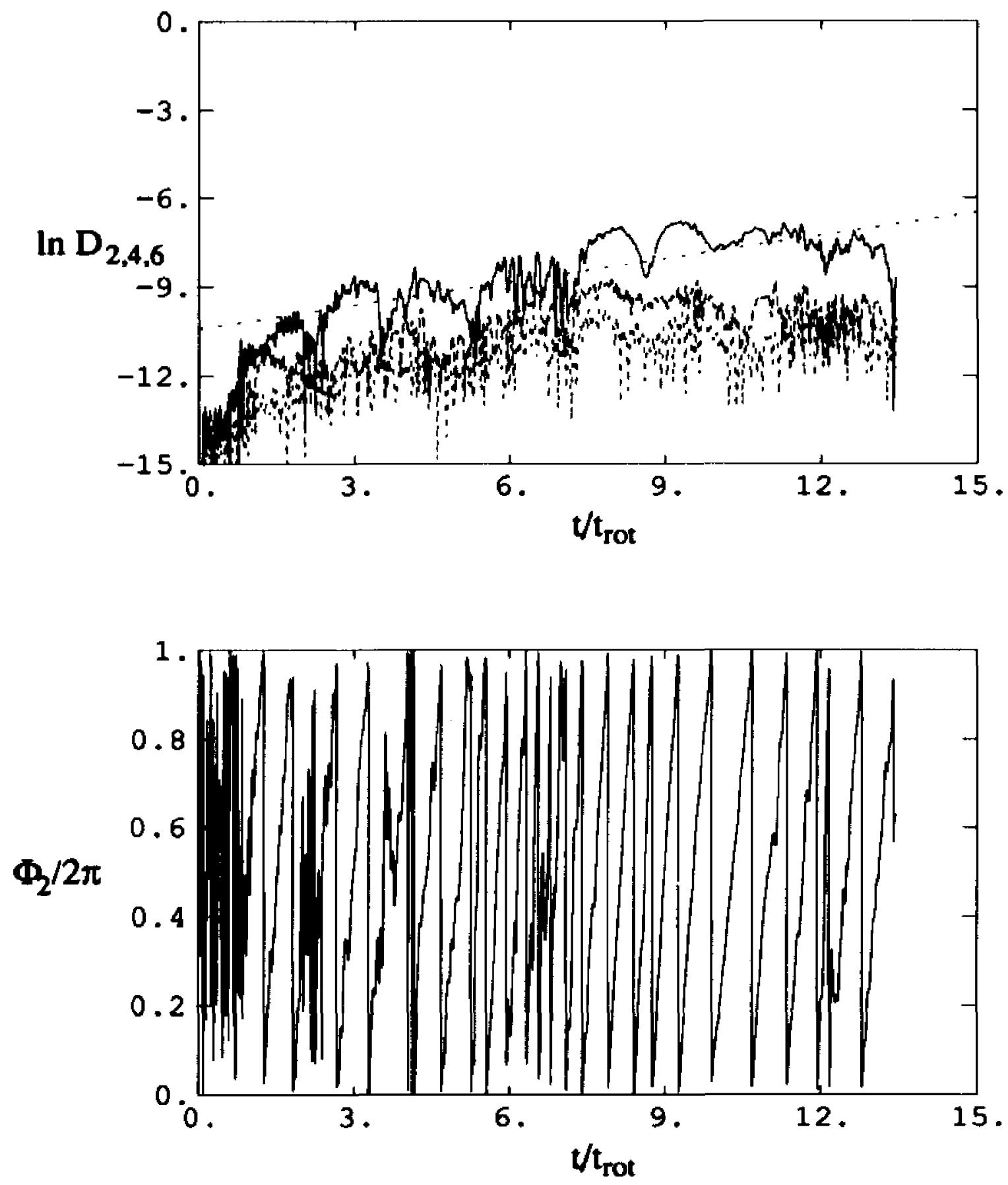

Figure 3.17a: The $m=2, m=4$, and $m=6$ Fourier amplitude behavior, and the $m=2$ phase behavior as a function of time, illustrated as in Figure 2.3a for $M_{d} / M_{c}=0.2$, model \#2. 

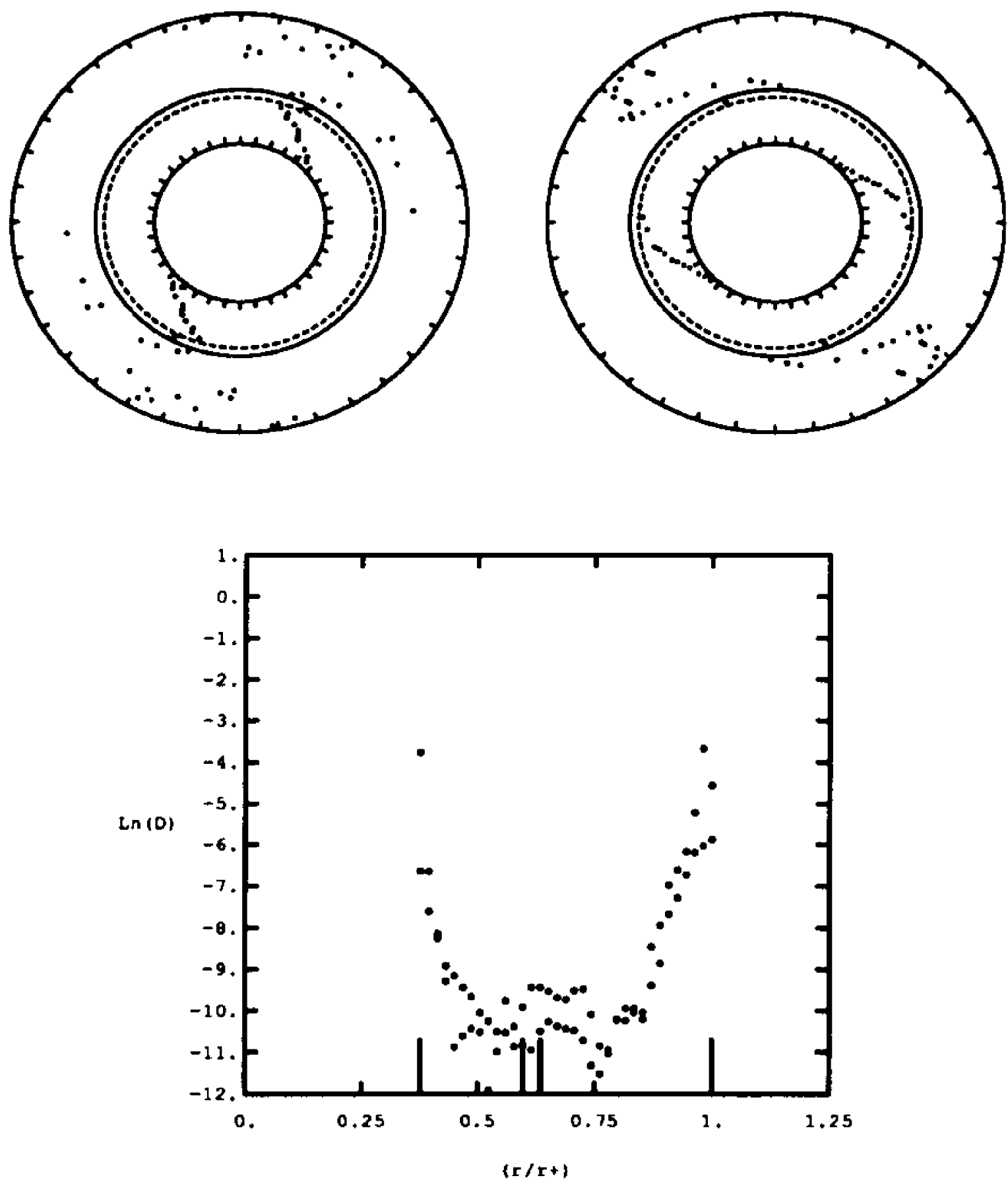

Figure 3.17b: The radial eigenfunction $\left(\phi_{2}-r\right)$ and amplitude as a function of radjus $\left(D_{2}-r\right)$ of the $m=2$ Fourier mode illustrated as in Figure 2.3b for $M_{d} / M_{c}=0.2$, model \#2. 

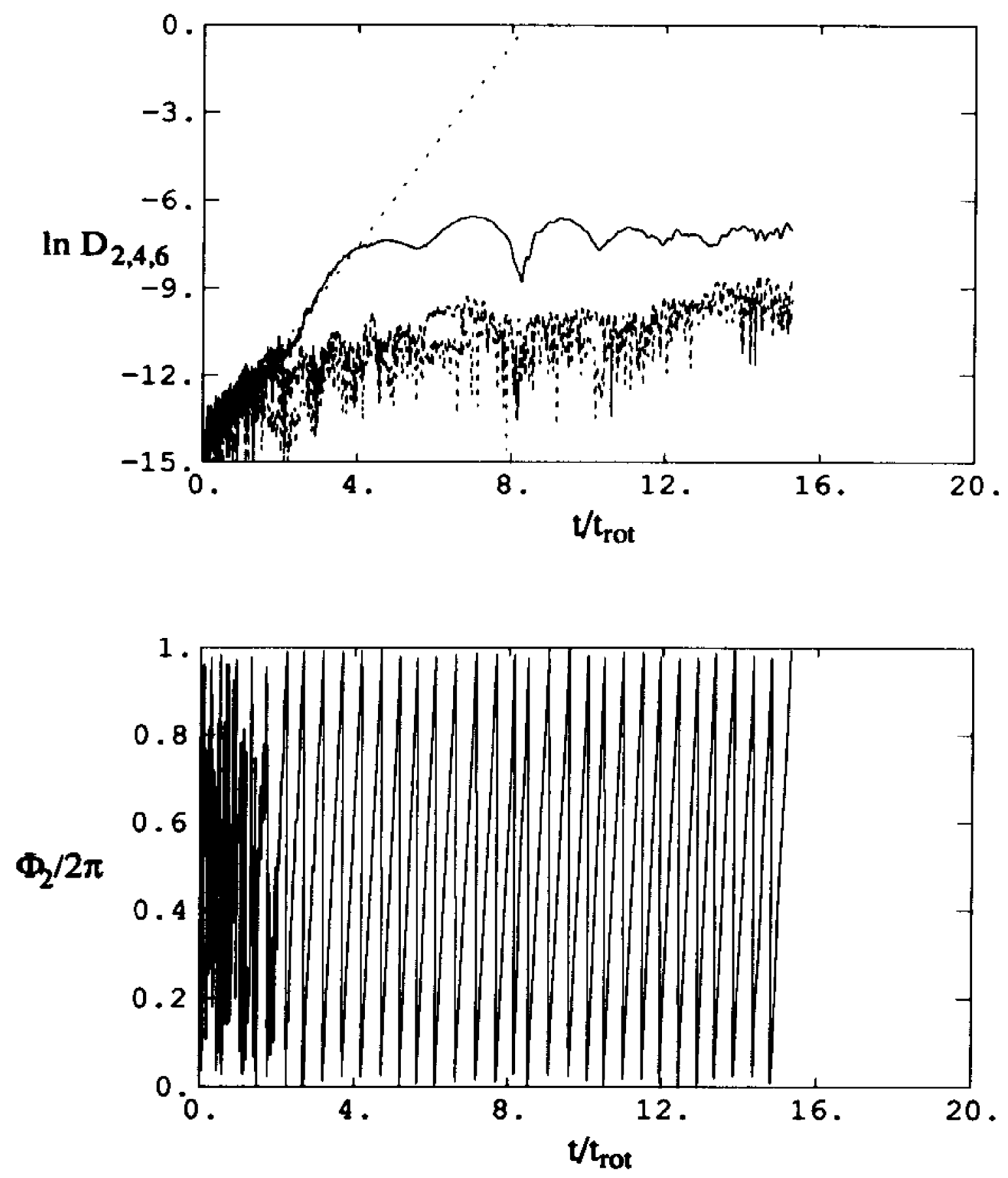

Figure 3.18a: The $m=2, m=4$, and $m=6$ Fourier amplitude behavior, and the $m=2$ phase behavior as a function of time, illustrated as in Figure 2.3a for $M_{d} / M_{c}=0.2$, model \#3. This model is identified in the text as an ideal example of supercritical stability. 

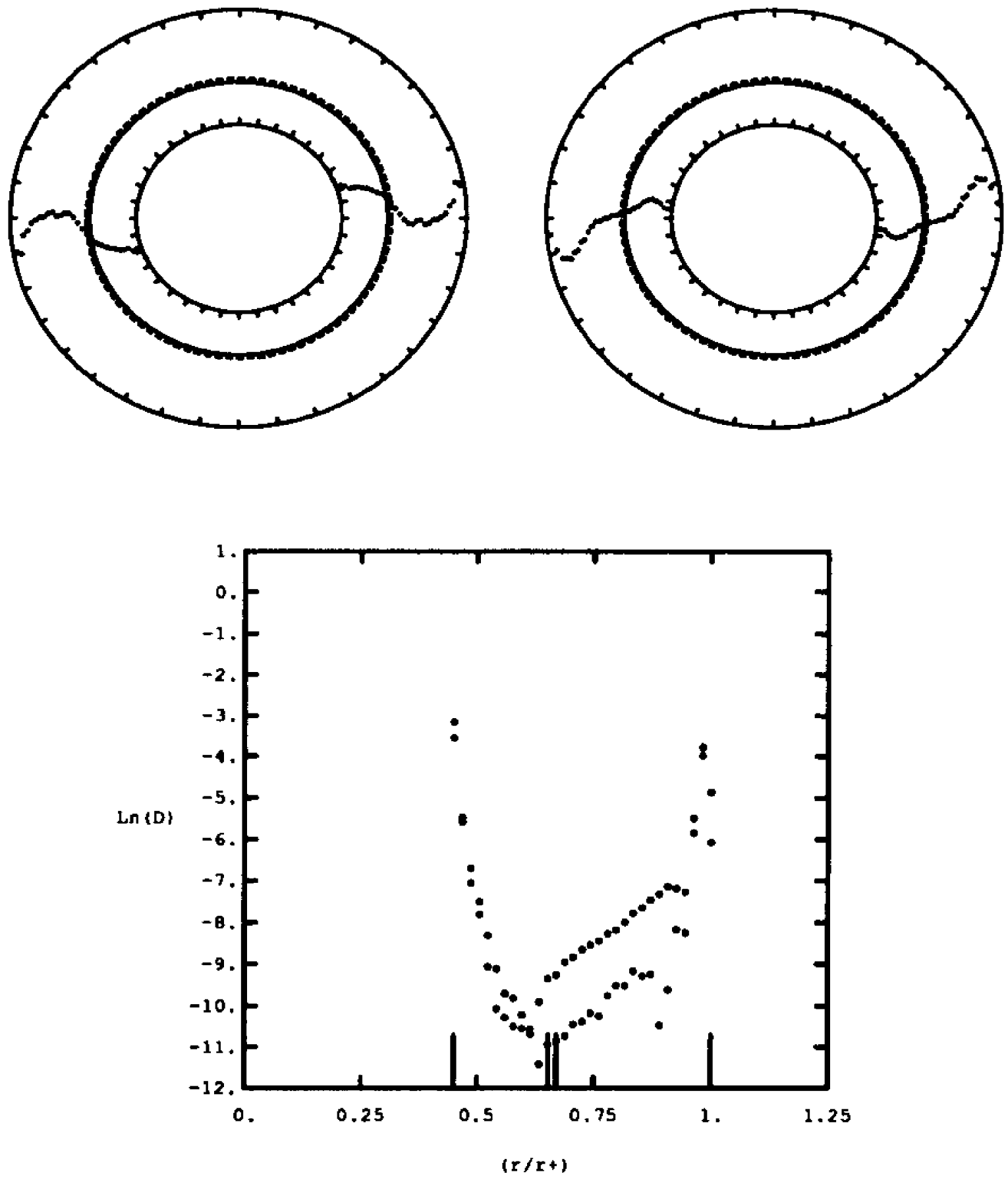

Figure 3.18b: The radial eigenfunction $\left(\phi_{2}-r\right)$ and amplitude as a function of radius $\left(D_{2}-r\right)$ of the $m=2$ Fourier mode illustrated as in Figure 2.3b for $M_{d} / M_{c}=0.2$, model \#3. 

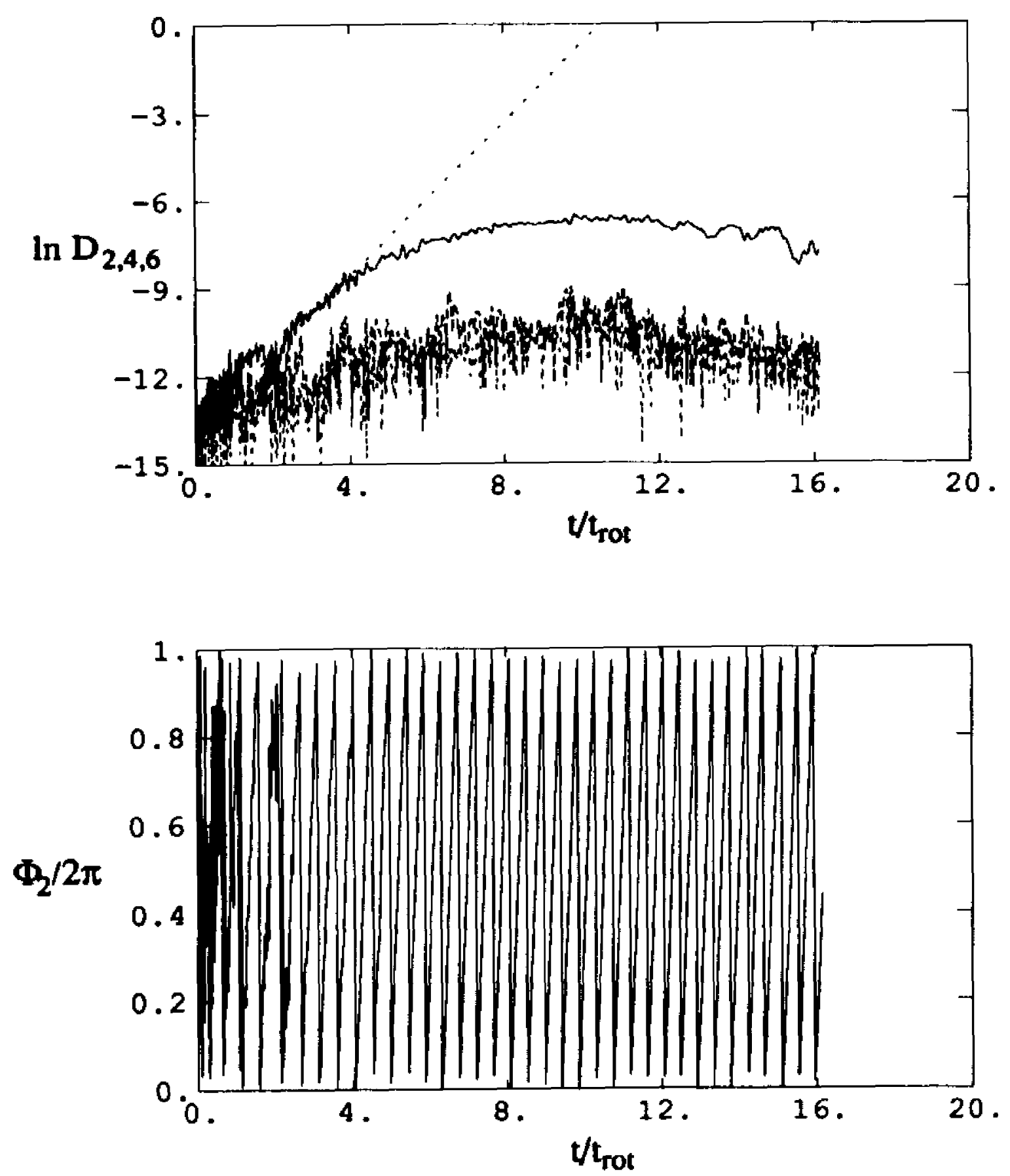

Figure 3.19a: The $m=2, m=4$, and $m=6$ Fourier amplitude behavior, and the $m=2$ phase behavior as a function of time, illustrated as in Figure $2.3 \mathrm{a}$ for $M_{d} / M_{c}=0.2$, model \#4. This model is identified in the text as an ideal example of supercritical stability. 

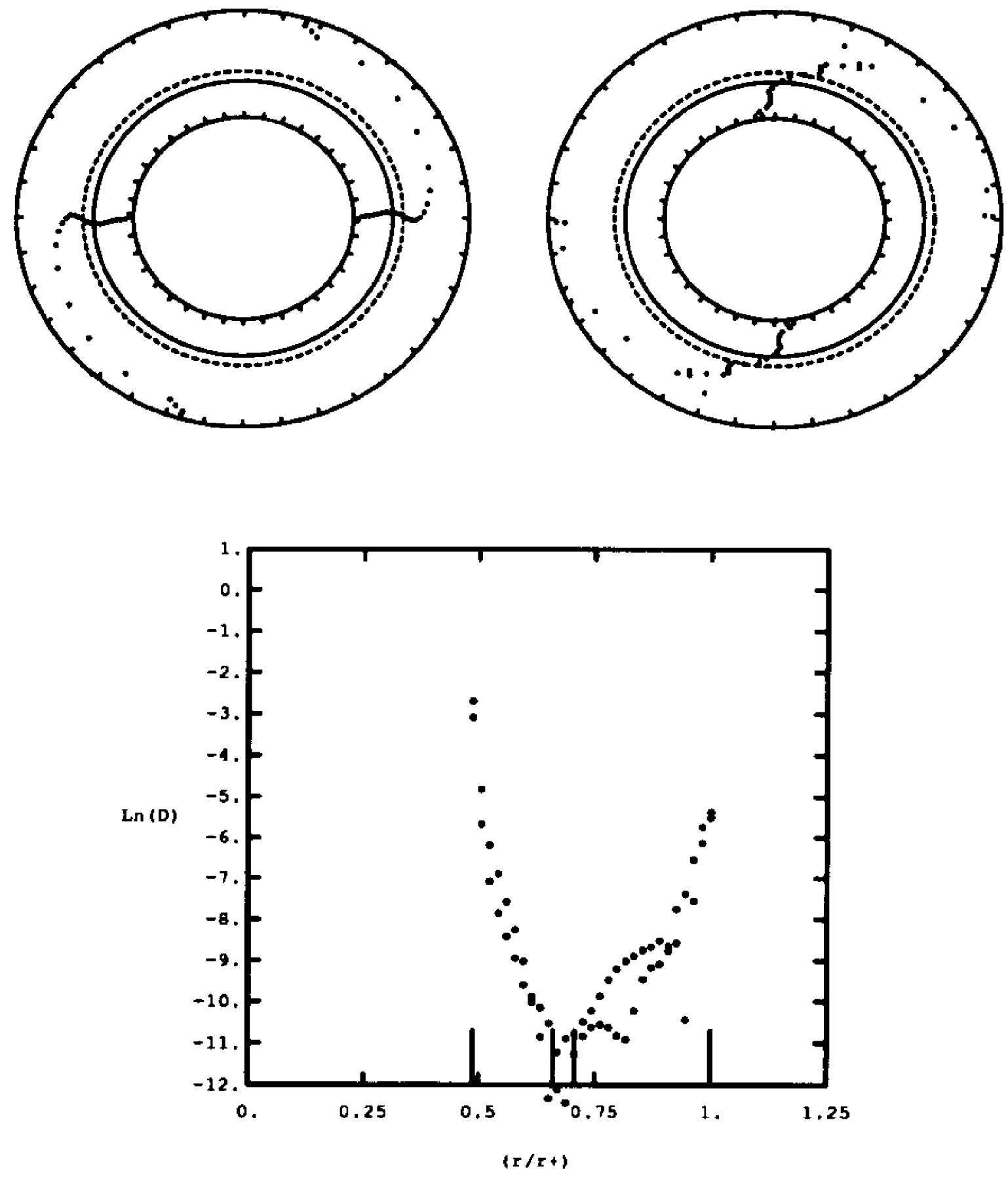

Figure 3.19b: The radial eigenfunction $\left(\phi_{2}-r\right)$ and amplitude as a function of radius $\left(D_{2}-r\right)$ of the $m=2$ Fourier mode illustrated as in Figure $2.3 \mathrm{~b}$ for $M_{d} / M_{c}=0.2$, model \#4. 

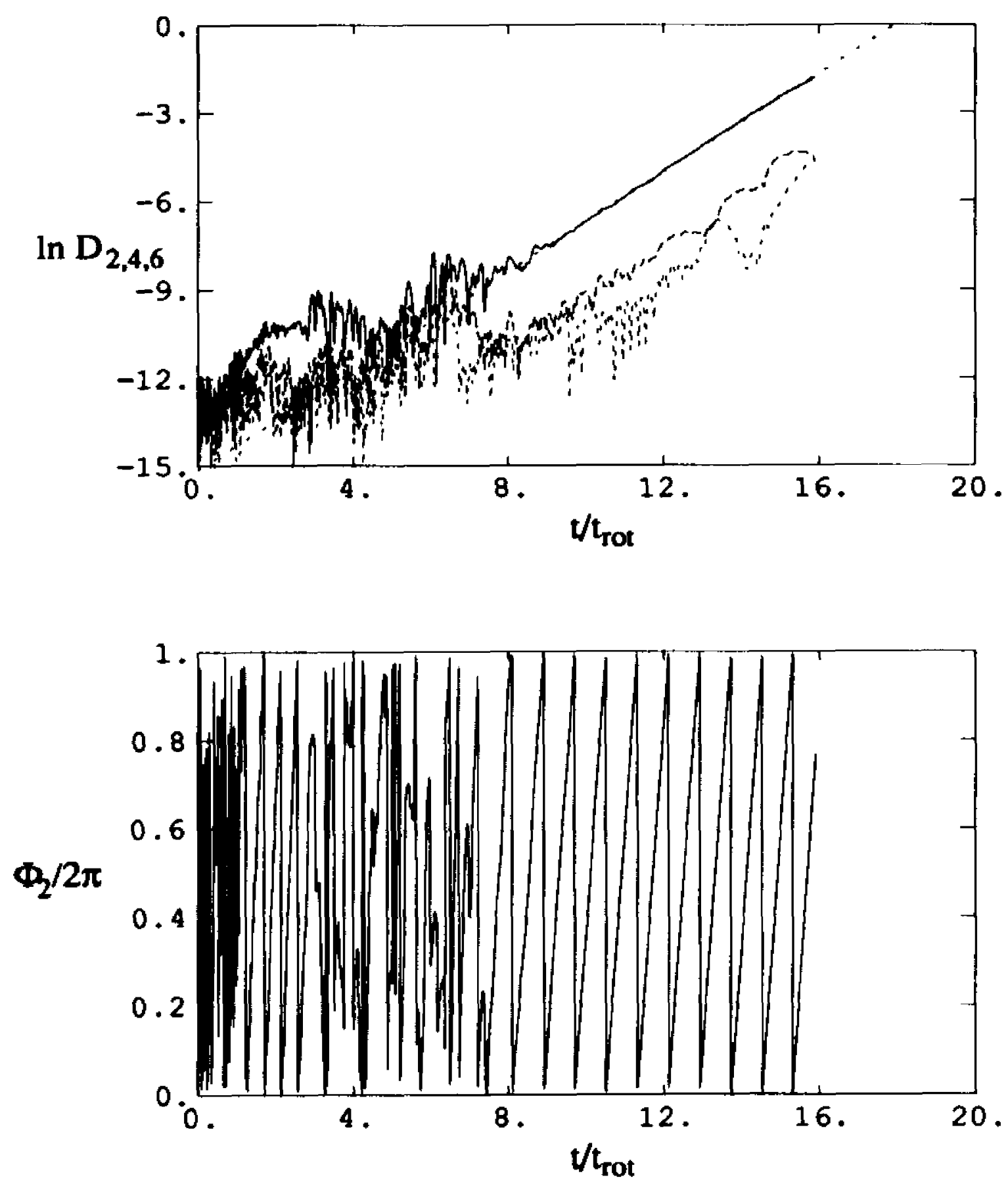

Figure 3.20a: The $m=2, m=4$, and $m=6$ Fourier amplitude behavior, and the $m=2$ phase behavior as a function of time, illustrated as in Figure 2.3a for $M_{d} / M_{c}=0.2$, model \#5. 

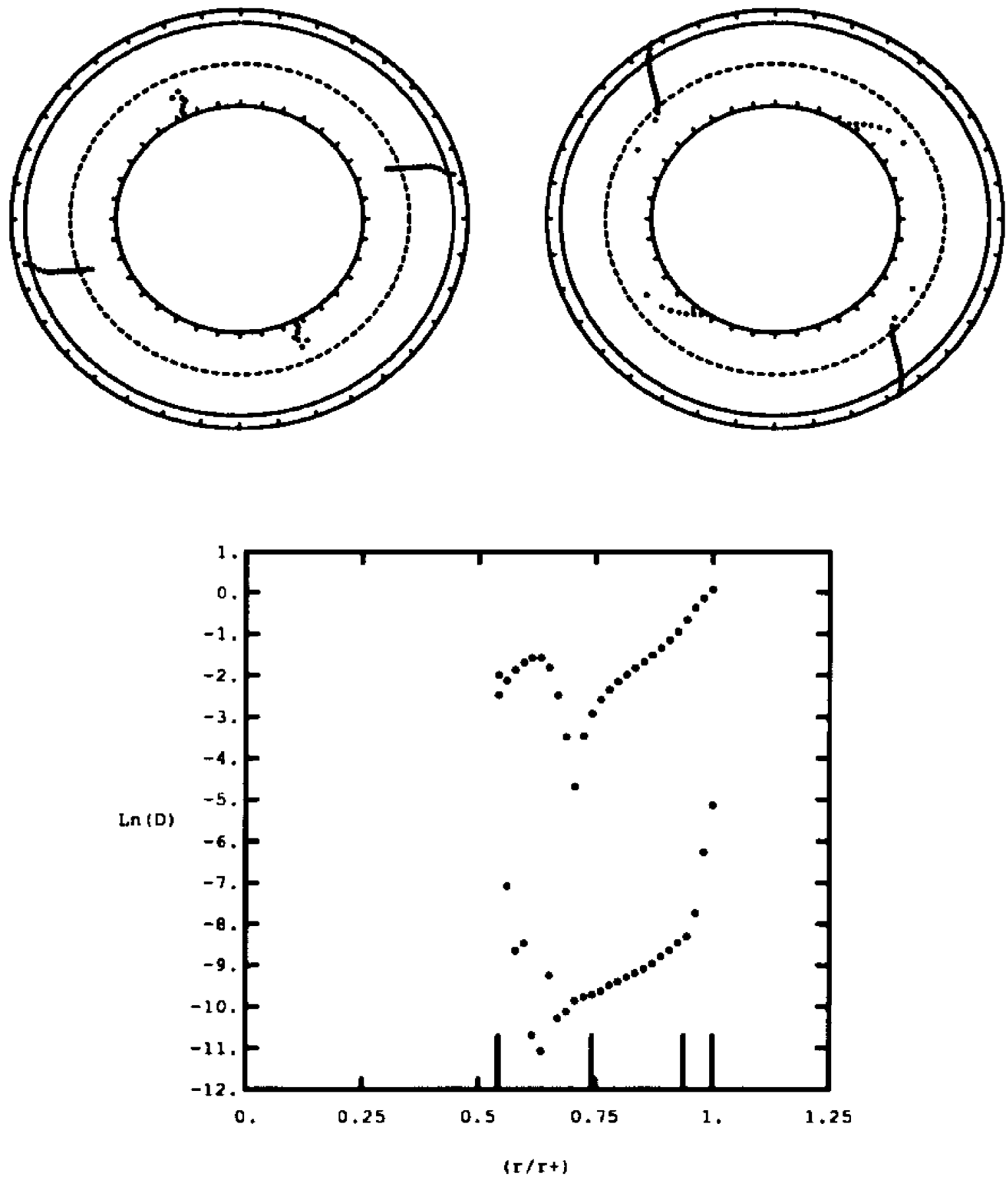

Figure 3.20b: The radial eigenfunction $\left(\phi_{2}-r\right)$ and amplitude as a function of radius $\left(D_{2}-r\right)$ of the $m=2$ Fourier mode illustrated as in Figure 2.3b for $M_{d} / M_{c}=0.2$, model \#5. 

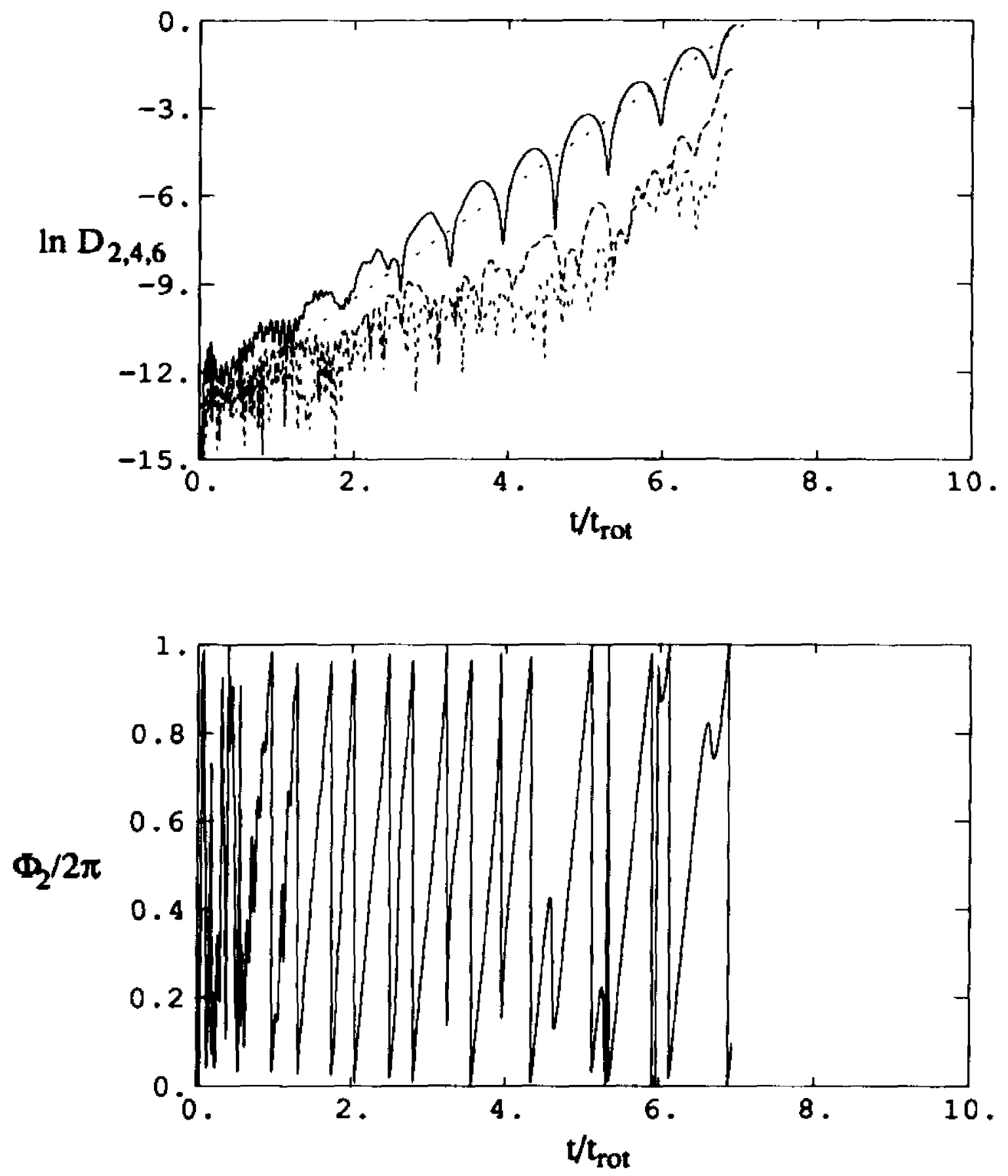

Figure 3.21a: The $m=2, m=4$, and $m=6$ Fourier amplitude behavior, and the $m=2$ phase behavior as a function of time, illustrated as in Figure 2.3a for $M_{d} / M_{c}=0.2$, model \#6. 

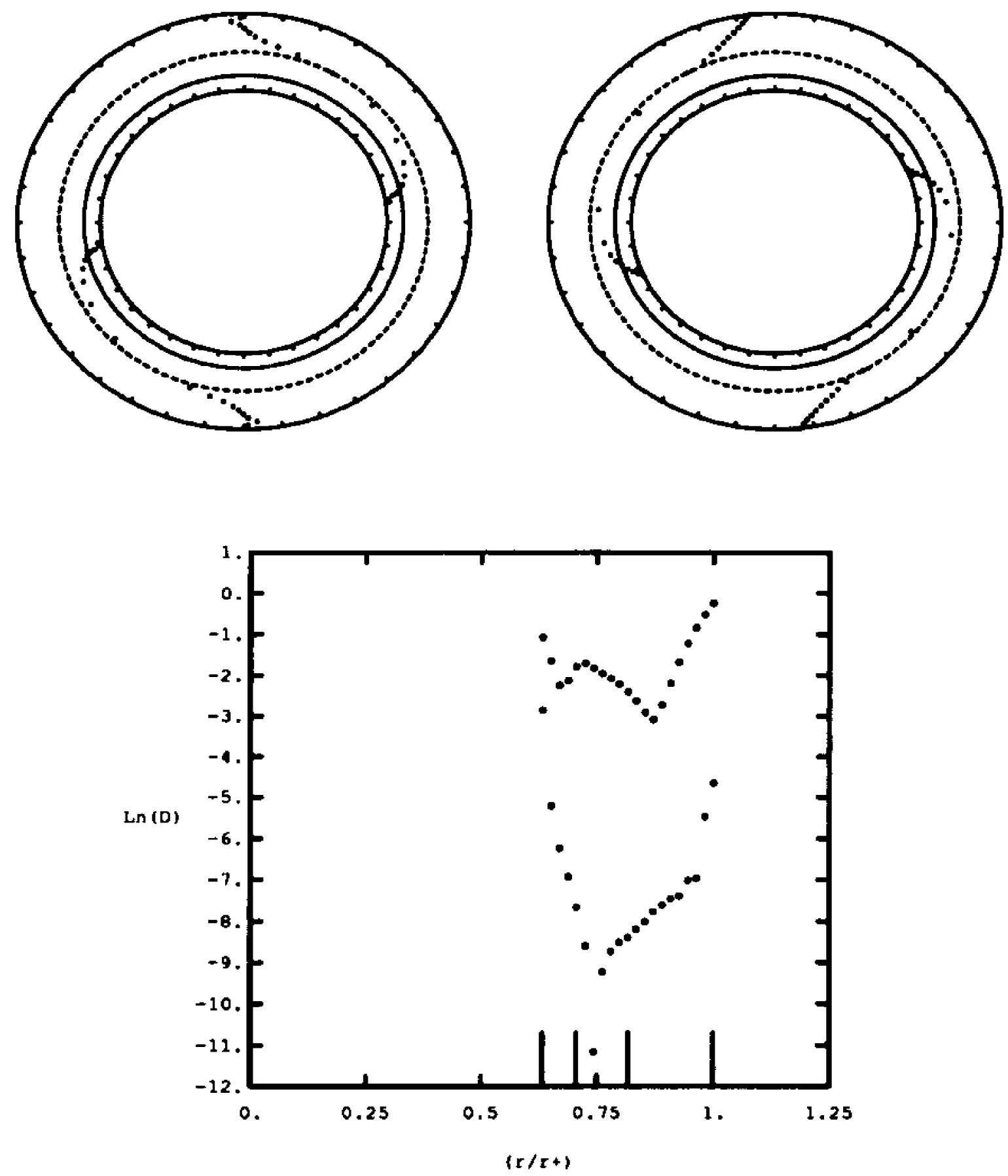

Figure 3.21b: The radial eigenfunction $\left(\phi_{2}-r\right)$ and amplitude as a function of radius $\left(D_{2}-r\right)$ of the $m=2$ Fourier mode illustrated as in Figure 2.3b for $M_{d} / M_{c}=0.2$, model \#6. 

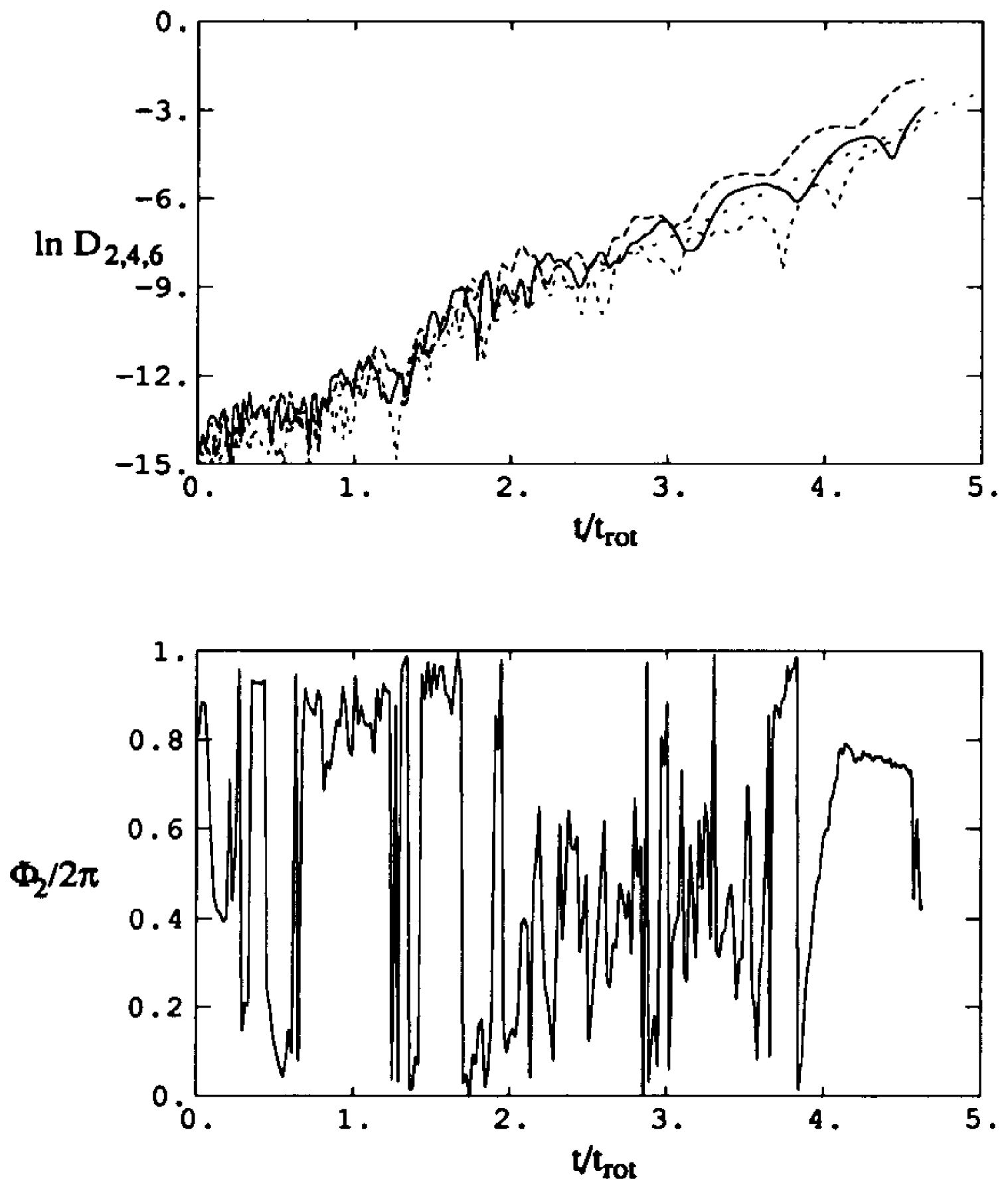

Figure 3.22a: The $m=2, m=4$, and $m=6$ Fourier amplitude behavior, and the $m=2$ phase behavior as a function of time, illustrated as in Figure 2.3a for $M_{d} / M_{c}=0.2$, model \#7. 

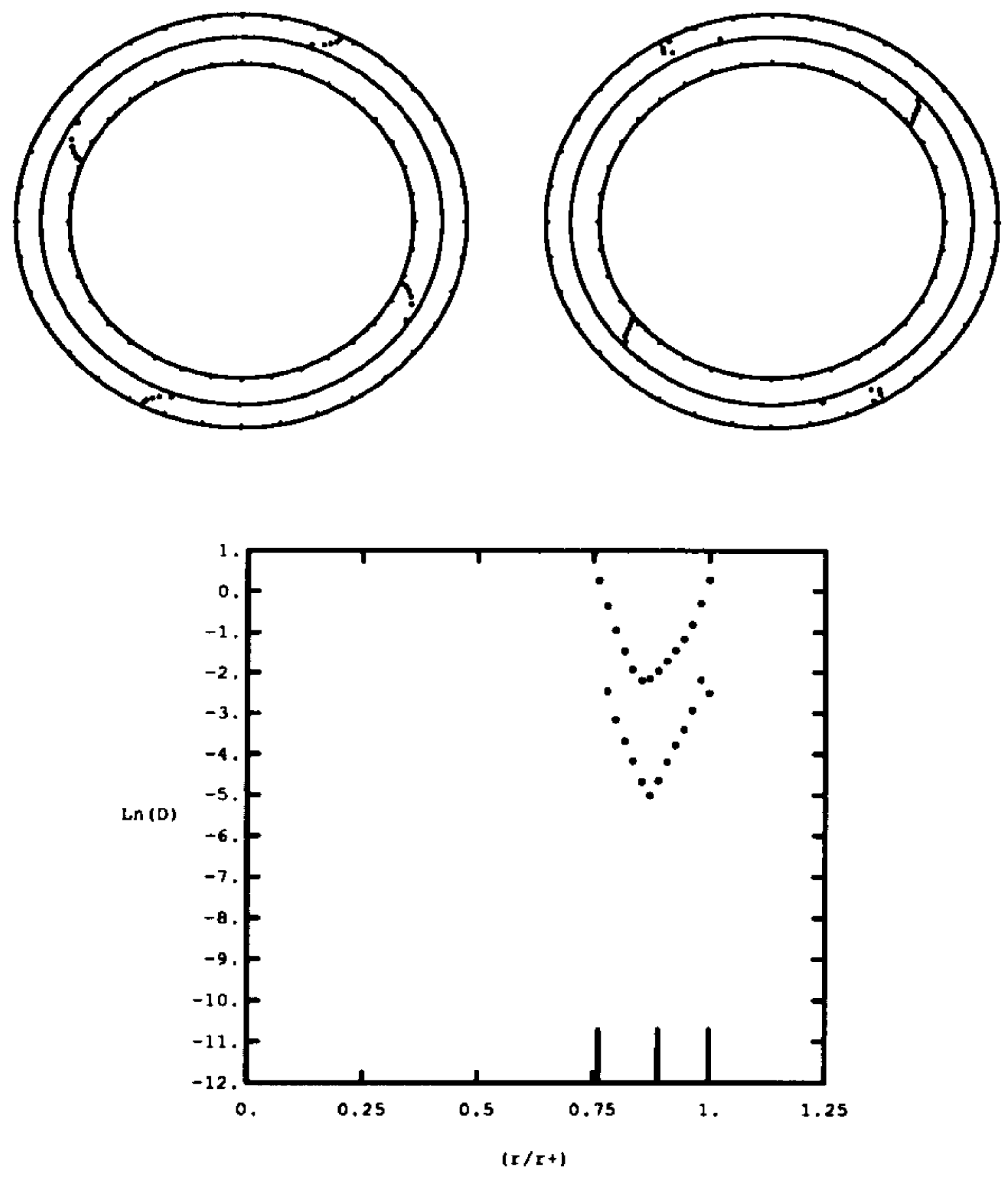

Figure 3.22b: The radial eigenfunction $\left(\phi_{2}-r\right)$ and amplitude as a function of radius $\left(D_{2}-r\right)$ of the $m=2$ Fourier mode illustrated as in Figure 2.3b for $M_{d} / M_{c}=0.2$, model \#7. 


\subsection{Summary of Even-mode Evolutions}

The growth rate and pattern speed parameters $y_{2}(2)$ and $y_{1}(2)$ for all evenmode evolutions have been illustrated in Figure 3.23. Parameters for mass ratios $\infty, 5.0,1.0$, and 0.2 are displayed as crosses, boxes, open circles, and closed circles, respectively.

The principal mode observed in systems with $M_{d} / M_{c}=\infty$ appears to be "ellipsoidal", with a character that is consistent with the I-mode identified by Christodoulou and Narayan (1992):

- $y_{1}(2) \approx-1.0$, indicating a corotation radius that lies outside of $r_{0}$, but inside $r_{+} \cdot$

- $\pi / 2$ phase shift near $r_{0}$.

- $y_{2}$ (2) decreases with decreasing $T /|W|$, dropping to zero at (or near) the marginal stability line as indicated in Figure 3.23.

- Higher order modes develop and eventually dominate if one examines sufficiently high $T /|W|$ (sufficiently "cold") models.

In systems with relatively low mass disks $\left(M_{d} / M_{c}=0.2\right.$ and, perhaps also, 1.0 in the present study), as $T /|W|$ is decreased and the growth rate $y_{2}(2)$ for the principal mode decreases toward zero, competition from another unstable mode is seen. This new unstable mode has the following characteristics:

- $y_{1}(2) \sim+0.1$ to +0.3 and $r_{c r} \approx r_{0}$.

- $\phi_{2}$ vs. $r / r_{0}$ is coherent throughout (no $\pi / 2$ phase shift evident).

- $D_{2}$ vs. $t$ grows early in the evolution, but levels off at a low amplitude, reminiscent of Landau's "supercritical stability" behavior.

To the limits of our ability to measure $y_{2}(1)$, the values of $T /|W|$ for critically unstable models in systems with disk-to-central object mass ratios of 5.0, 1.0, and 
0.2 have been determined to be $0.24,0.34$, and approximately 0.43 , respectively. In Figure 3.24, the $y_{2}(2)$ growth-rate parameter for each model has been illustrated in the $T /|W|$ - mass ratio plane (the diameter of each circle is directly proportional to the measured value of $y_{2}(2)$ ). A smooth curve drawn diagonally across this figure which connects the smallest circle at each mass ratio should fairly represent the demarcation line between systems that are stable and those that are dynamically unstable toward the development of $m=2$ distortions.

The evolutions of these models do not differ significantly from the mass ratio $\infty$ case with the exception of the mass ratio 0.2 (and model \#1 of mass ratio 1.0). Mass ratio 5.0 models showed no significant differences; mass ratio 1.0 models showed the emergence of an oscillatory behavior in the amplitude at high $T /|W|$ in addition to a possible example of supercritical stability at low $T /|W|$; mass ratio 0.2 models showed what appears to be supercritical stability in 3 separate cases. All mass ratios show that as $T /|W|$ is increased, higher order modes become more prominent, eventually having growth rates higher than the lower order modes. This is consistent with general findings of linear theory (Drazin and Reid 1981), the specific linear analysis of zero mass disk systems (Kojima 1986), as well as numerous other studies. 

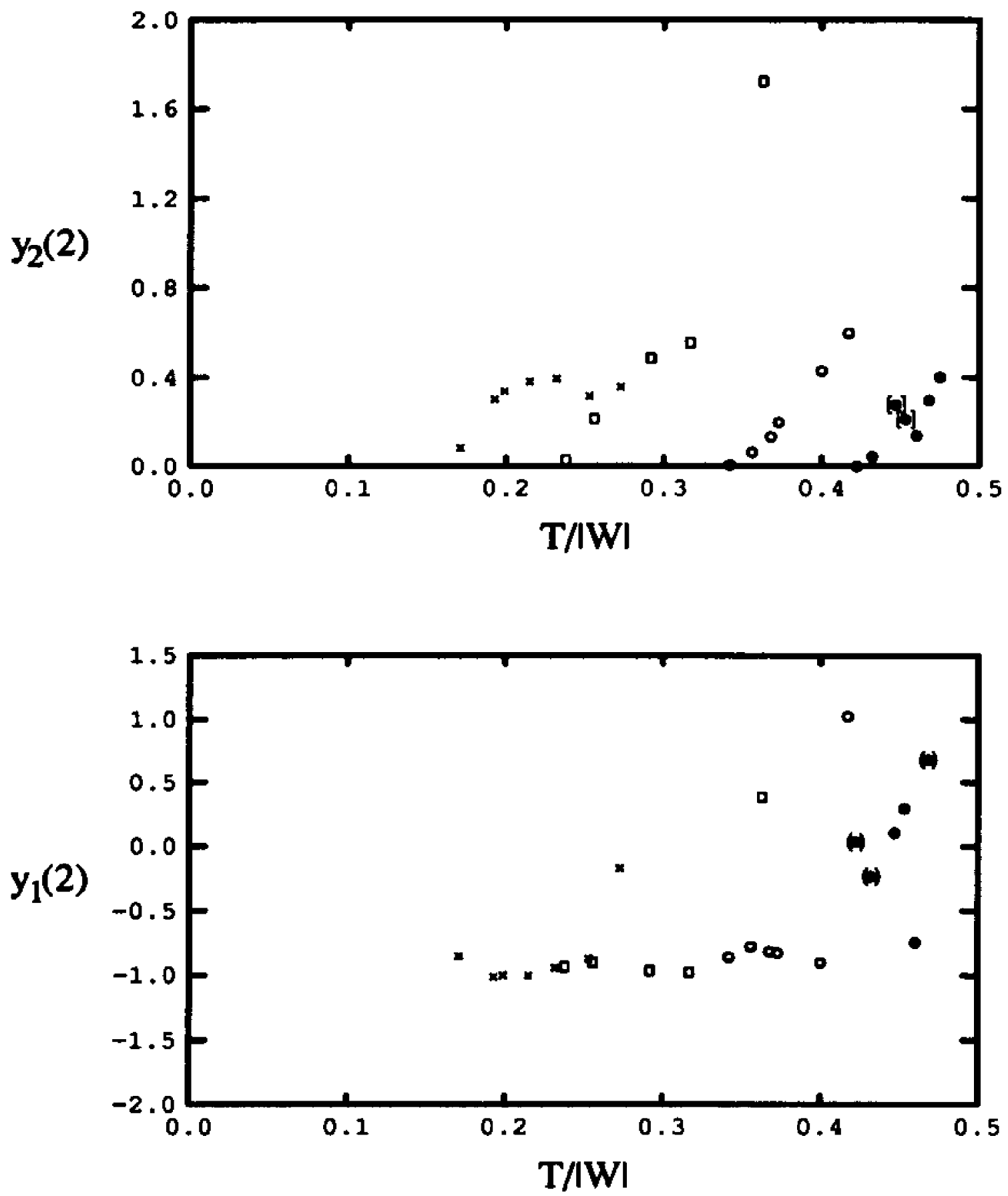

Figure 3.23: $y_{2}(2)$ and $y_{1}(2)$ as functions of $T /|W|$ for all $\pi$-8ymmetry models, mass ratios $M_{d} / M_{c}=\infty$ (crosses), 5.0 (boxes), 1.0 (hollow circles), and 0.2 (solid circles). 


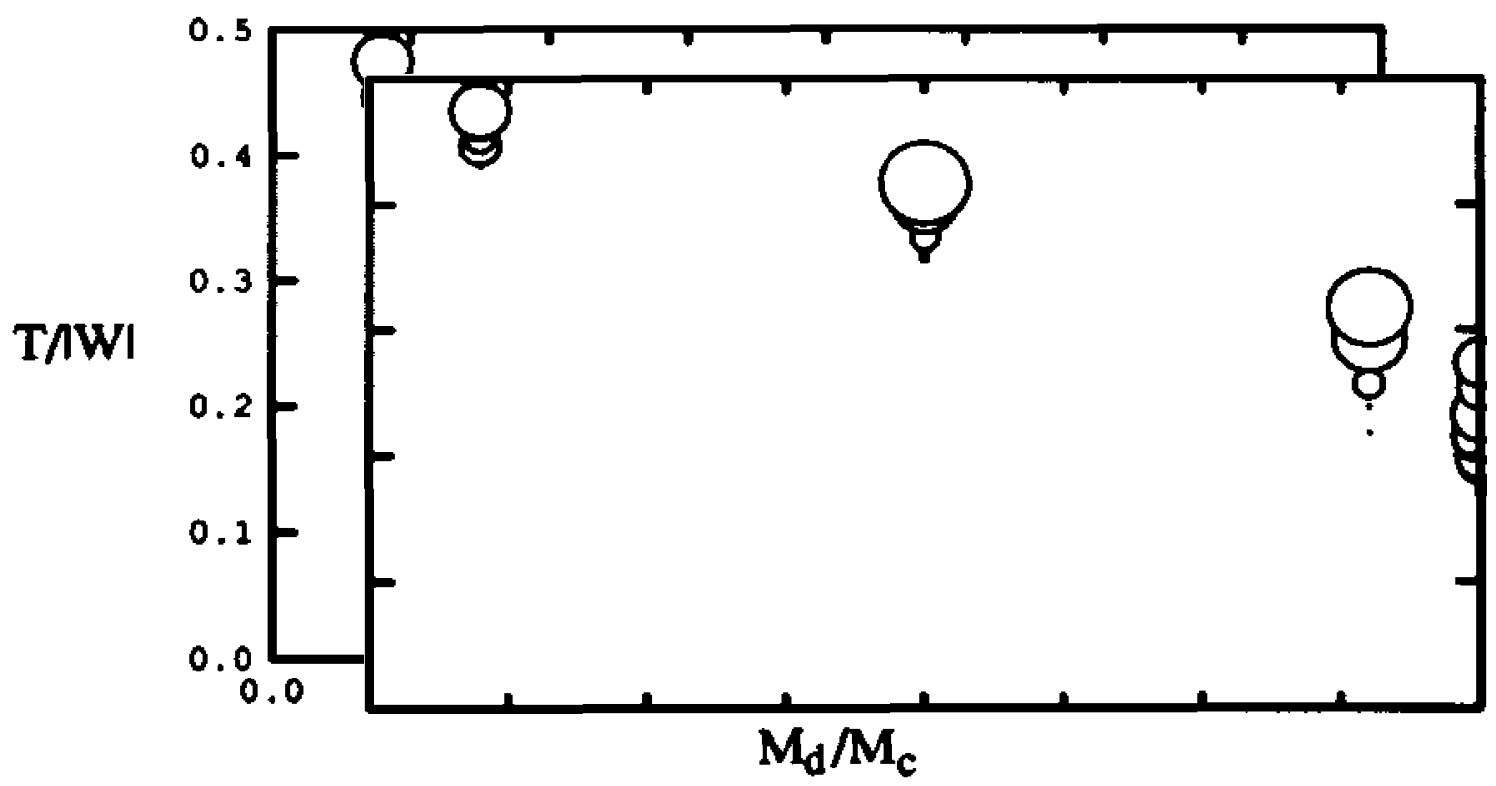

Figure 3.24: Growth-rate results from all the $\pi$-symmetry evolutions are plotted together on a $T /|W|$ vs. $M_{d} / M_{c}$ template. The size of the circles is directly proportional to the growth rate parameter $y_{2}(2)$. 


\section{CHAPTER 4 \\ RESPONSE OF THE CENTRAL OBJECT TO A MASSIVE, AXISYMMETRIC DISK \\ (THE AXISYMMETRIC ROCHE PROBLEM)}

\subsection{Introduction}

In the preceding chapters, little detailed consideration has been given to the central object. Indeed, the central object has been completely neglected except to be considered as a gravitational point source constrained to remain positioned at the center of mass of the system. In this and the following chapter, we consider what types of interactions between the central object and the disk may occur, given that the disk may have a mass comparable to the central object. In this chapter in particular, we examine this interaction between the two components in a somewhat inverted fashion from the usual focus on the stability of the disk. We consider a situation in which the disk, constrained to an axisymmetric configuration, exerts a significant gravitational influence on the central object. Specifically, we are interested in answering the question, "Under what conditions does excretion from the central object occur due to the gravitational influence of the disk?"

\subsection{The Combined Disk, Central-Object Potential}

\subsubsection{Analytic Model}

Throughout most of our analysis in this chapter, we will represent the disk by an infinitesimally thin ring having a finite mass per unit length, and represent the central object by a point mass or, as required, by a spherically symmetric object 
of finite extent. This simple representation permits us to formulate an analytic prescription of the gravitational potential that is not far removed from more realistic - but numerically constructed - equilibrium models of self-gravitating, thick disk aystems such as the ones constructed with the HSCF technique in Chapters 2 and 3 (see detailed comparisons below). By examining the combined gravitational potential of an infinitesimally thin ring and a central point mass, we are, in effect, examining an axisymmetric version of the classical Roche problem for binary stars (Kopal, 1959; Pringle, 1985). This axisymmetric Roche problem apparently has not been fully studied in the published literature, although Wilson (1981) has examined a portion of it.

In preceding chapters, we have symbolized the mass of each extended, dynamically evolving disk by $M_{d}$. In order to emphasize that in the present context the ring is both infinitesimally thin and constrained to remain axisymmetric, we use $M_{R}$ to indicate the mass of the disk/ring. Likewise, in later sections of this chapter, we will discuss our results in terms of central stars having finite radii, not the point masses dealt with in other chapters. Hence, it is useful here to distinguish between the two through the use of $M_{\star}$ rather than $M_{\mathrm{c}}$ to indicate the mass of the central object.

To derive a gravitational potential for the disk, we specifically adopt an axisymmetric ring of radius $r_{0}$. In order to examine when mass will flow between the central object and its surrounding disk, it is necessary only to examine the behavior of the gravitational potential field in the plane of the ring and at radial positions $0<r<r_{0}$. At radij $r<r_{0}$, the potential of the ring can be written either in terms of Legendre polynomials,

$$
\phi_{R}(\alpha)=-\frac{G M_{R}}{r_{0}} \sum_{n=0}^{\infty} \alpha^{2 n}\left[P_{2 n}(0)\right]^{2},
$$


or in terms of the complete elliptic integral of the first kind,

$$
\phi_{R}(\alpha)=-\frac{G M_{R}}{r_{0}} \frac{2}{\pi} K\left(\alpha^{2}\right),
$$

where we have used the definition,

$$
\alpha \equiv r / r_{0}, \quad(0 \leq \alpha<1) .
$$

The potential due to the central object is simply,

$$
\phi_{*}(\alpha)=-\frac{G M_{*}}{r_{0}} \frac{1}{\alpha} .
$$

Defining the dimensionless quantities,

$$
\begin{aligned}
\Phi & \equiv \phi \frac{r_{0}}{G M_{*}}, \\
\mu & \equiv M_{*} / M_{R},
\end{aligned}
$$

the total potential of the system can be written either as

$$
\Phi(\alpha)=-\left\{\frac{1}{\alpha}+\frac{1}{\mu} \sum_{n=0}^{\infty} \alpha^{2 n}\left[P_{2 n}(0)\right]^{2}\right\},
$$

or as

$$
\Phi(\alpha)=-\left\{\frac{1}{\alpha}+\frac{2}{\pi \mu} K\left(\alpha^{2}\right)\right\} .
$$

At some position $\alpha_{1}$ between the central object and the ring, a local maximum will occur in the combined potential. By setting $\partial \Phi / \partial \alpha=0$, the relationship between the position $\alpha_{1}$ and the mass ratio $\mu$ is

$$
\mu=\sum_{n=0}^{\infty} 2 n \alpha_{1}^{2 n+1}\left[P_{2 n}(0)\right]^{2},
$$

or, equivalently,

$$
\mu=\frac{2 \alpha_{1}}{\pi}\left[\frac{E\left(\alpha_{1}^{2}\right)}{1-\alpha_{1}^{2}}-K\left(\alpha_{1}^{2}\right)\right],
$$


Table 4.1

Location of the Potential Maximum

\begin{tabular}{ccc}
\hline \hline$\alpha_{1}$ & $\mu$ & $\log _{10}(\mu)$ \\
\hline 0.0 & $0.000(+0)$ & $-\infty$ \\
0.1 & $5.057(-4)$ & -3.296 \\
0.2 & $4.188(-3)$ & -2.378 \\
0.3 & $1.501(-2)$ & -1.824 \\
0.4 & $3.891(-2)$ & -1.410 \\
0.5 & $8.622(-2)$ & -1.064 \\
0.6 & $1.776(-1)$ & -0.751 \\
0.7 & $3.621(-1)$ & -0.441 \\
0.8 & $7.895(-1)$ & -0.103 \\
0.8266 & $1.000(+0)$ & +0.000 \\
0.9 & $2.227(+0)$ & +0.348 \\
1.0 & $\infty$ & $+\infty$ \\
\hline
\end{tabular}

where the function $E\left(\alpha^{2}\right)$ is the complete elliptic integral of the second kind. Table 4.1 tabulates $\mu$ and $\log \mu$ for a number of representative values of $\alpha_{1}$ and the solid curve in Figure 4.1 displays this relationship graphically. It should be noted that if expression (4.7a) is used to calculate $\mu\left(\alpha_{1}\right)$, a large number of terms ( $n \geq 100$ ) must be included in the series summation in order to get results to the accuracy given in Table 4.1 for $\alpha_{1} \geq 0.8$. Note further that $d \mu / d \alpha_{1}>1$ for all mass ratios $\mu$.

In what follows, we will find it convenient to use $\alpha_{1}$ in place of the independent variable $\mu$. This is allowable because, as Fig. 4.1 illustrates, there is a well-defined one-to-one relationship between the two parameters. We have annotated Fig. 4.1 to 


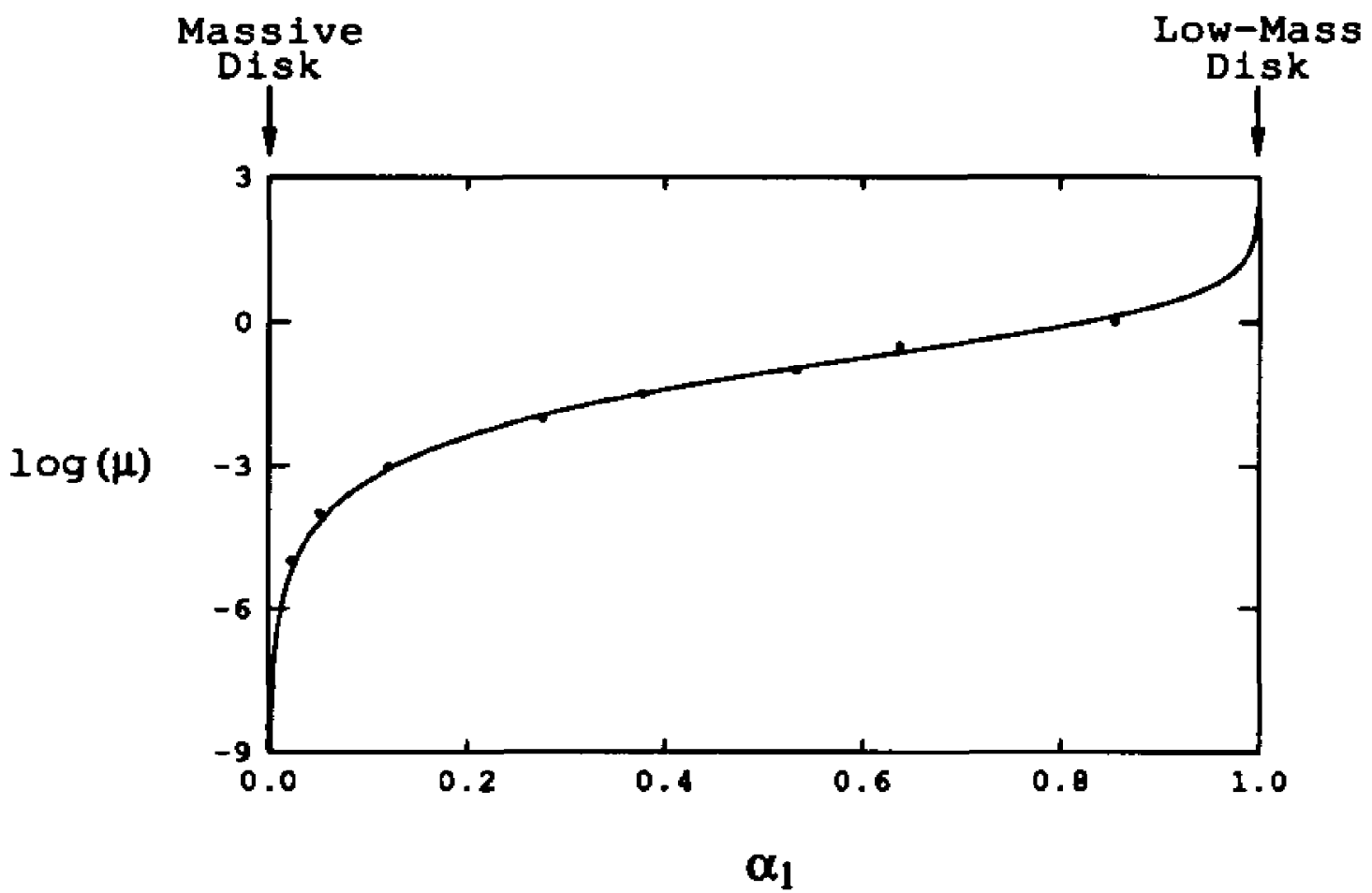

Figure 4.1: The logarithm of the central-object-to-disk mass ratio $\mu$ is plotted versus the radius $\alpha_{1}$ at which a maximum occurs in the combined disk + central object potential. The solid curve shows the smooth behavior prescribed by the analytic relation (4.7); the individual data points show discrete values of $\alpha_{1}$ as determined from thick, equilibrium disk models that have been generated numerically using a self-consistent-field code. As the curve indicates, values of $\alpha_{1}$ near zero correspond to systems with a relatively massive disk; values of $\alpha_{1}$ near unity correspond to systems with a relatively low-mass disk.

emphasize the correlation between $\alpha_{1}$ and $\mu-$ specifically, when $\alpha_{1}$ is near zero we are dealing with a relatively massive ring, while values of $\alpha_{1}$ near unity correspond to systems with a relatively low-mass ring.

\subsubsection{Self-Consistent-Field Model of Thick Disks}

In the above derivation, we have represented the disk by an infinitesimally thin ring primarily because of its mathematical simplicity. Although a thin ring might not seem to be a natural representation of low-mass disks that have Keplerian 


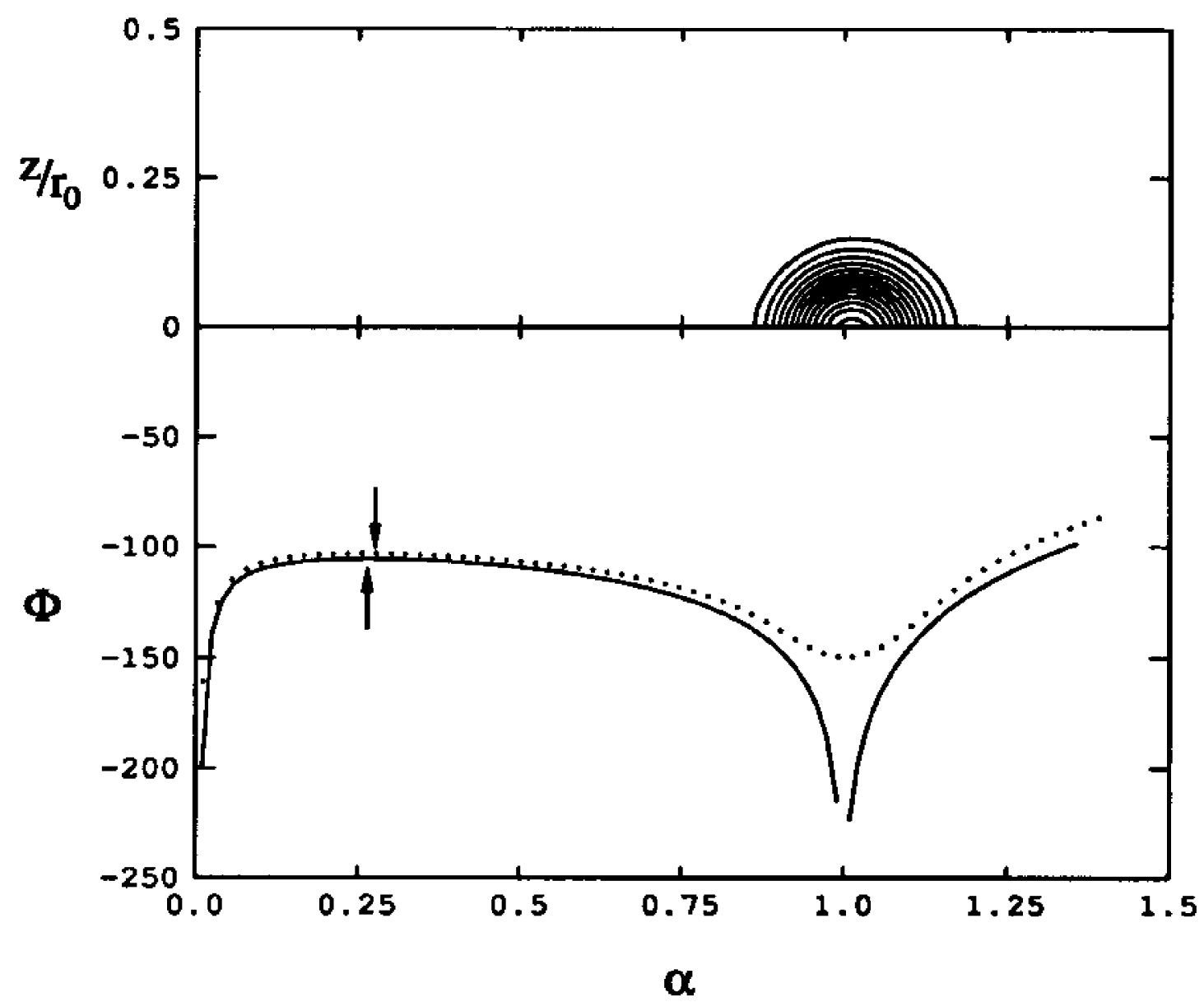

Figure 4.2: The top panel displays a meridional cross-section through an $n=3 / 2$ polytropic disk that is 100 times more massive than the central object (not shown) which it surrounds. This numerically constructed equilibrium disk has a Keplerian velocity profile and a ratio of rotational to gravitational potential energy $T /|W|=0.331$. The bottom panel displays the radial behavior of the gravitational potential in the equatorial plane of this $\mu=10^{-2}$ system as derived from the numerical model (dotted curve), and as prescribed analytically (solid curve). The spacing of the points along the dotted curve indicates the spatial resolution of the numerical model. The upper (lower) arrow indicates the position of $\alpha_{1}$ for the numerical (analytical) model.

rotation curves, equation (4.6) proves to be an accurate representation of the combined disk plus central-object potential in thick disk systems, particularly in situations where the disk contains more mass than does the central object. This is 
in part because equilibrium models of such disks often look like tori. In order to demonstrate this, we have used Andalib's (1992) HSCF code to construct detailed force-balanced models of thick, axisymmetric accretion disks having a variety of disk-to-central-object mass ratios and have compared the radial potential functions derived numerically for these systems to the potential function prescribed analytically by equation (4.6). Figure 4.2 illustrates one such comparison.

The top panel of Figure 4.2 displays a meridional cross-section through a polytropic disk $(n=3 / 2)$ that has a Keplerian rotation curve $(q=3 / 2)$, a ratio of rotational to gravitational potential energy $T /|W|=0.331$ and a disk-to-centralobject mass ratio of 100.0 (i.e., $\mu=10^{-2}$ ). The bottom panel of Figure 4.2 displays the gravitational potential as a function of radius in the equatorial plane of this system as derived numerically from the self-consistent-field code (dotted curve) and as prescribed analytically by equation (4.6) for $\mu=10^{-2}$ (solid curve). The only significant difference between the two potential curves occurs in the vicinity of $\alpha=1.0$ where the more realistic, numerically constructed model avoids the mathematical singularity encountered in the analytic model.

Of principal concern to us here is the radial location $\alpha_{1}$ of the maximum in the potential function. The arrows in the bottom panel of Figure 4.2 identify the location of $\alpha_{1}$ as determined by the two separate methods (the value determined numerically is slightly larger than the value determined analytically). The numerically determined value of $\alpha_{1}$ is also identified by the data point located at $\log (\mu)=-2$ in Figure 4.1. The other data points in Figure 4.1 identify values of $\alpha_{1}$ that we have derived numerically in a similar manner for systems with other disk-to-central-object mass ratios. (Because we are focusing, in this chapter, on systems in which the disk makes a non-negligible contribution to the gravitational potential, only systems in which the disk has a mass greater than or equal to the mass of the central object 
are identified.) In general, the agreement between our simple analytic model and the more realistic, numerically generated ones is very good. This gives us confidence that stability conditions derived using the analytic potential function given in equation (4.6) will be meaningful in realistic astrophysical settings.

\subsection{The Condition for Runaway Excretion}

In order to address the question of central object excretion, it is necessary to give the central object a finite radius $r_{*}$ or, relative to the size of the ring, a dimensionless radius $\alpha_{\star} \equiv r_{\star} / r_{0}$. If the central object is rotating, the task of specifying $r_{*}$ is a nontrivial one. For simplicity, we will assume that the central object is nonrotating and is spherically symmetric, so the expressions derived above will properly describe the interaction potential as long as the discussion is restricted to radii $\alpha_{*} \leq \alpha<1$. Also, as long as we restrict the discussion to excretion from a nonrotating central object, we need not modify the combined potential to include the effects of rotation. In this sense, our problem differs significantly from the binary Roche problem (Kopal, 1959; Pringle, 1985).

Although we are not including rotation or orbital motion in this problem, an analogy can be drawn between the radial position $\alpha_{1}$ and the position of the $L_{1}$ Lagrange point in the classical Roche problem. Because we are dealing with an axisymmetric system, however, the position $\alpha_{l}$ is not associated with a single point. Instead, it identifies a locus of points lying on a circle that is concentric with the ring. If, for a given mass ratio $\mu$, the central object's radius $\alpha_{\star}>\alpha_{1}$, then material will flow away from its surface, radially outward in the plane of the ring. On a dynamical time scale, the central object will excrete material axisymmetrically; the material will, in turn, accrete onto the ring (or disk), hitting its inner edge first. 
Will this axisymmetric excretion be a runaway process, effectively destroying the central object on a dynamical time scale? The answer to this question depends on the mass-radius relation of the central object $-i . e .$, on the derivative $d \alpha_{\star} / d M_{\star}$ - and on the derivative $d a_{1} / d M_{*}$. Specifically, the condition for runaway is

$$
\frac{d \alpha_{\star}}{d M_{\star}} \leq \frac{d \alpha_{1}}{d M_{\star}}
$$

If the total system mass,

$$
M_{T o T} \equiv M_{R}(1+\mu),
$$

and the ring radius $r_{0}$ are held fixed during the excretion process, then

$$
\frac{d \alpha_{1}}{d M_{*}}=\frac{(1+\mu)^{2}}{M_{T O T}}\left[\frac{d \mu}{d \alpha_{1}}\right]^{-1} .
$$

If, furthermore, we characterize the central object's mass-radius relation by the power-law

$$
r_{*}=k M_{*}^{*}
$$

where $k$ is a constant, then the condition for runaway excretion becomes

$$
\nu \leq \nu_{\text {crit }} \equiv \frac{\mu(1+\mu)}{\alpha_{1}}\left[\frac{d \mu}{d \alpha_{1}}\right]^{-1} .
$$

In deriving $\nu_{\text {crie }}$, we have set $\alpha_{\star}=\alpha_{1}$, which is the condition that will hold at the onset of mass transfer. Using equations (4.7a) and (4.7b), respectively, we derive

$$
\begin{aligned}
\nu_{\text {crit }}\left(\alpha_{1}\right) & =\frac{\mu(1+\mu)}{\sum_{n=0}^{\infty} 2 n(2 n+1) \alpha_{1}^{2 n+1}\left[P_{2 n}(0)\right]^{2}} \\
& =\frac{\mu(1+\mu)}{\alpha_{1}}\left[\frac{\pi\left(1-\alpha_{1}^{2}\right)}{2}\right]\left[\frac{1+\alpha_{1}^{2}}{1-\alpha_{1}^{2}} E\left(\alpha_{1}^{2}\right)-K\left(\alpha_{1}^{2}\right)\right]^{-1} .
\end{aligned}
$$

In expressions (4.13a) and (4.13b), $\mu$ is implicitly assumed to have the functional dependence on $\alpha_{1}$ given by expression (4.7a) or (4.7b). The solid curve in Figure 4.3 graphically displays the function $\nu_{\text {crit }}\left(\alpha_{1}\right)$. 


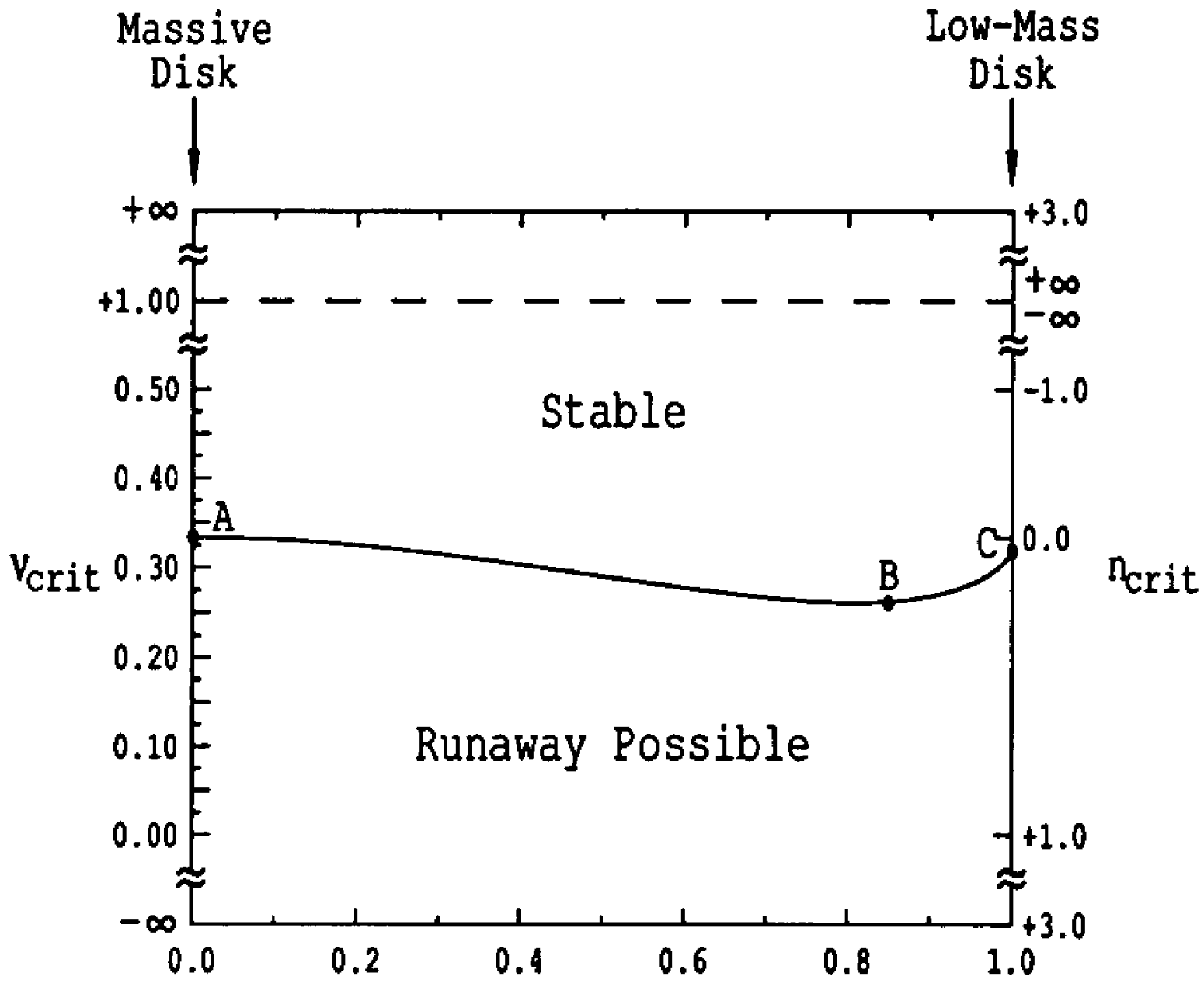

$\alpha_{1}$

Figure 4.3: The solid curve in this graph displays the function $\nu_{\text {erit }}\left(a_{1}\right)$ given by equation (4.13). Central objects with power-law mass-radius relations in the exponent range $\nu \geq \nu_{c r i t}(A)=1 / 3$ are always stable against runaway excretion. Once excretion begins, central objects having $\nu \leq \nu_{\text {crit }}(B)=0.261$ will always encounter runaway excretion. For central objects with $\nu_{\text {cril }}(B)<\nu<\nu_{\text {cril }}(A)$, whether or not runaway excretion occurs depends upon the mass ratio of the system at the onset of excretion. The critical polytropic index $n_{\text {crit }}$ that corresponds to each exponent $\nu_{\text {crit }}$ is identified along the right-hand vertical axis. 
Table 4.2

Special Points in Figure 4.3

\begin{tabular}{cccccc}
\hline \hline Point & $\alpha_{1}$ & $\mu$ & Significance & $\nu_{\text {erit }}$ & $n_{\text {crit }}$ \\
\hline A & 0 & 0 & $M_{*}=0$ & $1 / 3$ & 0 \\
B & 0.808 & 0.849 & $\frac{d \nu_{\text {sin }}}{d \alpha_{1}}=0$ & 0.261 & 0.292 \\
C & 1 & $+\infty$ & $M_{R}=0$ & $1 / \pi$ & $\frac{\pi-3}{\pi-1}=0.066$ \\
\hline
\end{tabular}

Because spherical polytropic stars exhibit power-law mass-radius relations, there is a one-to-one relationship between $\nu$ and the polytropic index $n$ :

$$
n=(3 \nu-1) /(\nu-1)
$$

Based on this relation, the right-hand vertical scale in Fig. 4.3 identifies the critical polytropic index $n_{\text {crit }}$ that corresponds to each $\nu_{\text {crit }}$.

Figures 4.1 and 4.3 can now be used in conjunction with one another in order to determine the condition for runaway excretion in any system. For a given ratio $\mu$, the curve in Fig. 4.1 identifies the radius that the central object must have before mass excretion will begin. If this critical size is realized, then $\alpha_{\star}=\alpha_{1}$ and Fig. 4.3 identifies $\nu_{\text {crit }}$, a critical exponent for the central object's mass-radius relation. Three special points arise along the curve in Figure 4.3 - points $A, B$, and $C$. Their values and respective meanings are tabulated in Table 4.2. (NOTE: In the limit $\alpha_{1} \rightarrow 1$, i.e., at point $C$, we have used equation (4.13b) in conjunction with the tools of Mathematica to demonstrate that $\nu_{\text {erit }} \rightarrow 1 / \pi$, exactly.) Also listed in Table 4.2 are the critical polytropic indices that correspond to points $A$, $B$, and $C$. Based on these special points, Figure 4.3 can be divided vertically into three physically distinct regions. 
Region I - Independent of the mass ratio $\mu$, a star exhibiting a structural

$$
\nu \geq \nu_{\text {crit }}(A)=1 / 3
$$

will never encounter runaway excretion. As the right-hand scale of Fig. 4.3 indicates, only stars with negative polytropic indices or with $n \geq+3$ fall into this region.

Region II - Once the excretion process starts, a star having a structural

$$
\nu \leq \nu_{\text {crit }}(B)=0.261
$$

will always encounter runaway excretion, independent of $\mu$. Stars having polytropic indices $0.292 \leq n<3-$ for example, fully convective protostars - fall into this region.

Region III - For mass-radius relations exhibiting

$$
\nu_{\text {cril }}(B)<\nu<\nu_{\text {cril }}(A)
$$

whether runaway excretion will occur or not depends on the mass ratio of the system when excretion begins. Consider, for example, a star having a structural $\boldsymbol{\nu}=\mathbf{0 . 3}$ (i.e., an $n=0.143$ polytrope). According to Fig. 4.3, runaway excretion will occur only if $\alpha_{1}<0.426-$ that is, via. Fig. 4.1 , only if $\mu<0.048$ initially.

\subsection{Discussion}

Based on the preceding derivations, we conclude that material will be tidally stripped from the surface of a star if an axisymmetric disk surrounding the star is sufficiently massive and if the star's radius is a sufficiently large fraction of the surrounding disk's radius. For the entire range of star-to-disk mass ratios, $0 \leq \mu \leq$ $\infty$, we have determined the critical stellar radius $\alpha_{1}$ above which tidal stripping will 
occur in the plane of the disk. The function $\mu\left(\alpha_{1}\right)$ is given analytically by equation (4.7a) or (4.7b) and is displayed graphically in Figure 4.1.

We have identified the physical conditions under which runaway excretion will occur from a nonrotating, spherically symmetric central star once tidal stripping has been initiated. Runaway excretion will always occur if the central star has a polytropic index $3>n>0.292$ or, more generally, if the star exhibits a massradius relation equivalent to a polytropic star that has an index in this range. If the central star has a polytropic index (or an equivalent mass-radius relationship) in the range $0.292>n>0$, runaway excretion will occur only if the surrounding disk is sufficiently massive at the onset of tidal stripping. The minimum disk mass required varies with the polytropic index of the central star but, as tabulated in Table 4.2, in this range of polytropic indices the star-to-disk mass ratio can be no greater than 0.849 in order for runaway excretion to occur. Tidal stripping will not lead to runaway excretion if the central star has a negative polytropic index or if it's equivalent polytropic index falls in the range $\infty \geq n \geq 3$, but these indices lie outside the regime commonly considered appropriate for normal stars. Hence, we have demonstrated that, at least in principle, realistic central stars can be disrupted gravitationally in the presence of a sufficiently massive diak.

In what astrophysical settings is the tidal disruption of a central star by a disk actually going to occur? Because protostars exhibit mass-radius relations that fall into the "Region II" category described above (i.e., $3>n>0.292$ ), the condition for complete disruption reduces simply to the condition for the onset of tidal stripping,

$$
\alpha_{\star} \geq \alpha_{1}
$$

Invoking global equilibrium arguments, this condition can be transformed into a conditional relationship between the orbital velocity of the disk material and the 
mean sound speed of material in the central star. Virial equilibrium ensures that the mean sound speed $c_{\star}$ of the material in the nonrotating central star is

$$
c_{*}^{2} \sim \frac{G M_{*}}{r_{*}}
$$

Similarly, in order to achieve equilibrium in the disk plus central star combined gravitational field, the rotational velocity of the disk material must be

$$
v_{R}^{2} \sim \frac{G M_{T O T}}{r_{0}} .
$$

Combining eqs. (4.19) and (4.20), then,

$$
\alpha_{*} \sim \frac{M_{*}}{M_{T O T}}\left(\frac{v_{R}}{c_{*}}\right)^{2}=\frac{\mu}{(1+\mu)}\left(\frac{v_{R}}{c_{*}}\right)^{2},
$$

and condition (4.18) becomes, approximately,

$$
\left(\frac{v_{n}}{c_{*}}\right)^{2} \gtrsim \alpha_{1}\left(1+\frac{1}{\mu}\right) \text {. }
$$

In order to illustrate the magnitude of this condition, $\log (\mu)$ has been plotted versus the quantity $\log \left[\alpha_{1}(1+1 / \mu)\right]$ in Fig. 4.4.

Up to this point, our formalism has permitted us to consider all possible values of the star-to-disk mass ratio $\mu$. Realistically, however, the disk is not likely to perturb the central star siguificantly unless it contains at least as much mass as the star, i.e., unless $\mu \lesssim 1.0$. Invuking glubal stability arguments in this regime, we can further transform relation (4.22) into a conditional relationship between $\tau_{R}$, the mean temperature of the disk material, and $\tau_{*}$, the mean temperature of material in the central star. Generally, the ratio $f$ of thermal energy to rotational kinetic energy in the disk can be writteu as

$$
f \approx c_{R}^{2} / v_{R}^{2}
$$




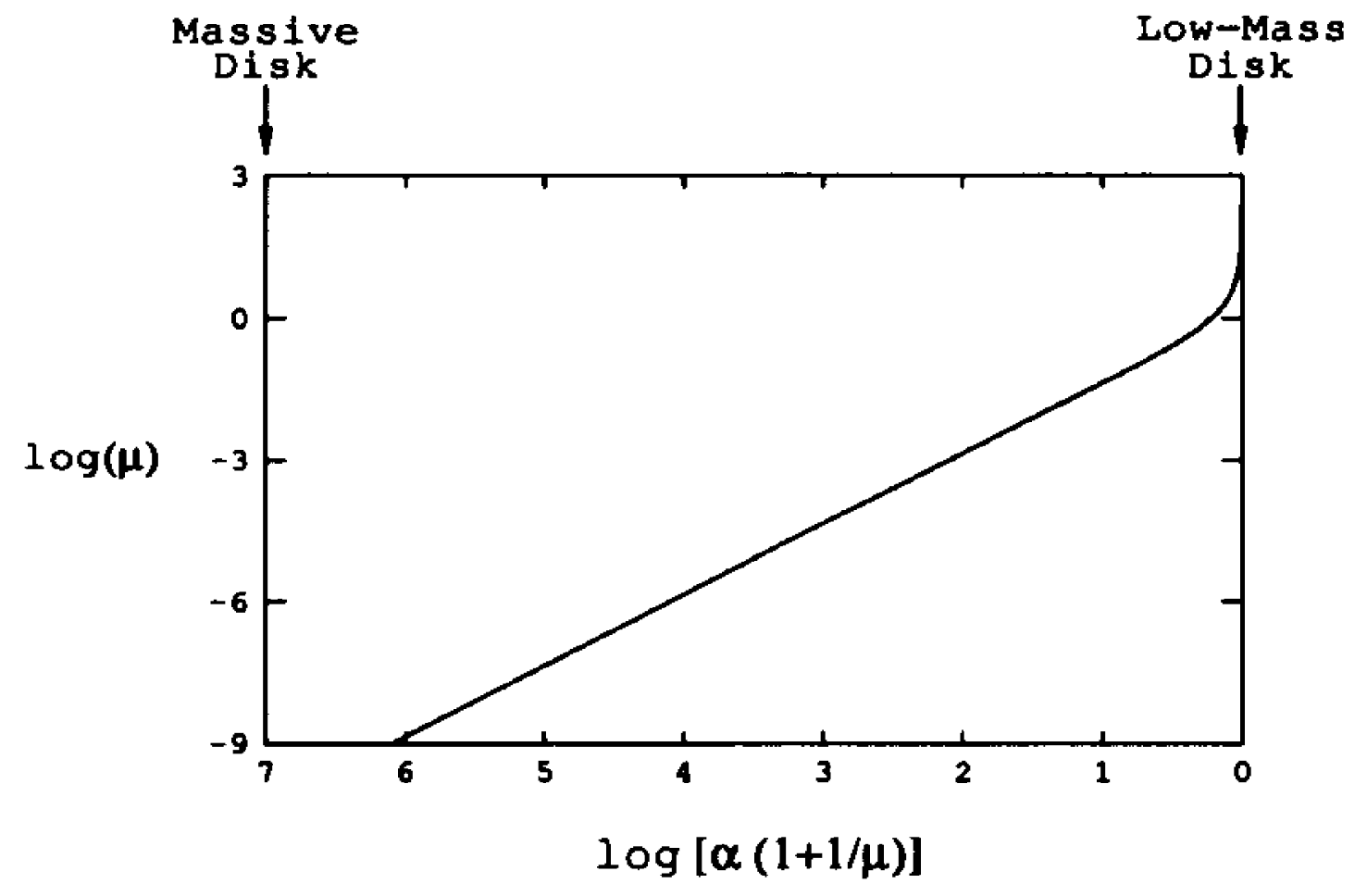

Figure 4.4: The logarithm of the central-object-to-disk mass ratio $\mu$ is plotted versus the quantity $\log \left[\alpha_{1}(1+1 / \mu)\right]$ to illustrate the behavior of relation (4.22). In order to simplify comparisons between this diagram and earlier figures in the text, the abscissa has been plotted with values increasing to the left.

where $c_{R}$ is the mean sound speed in the disk. As has been demonstrated in Chapters 2 and 3, in order for a self-gravitating disk to be stable against both axisymmetric and nonaxisymmetric, dynamical instabilities, the disk must be sufficiently hot; that is, $f$ must be greater than some critical value. In the case where no central object is present, i.e., when $\mu=0$, we showed in Chapter 2 that an axisymmetric ring or disk will be dynamically stable only if $f \gtrsim 2$. For $\mu \sim 1$, the lower limit on $f$ set by our stability analysis drops somewhat, but it remains of order unity. Because the disk's own dynamical stability is ensured only when $f \sim 1$ in systems 


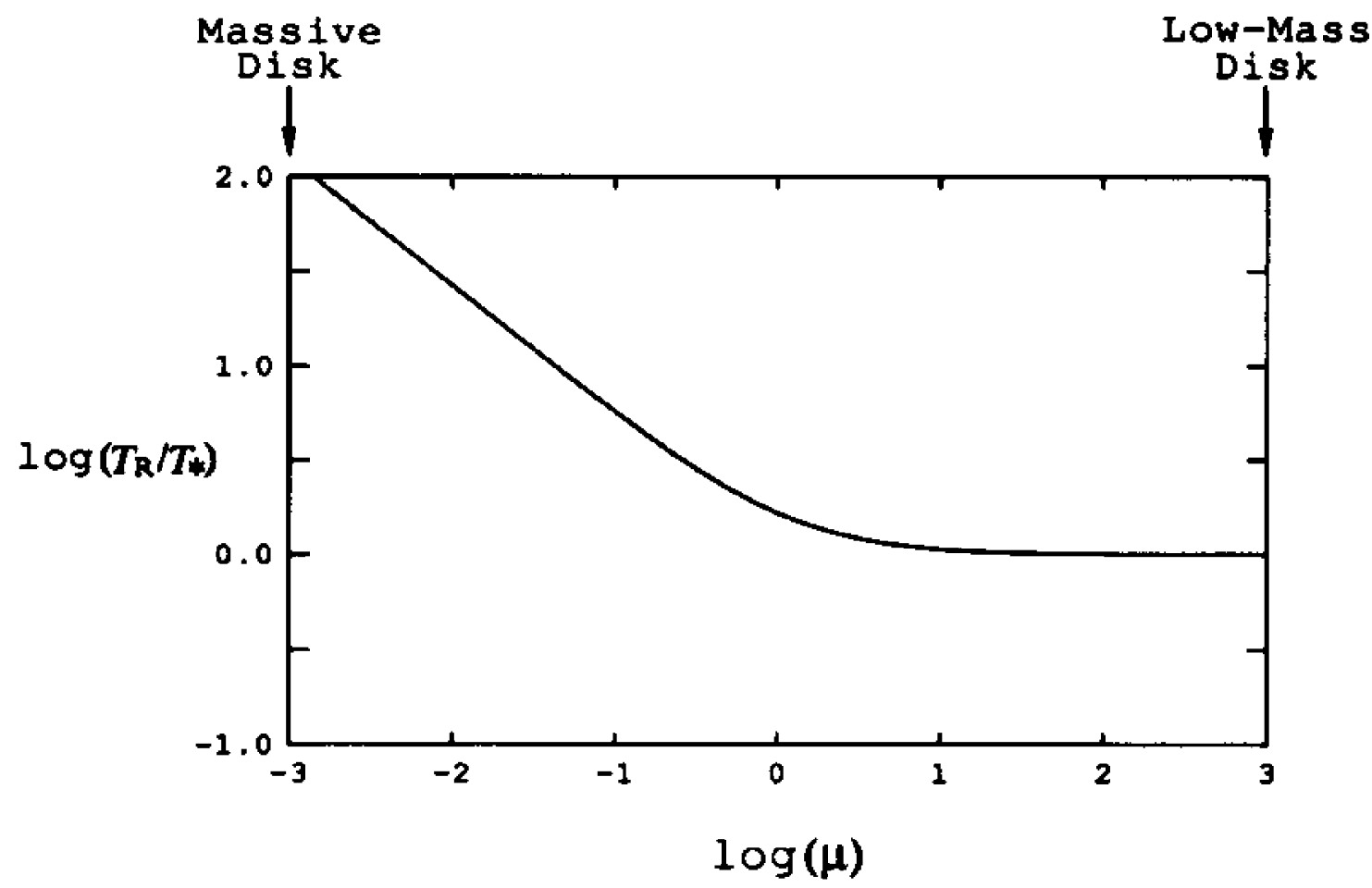

Figure 4.5: The curve in this diagram establishes a lower limit on the temperature ratio $\mathcal{T}_{R} / \mathcal{T}_{*}$ that is required in order for excretion to begin and, hence, tidal disruption of a central star to occur in a disk + central star system. For all systems in which the disk has a mass greater than or equal to the mass of the central star, tidal disruption will only occur if the mean temperature of the disk material is greater than the mean temperature of material in the central star.

with $\mu \lesssim 1$, the condition for tidal disruption of the central star becomes

$$
\left(\frac{c_{R}}{c_{\star}}\right)^{2} \gtrsim \alpha_{1}\left(1+\frac{1}{\mu}\right),
$$

or, equivalently,

$$
\frac{T_{R}}{T_{\star}} \gtrsim \alpha_{1}\left(1+\frac{1}{\mu}\right) .
$$

This condition has been illustrated in Fig. 4.5. Notice in particular that the ratio $\mathcal{T}_{R} / \mathcal{T}_{\star}$ is everywhere greater than unity. Hence, tidal disruption of the central star by a massive disk can only occur if the mean temperature of the disk material is greater than the mean temperature of the central star. 
In realistic astrophysical environments, it is extremely unlikely that a system composed of a disk plus central star will ever evolve to a state where the disk material is, in the mean, hotter than the central star. Material in the central star should normally be hotter than material in the disk because it has had to fall into a relatively deeper potential well. Furthermore, because its surface area is smaller, the central star must generally be less efficient than the disk at radiative cooling. Hence, although we have demonstrated that, in principle, a disk can tidally disrupt the central star that it surrounds, in practice the conditions necessary for this to happen seem unlikely to occur in nature.

When a gravitationally unstable, interstellar gas cloud collapses to form a new star, it is likely that a major fraction of the gas will fall onto a rotationally supported disk surrounding a dense, central core rather than falling directly onto the central object, itself (Cassen \& Moosman 1981; Terebey, Shu, \& Cassen 1984; Cassen, Shu, \& Tereby 1985; Adams, Lada, \& Shu 1987). In principle, the disk can become more massive than the newly forming, central protostellar object and exert a significant tidal influence on that central object. In this chapter, we have analyzed the conditions under which tidal disruption of the central object by a massive, axisymmetric disk is likely to occur and have concluded that, because the disk is generally expected to be cooler (in the mean) than the central object, tidal disruption is unlikely to occur in realistic systems. Hence, tidal disruption does not appear to be a viable mechaniom to limit the disk-to-central-object mass ratio of protostellar systems. It seems more likely that this mass ratio will be limited in practice by nonaxisymmetric instabilities that will develop in cool, massive disks such as the instabilities that are being thoroughly analyzed in accompanying chapters of this dissertation. 


\section{CHAPTER 5 \\ DISK INSTABILITIES RESULTING FROM INTERACTIONS \\ WITH THE CENTRAL POINT MASS}

\section{s.1 Introduction}

Now that one possible interaction between a disk with mass comparable to that of the central object it surrounds has been examined with emphasis on the central object, it becomes important to examine the stability of the disk. To enable dynamical interactions between the disk and central object in our simulations, the $\pi$-symmetry constraint must be removed and the central object must not be artificially constrained to lie on the axis of the computational grid at all times. Little has been done in the area in which we are concerned about here. There has been a recent proposal that such systems may be unstable to an $m=1$ instability and dynarnically generate tightly wound, one-armed spirals through disk interactions with a freely moving central object (Adams et al., 1989; Shu et al., 1990). Additionally, there has been a two-dimensional, nonlinear study of disk-central object systems with significant mass contained in each component, treating the central object as a fixed point source, but relaxing the $\pi$-8ymmetry constraint of Chapters 2 and 3 (Papaloizou and Savonije 1991). As the principal focus of this dissertation is the linear instability of disk systems, and this chapter concerns itself with the removal of the $\pi$-8ymmetry constraint, it is the work of Adams et al. that is the groundwork upon which the following analysis is built.

One very unique characteristic of systems with disk masses comparable to that of the central object is that the central object's position is not necessarily the same as that of the center of mass of the system. In such a case, the central object 
responds to the gravitational influence of the disk, whereas in other cases, the central object either dominates the system to such an extent that the gravitational influence of the disk is negligible, or else the central object itself is of little importance compared to the disk. The questions we seek to answer, then, are what mass ratios are unstable to a disk-central object interaction, and what form does the mode of instability take? To that end, $\$ 5.2$ examines, in a relatively crude manner, the motion of the central object due to the gravitational influence of the disk. Section 5.3 reviews the linear stability analysis of such interactions as first examined by Adams et al. (1989), while $\$ 5.4$ and $\$ 5.5$ discuss computational results performed in the same manner as the stability analysis presented in Chapter 2.

\subsection{Resonant Mass Ratio - Crude Calculation}

The mass ratios that are most unstable to a resonant interaction of the disk with the central object can be calculated rather easily in a crude fashion. For this calculation, we adopt - as in the axisymmetric Roche problem - a disk approximated by an infinitesimally thin ring and a central object represented by a point mass. As a further approximation, we first examine only radial motions within the system, disregarding any angular motions that may be introduced by restoring forces such as epicyclic motion. We can then write down a radial equation of motion for the central object,

$$
\ddot{r}=-\frac{\partial}{\partial r} \phi_{R}\left(r, r_{0}\right),
$$

where $r$ is the radial position of the central object measured from the center of mass of the axisymmetric ring, and $r_{0}$ is the radius of the ring. From equation (4.1b), the gravitational potential of the ring is, simply,

$$
\phi_{R}=-\frac{G M_{R}}{r_{0}} \frac{2}{\pi} K\left[(\alpha)^{2}\right],
$$


where we have defined $\alpha \equiv r / r_{0}$, as in Chapter 4 . Hence, the equation of motion of the central object can be rewritten in the form

$$
\ddot{\alpha}=\frac{G M_{R}}{r_{0}^{3}} \frac{2}{\pi}\left[\frac{E\left(\alpha^{2}\right)}{\alpha\left(1-\alpha^{2}\right)}-\frac{K\left(\alpha^{2}\right)}{\alpha}\right] .
$$

Assuming an exponential form for the time-variation of $\alpha$,

$$
\alpha=a e^{\mathrm{j} \omega t},
$$

we can solve for the dispersion relationship for the oscillatory frequency of the central object, $\omega$. We find

$$
\omega^{2}=-\frac{G M_{R}}{\alpha^{2} r_{0}^{3}} \frac{2}{\pi}\left[\frac{E\left(\alpha^{2}\right)}{1-\alpha^{2}}-K\left(\alpha^{2}\right)\right],
$$

and we note that for any $\alpha^{2}<1, \omega^{2}<0$. Hence, in the absence of any restoring forces, $\alpha$ grows exponentially. The point mass simply "rolls" off the top of the $\phi_{R}$ potential well, while the ring is displaced in the opposite direction, leaving the center of mass of the system in a fixed location.

Expression (5.5) allows us to estimate the growth rate for this simplified situation of purely radial motion in a straightforward manner. By assuming only small values of $\alpha$, we may use an expansion for the elliptic integrals as given in Arfken (1985),

$$
\begin{aligned}
E\left(\alpha^{2}\right) & =\frac{\pi}{2}\left[1-\left(\frac{1}{2}\right)^{2} \alpha^{2}-O\left(\alpha^{4}\right)\right], \\
K\left(\alpha^{2}\right) & =\frac{\pi}{2}\left[1+\left(\frac{1}{2}\right)^{2} \alpha^{2}+O\left(\alpha^{4}\right)\right] .
\end{aligned}
$$

Hence,

$$
\omega^{2} \approx-\frac{1}{2} G M_{R} \frac{1}{r_{0}^{3}}
$$

and, from eq. (2.10),

$$
y_{2}(1) \approx\left[\frac{G M_{R}}{\left(2 r_{0}^{3} \Omega_{0}^{2}\right)}\right]^{1 / 2}
$$


This behavior occurs for any mass ratio, as restoring forces have not yet been considered.

Since $\omega$ is the frequency with which the central object "oscillates" about the center of mass of the ring, it is appropriate to compare this frequency to the epicyclic motion of particles in the disk. In the case of a disk obeying a Keplerian rotation law, the epicyclic frequency, defined as in equation (2.16), is identical to the Keplerian orbital frequency $\Omega=\sqrt{G M_{\mathrm{c}}} \mathrm{r}^{-3 / 2}$. Thus, the epicyclic frequency of our thin hoop approximation to the disk is

$$
\kappa^{2}=\frac{G M_{c}}{r_{0}^{3}} .
$$

The ratio of $\omega^{2}$ to $\kappa^{2}$ is then given by

$$
\left|\frac{\omega^{2}}{\kappa^{2}}\right|=\frac{M_{R}}{M_{c}} \frac{2}{\pi \alpha^{2}}\left[\frac{E\left(\alpha^{2}\right)}{1-\alpha^{2}}-K\left(\alpha^{2}\right)\right] .
$$

Again, assuming small values of $\alpha$, substitution of expressions (5.6a) and (5.6b) into Equation 5.10 gives

$$
\left|\frac{\omega^{2}}{\kappa^{2}}\right| \approx \frac{M_{R}}{M_{c}} \frac{1}{\pi}\left[\frac{1+\frac{1}{2} \alpha^{2}}{1-\alpha^{2}}\right] .
$$

Thus, if a resonance exists between the central object's orbital frequency, and the epicyclic frequency of particles in the disk $(\omega=\kappa)$, the mass ratio of the system (given $\alpha=0$ as an initial condition) would be

$$
\frac{M_{R}}{M_{c}}=\pi
$$

Hence, we would expect the restoring force present in the ring to be of importance in accretion disk systems in which the mass ratio is near unity. Of course, if the mass ratio were much less than unity, $\left(M_{R} / M_{c}<<1\right)$, the disk would not have enough mass to influence the central object in a significant manner. Likewise, if the mass ratio were much greater than unity, $\left(M_{R} / M_{c}>>1\right)$, the reverse situation 
would exist. Having an insignificant mass, the central object should not influence the disk significantly. The analysis used here to arrive at this conclusion has been relatively simple and crude, revealing little about the growth rate and pattern speed of the resonant interaction or about the resulting structure that will develop in the disk. For such matters, more detailed calculations must be performed.

\subsection{Linear Theory Predictions}

The previous method of handling this problem gives only a very approximate idea of the kind of behavior one can expect. One way to handle this problem more accurately is to write out the relevant hydrodynamic equations, linearize them, and then solve the resulting eigenvalue problem. This has been done in some detail by others for the case of two-dimensional, near-Keplerian systems (see Adams et al, 1989; Shu et al., 1990). As a prelude to our computational survey of the stability of fully three-dimensional, thick disks, we review qualitatively the results of this linear treatment of the interactions of a thin, extended disk with a central object.

In the Adams et al. analytical treatment of accretion disk systerns where $M_{d} / M_{c} \sim 1$, the central object is allowed to respond to the gravitational influence of the disk; hence, the central object is not constrained to remain at the center of mass of the system. The resulting potential of the central object is then treated as the potential of an object positioned at the center of mass of the system, plus an indirect potential. It is the interaction of the disk with this indirect potential that results in the development of an instability within the disk. Through a series of linear computations, the central object is observed to spiral outward, away from the center of mass, due to the influence of the disk. In response, the disk is observed to develop an $m=1$ mode instability, reacting in such a way as to constrain the center of mass of the combined system to lie at its original position. The perturbation in 
the disk develops a tightly wound, trailing spiral character. Material accumulates on one side of the disk, and Adams et al. speculate that in the nonlinear regime, this accumulation might become an orbiting companion to the central object. The suggestion is made as well that as a protostellar disk continually gains material from an infalling gas cloud, the central object may spiral out to a sufficiently large position as to "dredge" material out of the inner regions of the disk. In this rather catastrophic manner, the disk could effectively reduce its mass. With these ideas in mind, we proceed to examine such systems computationally, modifying the hydrodynamic code to treat the central object as a dynamic object. This modified code is then used to identify marginally unstable systems, as was done in Chapter 3.

\subsection{Computational Considerations}

The hydrodynamic code used in Chapters 2 and 3 required modification to ease the constraint that the central object always remains fixed on the axis of the computational grid. In principle, this should be a straightforward process given that two methods of determining the position and velocity of the central object could be used. One method is to determine the position and velocity of the center of mass of the disk, then artificially move the central object in such a way that the position of the center of mass of the system lies fixed at the center of the computational grid. The difficulty with this method arises from the manner in which material reaching the outer edge of the computational grid is handled. In the current hydrocode, this material is removed from the system. As such, the center of mass of the disk may change artificially due to material exceeding the limits of the computational grid.

The second method of determining the position and velocity of the central object is through the direct time evolution of these quantities. The central object should move in response to the local gradient in the gravitational potential. Hence, 
from a finite-difference determination of this gradient, the instantaneous acceleration of the central object can be determined; the change in velocity and position over some time interval can then be determined from this acceleration. Difficulties with this method arise due to the singular nature of the central axis of a cylindrical coordinate system. Given a small radial displacement of the central object, $1 / r$ terms that result from the determination of the acceleration from the gravitational potential gradient yield artificially inflated values of the acceleration. At some distance away from the central axis (determined by trial and error to be less than one-half the radial extent of a single grid zone in the current hydrocode), the finite-difference technique begins to work extremely well.

The method used in the hydrocode for the evolution of models presented in this chapter is a combination of the two procedures detailed above. While the disk is not significantly distorted (i.e., while the amplitude of the instability is small), the position and velocity of the central object are determined from the center of mass of the disk. Once the radial position of the central object exceeds one-half of a grid zone, the changes to the position and velocity are followed explicitly through the determination of the force acting on the central object. This hybrid technique works well because, in practice, the amplitude of the disk distortion is correlated with the amplitude of the radial motion of the central object - as the disk distorts to a level where the first method becomes relatively uncertain, the central object has usually moved far enough from the axis that the second method becomes reliable.

As in Chapter 3, three different mass ratios are examined: $M_{d} / M_{c}=5.0,1.0$, and 0.2. Five, six, and four models are evolved for the three mass ratios 5.0, 1.0, and 0.2, respectively. Data describing all 15 initial models are given in Tables 5.1, 5.3, and 5.5. Measured $y_{1}(1)$ and $y_{2}(1)$ parameters for all models have been tabulated 
in Tables 5.2, 5.4, and 5.6, and displayed in Figures 5.1, 5.7, 5.14, and (the overall summary) 5.19 .

\subsubsection{Systems with $M_{d} / M_{c}=5.0$}

For the models with a mass ratio of 5.0 , an $m=1$ mode was found to be unstable. Examining only the two parameters $y_{1}(1)$ and $y_{2}(1)$ plotted as a function of $T /|W|$, as in Figure 5.1, one learns little more than what was discovered from the models in which we enforced $\pi$-8ymmetry (for comparison, see Figure 3.1 ). As before, the growth rates decrease smoothly from the highest $T /|W|$ model to the lowest. One difference lies in the derived value of the pattern speed parameter, which has a value approximately half that obtained from the high mass, $\pi$-symmetry models, and varies in value over the range of $T /|W|$ examined much more so than in the $\pi$-symmetry models. For the $m=1$ mode, the marginally unstable disk model is found to be somewhat "hotter" than it was for the $m=2$ mode - from a simple examination of the trend displayed in Figure 5.1, we estimate that the critically unstable model lies not far below model $\# 1$, at a value of $T /|W| \approx 0.1$. The pattern speed derived from $y_{1}(1)$ has a value $\Omega_{p} / \Omega_{0} \approx 0.3$, which is a factor of 1.7 smaller than what we found for the $m=2$ mode (see Table 3.2). Quantitative differences of this sort can arise naturally within the context of a single type of physical instability as one shifts the focus from the $m=2$ mode to the (longer wavelength) $m=1$ mode (cf. Kojima 1986; Christodoulou and Narayan 1992; and our earlier discussions in $\$ 2.6$ and $\$ 3.2 .3)$.

When one examines the radial structure of the $m=1$ eigenfunction, however, it becomes apparent that this instability is different in nature from the $m=2$ mode instability. The $\phi_{1}-r$ plots of Figures 5.2b-5.4b (models \#1-3) all display a trailing spiral character that is unlike any of our previously discussed models. This 
spiral mode is, however, very similar to the one predicted by Adams, Ruden, and Shu (1989) and discussed in \$5.3. The $m=1$ eigenfunction presents itself as a very pronounced spiral, clearly covering approximately $2 \pi$ of arc between the inner and outer edges of our disk. One effect of this developing mode is that, during the evolution, the center of mass of the disk steadily shifts away from the center of mass of the system. Furthermore, the spiral distortion effectively produces a single high density "blob" located radially near $r_{0}$ which orbits about the center of mass of the system along with (but oppositely positioned from) the central object. As the $m=1$ spiral distortion amplifies in the disk, the central object moves farther from the center of mass of the system along a tightly wound spiral trajectory. The upper panel in Figure 5.2c shows the path in the equatorial plane that the central object follows over the course of the evolution. The lower panel displays the logarithm of the displacement from the center of mass as a function of time. Note that if $\ln \left(r / r_{0}\right)$ is treated as $\ln D_{1}$ for the derivation of a growth rate parameter, the value of $y_{2}(1)$ found is identical to that derived from $\ln D_{1}$ itself. Hence, we have an alternative method of measuring the rate of growth of this $m=1$, global instability; one which relies on data that clearly exhibits less noise than the Fourier transform derived data.

There is considerable interest in the nonlinear outcome of these particular models, particularly with regard to the binary formation scenario proposed by Adams et al. and described in §5.3. The evolution of these models has, unfortunately, not been followed into the nonlinear regime due to computational difficulties that arise when the central object impacts the inner edge of the disk. When this occurs, the central object, with its singular $1 / r$ point-mass potential, interacts in a violent manner with the material in the gaseous disk. In the future, we may soften the potential in some manner in the immediate vicinity of the central object in an 
attempt to prevent such a violent impact from occurring and allow the evolution of these models to continue.

Models \# 4 and 5 show a different behavior altogether from models \#1-3. The $D_{1}-t$ plots (Figures 5.5 and 5.6 ) show clean "linear" behavior for both models, and the $\phi_{1}-t$ plots allow the easy determination of a pattern speed. The $y_{1}(1)$ and $y_{2}$ (1) parameters are not significantly different from those obtained for the spiral mode in models \#1-3. When we examine the $\phi_{1}-r$ plots, however, it is clear that the character of the instability has changed. The broad spiral observed in models \#1-3 is gone; it has been replaced by a leading phase shift of $\sim \pi$ radians in model \#4, and a leading spiral in model \#5. Given an understanding of the phase shift of $\pi / 2(\S 2.5)$, it is almost a trivial matter to picture what a phase shift of $\pi$ indicates. Instead of distorting the torus into an elliptical shape, if the torus were to be shifted as a whole a small amount away from the center of mass of the system, each circular annulus at $r<r_{0}$ would exhibit an azimuthal density maximum at a phase orientation of the nearest portion of the inner edge of the disk. As one crosses $r_{0}$ and examines annuli with $r>r_{0}$, this phase orientation shifts by $\pi$ as the azimuthal density maximum is followed. Hence, $\phi_{1}-r$ plots which display a phase shift of $\pi$ radians signal evolutions in which the disk is simply shifting off axis, or "sliding" off the center of mass of the system. A pattern speed of precisely zero frequency (and, correspondingly, a value of $y_{1}(1)=-1$ exactly - gee eq. [2.10]) would occur if the model were not rotating. This may explain the behavior observed in model \#4. The behavior observed in model \#5, however, is not understood at this time.

We should point out that, although the $m=1$ mode is observed to be the most unstable mode in models \#1 - \#5, the $m=2$ mode can be observed to grow in the colder models. Model \#5, for example, is determined to have a value 
of $y_{2}(2)=0.40$ (measured directly from Figure 5.6a). This is entirely consistent with the growth rates that were measured for the $m=2$ mode in the mass ratio 5.0 models of Chapter 3 (see Table 3.2). In addition, the measured pattern speed parameter $y_{1}(2)=-0.90$ agrees quite well with the data of Chapter 3. 
Table 5.1

Initial Model Data for $M_{d} / M_{c}=5.0$

\begin{tabular}{cccc}
\hline \hline Model \# & $r_{-} / r_{+}$ & $r_{0} / r_{+}$ & $T /|W|$ \\
1 & 0.051 & 0.127 & 0.102 \\
2 & 0.066 & 0.175 & 0.120 \\
3 & 0.111 & 0.287 & 0.166 \\
4 & 0.185 & 0.416 & 0.220 \\
5 & 0.296 & 0.565 & 0.279 \\
\hline
\end{tabular}


Table $\mathbf{6 . 2}$

m=1 Eigenmode Character, $M_{d} / M_{c}=5.0$

\begin{tabular}{cccccc}
\hline \hline Model & $y_{1}$ & $y_{2}$ & $\Omega_{p} / \Omega_{0}$ & $r_{c r} / r_{0}$ & $r_{c r} / r_{+}$ \\
1 & -0.70 & 0.077 & 0.30 & 1.84 & 0.23 \\
2 & -0.53 & 0.382 & 0.47 & 1.47 & 0.26 \\
3 & -0.49 & 0.710 & 0.51 & 1.40 & 0.40 \\
4 & -0.55 & 0.887 & 0.45 & 1.49 & 0.62 \\
5 & -0.73 & 0.888 & 0.27 & 1.93 & 1.09 \\
\hline
\end{tabular}



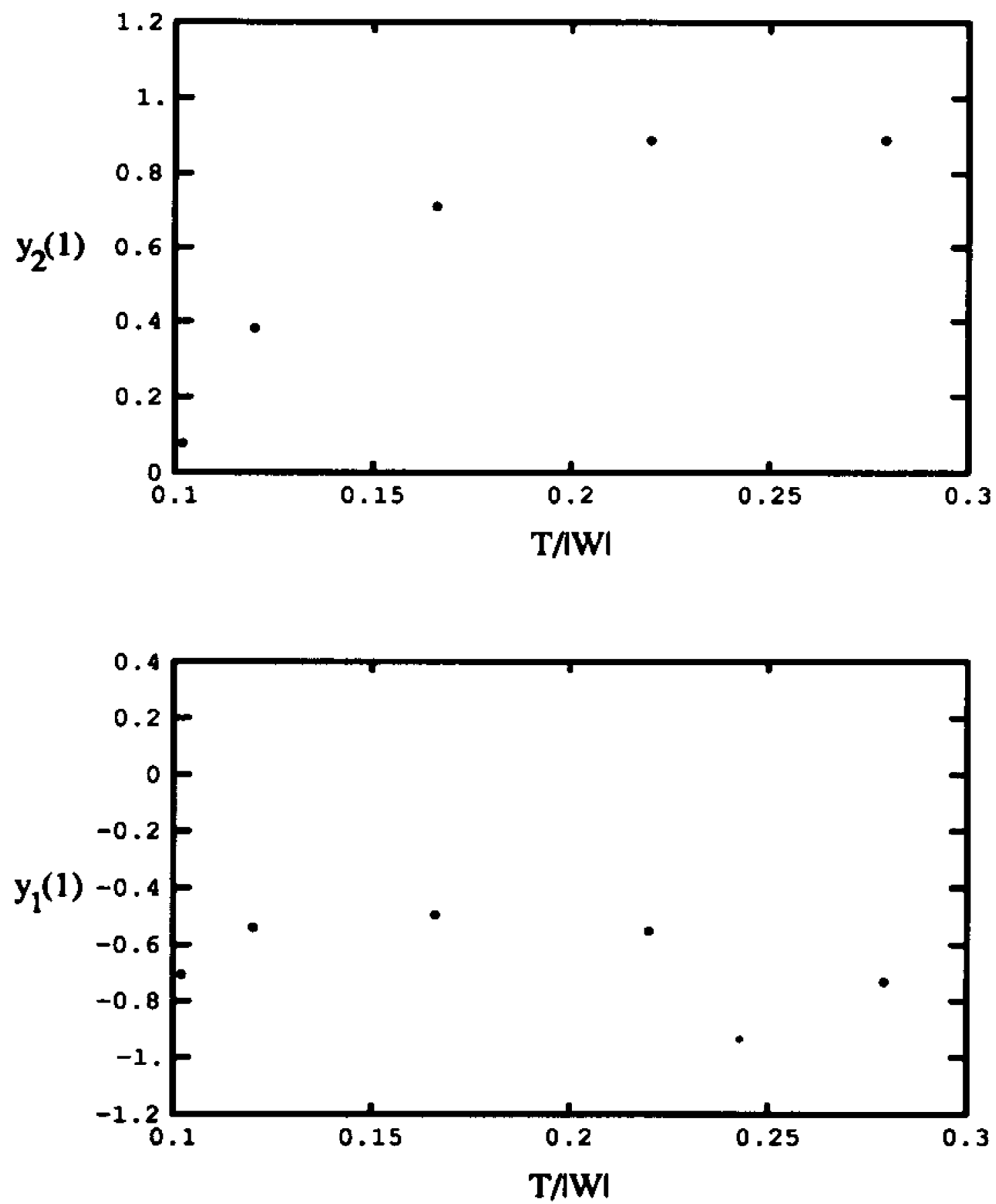

Figure 5.1: $y_{2}(1)$ and $y_{1}(1)$ as functions of $T /|W|$ for $M_{d} / M_{c}=5.0$ models. 

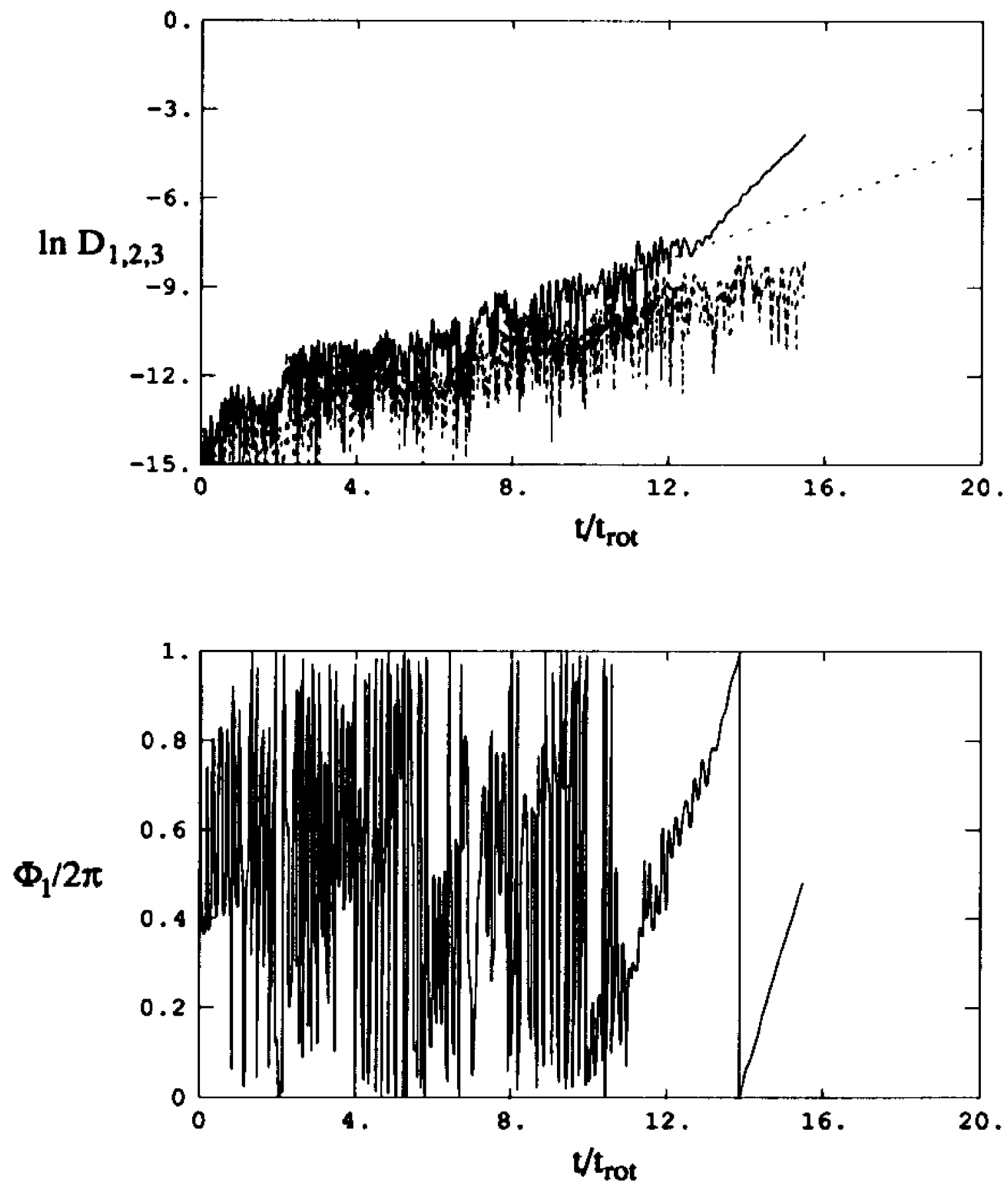

Figure 5.2a: The $m=1, m=2$, and $m=3$ Fourier amplitude behavior, and the $m=1$ phase behavior as a function of time, illustrated as in Figure 2.3a for $M_{d} / M_{c}=5.0$, model \#1. 

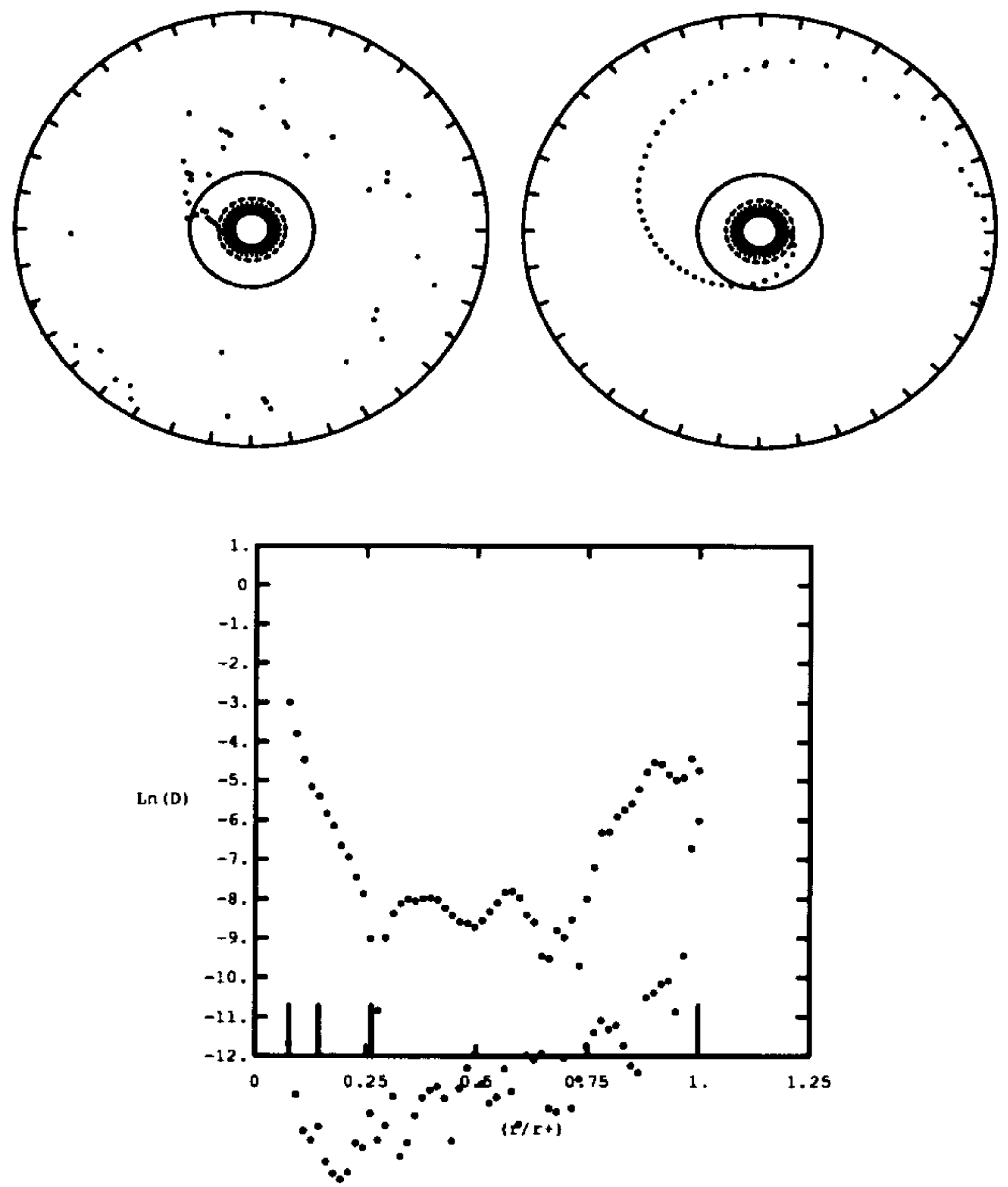

Figure 5.2b: The radial eigenfunction $\left(\phi_{1}-r\right)$ and amplitude as a function of radius $\left(D_{1}-r\right)$ of the $m=1$ Fourier mode illustrated as in Figure $2.3 \mathrm{~b}$ for $M_{d} / M_{c}=5.0$, model \#1. Note the development of a broad onearmed spiral in the upper panels. 

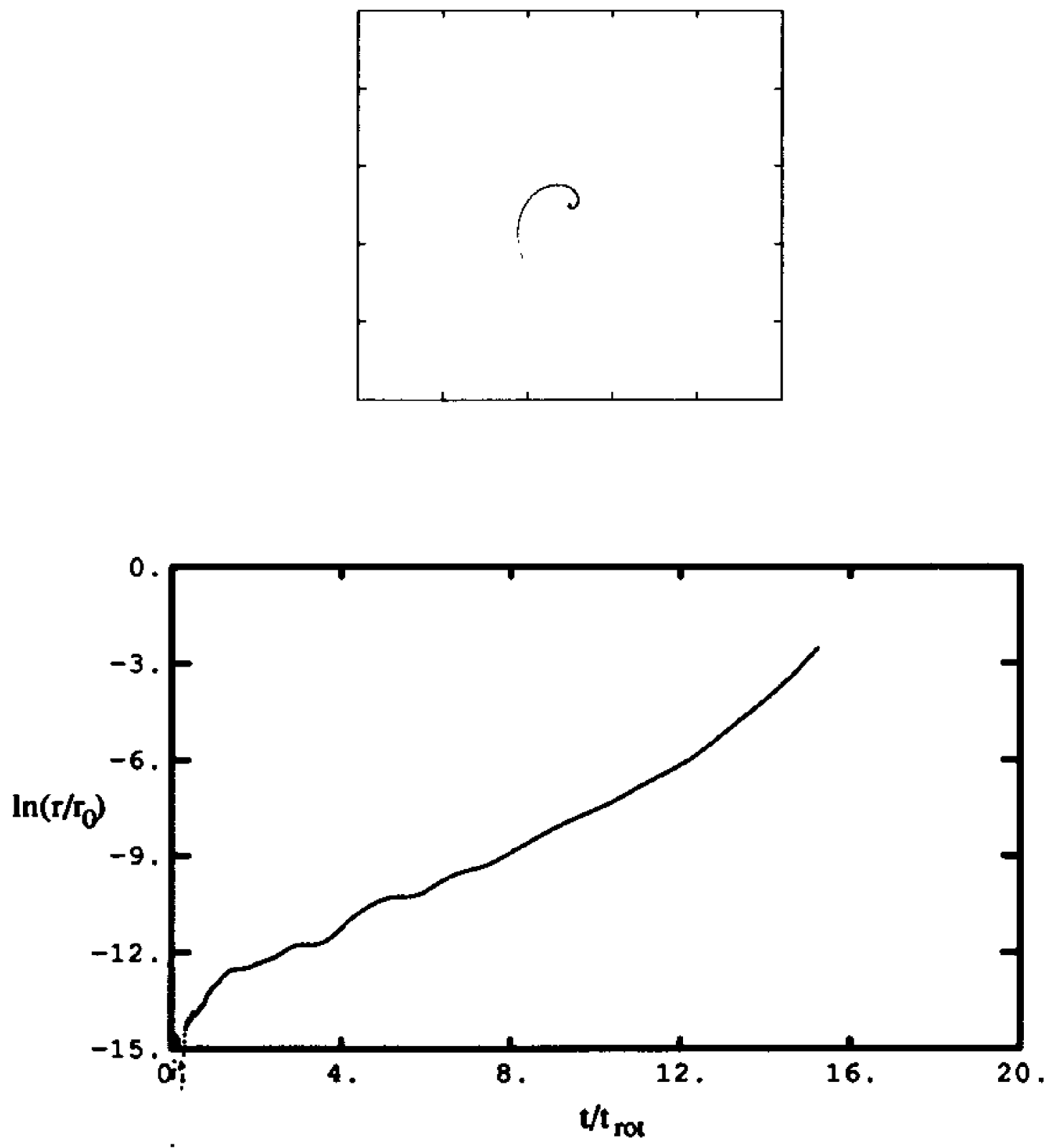

Figure 5.2c: The positional behavior of the central object over the course of the evolution of model \#1 (upper panel), and the logarithm of the radial position of the central object as a function of time (lower panel). Each tick-mark in the upper panel represents $\sim 1 / 10$ of a grid zone (in this particular case, the maximum radial position is $r / r_{0}=0.0589$ ). 



Figure 8.3a: The $m=1, m=2$, and $m=3$ Fourier amplitude behavior, and the $m=1$ phase behavior as a function of time, illustrated as in Figure $2.3 \mathrm{a}$ for $M_{d} / M_{c}=5.0$, model \#2. 

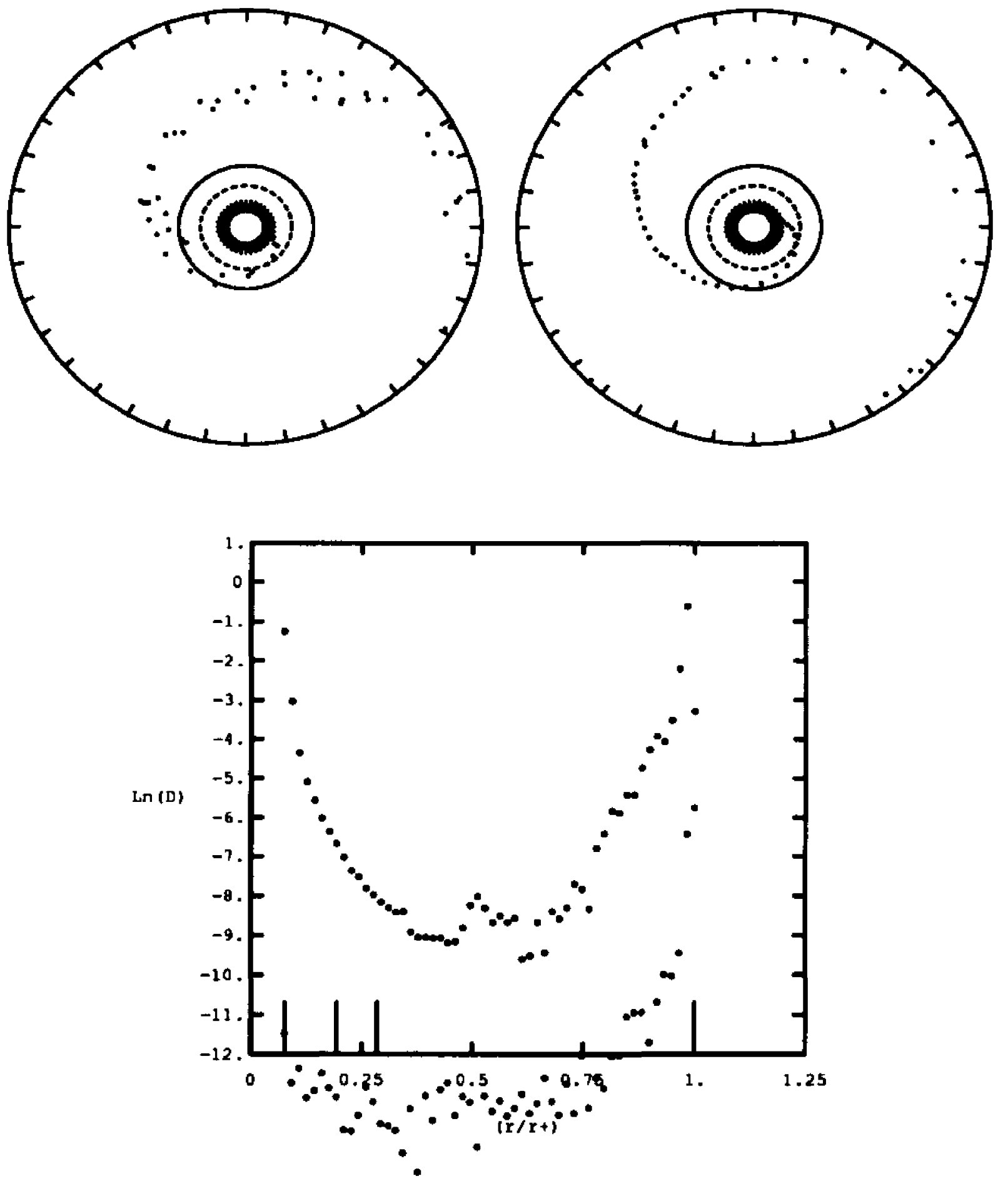

Figure 5.3b: The radial eigenfunction $\left(\phi_{1}-r\right)$ and amplitude as a function of radius $\left(D_{1}-r\right)$ of the $m=1$ Fourier mode illustrated as in Figure 2.3b for $M_{d} / M_{c}=5.0$, model \#2. 

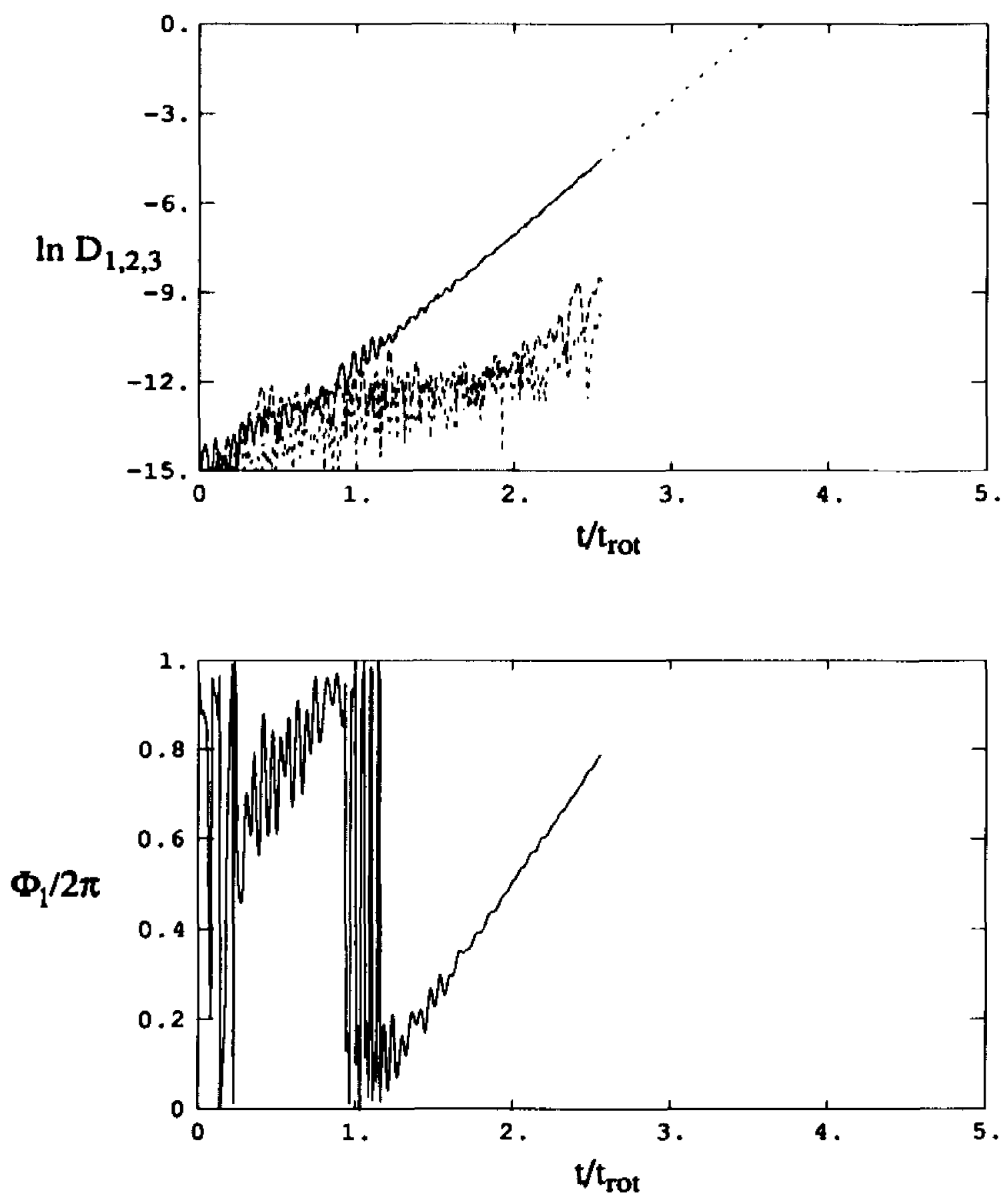

Figure 5.4a: The $m=1, m=2$, and $m=3$ Fourier amplitude behavior, and the $m=1$ phase behavior as a function of time, illustrated as in Figure 2.3a for $M_{d} / M_{c}=5.0$, model \#3. 

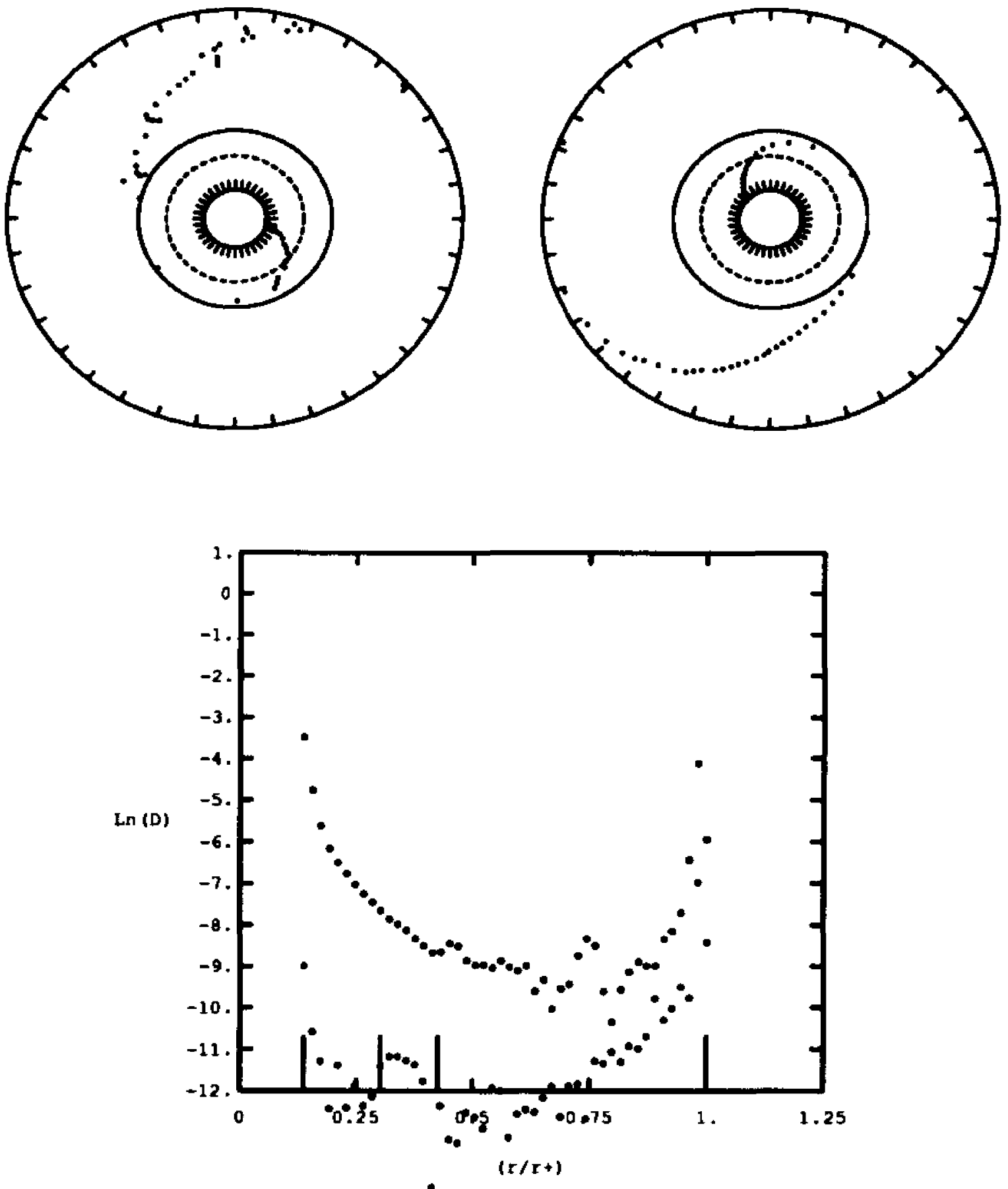

Figure 5.4b: The radial eigenfunction $\left(\phi_{1}-r\right)$ and amplitude as a function of radius $\left(D_{1}-r\right)$ of the $m=1$ Fourier mode illustrated as in Figure $2.3 \mathrm{~b}$ for $M_{d} / M_{\mathrm{c}}=5.0$, model \#3. 

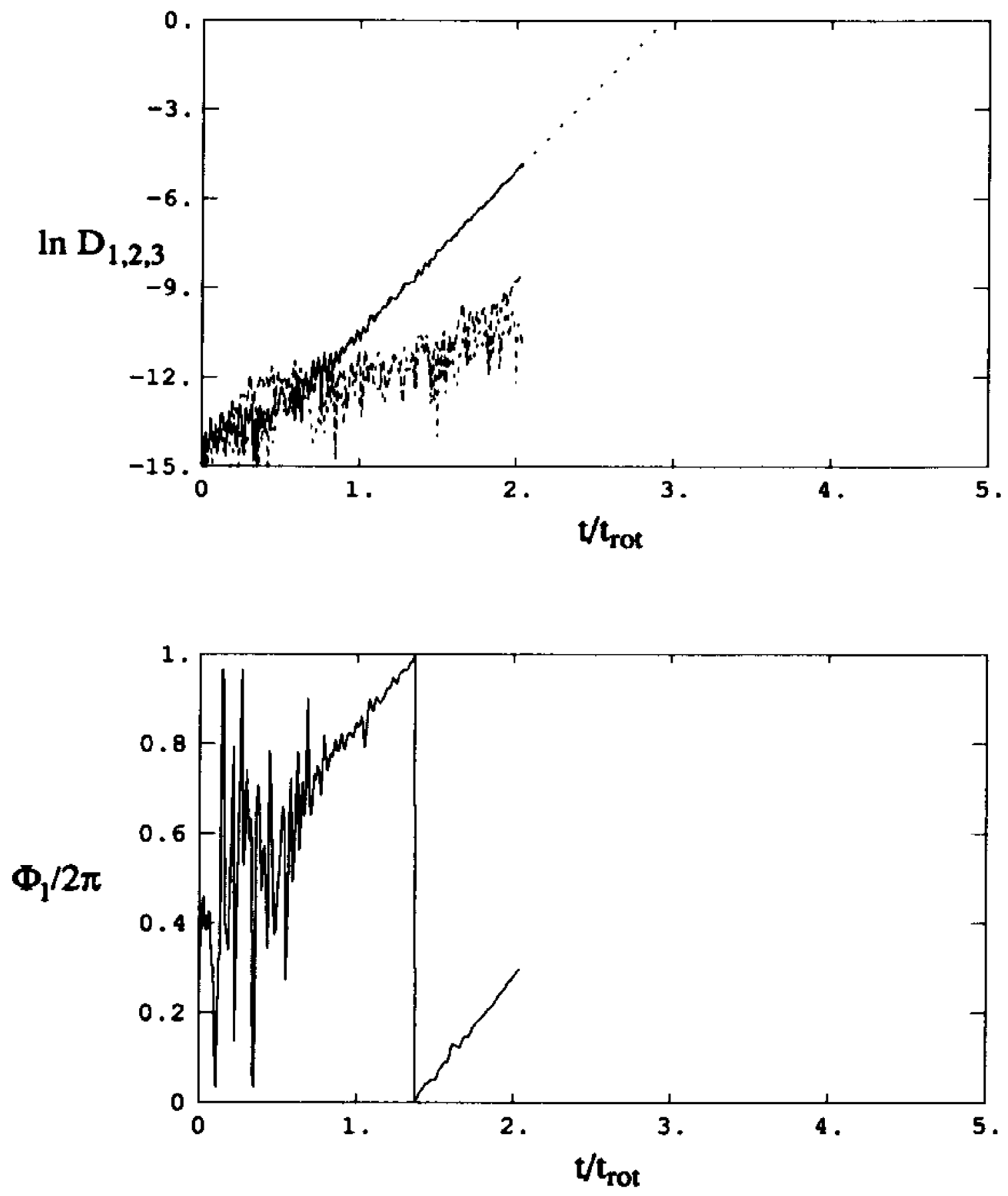

Figure 5.Sa: The $m=1, m=2$, and $m=3$ Fourier amplitude behavior, and the $m=1$ phase behavior as a function of time, illustrated as in Figure 2.3a for $M_{d} / M_{c}=5.0$, model \#4. 

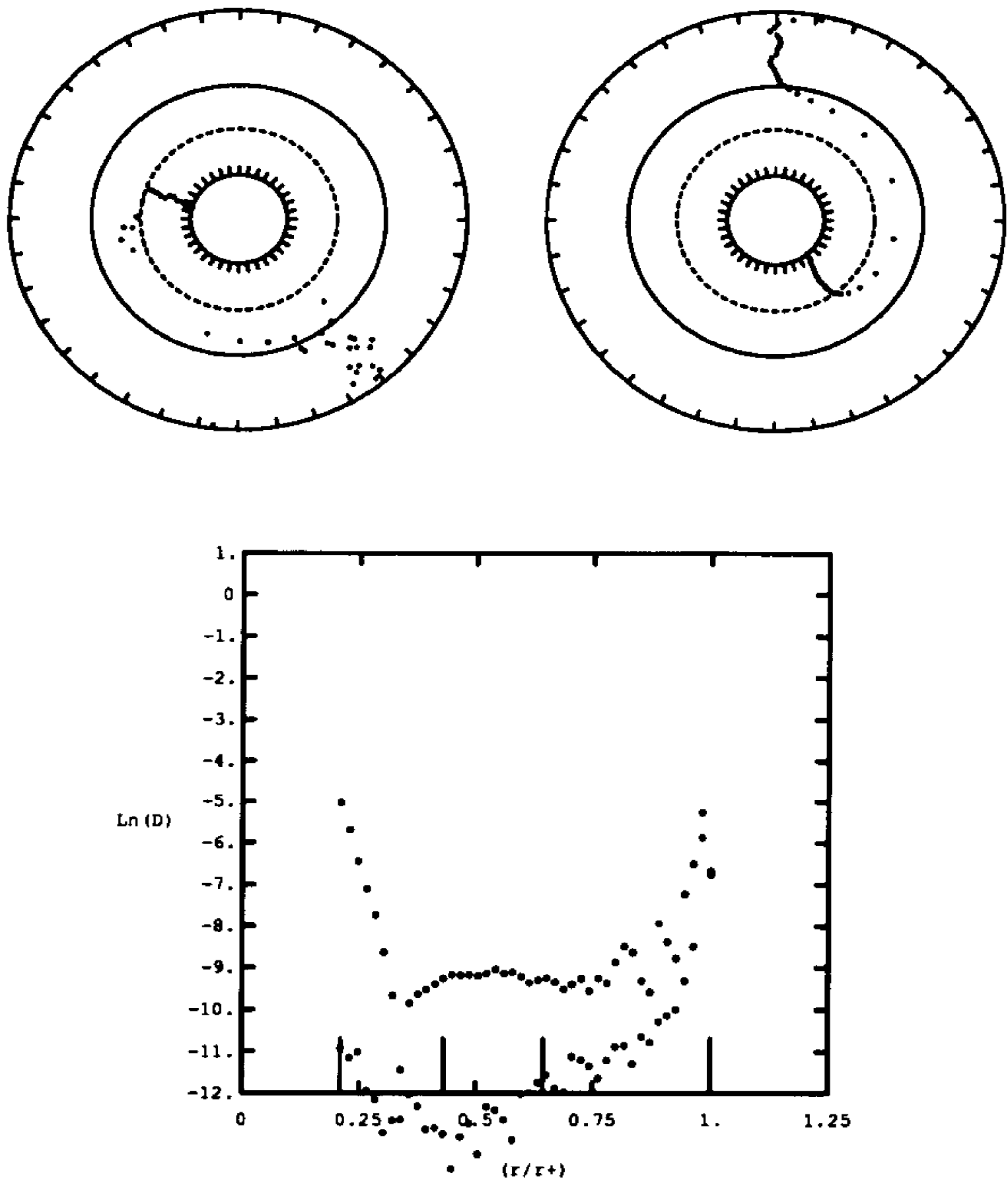

Figure 5.5b: The radial eigenfunction $\left(\phi_{1}-r\right)$ and amplitude as a function of radius $\left(D_{1}-r\right)$ of the $m=1$ Fourier mode illustrated as in Figure $2.3 \mathrm{~b}$ for $M_{d} / M_{\mathrm{c}}=5.0$, model \#4. 

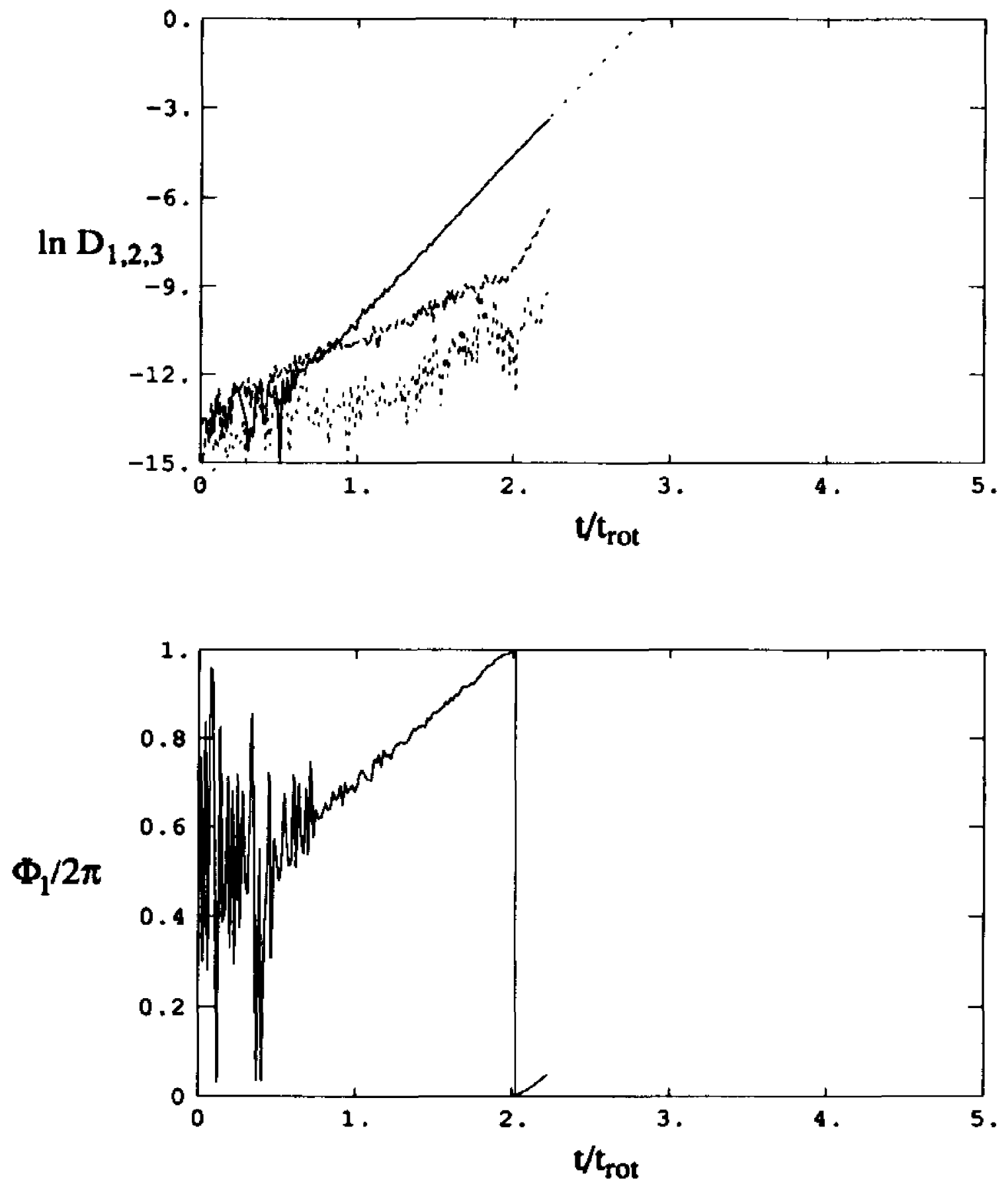

Figure 5.6a: The $m=1, m=2$, and $m=3$ Fourier amplitude behavior, and the $m=1$ phase behavior as a function of time, illustrated as in Figure 2.3a for $M_{d} / M_{c}=5.0$, model \#5. 

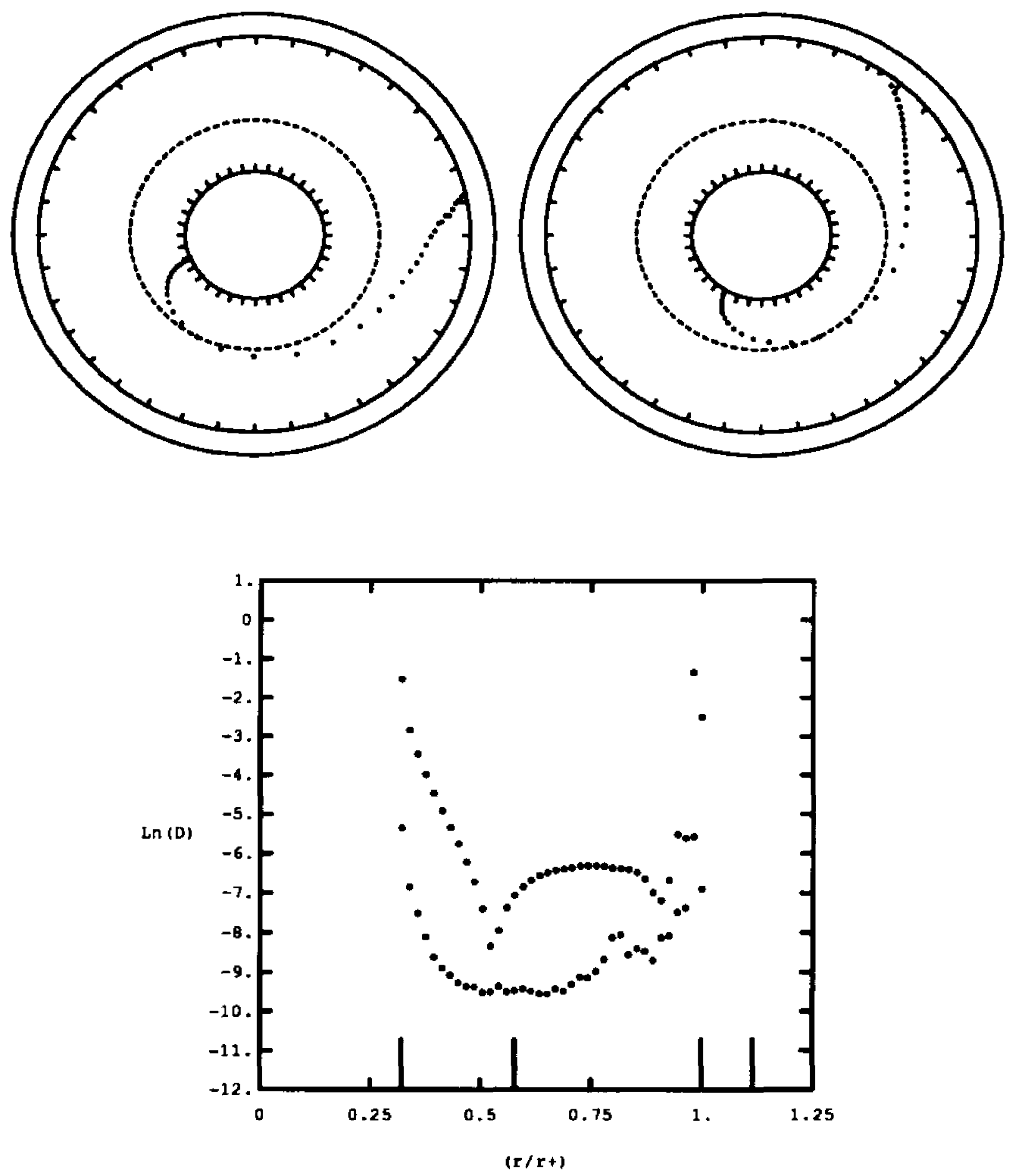

Figure 5.6b: The radial eigenfunction $\left(\phi_{1}-r\right)$ and amplitude as a function of radius $\left(D_{1}-r\right)$ of the $m=1$ Fourier mode illustrated as in Figure 2.3b for $M_{d} / M_{c}=5.0$, model \#5. 


\subsubsection{Systems with $M_{d} / M_{c}=1.0$}

Examination of the $m=1$ growth rate parameters for the models with a mass ratio of unity (see Figure 5.7 and Table 5.4 ) reveals the marginally unstable model to be in the vicinity of model $\# 1$, with $T /|W|=0.212$. Note, however, that as Figure 5.8a illustrates, model \# 1 is not purely "linear" in its growth. There is a period in the evolution of the model where the time variation of the Fourier mode amplitude becomes rather complicated in structure. For example, near $t / t_{\text {rot }} \approx 7$, the $m=2$ mode grows suddenly to become comparable in amplitude to the $m=1$ mode. We do not understand this behavior. Toward the end of the evolution, however, the $m=1$ mode clearly dominates. In this model, then, the value derived for $y_{2}(1)$ is sensitive to the time interval over which the slope is measured in the $D_{1}-t$ plot. The value given in Table $5.4-y_{2}(1)=0.027$ - has been measured over the latter half of the evolution and is shown in parentheses in order to indicate the uncertainty in its value.

As the top panels of Figs. $5.8 \mathrm{~b}$ and $5.9 \mathrm{~b}$ indicate, the $m=1$ mode that develops in models \#1 and \#2 displays the trailing spiral character that was observed in the majority of models with $M_{d} / M_{c}=5.0$. There is no significant difference in the measured pattern speeds of the two mass ratios (with the exception of model \#1 of $M_{d} / M_{c}=5.0$ ). The behavior of the eigenfunctions are identical. Hence, we conclude that the instability exhibited in models \#1 and \#2 is the same as that exhibited by models \#1-3 of the higher mass ratio.

Models \#3 - 6 display a different behavior from that of models \#1 and \#2. The spiral mode observed in models \#1 and \#2 is gone, replaced by a possible attempt at a spiral but with a phase shift occurring between the pressure maximum and the corotation radius. If the phase shift is measured with the inner edge of 
the disk used as one reference point and the corotation radius used as the other, then the phase shift is found to be nearly $\pi / 2$ radians in all four models. Note, however, that this $\pi / 2$ phase shift is in the opposite direction from the phase shift which was displayed by the $\pi$-symmetry models studied in Chapters 2 and 3. From a geometric perspective, we do not understand why a $\pi / 2$ phase shift should naturally be associated with an $m=1$ disk distortion, though we can tentatively identify the instability which exhibits such a behavior as the Papaloizou-Pringle P-mode instability (Papaloizou \& Pringle 1984; see also Appendix D). Note in addition that as one progresses from model \#3 to \#6, the spiral character outside of $r_{c r}$ decreases in extent, developing a somewhat leading character in model \#6. This behavior may be related to models \#4 and \#5 for $M_{d} / M_{c}=5.0$. However, $y_{1}(1) \approx-1 / 3$ for all 4 models with $M_{d} / M_{c}=1.0$, whereas $y_{1}(1)<-1 / 2$ for the corresponding models with $M_{d} / M_{c}=5.0$. 
Table 5.3

Initial Model Data for $M_{d} / M_{c}=1.0$

\begin{tabular}{cccc}
\hline \hline Model \# & $r_{-} / r_{+}$ & $r_{0} / r_{+}$ & $T /|W|$ \\
1 & 0.055 & 0.157 & 0.212 \\
2 & 0.092 & 0.231 & 0.251 \\
3 & 0.185 & 0.398 & 0.316 \\
4 & 0.222 & 0.453 & 0.334 \\
5 & 0.259 & 0.490 & 0.349 \\
6 & 0.296 & 0.546 & 0.362 \\
\hline
\end{tabular}


Table 5.4

m=1 Eigenmode Character, $M_{d} / M_{c}=1.0$

\begin{tabular}{cccccc}
\hline \hline Model & $y_{1}$ & $y_{2}$ & $\Omega_{p} / \Omega_{0}$ & $r_{c r} / r_{0}$ & $r_{c r} / r_{+}$ \\
1 & -0.46 & $(0.027)$ & 0.53 & 1.36 & 0.21 \\
2 & -0.50 & 0.179 & 0.49 & 1.42 & 0.32 \\
3 & -0.37 & 0.462 & 0.62 & 1.26 & 0.50 \\
4 & -0.34 & 0.492 & 0.65 & 1.23 & 0.56 \\
5 & -0.34 & 0.482 & 0.65 & 1.24 & 0.60 \\
6 & -0.30 & 0.518 & 0.69 & 1.19 & 0.65 \\
\hline
\end{tabular}



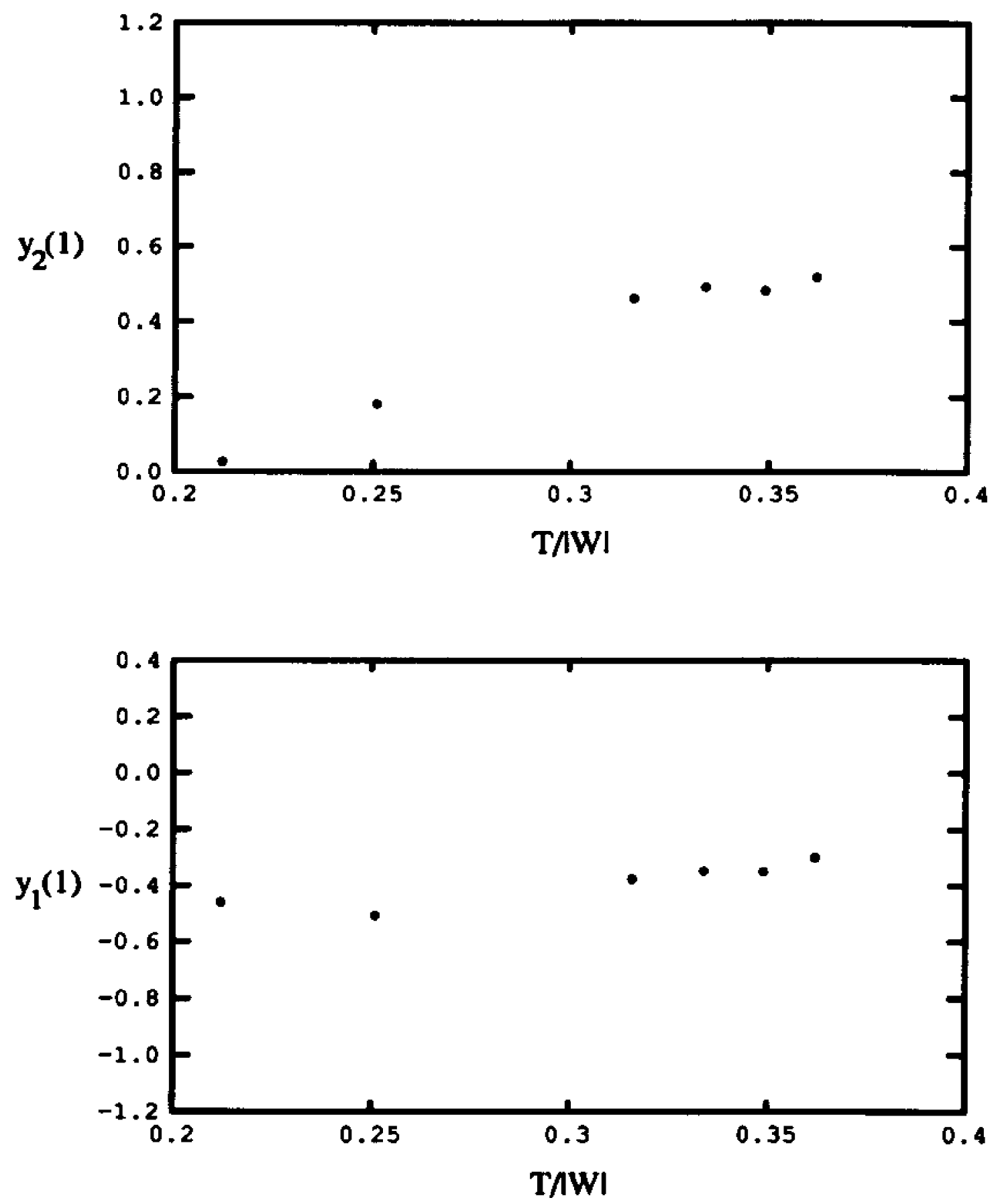

Figure 5.7: $y_{2}(1)$ and $y_{1}(1)$ as functions of $T /|W|$ for $M_{d} / M_{c}=1.0$ models. 

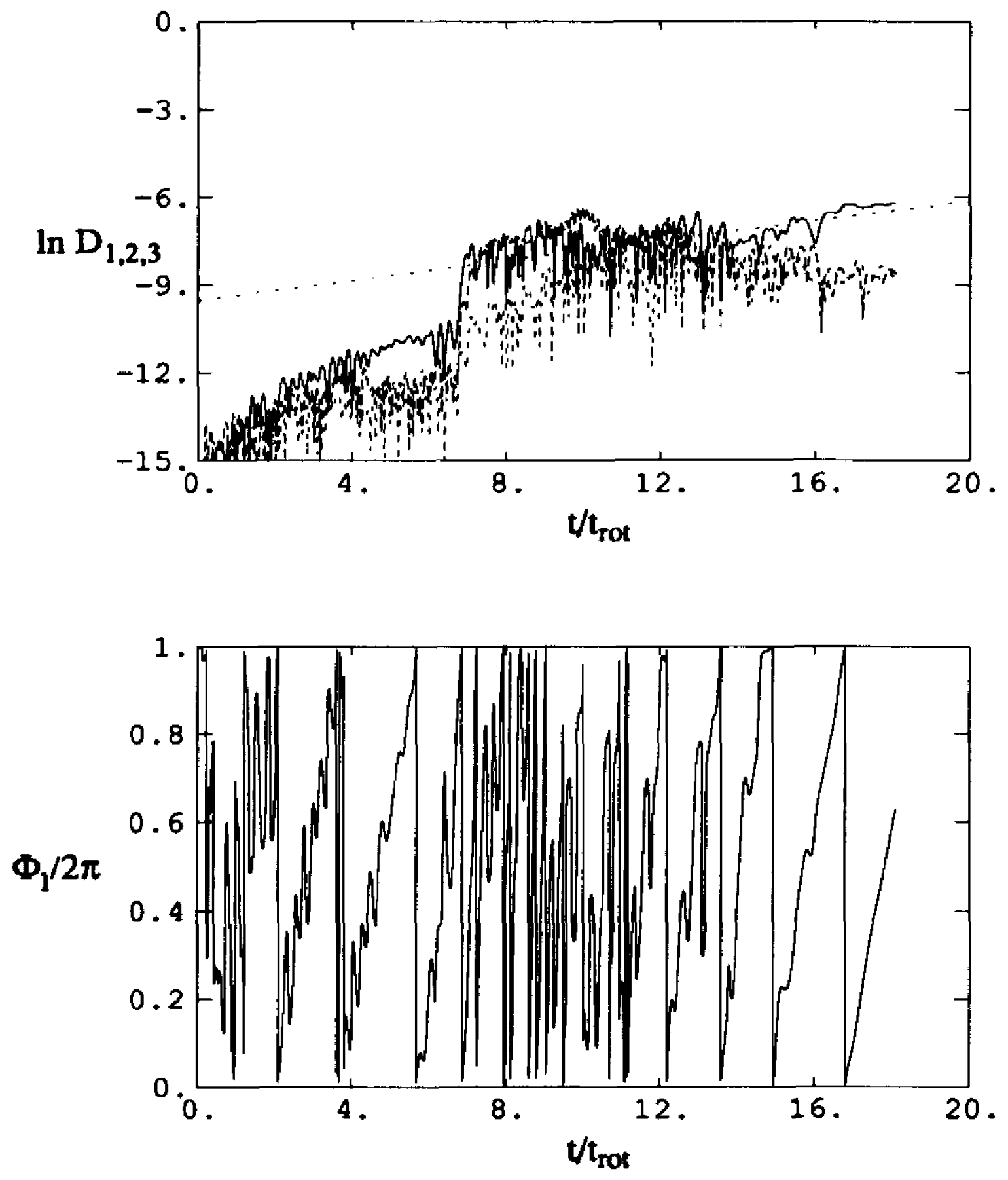

Figure 6.8a: The $m=1, m=2$, and $m=3$ Fourier amplitude behavior, and the $m=1$ phase behavior as a function of time, illustrated as in Figure 2.3a for $M_{d} / M_{c}=1.0$, model \#1. 

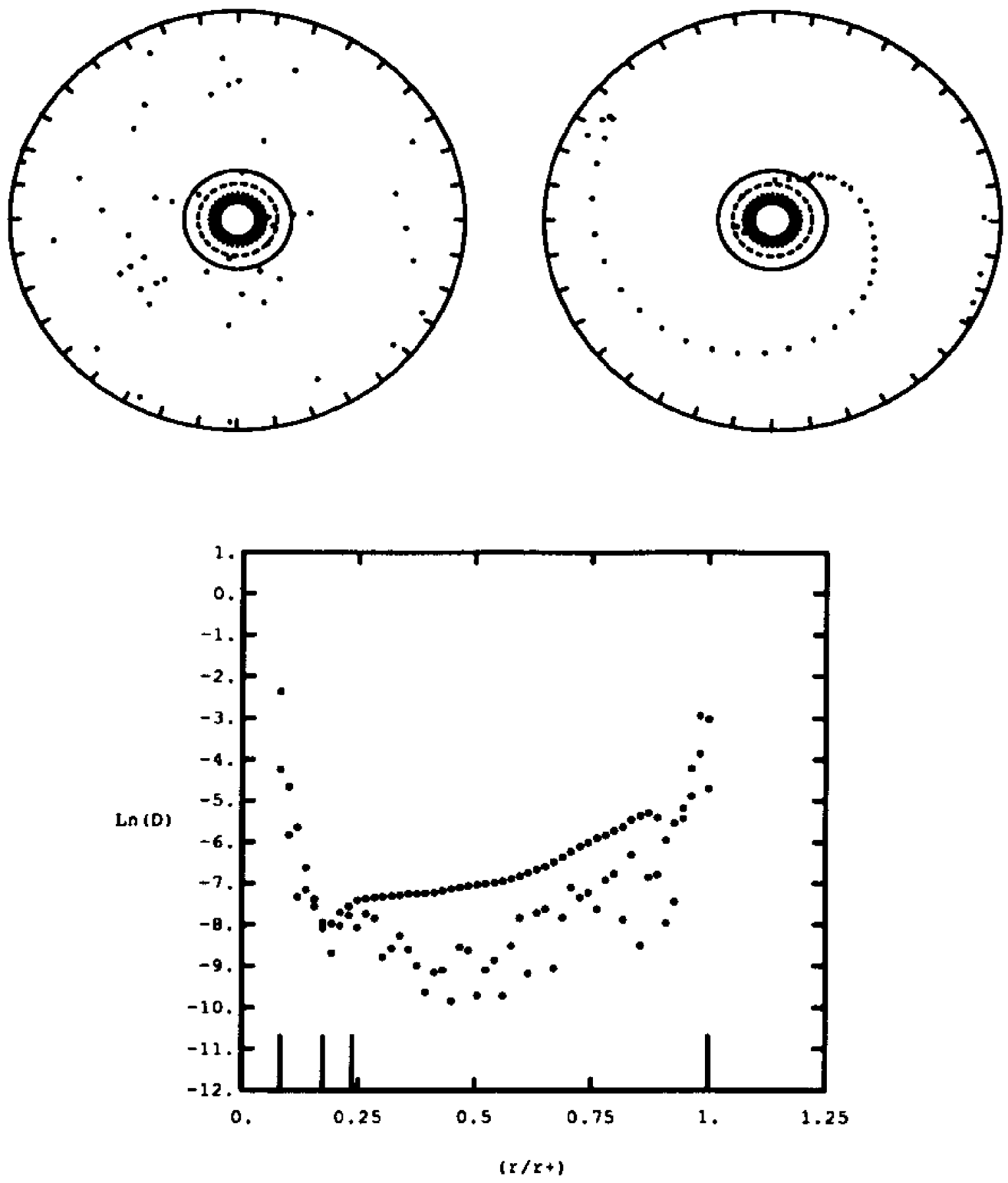

Figure 8.8b: The radial eigenfunction $\left(\phi_{1}-r\right)$ and amplitude as a function of radius $\left(D_{1}-r\right)$ of the $m=1$ Fourier mode illustrated as in Figure 2.3b for $M_{d} / M_{c}=1.0$, model \#1. 

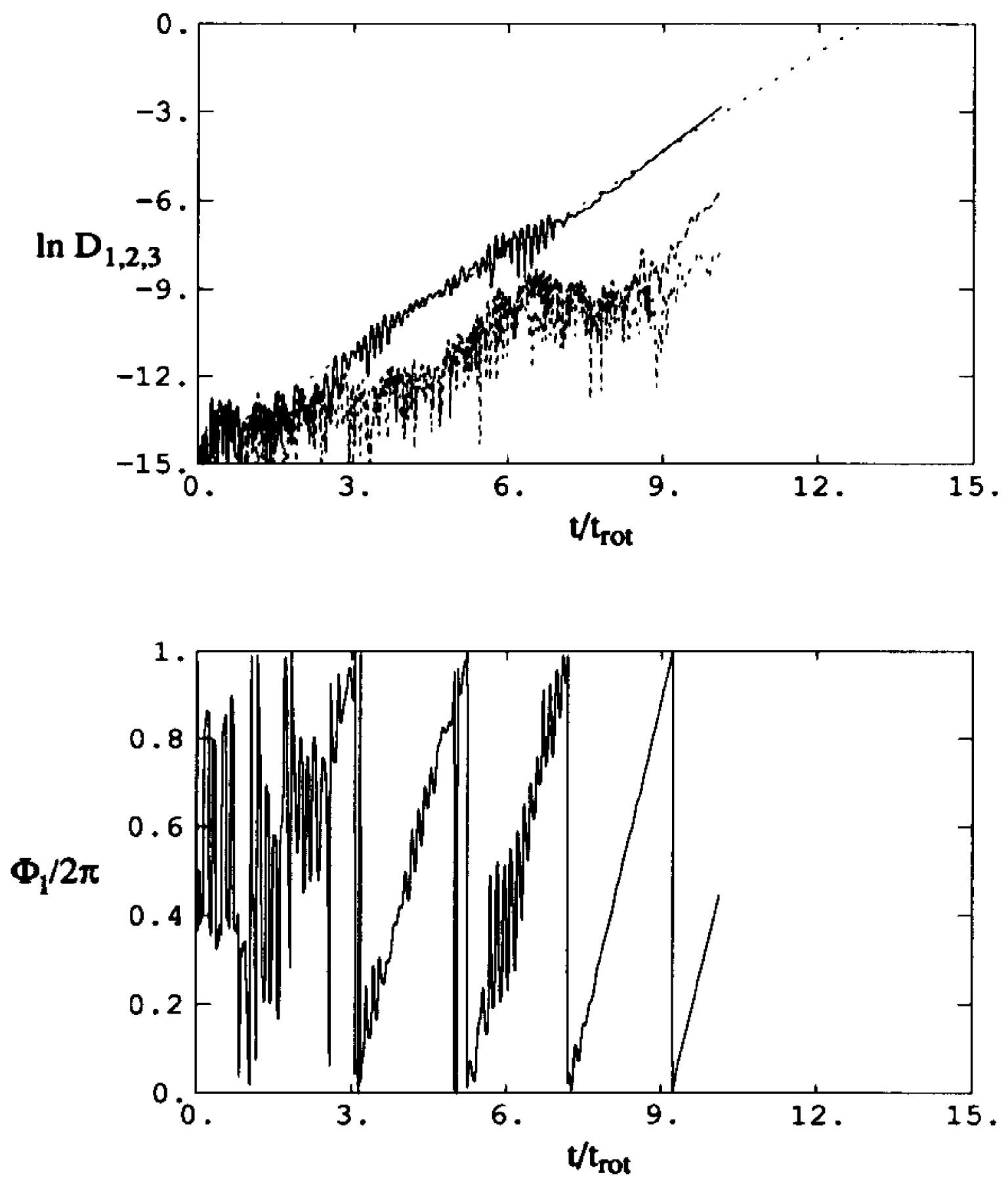

Figure 5.8a: The $m=1, m=2$, and $m=3$ Fourier amplitude behavior, and the $m=1$ phase behavior as a function of time, illustrated as in Figure 2.3a for $M_{d} / M_{c}=1.0$, model \#2. 

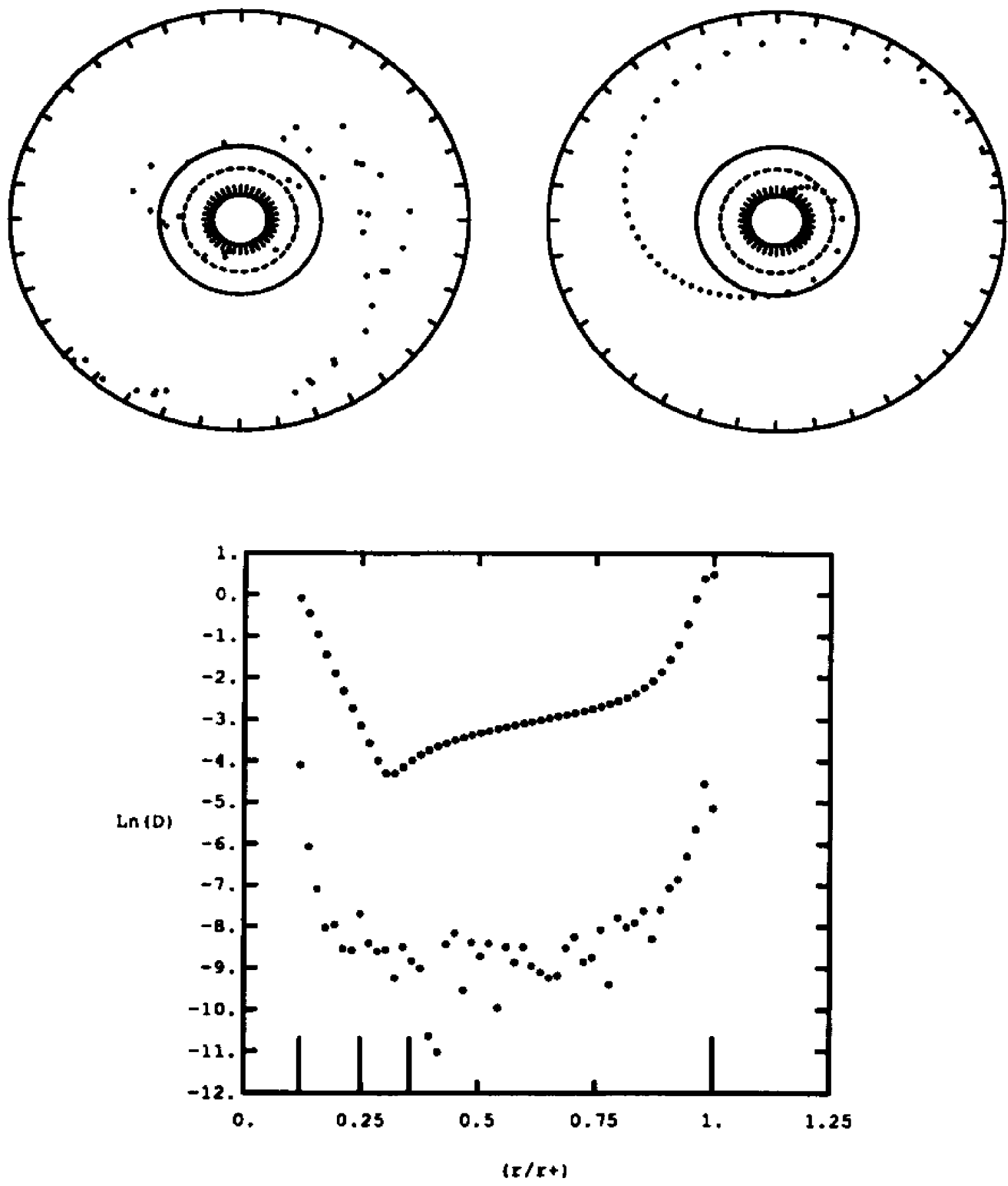

Figure 5.9b: The radial eigenfunction $\left(\phi_{1}-r\right)$ and amplitude as a function of radius $\left(D_{1}-r\right)$ of the $m=1$ Fourier mode illustrated as in Figure 2.3b for $M_{d} / M_{c}=1.0$, model \#2. 

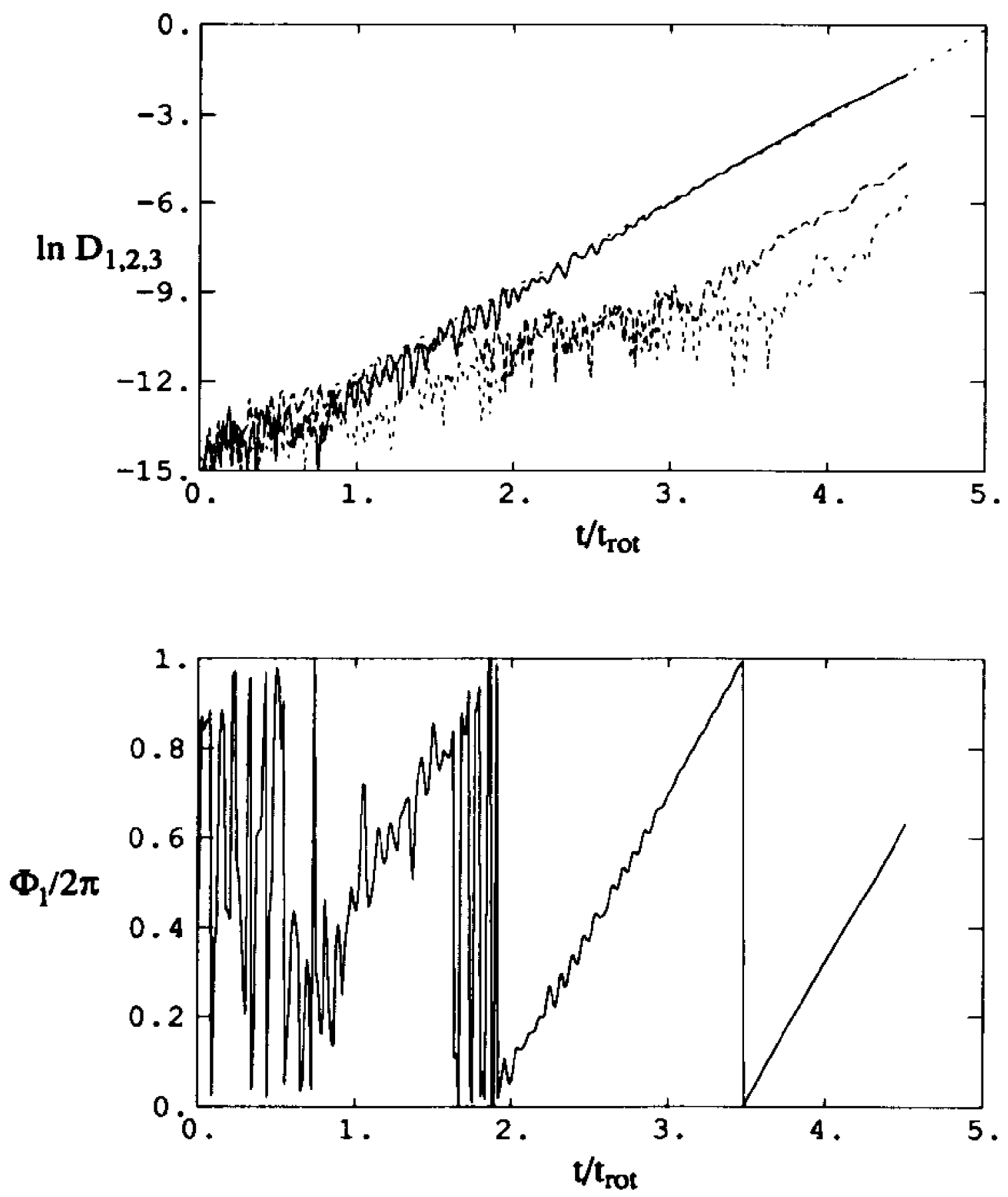

Figure 6.10a: The $m=1, m=2$, and $m=3$ Fourier amplitude behavior, and the $m=1$ phase behavior as a function of time, illustrated as in Figure 2.38 for $M_{d} / M_{\mathrm{c}}=1.0$, model \#3. 

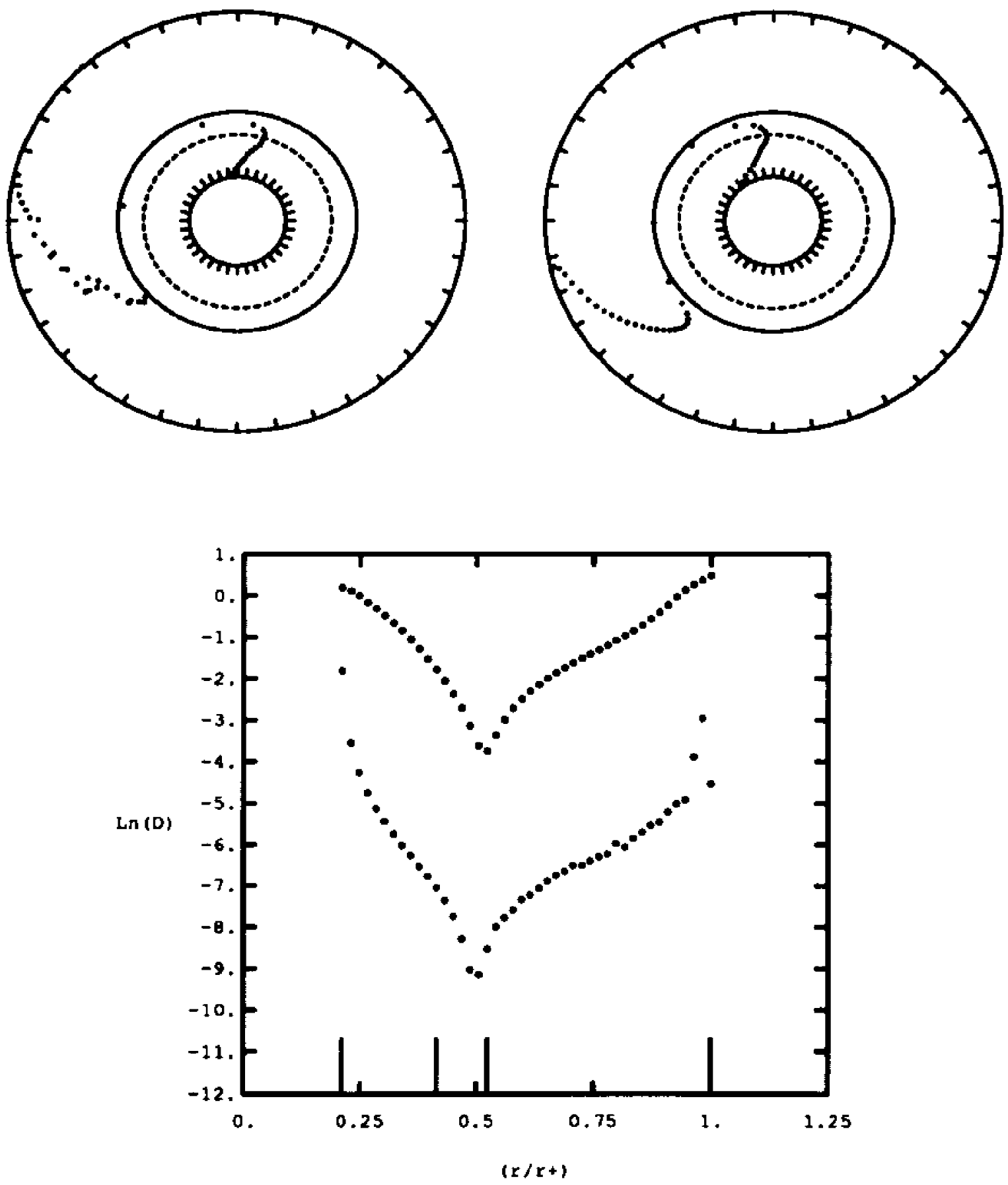

Figure 5.10b: The radial eigenfunction $\left(\phi_{1}-r\right)$ and amplitude as a function of radius $\left(D_{1}-r\right)$ of the $m=1$ Fourier mode illustrated as in Figure $2.3 \mathrm{~b}$ for $M_{d} / M_{c}=1.0$, model \#3. Note the $\pi / 2$ phase shift which is characteristic of cold $M_{d} / M_{c}=1.0$ models. 

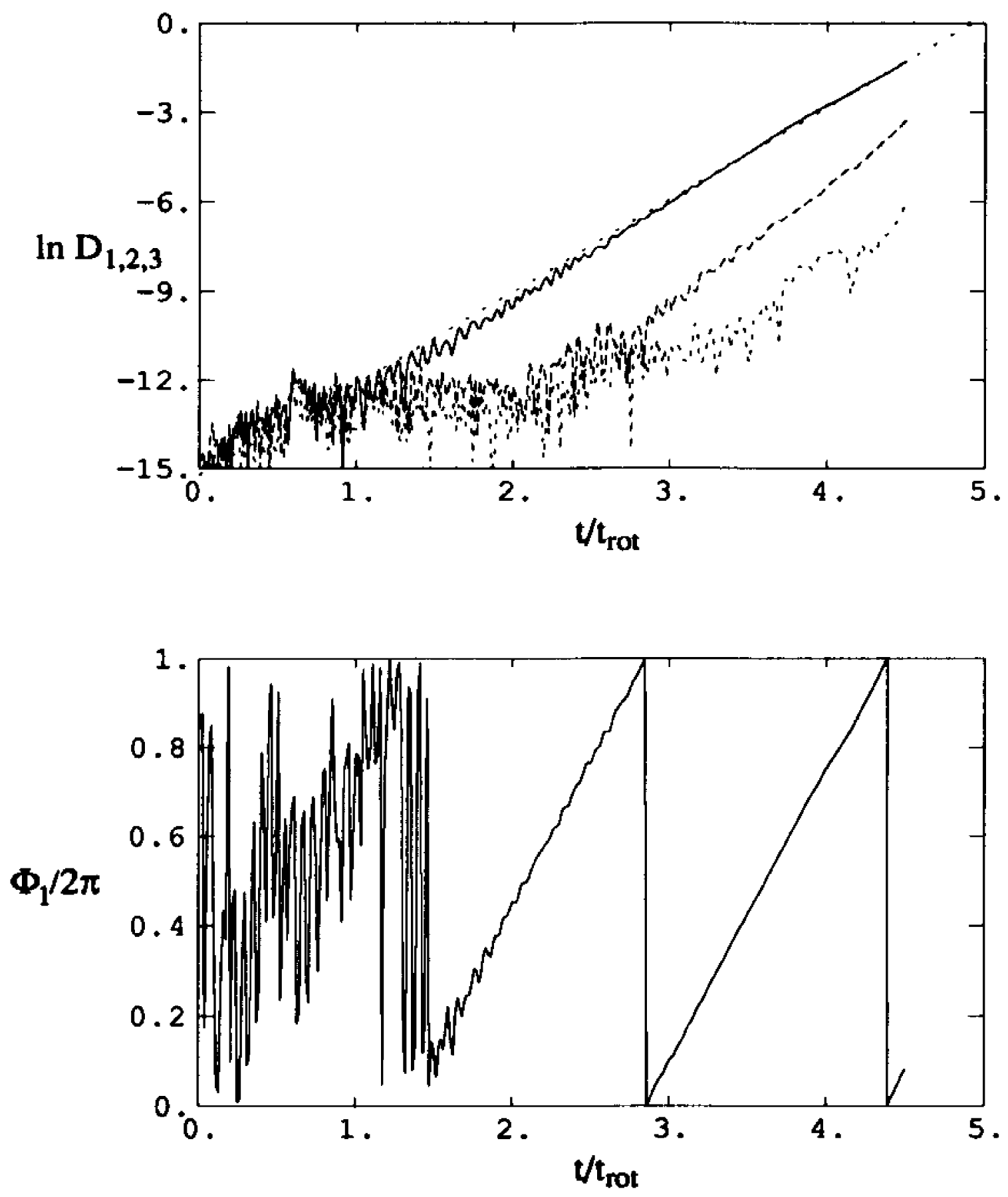

Figure 5.11a: The $m=1, m=2$, and $m=3$ Fourier amplitude behavior, and the $m=1$ phase behavior as a function of time, illustrated as in Figure 2.3a for $M_{d} / M_{c}=1.0$, model \#4. 

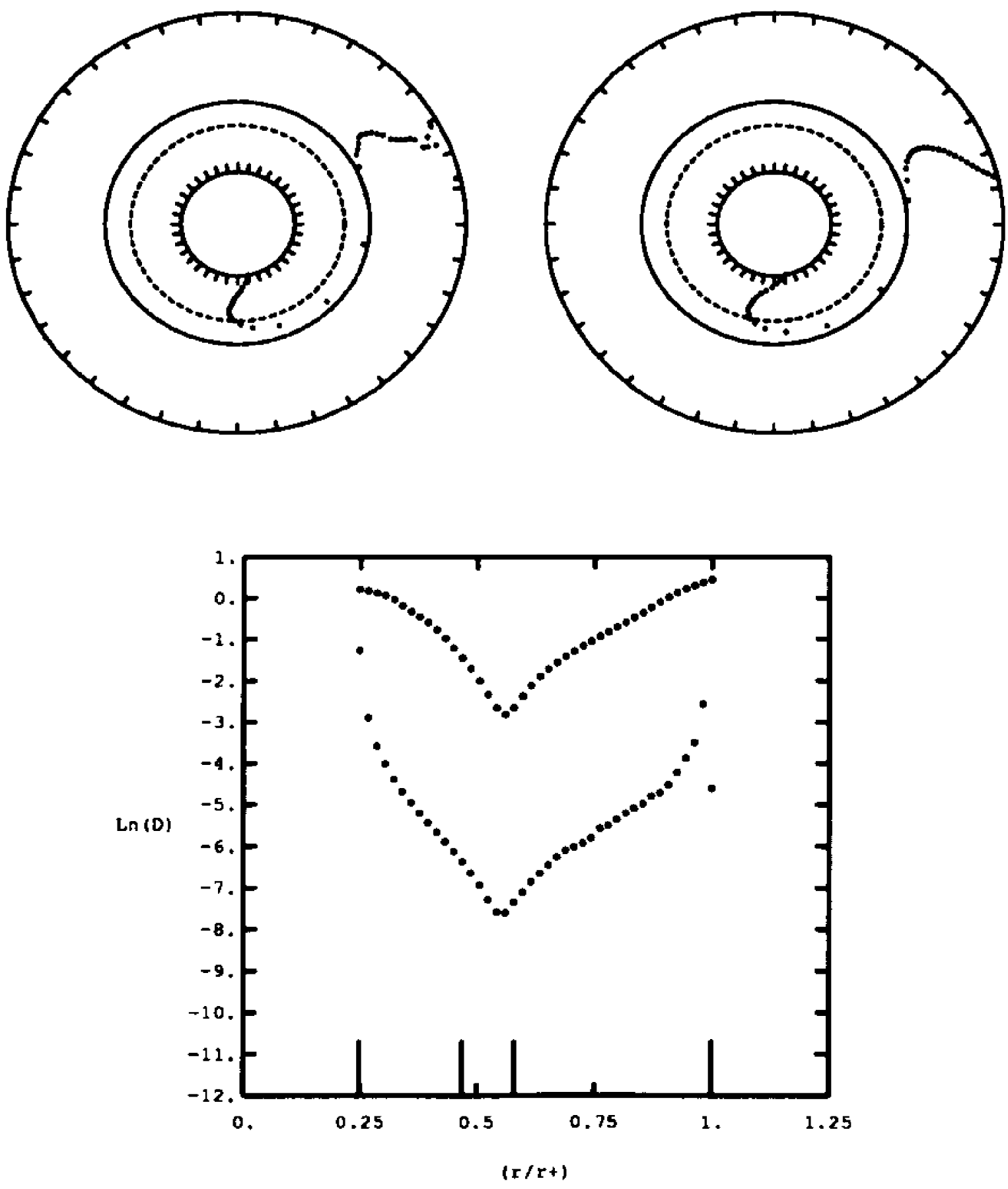

Figure 5.11b: The radial eigenfunction $\left(\phi_{1}-r\right)$ and amplitude as a function of radius $\left(D_{1}-r\right)$ of the $m=1$ Fourier mode illustrated as in Figure 2.3b for $M_{d} / M_{c}=1.0$, model \#5. 

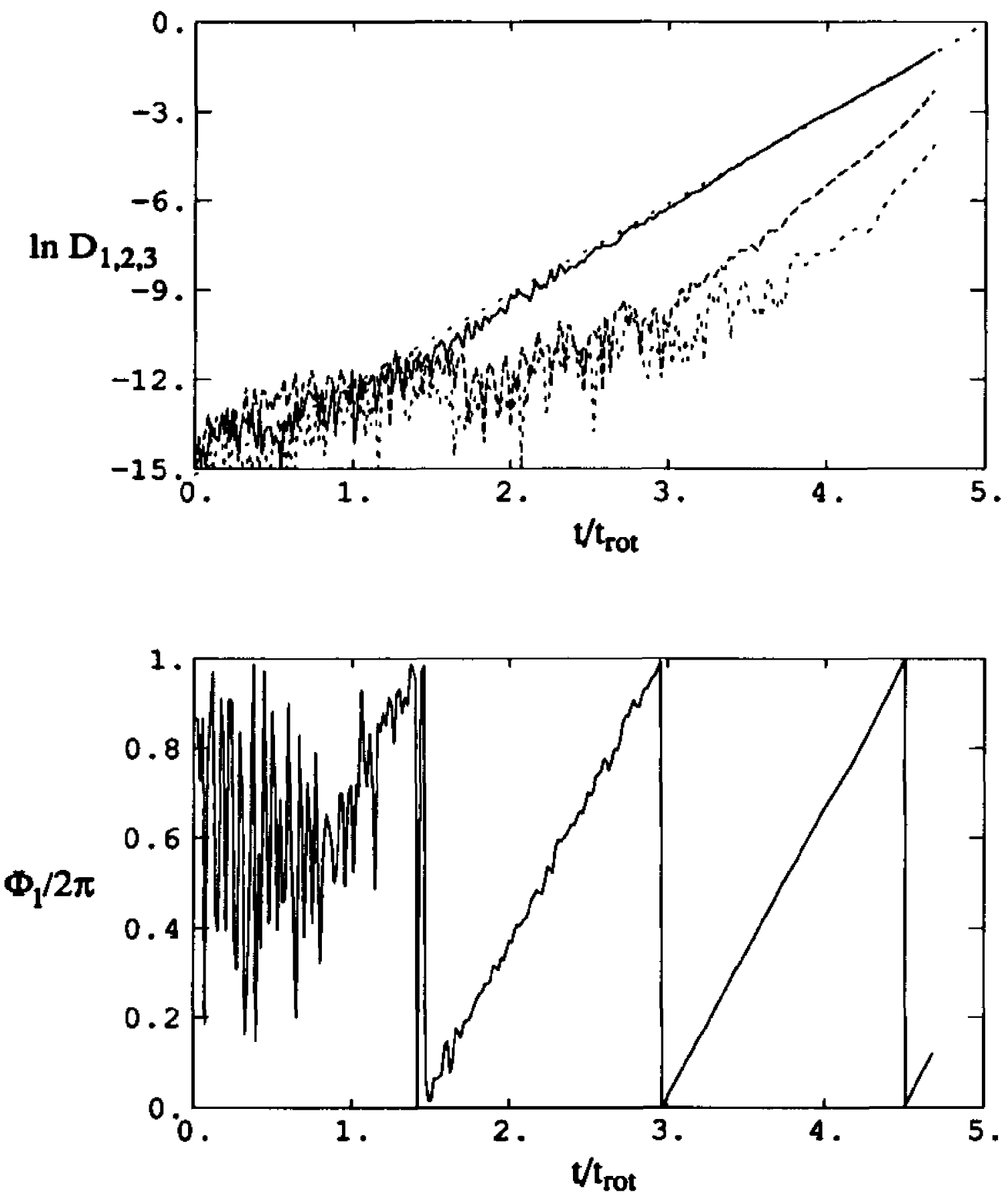

Figure 8.12a: The $m=1, m=2$, and $m=3$ Fourier amplitude behavior, and the $m=1$ phase behavior as a function of time, illustrated as in Figure 2.3a for $M_{d} / M_{\mathrm{c}}=1.0$, model \#5. 

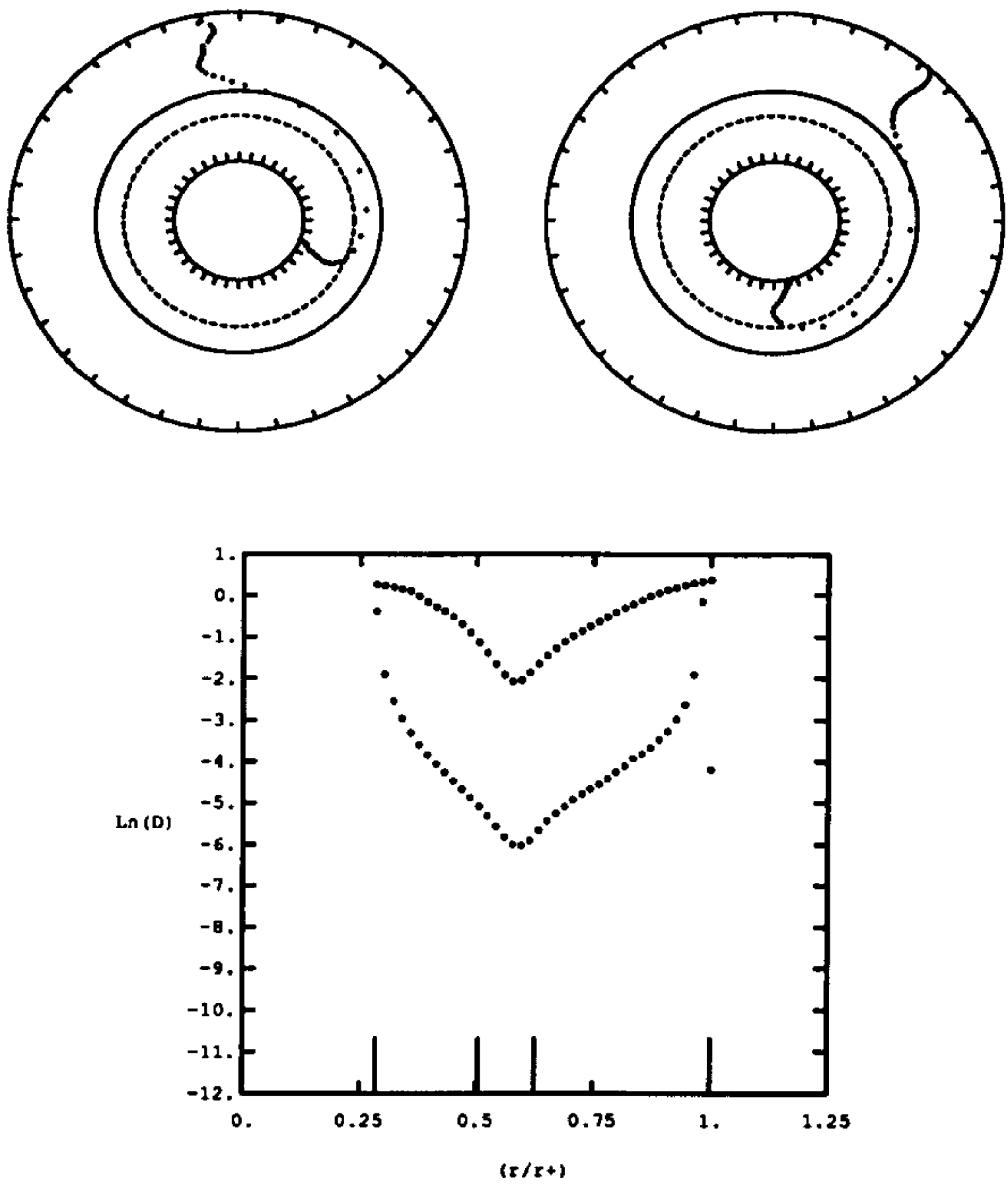

Figure 6.12b: The radial eigenfunction $\left(\phi_{1}-r\right)$ and amplitude as a function of radius $\left(D_{1}-r\right)$ of the $m=1$ Fourier mode illustrated as in Figure 2.3b for $M_{d} / M_{c}=1.0$, model \#5. 

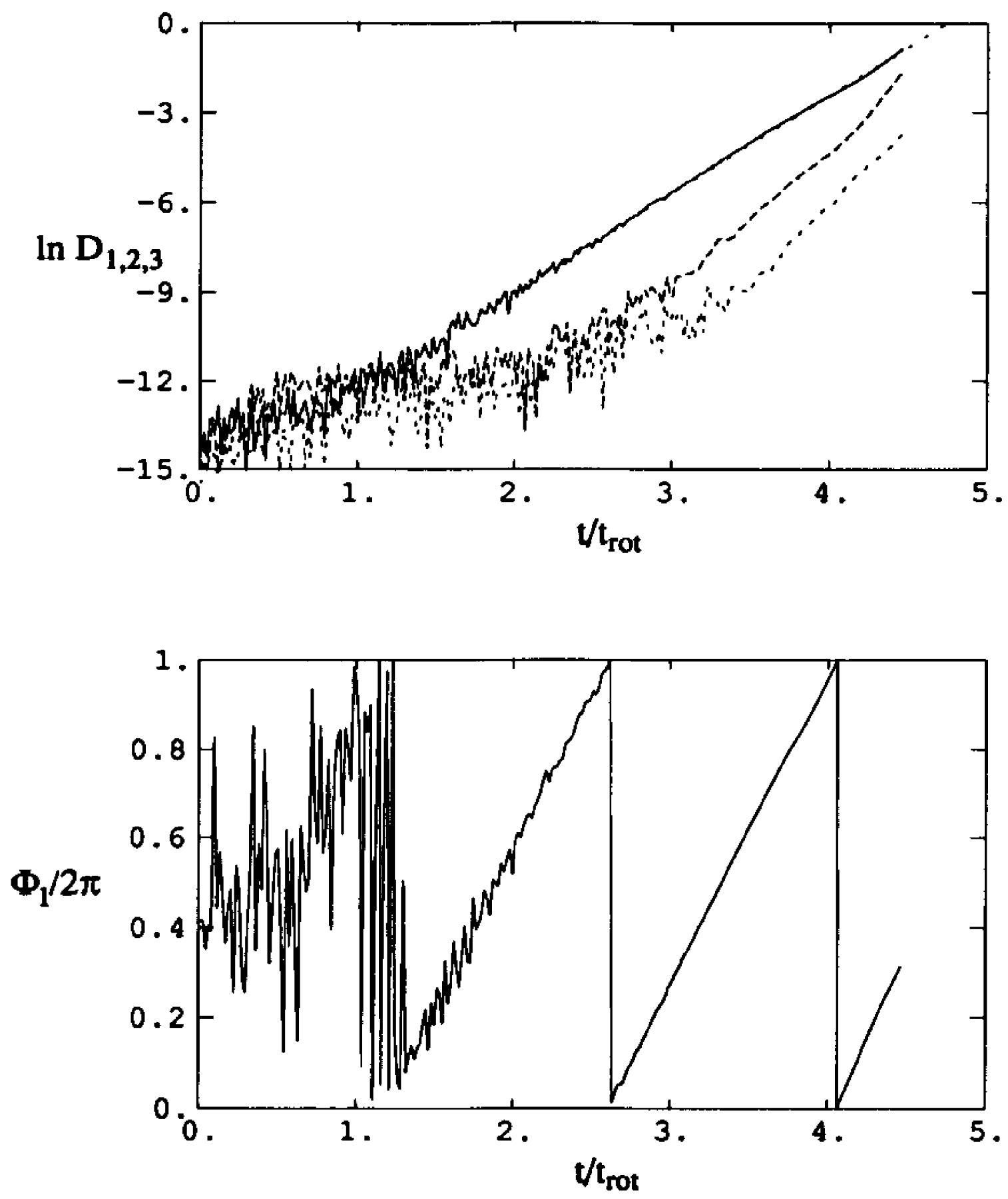

Figure 5.13a: The $m=1, m=2$, and $m=3$ Fourier amplitude behavior, and the $m=1$ phase behavior as a function of time, illustrated as in Figure 2.3a for $M_{d} / M_{c}=1.0$, model \#6. 

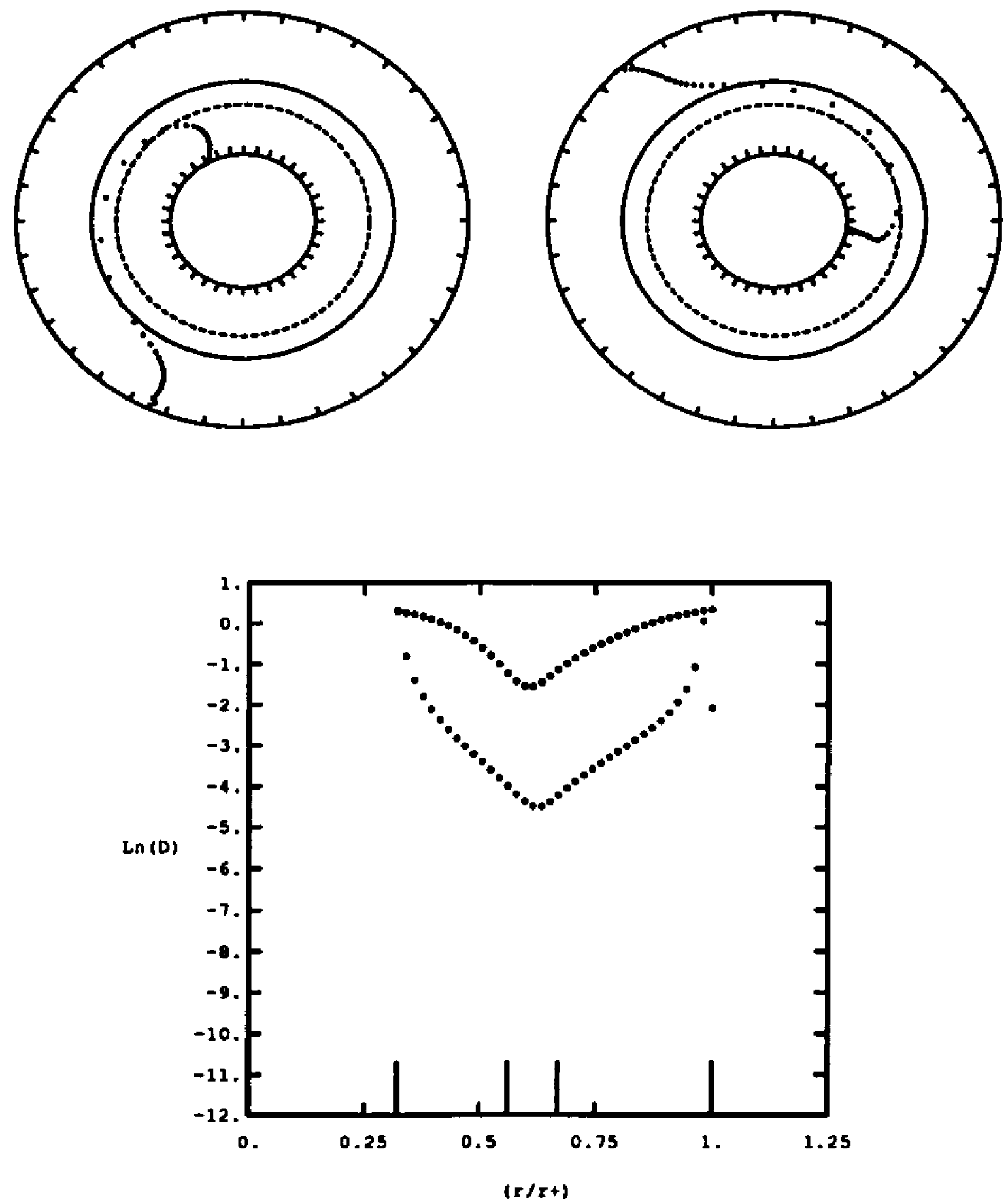

Figure 5.13b: The radial eigenfunction $\left(\phi_{1}-r\right)$ and amplitude as a function of radius $\left(D_{1}-r\right)$ of the $m=1$ Fourier mode illustrated as in Figure 2.3b for $M_{d} / M_{c}=1.0$, model \#6. 


\subsubsection{Systems with $M_{d} / M_{c}=0.2$}

Examination of the $y_{2}(1)$ parameters for models with mass ratio $M_{d} / M_{c}=0.2$ reveals the critical value of $T /|W|$ to be 0.26. Examination of Figures $5.15-5.18$ reveals that these models show the same $\phi_{1}-r$ behavior as the with mass ratio 1.0 - hot disks develop a spiral $m=1$ instability, whereas cooler disks undergo an instability characterized by a $\pi / 2$ phase shift in the leading direction. Figure $5.17 \mathrm{~b}$ indicates that model \#3 exhibits a nearly constant $\phi$ inside $r_{\mathrm{er}}$, a phase shift of $\pi$ occurring near that point, and a spiral $m=1$ behavior beyond that point. Model \#4 exhibits the curious $\pi / 2$ phase shift identical to the corresponding $M_{d} / M_{c}=1.0$ models and identified in conjunction with the P-mode instability (Appendix D). This leads us to propose that model \#3 is near a transition from one type of instability to the next. For the spiral mode, the pattern speed parameter clusters about $y_{1}(1) \approx-1 / 2$, whereas the other, less understood instability is determined to have $y_{1}(1)=-0.4$, not significantly different from the value determined for the corresponding $M_{d} / M_{c}=1.0$ models.

The $D_{1}-t$ figures reveal that models \#1 and \#2 may be exhibiting the Landau supercritical stability effect demonstrated by the $M_{d} / M_{c}=0.2$ models in $\$ 3.2 .3$ a clear initial growth of the $D_{1}$ parameter followed by a decrease in the rate of growth. In the case of model \#1, the growth is essentially halted entirely (the uncertainty of the measured growth rate is reflected by placing the $y_{2}(1)$ parameter in parentheses in Table 5.6), whereas model \#2 continues to grow, albeit at a much slower rate. The remaining models also demonstrate a shift in growth rate, but as the initial growth occurs over a smaller time period, the significance of the change is not as obvious. 
Table 5.5

Initial Model Data for $M_{d} / M_{c}=0.2$

\begin{tabular}{cccc}
\hline \hline$\overline{M o d e l ~ \#}$ & $r_{-} / r_{+}$ & $r_{0} / r_{+}$ & $T /|W|$ \\
1 & 0.074 & 0.139 & 0.261 \\
2 & 0.092 & 0.176 & 0.284 \\
3 & 0.148 & 0.268 & 0.335 \\
4 & 0.241 & 0.398 & 0.388 \\
\hline
\end{tabular}


Table 5.6

$\mathrm{m}=1$ Eigenmode Character, $M_{d} / M_{c}=0.2$

\begin{tabular}{cccccc}
\hline \hline Model & $y_{1}$ & $y_{2}$ & $\Omega_{p} / \Omega_{0}$ & $r_{c r} / r_{0}$ & $r_{c r} / r_{+}$ \\
1 & -0.65 & $(0.009)$ & 0.35 & 1.68 & 0.23 \\
2 & -0.49 & 0.026 & 0.51 & 1.39 & 0.24 \\
3 & -0.45 & 0.10 & 0.55 & 1.35 & 0.36 \\
4 & -0.41 & 0.25 & 0.59 & 1.30 & 0.52 \\
\hline
\end{tabular}



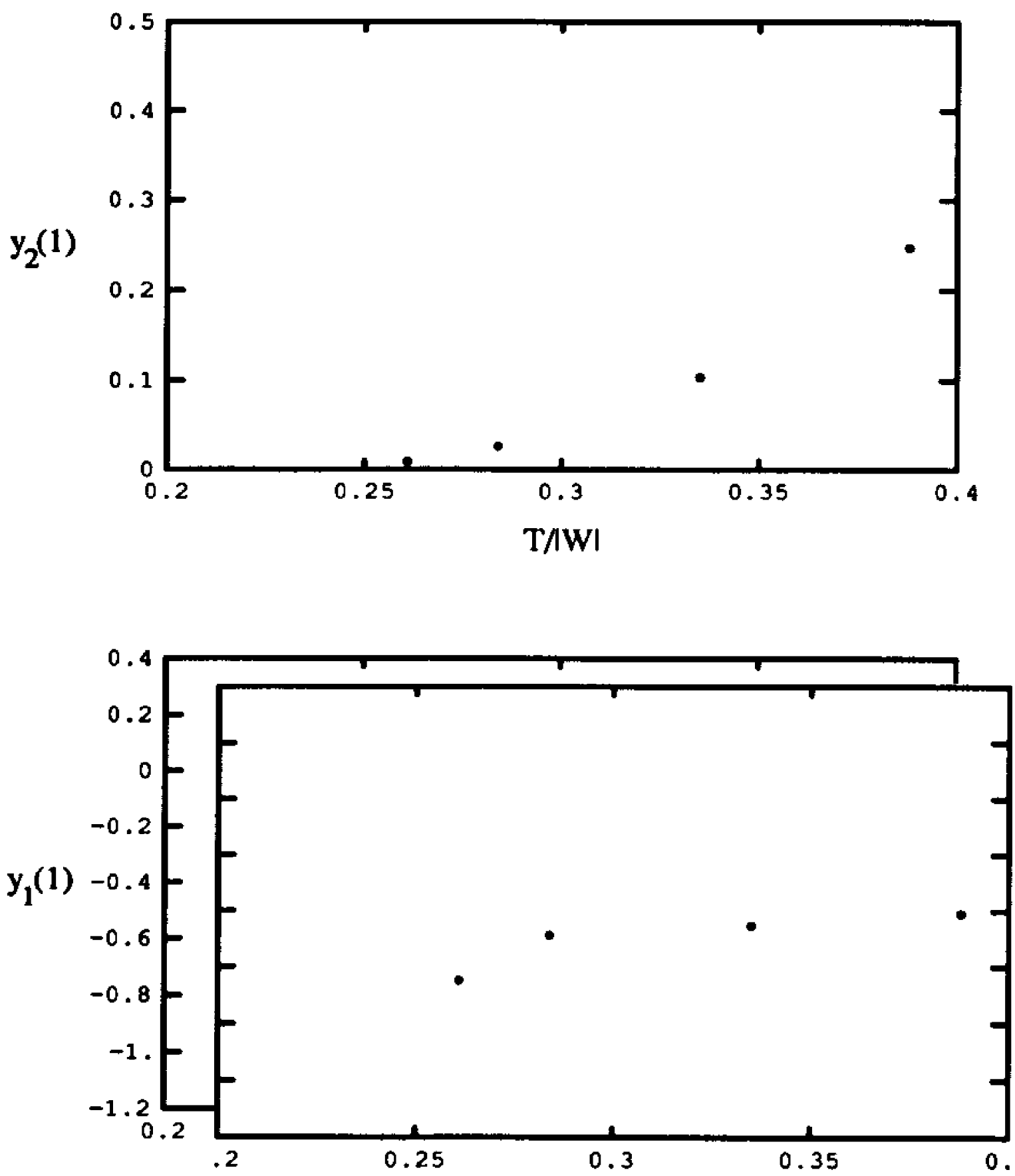

$\mathrm{T} / \mathrm{W} \mid$

Figure 5.14: $y_{2}(1)$ and $y_{1}(1)$ as functions of $T /|W|$ for $M_{d} / M_{c}=0.2$ models. 

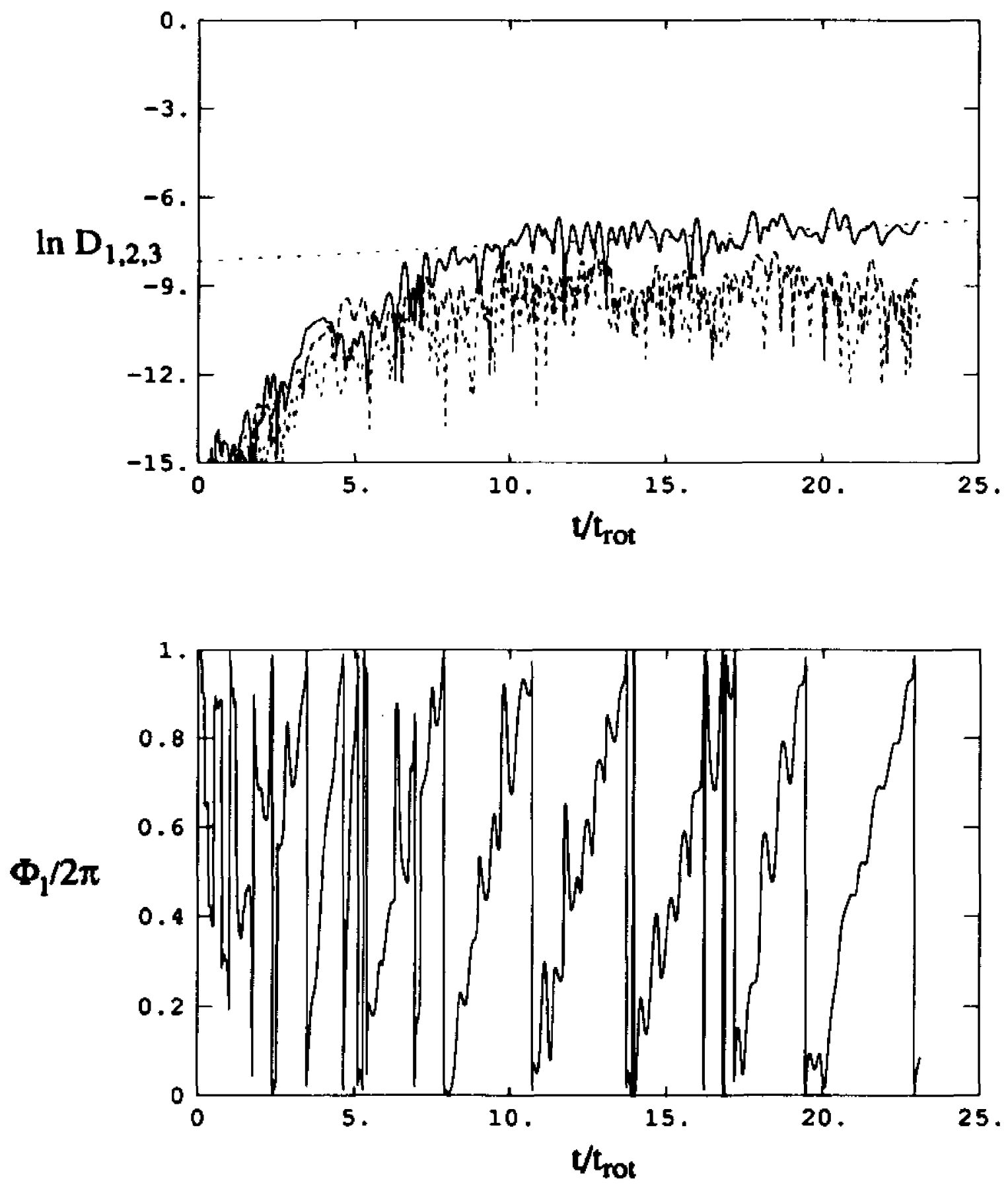

Figure 5.16a: The $m=1, m=2$, and $m=3$ Fourier amplitude behavior, and the $m=1$ phase behavior as a function of time, illustrated as in Figure 2.3a for $M_{d} / M_{c}=0.2$, model \#1. 

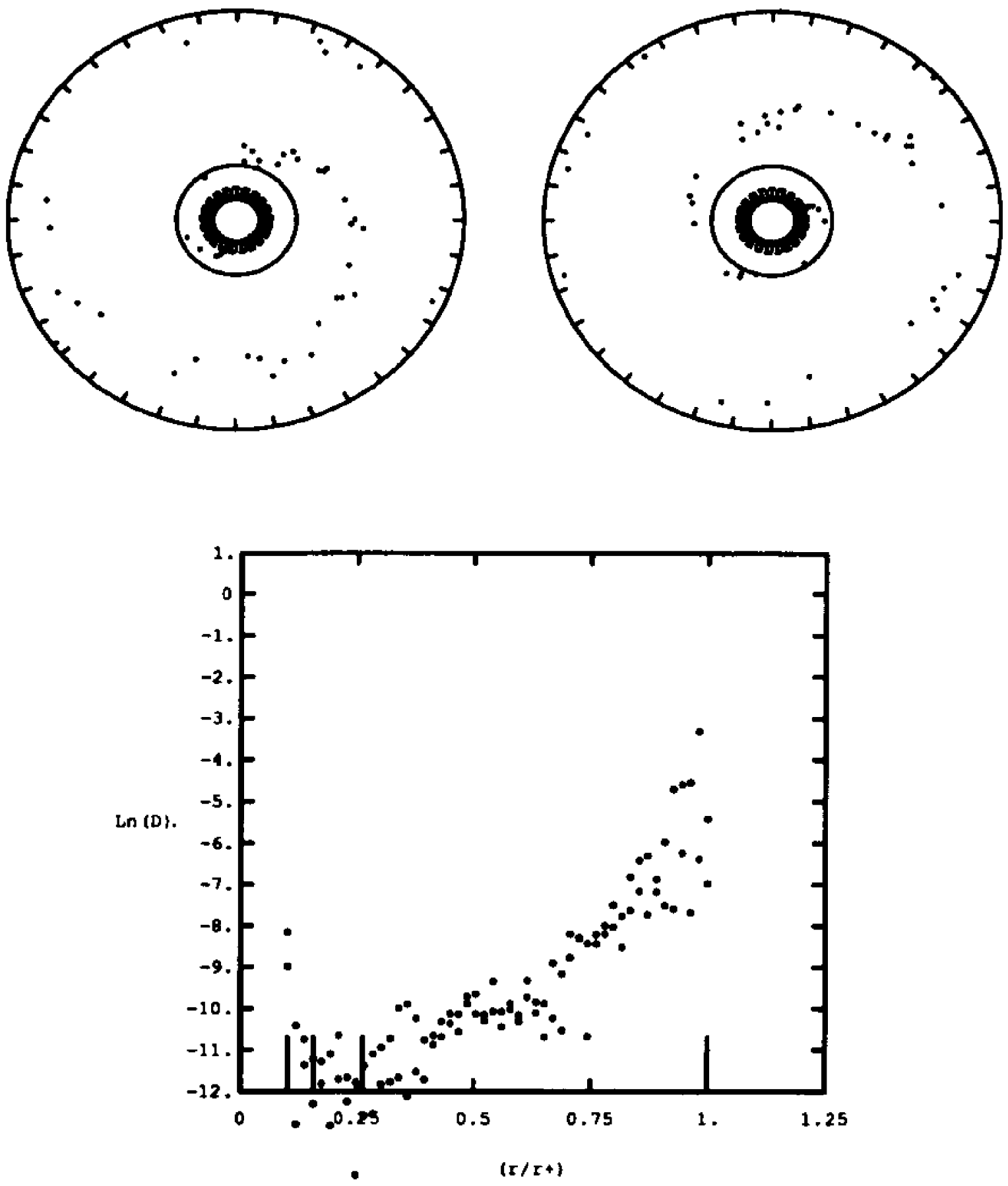

Figure 5.16b: The radial eigenfunction $\left(\phi_{1}-r\right)$ and amplitude as a function of radius $\left(D_{1}-r\right)$ of the $m=1$ Fourier mode illustrated as in Figure 2.3b for $M_{d} / M_{c}=0.2$, model \#1. 

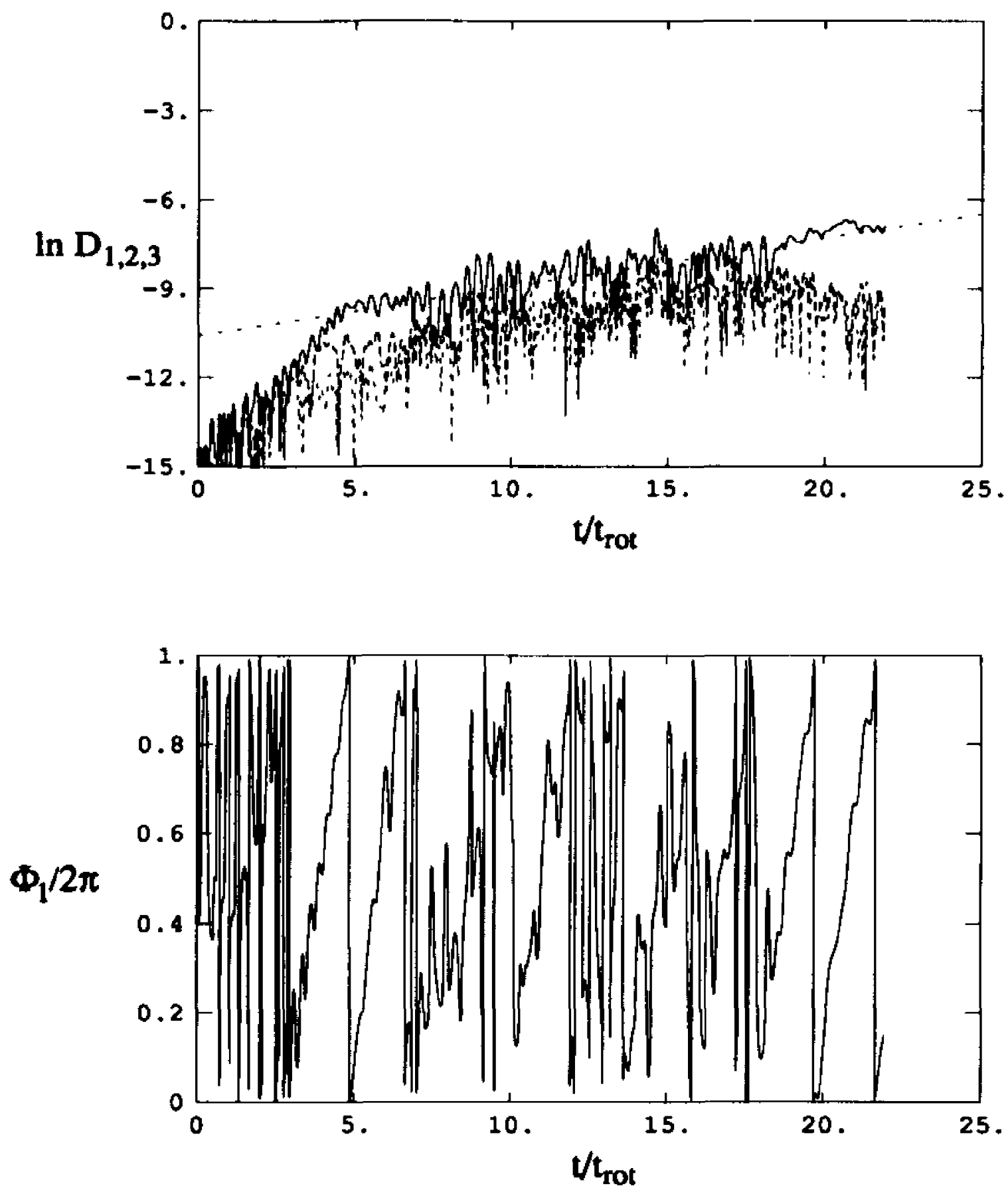

Figure 5.16a: The $m=1, m=2$, and $m=3$ Fourier amplitude behavior, and the $m=1$ phase behavior as a function of time, illustrated as in Figure 2.3a for $M_{d} / M_{\mathrm{c}}=0.2$, model \#2. 

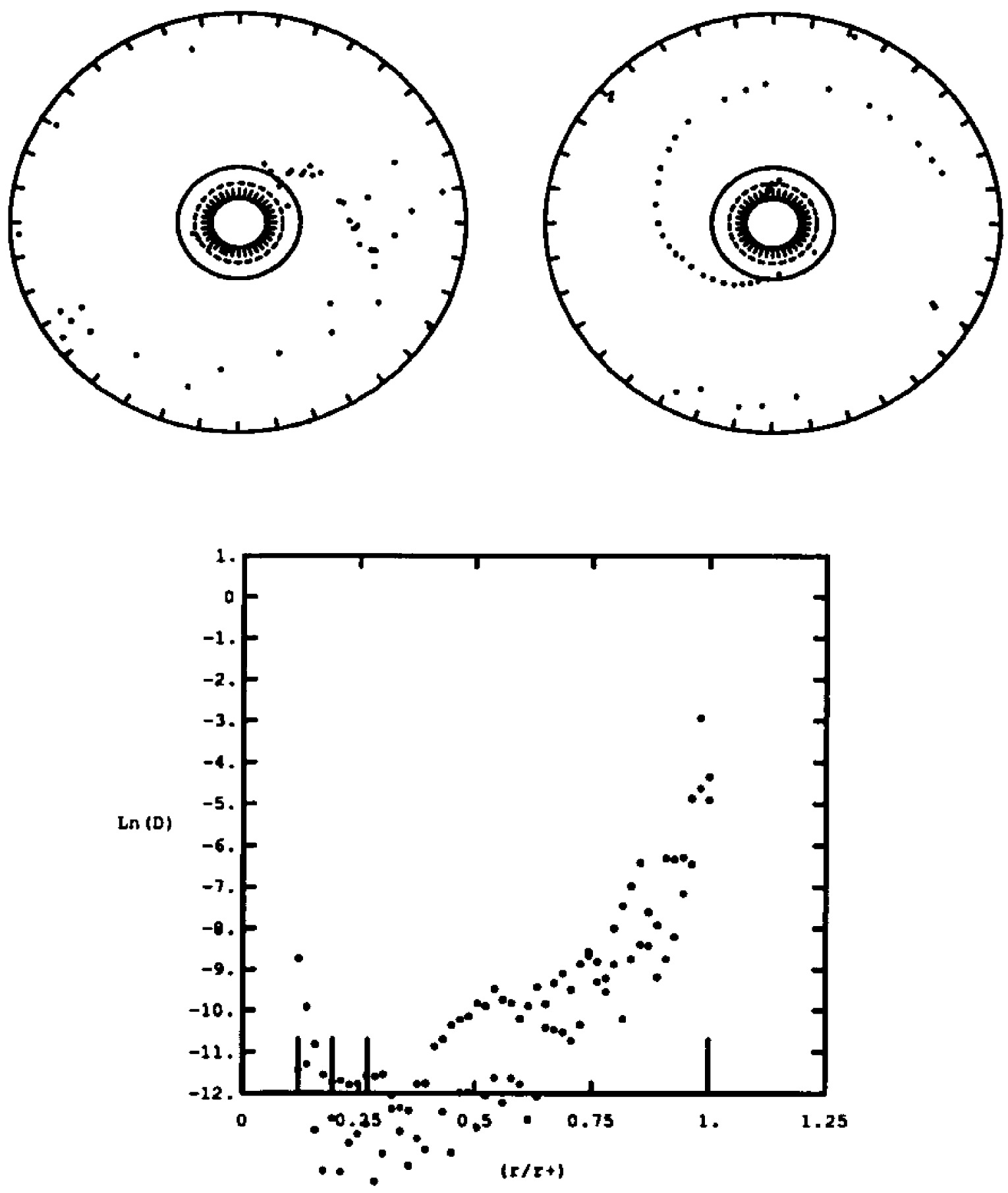

Figure 6.16b: The radial eigenfunction $\left(\phi_{1}-r\right)$ and amplitude as a function of radius $\left(D_{1}-r\right)$ of the $m=1$ Fourier mode illustrated as in Figure 2.3b for $M_{d} / M_{c}=0.2$, model \#2. 

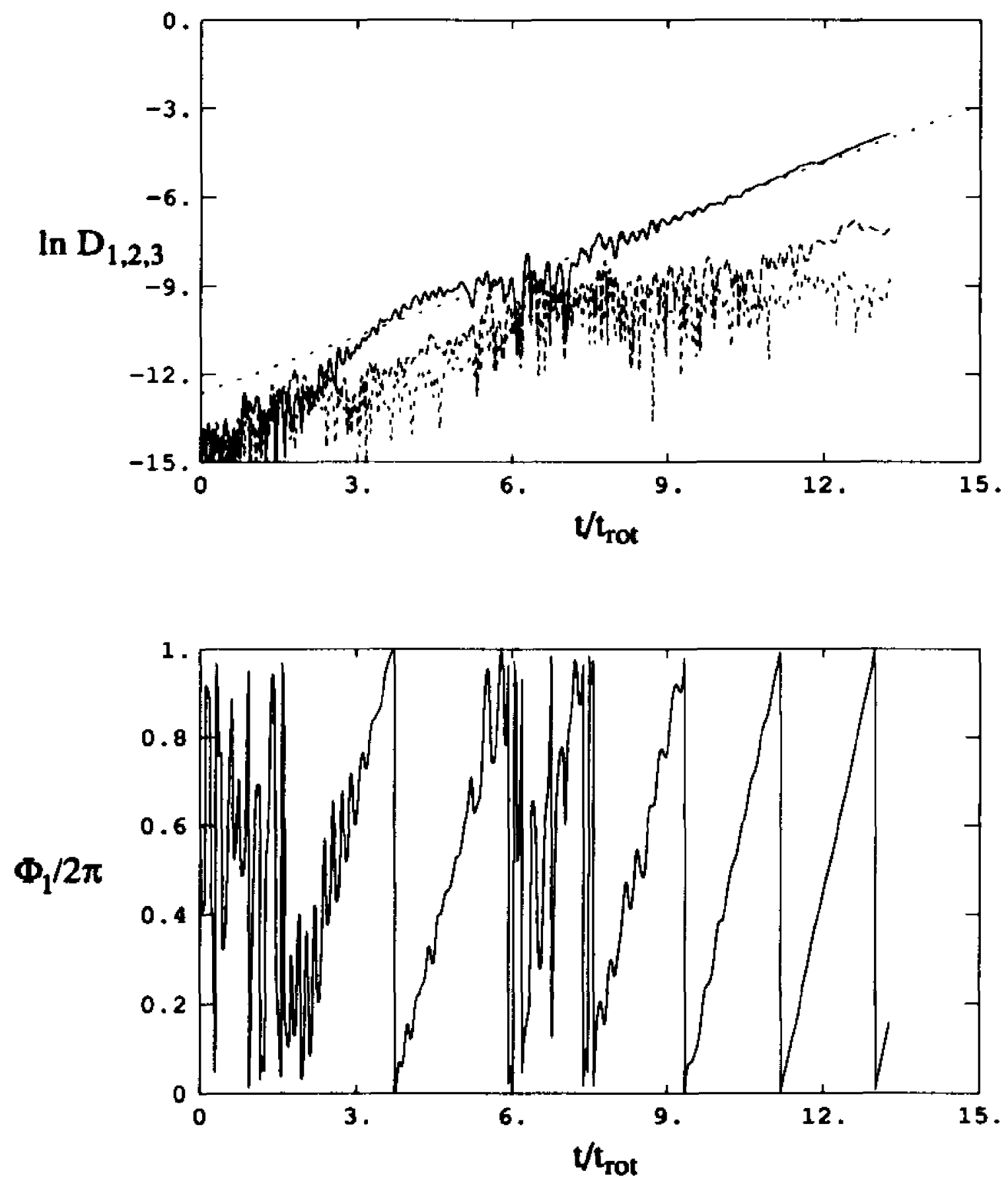

Figure 5.17a: The $m=1, m=2$, and $m=3$ Fourier amplitude behavior, and the $m=1$ phase behavior as a function of time, illustrated as in Figure 2.3a for $M_{d} / M_{c}=0.2$, model \#3. 

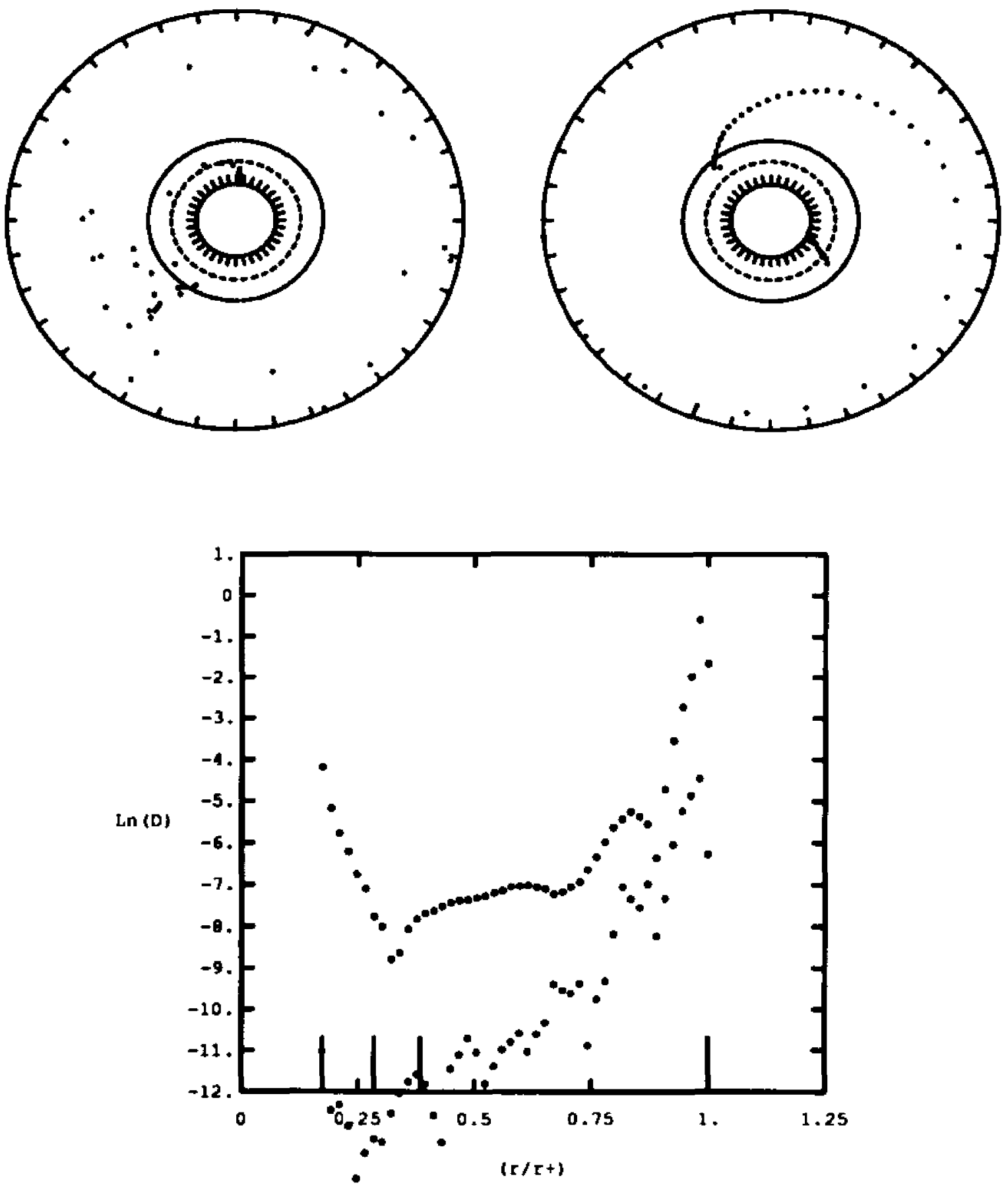

Figure 5.17b: The radial eigenfunction $\left(\phi_{1}-r\right)$ and amplitude as a function of radius $\left(D_{1}-r\right)$ of the $m=1$ Fourier mode illustrated as in Figure 2.3b for $M_{d} / M_{c}=0.2$, model \#3. 

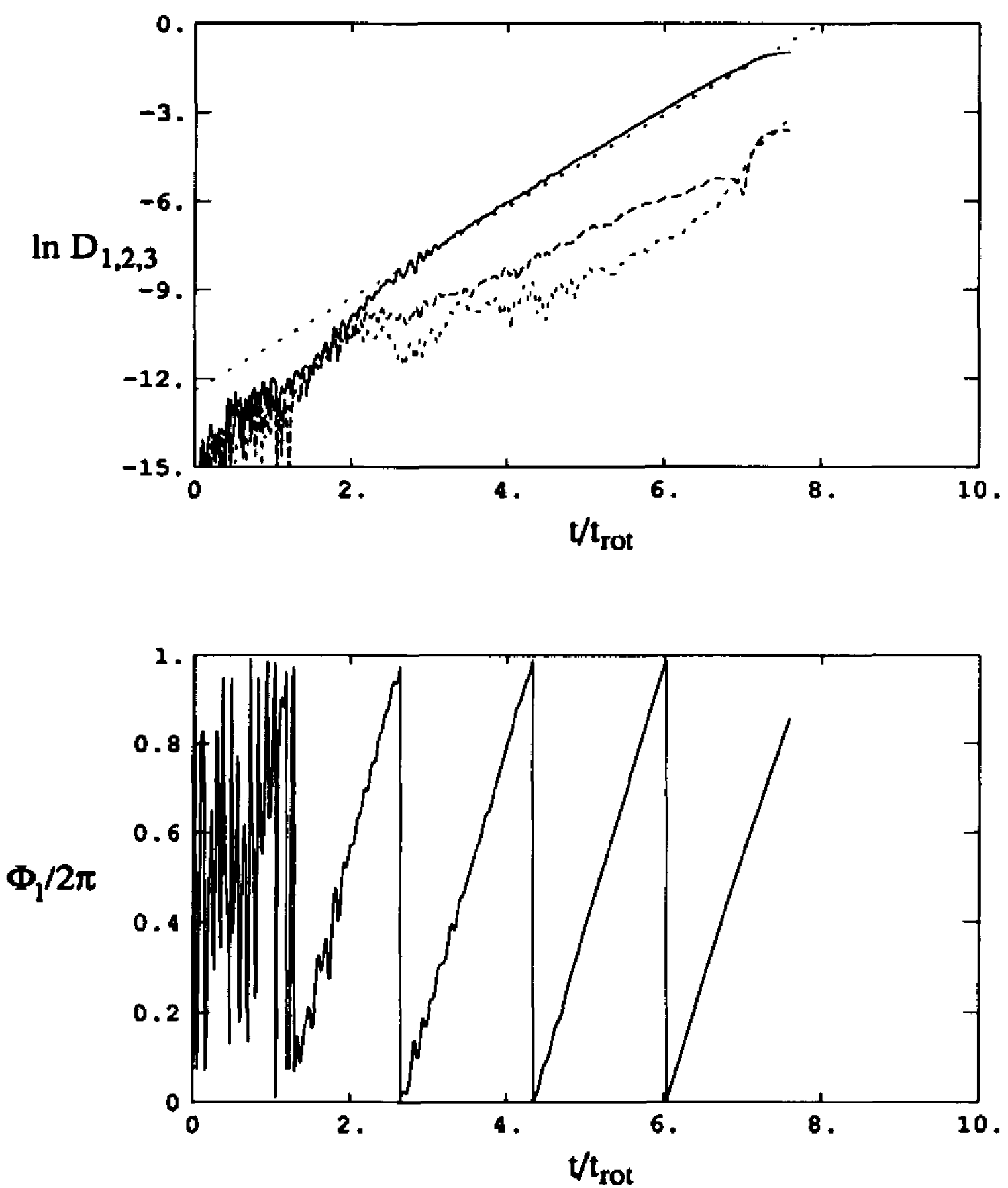

Figure 5.18a: The $m=1, m=2$, and $m=3$ Fourier amplitude behavior, and the $m=1$ phase behavior as a function of time, illustrated as in Figure 2.3a for $M_{d} / M_{c}=0.2$, model \#4. 

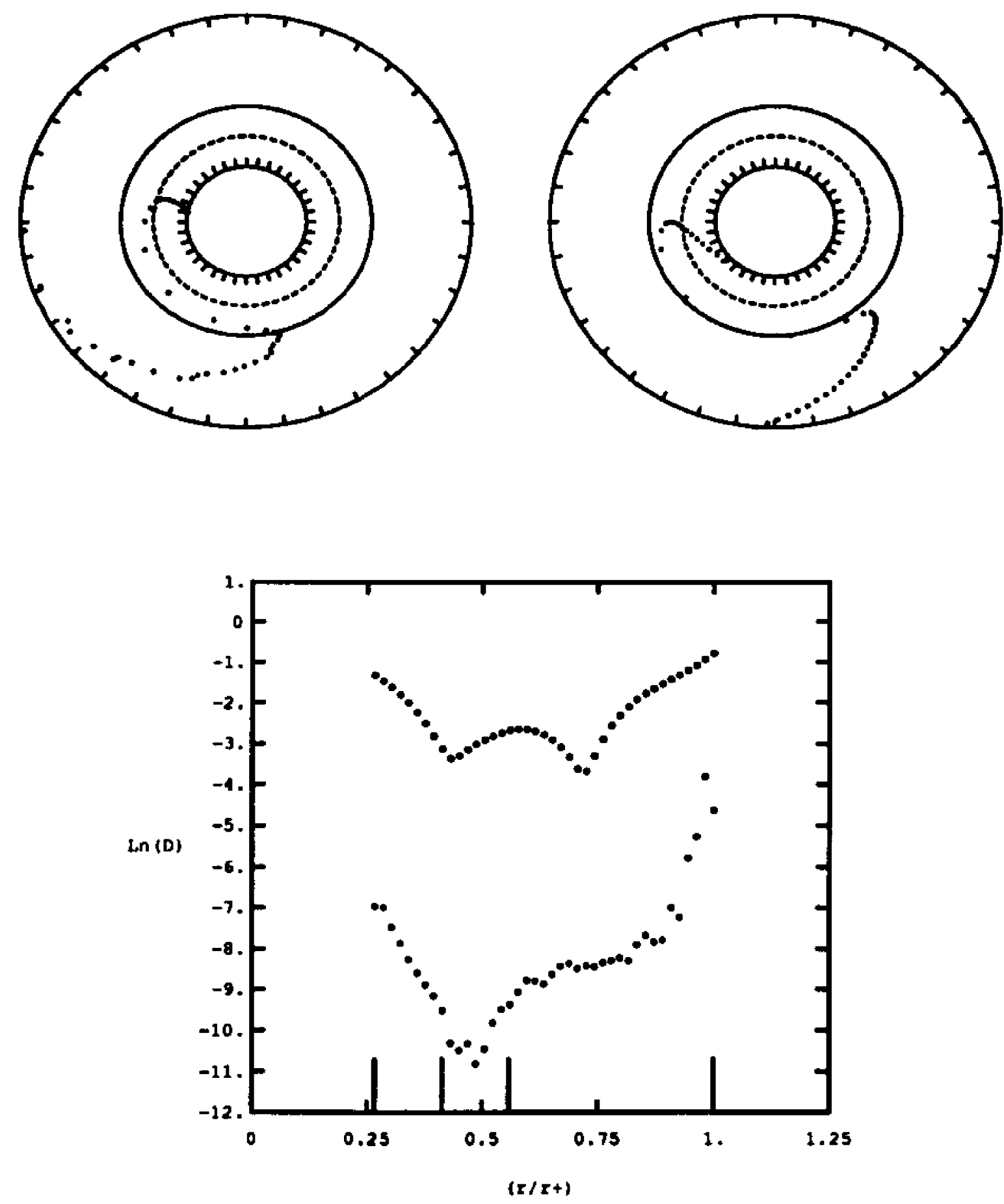

Figure 5.18b: The radial eigenfunction $\left(\phi_{1}-r\right)$ and amplitude as a function of radius $\left(D_{1}-r\right)$ of the $m=1$ Fourier mode illustrated as in Figure 2.3b for $M_{d} / M_{c}=0.2$, model \#4. 


\subsection{Summary of Odd-mode Evolutions}

As indicated in the upper panel of Figure 5.19, to the limits of our ability to measure $y_{2}(1)$, the critically unstable disks of mass ratio 5.0 (boxes), mass ratio 1.0 (open circles), and mass ratio 0.2 (solid circles) have values of $T /|W|=0.10$, $T /|W|=0.21$, and $T /|W|=0.26$, respectively. Models of all three mass ratios near the critically unstable value of $T /|W|$ ("hotter" disks) show spiral behavior very similar to that predicted by two-dimensional linear theory (Adams et al., 1989; Shu et al., 1990), and discussed in $§ 5.3$. This instability is characterized by:

- Azimuthal mode $m=1$.

- $y_{1}(1) \approx-0.5$.

- The $\phi_{1}-r$ behavior presents itself as a broad, trailing spiral with disk material coalescing to one side of the center of mass of the system.

Models with higher values of $T /|W|$ ("cooler" disks) clearly undergo a different instability, the majority revealing a leading phase shift in the eigenfunction of $\pi / 2$ - the nature of which is not yet understood. This instability is characterized by:

- Azimuthal mode $m=1$.

- $y_{1}(1) \approx-1 / 3$.

- A $\pi / 2$ phase shift exists in the $\phi_{1}-r$ plots, but the shift is in the direction of rotation, opposite that of the phase shift observed in the first $m=2$ instability characterized above.

In addition, mass ratio 0.2 disks near the onset of instability exhibit an al. teration in their growth rate characteristic of the Landau supercritical stability discussed in \$3.2.3. The instability exhibiting the leading $\pi / 2$ phase shift exhibits a $y_{1}$ (1) parameter similar to the zero-mass disks of Kojima (1986; crosses in Figure 5.19), $y_{1}(1) \sim-1 / 3$. Figure 5.20 illustrates the $y_{2}(1)$ parameter of all the models 
examined in this chapter according to their placement on the $T /|W|$ vs. $M_{d} / M_{c}$ diagram. 

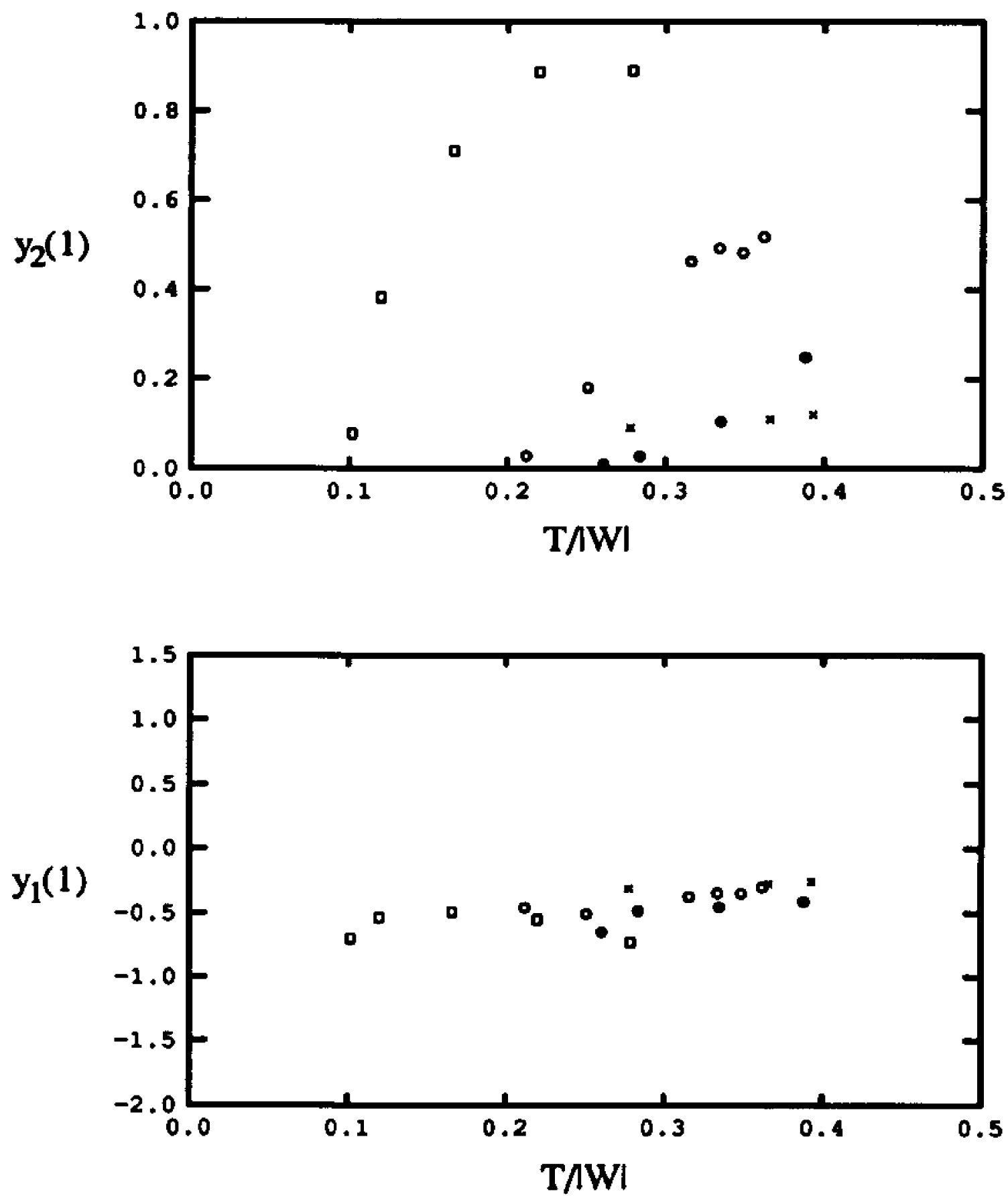

Figure 8.19: $y_{2}(1)$ and $y_{1}(1)$ as functions of $T /|W|$ for all models in which the $\pi$-symmetry constraint has been removed; mass ratios 5.0 (boxes), 1.0 (holiow circles), and 0.2 (solid circles). Crosses indicate linear analyais $M_{d} / M_{c}=0.0$ modelo with the lowest $y_{2}(1)$ by Kojima (1986). 


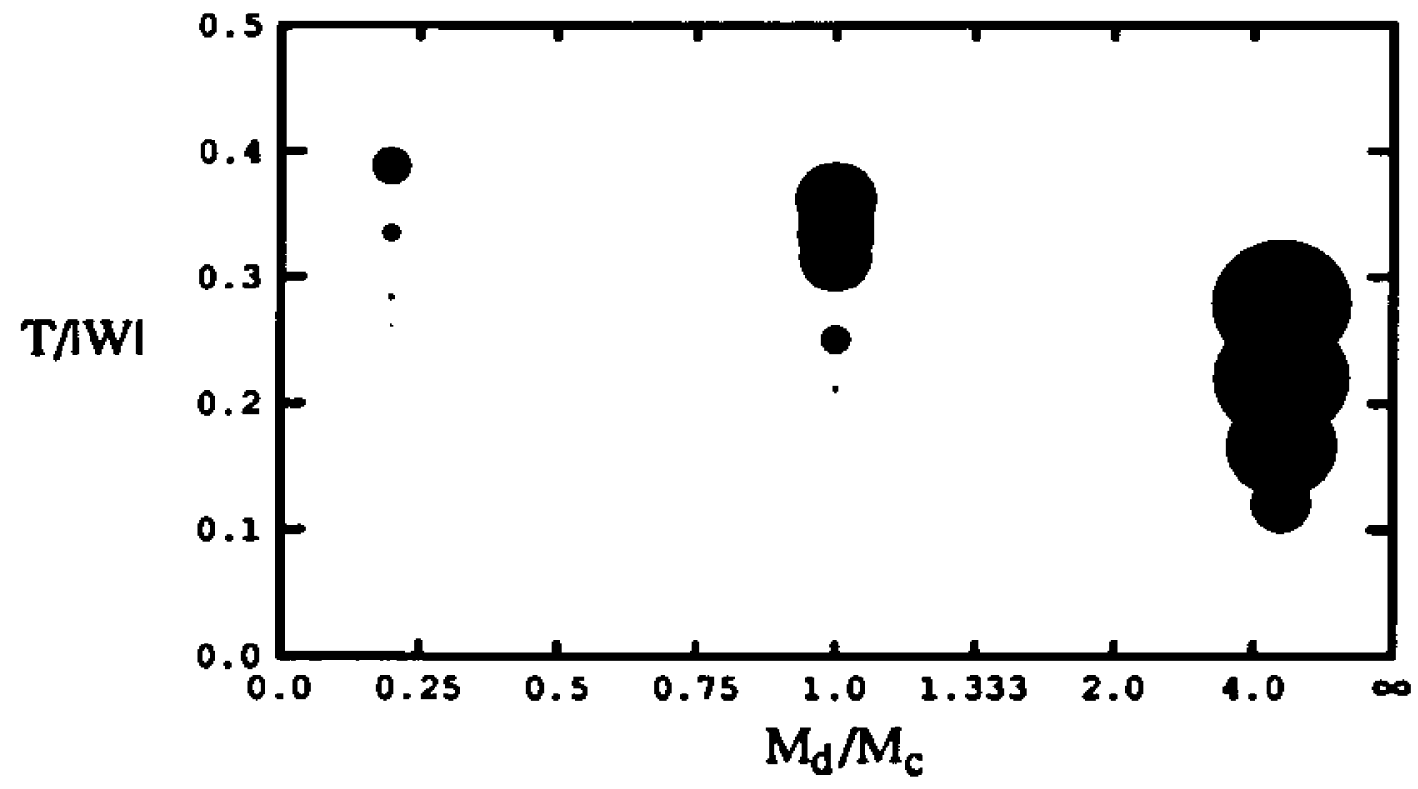

Figure 5.20: Growth rate results from all of the evolutions in which the $\pi$-symmetry constraint has been removed are plotted on a $T /|W|$ vs. $M_{d} / M_{c}$ template. The size of each circle is directly proportional to the growth rate parameter $y_{2}(2)$. 


\section{CHAPTER 6}

CONCLUSIONS

\subsection{Summary of Simulations}

From a careful examination of the $\mathbf{4 1}$ models presented in this study, a number of conclusions can be drawn. The first conclusion concerns itself with the hydrodynamic code that has been developed to study the stability of astrophysical accretion disk systems. Being second-order accurate in both space and time, the code is inberently more accurate than the first-order hydrodynamic code that was used in TH90. The lack of correction factors to the growth rate parameter allows the evolution of models which lie much closer to the critically unstable value of $T /|W|$. This is evidenced clearly through model \#1 of mass ratio $\infty$ where the first-order hydrocode results in a corrected growth rate parameter of $y_{2}(2)=0.136$, whereas the second-order code provides an uncorrected growth rate parameter $y_{2}(2)=0.079$. In addition, several models in this stability analysis were found to have growth rate parameters $y_{2}(2)$ approaching zero. In these cases, the numerical diffusion present in the first-order code would not have allowed any nonaxisymmetric structure to develop. This ability to examine systems with nearly zero growth rates more readily allows the identification and subsequent study of marginally unstable models.

The measured $y_{2}(m)$ parameter has been plotted in Figure 6.1 for 40 of the 41 evolved models (model \#6, $M_{d} / M_{c}=5.0$ from the $\pi$-symmetry evolutions has been omitted from the figure because of its large growth rate, $\left.y_{2}(2)=1.7\right)$. In this summary figure, which combines Figures 3.24 and 5.20, open circles indicate models in which $\pi$-gymmetry has been enforced (results from Chapters 2 \& 3); shaded circles indicate that odd modes have been allowed to grow (results from Chapter 5). 


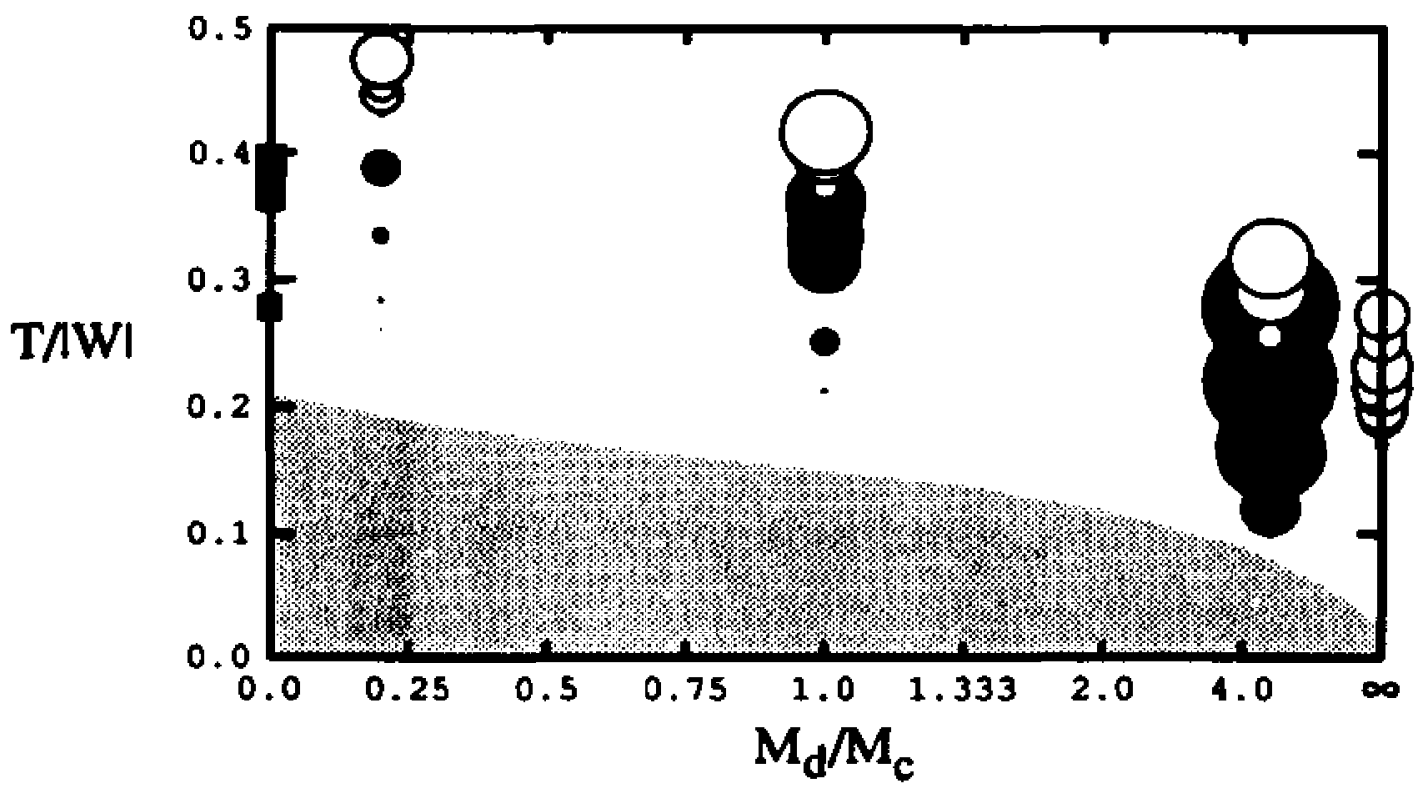

Figure 6.1: Summary of growth rates for all models evolved. Open circles indicate $\pi$-symmetry enforced; shaded circles indicate relaxation of $\pi$-8ymmetry constraint; shaded boxes indicate linear results of Kojima (1986). As explained in the caption to Figure 2.2, the shaded region across the lower portion of the diagram is inaccessible to the present study.

The radius of the circle depends linearly upon the growth rate parameter. For comparison at low mass ratios, the boxes drawn on the left-hand vertical axis of Figure 6.1 illustrate $m=1$ growth rates derived from the linear stability study of Kojima (1986). (Kojima's models are $n=3.0$ polytropes with $M_{d} / M_{c}=0$; the three depicted here have $T /|W|=0.278,0.366$, and 0.393 , and the corresponding growth rates are $y_{2}(1)=0.09,0.11$, and 0.12 .) The circles with the smallest diameters in Figure 6.1 pinpoint the locus of "marginally unstable" models. A smooth curve drawn through the marginally unstable models can now be used as the demarcation line separating stable systems from dynamically unstable ones in the $T /|W|-M_{d} / M_{c}$ plane. In general, systems with high mass ratios must have low values of $T /|W|$ (higher thermal support) in order to be stable, whereas systems of low mass ratio 
may be more rotationally supported (cooler; lower value of $T /|W|$ ) and remain dynamically stable. In addition, systems in which $\pi$-symmetry has been enforced are stable at higher values of $T /|W|$ than systems in which the symmetry constraint has been removed.

Dynamical instabilities exhibiting four different basic properties or behaviors have been observed in this study. The properties of each instability can be characterized (or distinguished) by: the pattern speed parameter, $y_{1}(m)$; the phase vs. radius $\left(\phi_{m}-r\right)$ behavior; and, in some cases, the time-variation of the amplitude of the dominant Fourier mode $\left(D_{m}-t\right)$. Enforcing $\pi-8 y m m e t r y, ~ s y s t e m s$ with mass ratios 5.0, and 1.0, as well as several of the mass ratio 0.2 systems, showed the same characteristic behavior as the instability which arises in the mass ratio $\infty$ systems first evolved in TH90:

- Azimuthal mode $m=2$.

- $y_{1}(2) \approx-1.0$, indicating that corotation is outside of $r_{0}$, but inside $r_{+}$.

- $\pi / 2$ phase shift near $r_{0}$.

- Higher order modes develop and eventually dominate if one examines sufficiently high $T /|W|$ (sufficiently "cold") models.

Another unstable mode emerged in mass ratio $0.2, \pi$-symmetry systems as $T /|W|$ was decreased:

- Azimuthal mode $m=2$.

- $y_{1}(2) \sim+0.1$ to +0.3 , and $r_{c r} \approx r_{0}$.

- $\phi_{2}$ vs. $r / r_{0}$ is coherent throughout (no $\pi / 2$ phase shift evident).

- $D_{2}$ vs. $t$ grows early in the evolution, but levels off at a low amplitude, reminiscent of Landau's "supercritical stability" behavior. 
Removal of the $\pi$-symmetry constraint revealed two new instabilities resulting from interactions between the disk and the central object. At all modeled mass ratios, the instability which arose near the critical value of $T /|W|$ is reminiscent of the instability first identified in a linear stability analysis by Adams et al. (1989). In our simulations, this instability is characterized by:

- Azimuthal mode $m=1$.

- $y_{1}(1) \approx-0.5$.

- The $\phi_{1}-r$ behavior presents itself as a broad, trailing spiral with disk material coalescing to one side of the center of mass of the system.

Somewhat cooler (higher $T /|W|$ ) disks exhibited an unstable mode with the following character:

- Azimuthal mode $m=1$.

- $y_{1}(1) \approx-1 / 3$.

- A $\pi / 2$ phase shift exists in the $\phi_{1}-r$ plots, but the shift is in the direction of rotation, opposite that of the phase shift observed in the first $m=2$ instability characterized above.

This fourth azimuthal eigenfunction does not lend itself to a simple geometric interpretation though we can tentatively identify the instability which exhibits in this eigenfunction as the Papaloizou-Pringle P-mode instability (Papaloizou \& Pringle 1984).

\subsection{The Relationship to Star Formation}

In what manner can the simple determination of a critical value of $T /|W|$ for a variety of system mass ratios be related to the formation of stellar systems? The key to such a relationship lies in the formation of the disk structure from the 
material present in a collapsing molecular cloud core. Drawing from the studies of the collapse of cloud cores by Cassen and Moosman (1981), Stemwedel, Yuan, and Cassen (1990), and simulations by Bodenheirner, Yorke, Różyczka, and Tohline (1990), we see that as cloud material falls onto the central object and the disk, a system with disk-to-central-object mass ratio on the order of unity eventually can form. The simulations by Bodenheimer $e t$ al. in particular indicate that initially a significant quantity of cloud material falls directly onto the central object in a short period of time. Subsequently, however, material falls more directly onto the disk structure. More specifically, at a time $t \sim 10$ years in the Bodenheimer et al. work, the central object has a mass $M_{c} \sim 0.5 M_{\odot}$, with little formation of a disk. Hence, at this early point in time, $M_{d} / M_{c}$ is essentially zero. As the disk begins to form, it is heated by an accretion shock, making $T /|W|$ initially quite low. This provides an initial placement on the $T /|W|$ vs. $M_{d} / M_{c}$ diagram (eg., point A in Figure 6.2). As time progresses and the rapid infall of high specific angular momentum material proceeds, the disk-to-central-object mass ratio increases while $T /|W|$ remains low due to the presence of the accretion shock. Thus, we can envision the evolution of the system through the $T /|W|$ vs. $M_{d} / M_{c}$ diagram as following a roughly horizontal path towards higher mass ratio. At the end of the Bodenheimer et al. simulation, the mass ratio has been well established as $\lesssim 1$, with the temperature of the central region decreasing due to the decreasing influence of radial accretion of disk material onto the central object, and the disk has a well-developed, axisymmetric equilibrium structure. The disk is expected to cool significantly as infall onto the disk dissipates. When this occurs, the position of the system in the $T /|W|$ vs. $M_{d} / M_{c}$ diagram should move upwards.

It is at this point that we see the relationship to the stability survey presented in this dissertation. The evolutionary path of the protostellar system in the $T /|W|$ 


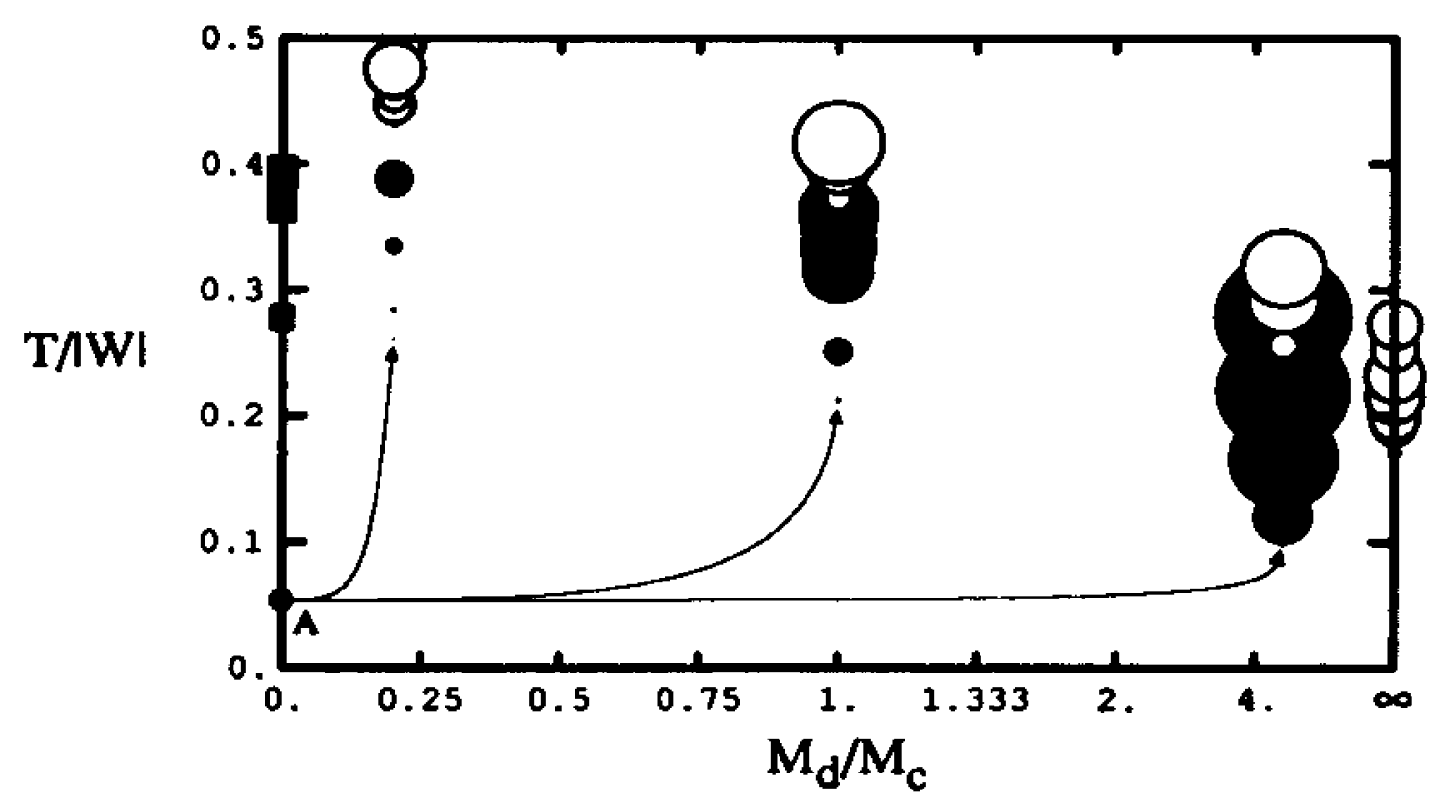

Figure 6.2: Possible evolutionary paths of protostellar disk/central object systems. Each path begins at point $A$, that is, at a time when a disk of insignificant mass has begun to form around the central protostar; $T /|W|$ is initially low because the material falling onto the disk is being heated through an accretion shock. Each path terminates when, upon cooling, the disk encounters a dynamically unstable configuration.

vs. $M_{d} / M_{c}$ diagram begins in a region of disk stability, then traverses the diagram towards the demarcation line which separates stable from unstable disks. To illustrate this general idea, three possible evolutionary paths have been illustrated in Figure 6.2; each path terminates when a dynamically unstable configuration is encountered. Consider the following evolutionary scenarios:

A.) If, as infalling material builds the disk up to an appreciable mass, the central object cools more rapidly than the disk surrounding it, the systern may evolve to a point where axisymmetric excretion occurs and material is transferred from the central object to the disk (Chapter 4). As material from the evaporating central object becomes incorporated into the disk (increasing the mass 
ratio $M_{d} / M_{c}$ ), the system will move rapidly to the right in Figure 6.2 and a dynamical, nonaxisymmetric instability will be encountered while the disk is still fairly hot. As we argued in $\$ 4.4$, however, the embedded central object is unlikely to cool significantly faster than the much more extended disk. This scenario, therefore, is unlikely to play a significant role in the determination of the resulting mass ratio of multiple stellar systems.

B.) If the disk within a protostellar system cools very rapidly, $T /|W|$ will increase rapidly, placing the system into a region where the $m=2$ mode dominates the subsequent evolution of the disk. If such were the case, the resulting system would, at most, become a trinary. A greater number of stellar companions could result from more severe cooling, placing the system at such a point that even higher order modes dominate. Such rapid cooling, however, would tend to rapidly change the internal dynamics of the disk, making the outcome of such an evolution impossible to predict using the results of this study as a guide.

C.) If the disk cools more gradually, evolving to a critical instability point without significantly disrupting the structure of the system, the initially axisymmetric disk will, regardless of the system's final mass ratio, first encounter an $m=1$ instability. This $m=1$ instability will be of one particular form. For all system mass ratios, the instability that will be encountered first resembles the one identified by Adams et al. (1989). A relatively tightly wound, one-armed spiral perturbation will develop in the disk and, in response, the central object will develop a spiraling orbital motion about the center of mass of the system. Following evolutionary scenario $\mathrm{C}$, as the nonaxisymmetric instability grows to nonlinear amplitude, we have observed that a significant quantity of disk material coalesces to one side of the central object at a radial distance roughly equivalent to the location of the original pressure maximum. This strongly suggests that 
the formation of a binary companion is underway. While the disk undergoes this global structural change, the orbital motion of the central object may grow to such a magnitude that material is drawn away from the disk, formally decreasing the system mass ratio or, perhaps, disrupting the disk. The resulting mass ratio and eccentricity of the binary system that will be formed in this manner cannot be indicated from the simulations performed in this dissertation due to a lack of extended nonlinear analyses. The simulations do suggest, however, that the most probable outcome of such protostellar systems is the formation of a binary stellar system. Nonlinear evolutions of the marginally unstable systems will provide a greater understanding of the evolution of these protostellar systems as the $m=1$ global instability drives the disk to become a binary stellar companion to the original central object.

In conclusion, the stability analyses carried out in this dissertation, coupled with sorne basic theoretical arguments related to the formation and growth of disks in protostellar systems, indicate that disk fragmentation is consistent with binary formation. Our stability study lays the foundation for simulations that can be carried well into the nonlinear amplitude regime and that, hopefully, will reveal the orbital properties of these newly formed systems. The examination of the properties of the systems resulting from extended nonlinear simulations can then be correlated with the observed frequency of occurrence and orbital properties of main-sequence and pre-main-sequence binary systems, as outlined in the introduction of this dissertation. At that point, the framework for a coherent picture of binary star formation from interstellar molecular clouds will be in hand. 


\section{REFERENCES}

Abt, H. A. 1987, ApJ, 317, 353

. 1983, ARA\&A, 21, 343

Abt, H. A., \& Levy, S. G. 1976, ApJS, 30, 273

Adams, F. C., Lada, C. J., \& Shu, F. H. 1987, ApJ, 312, 788

Adams, F. C., Ruden, S. P., \& Shu, F. H. 1989, ApJ, 347, 959

Andalib, S. 1992, Ph.D thesis, Louisiana State University, Baton Rouge

Arfken, G. 1985 Mathematical Methods for Physicists, 3rd ed., (Orlando: Academic Press, Inc.)

Beckwith, S. V. W., Sargent, A. I., Chini, R. S., \& Gusten, R. 1990, AJ, 99, 924

Binney, J., \& Tremaine, S. 1987, Galactic Dynamics (Princeton: Princeton University Press)

Black, D. C., \& Bodenheimer, P. 1976, ApJ, 206, 138

Bodenheimer, P., \& Boss, A. P. 1981, MNRAS, 197, 477

Bodenheimer, P., Ruzmaikina, T., \& Mathieu, R. D. 1991, in Protostars and Planets III, ed. E. H. Levy, J. Lunine, \& M. S. Mathews (Tucson: Univ. of Arizona Press), 000

Bodenheimer, P., Tohline, J. E., \& Black, D. C. 1980, ApJ, 242, 209

Bodenheimer, P., Yorke, H. W., Różyczka, M, \& Tohline, J. E. 1990, ApJ, 355, 651

Boss, A. P. 1980, ApJ, 237, 866

1986, ApJS, 62, 519

1991, in Protostars and Planets III, ed. E. H. Levy, J. Lunine, \& M. S. Mathews (Tucson: Univ. of Arizona Press), 000

Boss, A. P., \& Haber, J. G. 1982, ApJ, 255, 240

Cassen, P., \& Moosman, A. 1981, Icarus, 48, 353 
Cassen, P., Shu, F. H., \& Terebey, S. 1985, in Protostars and Planets II, ed. D. C. Black, \& M. S. Matthews (Tucson: Univ. of Arizona Press), 448

Christodoulou, D. 1990, Private Communication

Christodoulou, D. M., \& Narayan, R. 1992, ApJ, 388, 000

Drazin, P. G., \& Reid, W. H. 1981, Hydrodynamic stability (Cambridge: Cambridge Univ. Press)

Duquennoy, A., \& Mayor, M. 1991, A\&A, 248, 485

Frank, J., \& Robertson, J. A. 1988, MNRAS, 232, 1

Goodman, J., \& Narayan, R. 1988, MNRAS, 231, 96

Hachisu, I. 1986, ApJS, 62, 461

Hachisu, I., Tohline, J. E., \& Eriguchi, Y. 1987, ApJ, 323, 592

—. 1988, ApJS, 66, 315

Kojima, Y. 1986, Prog. Theor. Phys., 75, 251

Kopal, Z. 1959, Close Binary Systems, (New York: JohnWiley \& Sons)

Larson, R. B. 1972, MNRAS, 157, 121

Laughlin and Bodenheimer 1991, paper presented at Origins of Solar Systems/Star Formation Workshop, Santa Cruz, CA.

Lizano, S., \& Shu, F. H. 1989, ApJ, 342, 834

Miyama, S. M., Hayashi, C., \& Narita, S. 1984, ApJ, 279, 621

Monaghan, J. J., \& Lattanzio, J. C. 1990, ApJ, 375, 177

Narayan, R. 1990, in Structure and Emission Properties of Accretion Disks, ed. C. Bertout, S. Collin, J-P. Lasota, \& J. Tran Thanh Van (Singapore: Fong \& Sons), 153

Norman, M. L., Wilson, J. R., \& Barton, R. T. 1980, ApJ, 239, 968

Papaloizou, J. C. B., \& Pringle, J. E. 1984, MNRAS, 208, 721

Papaloizou, J. C., \& Savonije, G. J. 1991, MNRAS, 248, 353 
Pringle, J. E. 1985, in Interacting Binary Stars, eds. J.E. Pringle \& R.A. Wade (New York: Cambridge Univ. Press), 1

Ruzmaikina, T. V. 1988, in IAU Colloquium No. 99: Biosstronomy - the next steps, ed. G. Marx (Dordrecht: Kluwer), 41

Safranov, V. S., \& Ruzmaikina, T. V. 1978, in Protostars and Planets II, ed. D. C. Black, \& M. S. Matthews (Tucson: Univ. of Arizona Press), 959

Shu, F. H., Adams, F. C., \& Lizano, S. 1987, ARA\&A, 25, 23

Shu, F. H., Tremaine, S., Adams, F. C., \& Ruden, S. P. 1990, ApJ, 358, 495

Stemwedel, S., Yuan, C., and Cassen, P. 1990, ApJ, 351, 206

Strom, K. M., Strom, S. E., Edwards, S., Cabrit, S., \& Skrutskie, M. F. 1989, AJ, 97,1451

Terebey, S., Shu, F. H., \& Cassen, P. 1984, ApJ, 286, 529

Tohline, J. E. 1978, Ph.D thesis, University of California, Santa Cruz

- 1980, ApJ, 235, 866

- 1991, in Structure and Emission Properties of Accretion Disks, ed. C. Bertout, S. Collin-Souffrin, J. P. Lasota, \& J. Tran Thanh Van (Paris: Editions Frontieres), 131

Tohline, J. E., Bodemheimer, P. H., \& Christodoulou, D. M. 1987, ApJ, 322, 787

Tohline, J. E., \& Hachisu, I. 1990, ApJ, 361, 394; paper TH90

Williams, H. A. 1988, Ph.D thesis, Louisiana State University, Baton Rouge

Williams, H. A., \& Tohline, J. E. 1987, ApJ, 315, 594

Wilson, R. E. 1981, ApJ, 251, 246

van Albada, G. D. 1985, A\&A, 142, 491

van Albada, G. D., van Leer, B., \& Roberts, W. W. Jr. 1982, A\&A, 108, 76

van Leer, B. 1976, J. Comp. Phys., 23, 276 


\section{APPENDIX A \\ DETAILS CONCERNING THE HYDRODYNAMIC CODE}

\section{A.1 Introduction}

As stated in Chapter 2, the hydrodynamic code used in this stability survey solves a set of fluid equations over discrete intervals in time. In this appendix, we give the details of the finite-difference approximation to the fluid equations, discussing the specific method used to integrate these coupled equations forward in time. The equations involved include the continuity equation,

$$
\frac{d \rho}{d t}+\rho \nabla \cdot \mathrm{v}=0
$$

the equation of motion,

$$
\rho \frac{d \mathbf{v}}{d t}=-\nabla P-\rho \nabla \Phi,
$$

Poisson's equation,

$$
\nabla^{2} \Phi_{d}=4 \pi G \rho
$$

and an equation of state, given simply as

$$
P=K \rho^{\gamma}, \gamma \equiv 1+\frac{1}{n} .
$$

The equation of state is the most straightforward to use. A subroutine has been written which, when given an array of density values, returns an array of pressure values. This routine is a very simple one, and merits no more discussion. It does not play a part in determining the declared "order" of the hydrocode. Likewise, the Poisson equation does not determine the declared order of the code. Tohline (1978) discusses the method of solving the Poisson equation for the potential in 
detail. Section A.2 concerns the handling of source terms while §A.3 discusses the fluxing components of the principal fluid equations.

\section{A.2 Sourcing}

We begin the solution of the continuity equation and the equation of motion by casting them as nearly as possible into a conservative form, such as

$$
\frac{\partial x}{\partial t}+\nabla \cdot(x \mathbf{v})=0
$$

In such a form, the divergence term is referred to as the "fluxing" or "advection" term because this term indicates a transfer of the quantity $x$ over some displacement with a velocity $v$. In the case of the equation of continuity, the conservative form is

$$
\frac{\partial \rho}{\partial t}+\nabla \cdot(\rho \mathbf{v})=0
$$

and the divergence term, $\nabla \cdot(\rho \mathrm{v})$, indicates a flow of mass from one position to another. Likewise, the equation of motion can be cast into nearly-conservative form as

$$
\frac{\partial \mathbf{M}}{\partial t}+\nabla \cdot(\mathbf{M v})=-\nabla P-\rho \nabla \Phi,
$$

where we have defined the momentum density to be $M \equiv \rho v$. In this case, we are fluxing momentum from one region to another, but with an additional source of momentum resulting from the gradients in the pressure and gravitational potential. Rewriting the vector equation (A.7) as three component equations in cylindrical coordinates, we have

$$
\begin{aligned}
& \frac{\partial S}{\partial t}+\nabla \cdot(S v)=-\frac{\partial P}{\partial r}-\rho \frac{\partial \phi}{\partial r}+\frac{A^{2}}{\rho r^{3}}, \\
& \frac{\partial T}{\partial t}+\nabla \cdot(T v)=-\frac{\partial P}{\partial z}-\rho \frac{\partial \phi}{\partial z} \\
& \frac{\partial A}{\partial t}+\nabla \cdot(A v)=-\frac{\partial P}{\partial \theta}-\rho \frac{\partial \phi}{\partial \theta}
\end{aligned}
$$


where we have defined components of the momentum density, $S=M_{r}=\rho v_{r}$, $T=M_{z}=\rho v_{x}$, and $A=r M_{0}=\rho r v_{0}$. Since all four equations $(A .6, A .8 \mathrm{a}-\mathrm{c})$ present the same basic form, it is convenient to discuss one generic methodology for integrating them forward in time.

The source terms in the nearly-conservative fluid equations consist predominantly of simple, one-dimensional gradients in the pressure and gravitational potential. These gradients are approximated to third order by a simple Taylor series expansion. Assuming uniform spacing $\delta r$ between grid zones, the radial gradient in the pressure at the center of a grid zone $j$ is related to neighboring values of the pressure by

$$
\left(\frac{\partial P}{\partial r}\right)_{j}=\frac{P_{j+1}-P_{j-1}}{2 \delta r}+O\left(\delta r^{3}\right) .
$$

Thus, when a quantity such as the radial momentum density $S$ is updated via the source terms, the new momentum density, $S_{t+b t}$ is related to the current value $S_{t}$ through a very simple manipulation of other known quantities. The difficulty in constructing a second-order-accurate hydrodynamic code lies in proper handling of the fluxing components of the equations.

\section{A.3 van Leer Monotonic Interpolation}

The divergence (flux) terms in our equations can be rewritten as a summation over the faces of a grid zone as

$$
\nabla \cdot(X v)=\frac{1}{V} \sum_{i=1}^{6}(X v)_{i} A_{i}
$$

where $V$ represents the volume of the particular grid cell being examined, $A_{i}$ is the area of a particular face, and $(X v)_{i}$ is the product of the physical quantity $X$ with it's corresponding velocity $v$ at face $i$. The order of the fluxing scheme is 
derived from the method used to determine the value of the quantity $X$ to be used in the product with the velocity. (The velocities are, in general, straightforward to calculate.) For simplicity, we will consider only one-dimensional (radial) motion. In a first-order, donor cell scheme, if the velocity at the face of two adjoining cells is found to be outward from zone $j$ to $j+1$, the value of $X$ used is simply the value known at zone $\mathrm{j}$. If the velocity is inward from zone $j+1$ to zone $j$, the value used is $\boldsymbol{X}_{j+1}$. This methodology is simple in concept, simple to program, and results in first-order errors. It is this fluxing scheme that was used in the TH90 work.

A second-order fluxing scheme was developed by van Leer (1976). In this method, if the velocity is outward from zone $j$ to $j+1$, the value of $X$ used at the face is an interpolation from $X_{j}$ to the face of the grid zone. Likewise, for an inward velocity, the interpolation is from $X_{j+1}$ to the face of the grid zone. The interpolation is simple:

$$
X_{j+1 / 2}=X_{j}+\frac{1}{2} \delta r\left(\frac{\partial X}{\partial r}\right)_{j}
$$

for outward velocities and

$$
X_{j+1 / 2}=X_{j+1}-\frac{1}{2} \delta r\left(\frac{\partial X}{\partial r}\right)_{j+1}
$$

for inward velocities. The difficulty at this point comes from attempting to derive a second-order-accurate determination of the slopes $(\partial X / \partial r)$. One may be tempted to use a Taylor expansion to derive a second-order accurate slope, as was done in the handling of the sourcing terms. This does result in a second-order-accurate slope, but in practice the technique proves to be numerically unstable. van Leer (1976) proposed that the second-order methodology traditionally used could be viewed as a geometric mean of two first-order slopes derived from Taylor expansions, resulting in a second-order slope. In place of a geometric mean, one could use the harmonic 
mean of two first-order slopes:

$$
\frac{1}{(\partial X / \partial r)_{j+1 / 2}}=\frac{1}{2}\left(\frac{1}{(\partial X / \partial r)_{j}}+\frac{1}{(\partial X / \partial r)_{r}}\right)
$$

where the subscript $f$ indicates a forward-differenced, first-order slope

$$
\left(\frac{\partial X}{\partial r}\right)_{f}=\frac{X_{j+1}-X_{j}}{\delta r}
$$

and the subscript $r$ indicates a rearward-differenced, first-order slope

$$
\left(\frac{\partial X}{\partial r}\right)_{r}=\frac{X_{j}-X_{j-1}}{\delta r}
$$

The only point left to consider is the enforcement of monotonicity in the slope. If the forward and rearward first-order slopes differ in sign, the second-order slope is forced to zero. It is worth noting that the van Leer technique with a slope of zero is identical to the donor-cell technique.

The van Leer monotonic fluxing technique is, like the donor-cell technique, relatively straightforward to program. The penalty lies in the computational requirements of the scheme. For each time-step in an evolution, two first-order slopes must be calculated (none for the donor cell technique, of course), and then manipulated into a second-order slope for every grid zone. The end result is a fluxing scheme that is second-order accurate in spatial extent.

To obtain second-order accuracy in time, it is necessary to employ velocity values in the fluxing components that are centered at time $t+\delta t / 2$ (van Albada et. al 1982). In practice, this can be achieved by applying the source terms for a half time-step and saving the resulting quantities, then fluxing for half of a time-step to obtain velocities at $t+\delta t / 2$. Fluxing is then performed on the saved quantities over a full time-step, followed by an additional half time-step of sourcing. Again, programming this technique is straightforward. The penalty lies in execution time 
because two sourcing and two fluxing steps are performed for every single time-step. The results, however, of a fully second-order hydrodynamic code prove to be well worth the additional computational expense. 


\section{APPENDIX B \\ DETERMINATION OF $y_{1}$ AND $y_{2}$}

This Mathematica notebook is an example of how the data resulting from a hydrodynamic evolution is analyzed to determine the two parameters y1 and y2 (see equations 2.11 and 2.12) that identify the growth rate and the pattern speed of developing eigenmodes. The file that data is obtained from is a Fortran-formatted file containing a list of times in the evolution and the Fourier amplitudes and phases computed at each point in time. Once the location of the file is specified, one simply executes the notebook and Mathematica does the rest. Similar notebooks have been written to obtain eigenfunction plots, and to illustrate the motion of the central object in evolutions which allow for such motion.

Define the file to be used

1110 = "/Localveera/john/Mathomat ica . jw/modela/ oven_modes/mase_ratio_0.2/tw447/tw447.ma11";

\section{Definition of ApJPlot}

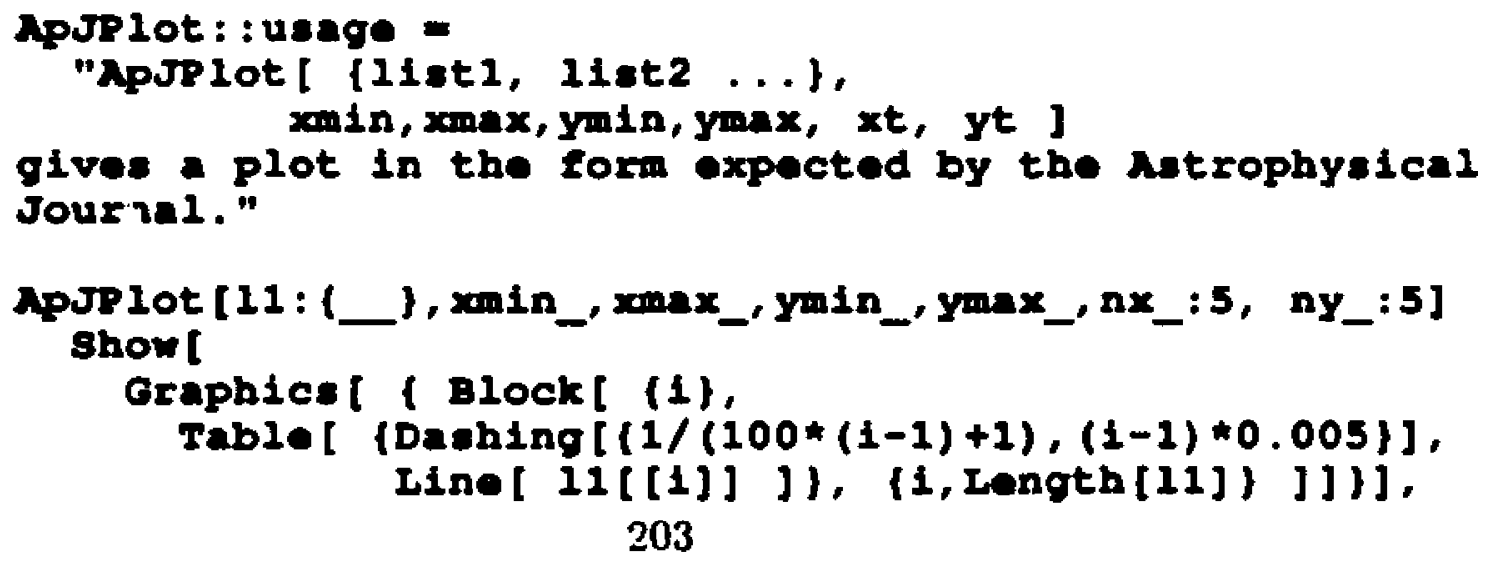


Graphice [Line $[\{(x \ln 1 n, y m i n),(x m n x, y m i n]$,

$\{\operatorname{man} x, y \max \},\{\operatorname{xin} i n, y \sin x\},(x \sin , \operatorname{ymin}\})] 1$,

Graphice [ ( Block [ (1),

Table I Inel I

$\{\operatorname{con} 1 n+(\operatorname{xmn} x-\operatorname{xan} 1 n) *(1-1) / n x, y \sin 1 n\}$,

$(\operatorname{xon} 1 n+(\operatorname{xannx}-x \sin 1 n) *(1-1) / n x$,

$y \min +(y \max -y \min ) / 50\}\}],\{1, n x\}]\}\}]$,

Graphice [ ( Block [ (1).

Table [ Ine [ I

$(x \min +(\operatorname{man} x-x \operatorname{con} i n) *(1-1) / n x, y \max )$,

$(\operatorname{xmin}+(\sin x x-\operatorname{xanin}) *(1-1) / n x$, $y \max -(y \max -y \min ) / 50)\}],(1, n x)]])]$,

Graphice [ I Block [ $\{1\}$,

Table I Ine [ I

(xmin, ymin+(ymax-ymin)*(1-1)/ny\},

$(\operatorname{xon} 1 n+(\sin x-\operatorname{xin} i n) / 50$,

$y \min +(y \max -y \min ) *(1-1) / n y\})],\{1, n y\}]]\}]$,

Graphice [ ( Block ( $(1)$,

Tablet Iine [t

$(\operatorname{xan} x, \operatorname{ymax}-(\operatorname{ymax}-\operatorname{ym} 1 n) *(1-1) / n y\}$,

$(\operatorname{xan} x-(\sin x-x \operatorname{con} i n) / 50$,

$\operatorname{ymax}-(\operatorname{ymax}-\operatorname{ymin}) *(1-1) / n y)\}],(1, n y\}]]\}]$,

Graphice $[$ Block $[\{x\}$,

Table $[T e x t[N[x, 2],\{x, y \min -(y \max -y \min ) / 20\}]$,

$\{x, \operatorname{xin} i n, \operatorname{xin} x,(\operatorname{xmax}-x \operatorname{con} i n) / n x)]]\}]$,

Graphice [ ( Block [ (y),

Tab] $[$ Text $[N[y, 2],(\operatorname{xan} i n-(\operatorname{xmax}-\operatorname{xan} 1 n) / 50, y\}$,

Graphice [

$\{1,0\}],\{y, y \min , \operatorname{ymax},(\operatorname{ymax}-\operatorname{ymin}) / n y\}]]\}]$,

Table [Text [" (t/t_rot)" , $(\operatorname{xanin}+((\operatorname{xanax}-\operatorname{xan} i n) / 2)$,

Graphicet $\operatorname{yan} i n-(\sin x-\operatorname{ym} 1 n) / 7] J]]$,

Table [Text ["In (D)", $(\operatorname{xan} n-(\operatorname{xan} x-\operatorname{xan} i n) / 8$, ymax- $(y \max -y \min ) / 2),(1,0)] 11$,

PlotRange $\rightarrow 1$

$(x \min -(\max x-\operatorname{xan} 1 n) / 4, \operatorname{xin} x+(\operatorname{xmax} x \operatorname{xm} 1 n) / 8)$,

$(y \min -(y \max -y \min ) / 4, y \max +(y \max -y m i n) / 8)\}]$

ApJPlot [ $\{1$ ist 1, list $2 \ldots\}$,

xmin, xmax, ymin,ymax, $x t, y t$ ] gives a plot in thel

form expected by the Astrophysical Journal.

\section{Setup}

Read in the data and set up some definitions 


$$
\begin{aligned}
& \text { rm4amp }[n]]:=\operatorname{amp} 2[1,2, n] ; \\
& \text { r2m4amp }[n]]:=\operatorname{amp} 2[2,2, n] ; \\
& \text { r3m4amp }[n]]:=\operatorname{amp} 2[3,2, n] ; \\
& \text { rim6amp }[n]]:=\operatorname{amp} 2[1,3, n] ; \\
& \text { r2m6amp }[n]]:=\operatorname{amp} 2[2,3, n] ; \\
& \text { r3m6amp }[n]]:=\operatorname{amp} 2[3,3, n] ;
\end{aligned}
$$

Set up tables for $m=1, m=2, m=3$ (or $m=2, m=4, m=6$ for even modes)

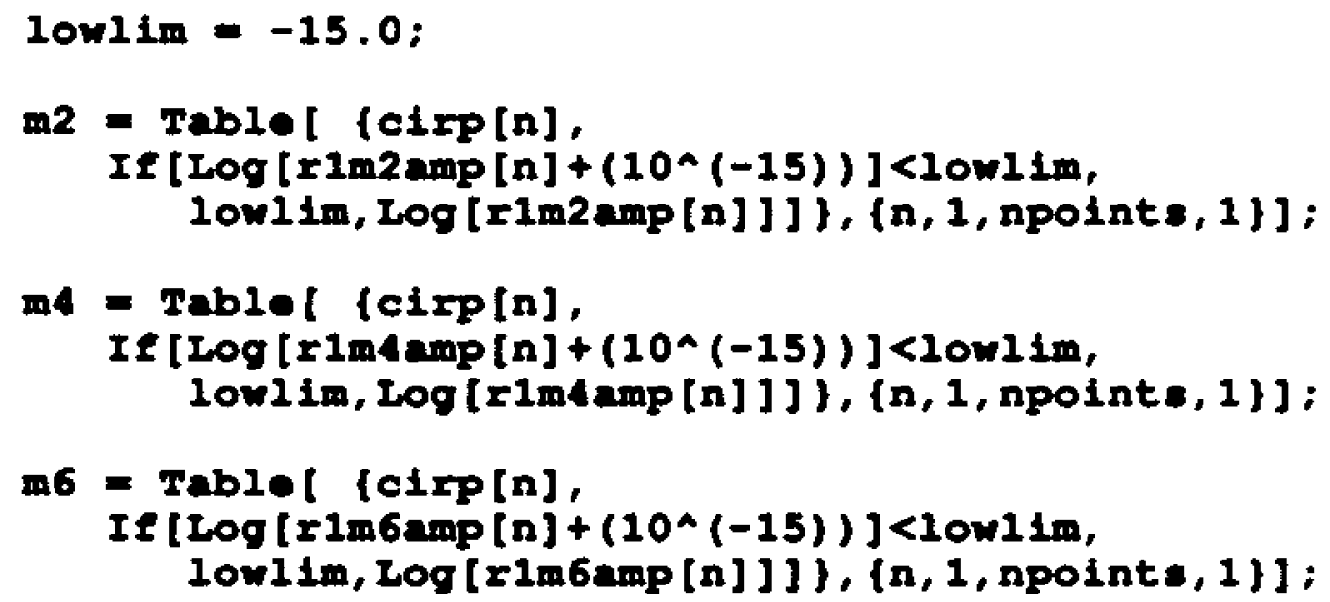

Fit the data (note that we only fit a certain region).

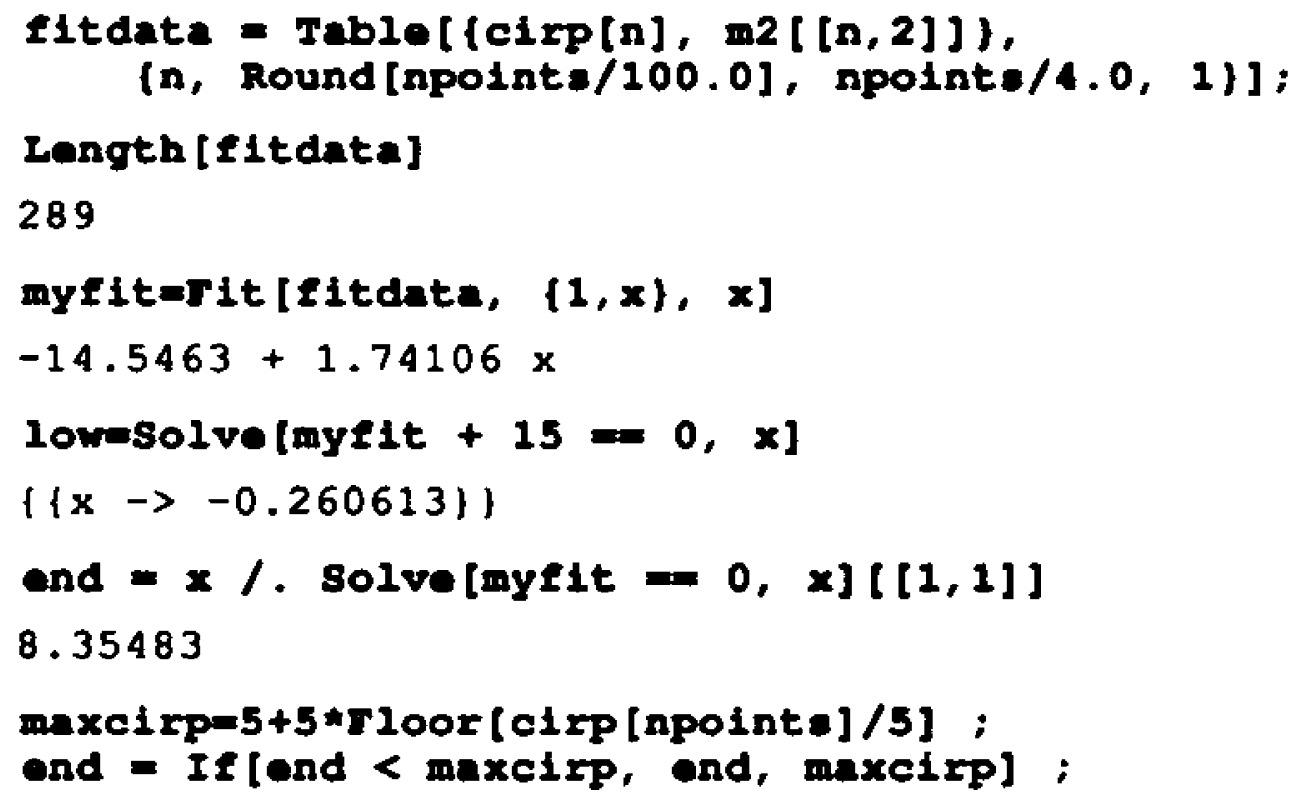




\section{Plot out the final result}

Dlot the amplitude versus time behavior first:

ApJP lot $[(m 2, m 4, m 6, \operatorname{Tab} 1 \in[\{x, m y \in i t\},\{x, 0$, and, and $\}]\}$, $0, \operatorname{maxc}[\mathrm{p}, 10 \mathrm{w} 1 \mathrm{~m}, 0]$

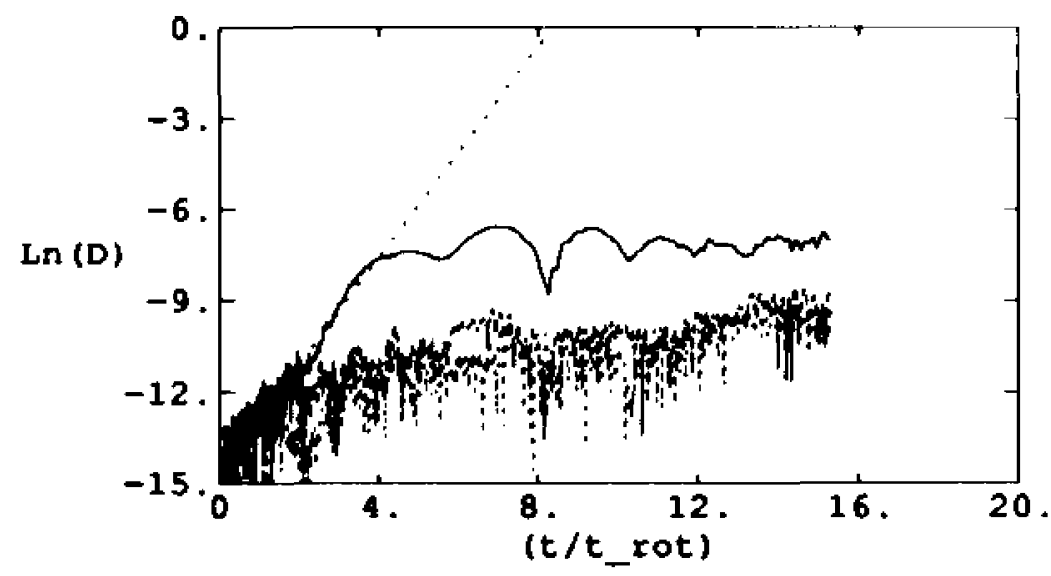

-Graphics-

Now plot the phase versus time behavior:

ApJP lot [ (m2phase), 0 , maxcirp, 0, 1]

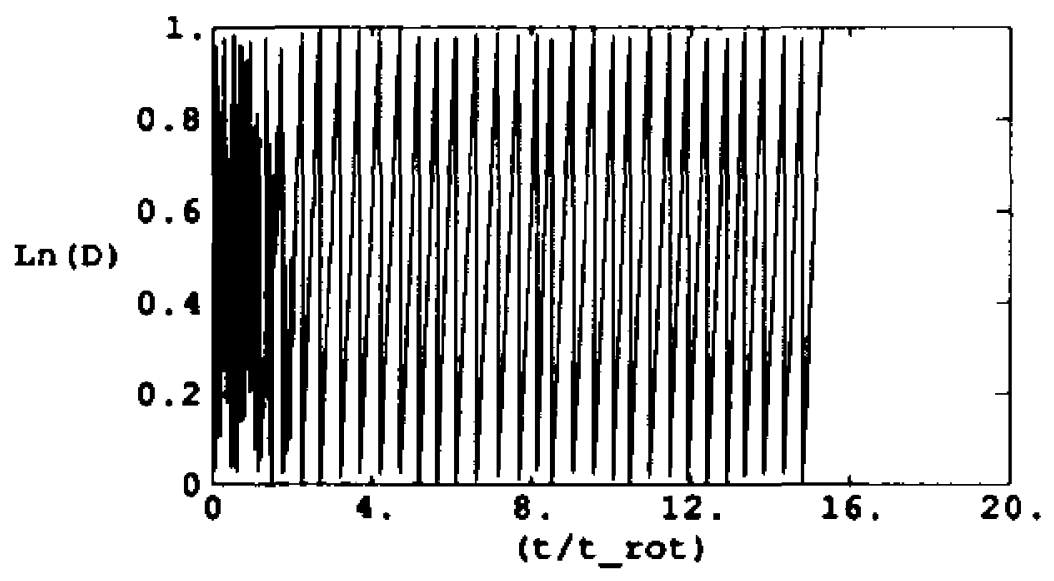

-Graphics- 
Phase factor (from measurements on graph)

$$
\begin{aligned}
& (15.0) /(14.818-7.698)-2.0 \\
& 0.106742
\end{aligned}
$$

Growth rate, $y_{-} 2(m=1)$ (from derivative of fit)

$$
\begin{aligned}
& N[D[\text { myfit }, x] /(2 P 1)] \\
& 0.277098
\end{aligned}
$$




\section{APPENDIX C \\ CONVERSION TO PHYSICAL UNITS}

The hydrodynamic code used in this survey performs all calculations in dimensionless units. By scaling the gravitational constant, $G$, the mass of the disk, $M_{d}$, and the polytropic constant, $K$, to unity, the results of any calculation can be scaled to any size system desired. The conversion from "program units" to "physical units" is performed simply by a choice of disk mass $M$ and polytropic constant $K$, followed by a multiplication by appropriate combinations of the constants $G$, $K$, and $M$. This appendix presents the general form of the conversion, along with a few examples (see Williams and Tohline 1987 for a similar presentation for centrally condensed polytropes).

From the polytropic relation, $P=K^{*} \rho^{\gamma}$, where $\gamma=1+\frac{1}{n}$, we see that $K$ involves units of mass, distance, and time as $m^{-1 / n} r^{2+3 / n} t^{-2}$. Likewise, the gravitational constant $G$ has units $r^{3} m^{-1} t^{-2}$. A multiplicative combination of $G, K$, and the desired mass $M$ to obtain a quantity $x$ with units $m^{\lambda} r^{\sigma} t^{\ell}$ can be written

$$
x_{\text {real }}=x_{\text {prog }} G^{a} K^{-b} M^{c} .
$$

The exponents $a, b$, and $c$ are related to the exponents $\lambda, \sigma$, and $\epsilon$ through the expressions:

$$
\begin{aligned}
a & =\frac{1}{n-3}\left[\left(\frac{3}{2}+n\right) \epsilon+n \sigma\right], \\
b & =\frac{-n}{n-3}\left[\frac{3}{2} \epsilon+\sigma\right], \\
c & =\lambda+\frac{1}{n-3}[\sigma(n-1)+\epsilon n] .
\end{aligned}
$$

To avoid the somewhat arbitrary choice of $K$ used in the conversion of units, it is conceptually easier to choose the radial extent of the system and derive an 
appropriate value of $K$. We see that a variable with units of $r$ has $\lambda=0, \sigma=1$, and $\epsilon=0$. Using the relations in Equations C.2a-C.2c, we obtain $a=n /(n-3)$, $b=-n /(n-3)$, and $c=(n-1) /(n-3)$. The conversion of radial position is then performed as

$$
R_{\text {real }}=R_{\text {prog }}\left[G^{n} K^{-n} M^{n-1}\right]^{\frac{1}{n-1}}
$$

Using this relation, we can specify a radial extent $R_{\text {real }}$ and solve for the appropriate $\boldsymbol{K}:$

$$
K=G M^{1-\frac{1}{n}}\left(\frac{R_{\text {real }}}{R_{\text {prog }}}\right)^{\frac{2}{n-1}} .
$$

By substituting Equation $C .4$ into Equation $C .1$ and proceeding in a straightforward manner, the density $\rho(\lambda=1, \sigma=-3, \epsilon=0)$ scales as

$$
\rho_{\text {real }}=\rho_{\text {prog }}\left[M\left(\frac{R_{\text {prog }}}{R_{\text {real }}}\right)^{3}\right],
$$

whereas the rotational frequency $\Omega(\lambda=0, \sigma=0, \epsilon=-1)$ scales as

$$
\Omega_{\text {reat }}=\Omega_{\text {prog }}\left[G\left(\frac{R_{\text {real }}}{R_{\text {prog }}}\right)^{-3} M\right]^{1 / 2}
$$

Choosing a disk mass $M=1 M_{\odot}$, extending outward for $R_{\text {real }}=100 \mathrm{~A} . U$, and using data obtained from \$5.4.2, model \#2 (an excellent example of the spiral instability), we find that the time for one rotation is

$$
t_{\text {rot }}=\frac{2 \pi}{\Omega_{\text {real }}}=113 y r s
$$

We see that this model, with $y_{2}(1)=0.179$, has an e-folding time of slightly more than 100 years, and that the evolution of the model covered somewhat more than 1000 years. In addition, the maximum density in the initial model is found to be

$$
\rho_{\max }=1.3 \times 10^{-12} \mathrm{~g} / \mathrm{cm}^{3}
$$


The temperature of the gas cloud can be calculated through the ideal gas equation coupled with the polytropic equation of state. These two equations lead us to an expression for the temperature of the gas:

$$
T=\frac{m_{h} K}{k} \rho^{1 / n},
$$

where $k$ is Boltzmann's constant. Thus, the maximum temperature in the disk is found via the maximum density, and is

$$
T_{\max } \approx 1000^{\circ} \mathrm{K}^{*}
$$

Clearly, with $k T \approx 0.1 e v$, this is a disk composed of non-ionized, molecular hydrogen. 


\section{APPENDIX D}

\section{A DIRECT COMPARISON WITH LINEAR THEORY}

While comparisons of the results of the second-order hydrocode against the results of the older first-order hydrocode give us confidence in the overall accuracy of the code, a direct comparison of the hydrocode against the results of linear theory is required. Linear theory studies of zero-mass disks have been performed (Papaloizou \& Pringle 1984, Kojima 1986, and Frank \& Robertson 1988, for example), and we have referred to these results in the main body of this dissertation. In order to compare our hydrocode against the $M_{d} / M_{c}=0$ linear results, the gravitational potential of the disk must be completely removed from our computations. As such, we delete the potential solver from the hydrocode and explicitly set the disk potential to zero. With this change in the code in mind, we see the comparison of $M_{d} / M_{c}=\infty$ systems performed in Chapter 2 between the first-order and second-order hydrocodes is still very relevant, as this provides a conclusive test of the portion of the code that solves self-consistently for the disk potential.

Instead of using the HSCF technique, in this case initial models are created through an explicit equation governing the axisymmetric density distribution (Papaloizou \& Pringle 1984). We generate 6 models, each having a rotation law governed by $q=2$, and a polytropic index $n=3$. In this manner, the models we generate for the comparison correspond precisely to the systems examined by Kojima (1986). In the Kojima (1986) linear analysis, the systems are parameterized by $r_{-} / r_{0}$. The data describing the models generated for this comparison are tabulated in Table D.1.

Each model is evolved as described in Chapter 2, using a radial/vertical/azimuthal resolution of $64 / 32 / 64$, except for model \#6 $\left(r_{-} / r_{0}=0.85\right)$, for which it was 
necessary to use a resolution of $128 / 32 / 64$ in order to adequately resolve the structure of the disk. Key results from the evolution of these models are tabulated in Table D.2. The growth rate parameter $y_{2}(m)$ and the pattern speed parameter $y_{1}(m)$ for $m=1$ and $m=2$ are illustrated in the upper/lower panels of Figure D.1. Because these evolutionary sequences have been computed only for comparison purposes and are not directly related to the focus of this dissertation, only one representative example of the radial eigenfunction $(\phi-r$ plot) is illustrated, along with the corresponding $D_{m}-r$ plot (Figure D.2).

Examining Figure D.1, it is clear that the results of the second-order code compare quite well with the results of linear theory. Our mensured $y_{1}(m)$ parameter in particular corresponds with the Kojima (1986) results to a very high degree of accuracy. There are noticeable discrepancies in the growth rates of the models evolved; unstable modes examined with the second-order hydrocode exhibit growth rates that are consistently less than what is predicted by linear theory. In addition, towards lower values of $r_{-} / r_{0}$, the hydrocode does not reveal any of the cyclic behavior in the value of $y_{2}(m)$ that was observed by Kojima (1986) and by Frank and Robertson (1988). We suggest, although we cannot prove at this point in time, that these differences are due to nonlinear effects. The nonlinear hydrocode allows interactions between various modes, whereas linear treatments of these systems completely decouple all modes, stifling interactions. Indeed, when simulations were performed without imposing the $\pi$-symmetry constraint, the $y_{2}(2)$ parameter that was measured from the simulations completely disagreed with the linear predictions. [The $y_{2}(2)$ parameter itemized in Table D.2 and displayed in Figure D.1 reflects results from $\pi$-symmetry evolutions.] In these unconstrained simulations, the $m=2$ (and higher order) modes grew to an appreciable amplitude and appeared to be locked in phase with the $m=1$ mode. (Analogously, in $\pi$-symmetry simulations, 
$m=4$ and higher order modes developed in phase with the $m=2$ mode.) $T$ his leads us to conclude that modal interactions are occurring in the hydrocode simulations, effectively transferring power away from the dominant $m=1(m=2)$ mode to the higher order modes. Hence, the growth rate of the $m=1(m=2)$ mode is reduced somewhat, while the higher order modes exhibit growth rates higher than what one would expect from the linear analyses.

In summary, the simulations of $M_{d} / M_{c}=0$ systems agree quite well with the results of linear theory. The $y_{1}(m)$ parameters presented throughout this dissertation, based upon this agreement, will require no correction when strictly linear analyses are performed upon comparable systems. Linear analyses of comparable systems will, however, result in $y_{2}(m)$ values higher than those presented here; we attribute the difference in growth rates to nonlinear mode couplings which cannot be determined through linear analyses. 
Table D.1

Initial Model Data for $M_{d} / M_{c}=0$

\begin{tabular}{ccccc}
\hline \hline Model \# & $r_{-} / r_{+}$ & $r_{0} / r_{+}$ & $r_{-} / r_{0}$ & $T /|W|$ \\
1 & 0.2 & 0.333 & 0.60 & 0.410 \\
2 & 0.3 & 0.461 & 0.65 & 0.452 \\
3 & 0.4 & 0.571 & 0.70 & 0.471 \\
4 & 0.5 & 0.666 & 0.75 & 0.483 \\
5 & 0.6 & 0.750 & 0.80 & 0.491 \\
6 & 0.7 & 0.823 & 0.85 & 0.495 \\
\hline
\end{tabular}




\section{Table D.2}

Eigenmode Character, $M_{d} / M_{c}=0$

\begin{tabular}{ccccccccc}
\hline & \multicolumn{9}{c}{$m=1$} & & \multicolumn{2}{c}{$m=2$} \\
\cline { 2 - 6 } \cline { 4 - 7 } Model & $y_{1}$ & $y_{2}$ & $\Omega_{p} / \Omega_{0}$ & $r_{c r} / r_{0}$ & $r_{c r} / r_{+}$ & $y_{1}$ & $y_{2}$ \\
1 & -0.32 & 0.111 & 0.68 & 1.21 & 0.40 & -0.53 & - \\
2 & -0.26 & 0.162 & 0.74 & 1.16 & 0.53 & -0.48 & 0.0373 \\
3 & -0.17 & 0.171 & 0.83 & 1.09 & 0.62 & -0.26 & 0.0802 \\
4 & -0.087 & 0.165 & 0.91 & 1.04 & 0.69 & -0.17 & 0.1742 \\
5 & -0.066 & 0.104 & 0.93 & 1.03 & 0.77 & -0.14 & 0.1814 \\
6 & -0.025 & 0.098 & 0.96 & 1.02 & 0.84 & -0.025 & 0.1483 \\
\hline
\end{tabular}



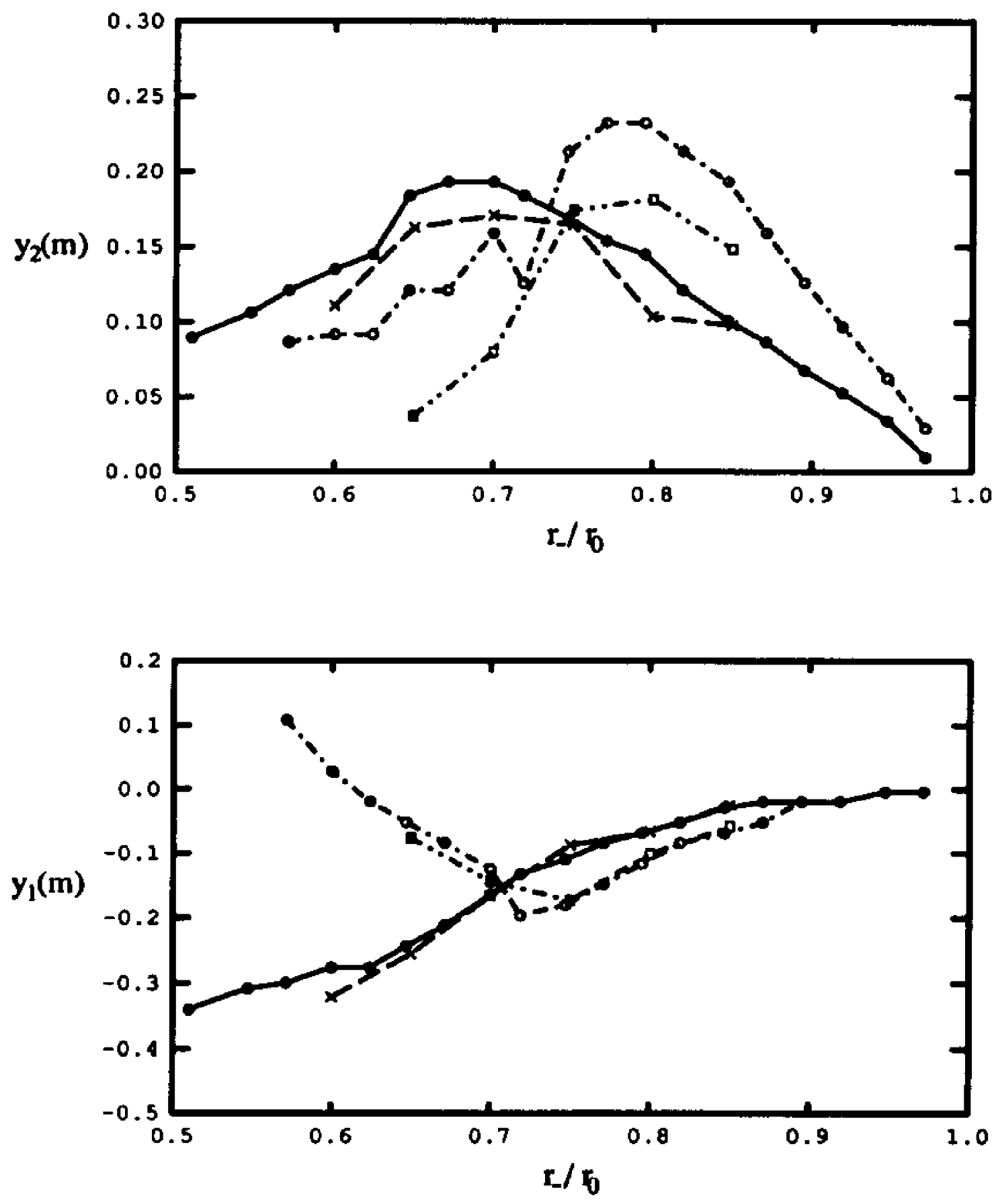

Figure D.1: $y_{2}(m)$ and $y_{1}(m)$ as functions of $r_{-} / r_{0}$ for all $M_{d} / M_{c}=0$ models; $m=1$ linear results (solid circles), $m=1$ hydrodynamic results (crosses), $m=2$ linear results (open circles), and $m=2$ hydrodynamic results (boxes). 

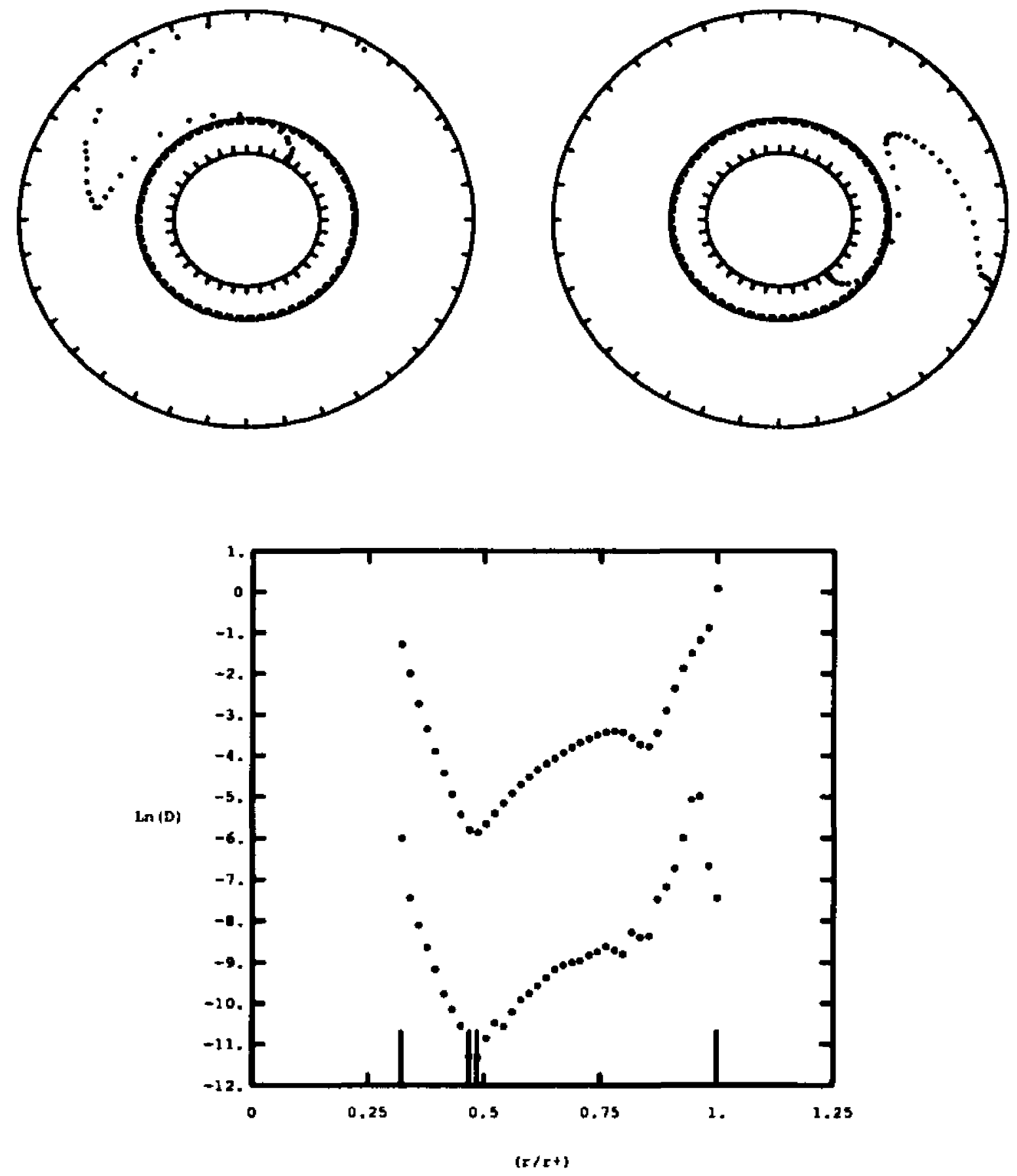

Figure D.2: The radial eigenfunction $\left(\phi_{1}-r\right)$ and amplitude as a function of radius $\left(D_{1}-r\right)$ of the $m=1$ Fourier mode illustrated as in Figure 2.3b for $M_{d} / M_{c}=0$, model \#2. Note the development of a leading $\pi / 2$ phase shift in the upper panels. 


\section{VITA}

John Woodward was born in Wurzburg, Germany in September 1964. Growing up in a military family, he has lived in many locations in the United States as well as West Germany. Introduced to the computer in 1976 by an older brother, he has yet to stop programming. Having a natural aptitude for mathematics and for the sciences, John received a bachelors degree in physics from the University of Texas in El Paso in 1986, married Laura Lee Kajp in 1987, and has continued his studies, applying his programming skills to simulations of the early stages of star formation. His wife and three children are understandably pleased that he is receiving his doctorate in physics in May 1992. 


\section{DOCTORAL EXAMINATION AND DISSERTATION REPORT}

Cendidnte: John William Woodward

mor Fields Physics

ritle of Diecertation:

The Stability of Thick, Self-Gravitating Disks in Protostellar Systems

Approned:
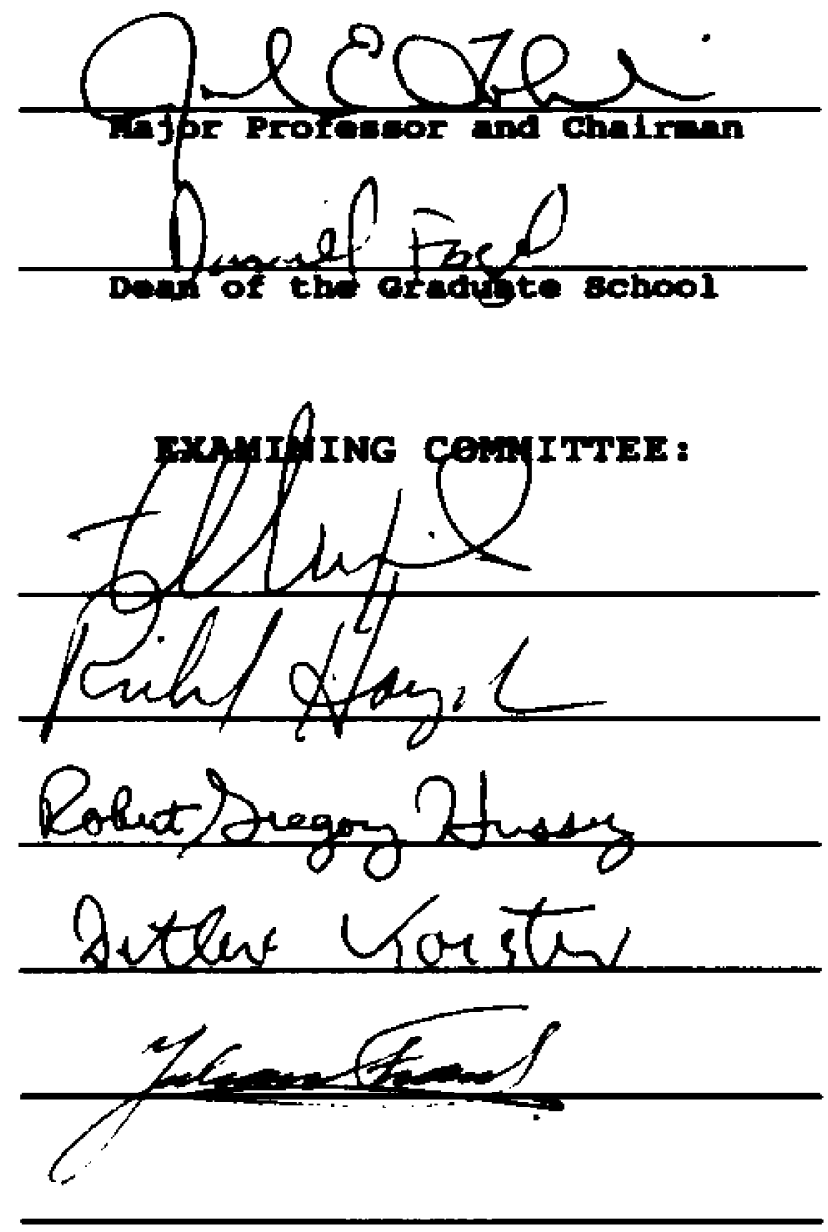

Date of Exerinations

March 20, 1992 